NASACONTRACTOR REPORT

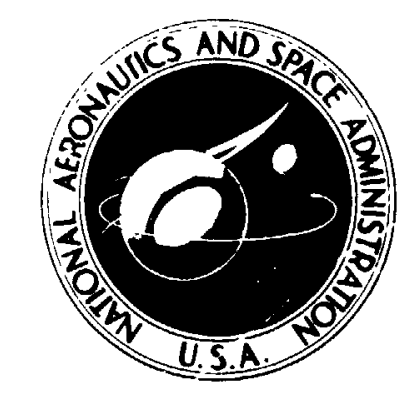

NASA CR-2788

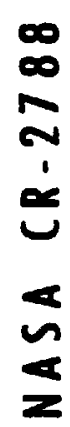

(AASA-6-2788) SIAEIIIIY AND CONTEOL OF

DANPI AING HIGH-EEFFCFEANCE AIFCEAFT EIDAI

Eeport (analytic Sciences Corf.) $206 \mathrm{FHC}$

A 10/AF 01 CSCL $01 \mathrm{~L}$

N77-21100

Unclas

H1/08 20784

\title{
STABILITY AND CONTROL OF MANEUVERING
}

\section{HIGH-PER.FORMANCE AIRCRAFT}

Robert F. Stengel and Paul W. Berry

Prepared by

THE ANALYTIC SCIENCES CORPORATION

Reading, Mass. 01867

for Langley Research Center

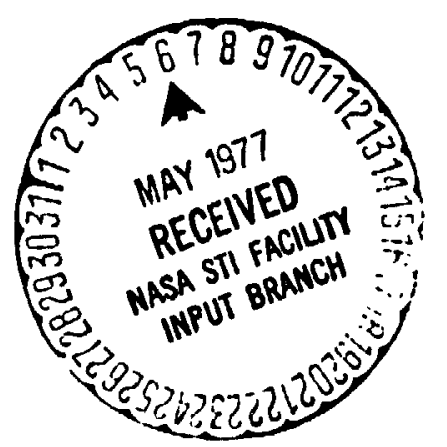

NATIONAL AERONAUTICS AND SPACE ADMINISTRATION - WASHINGTON, D.C. APRIL 1977 


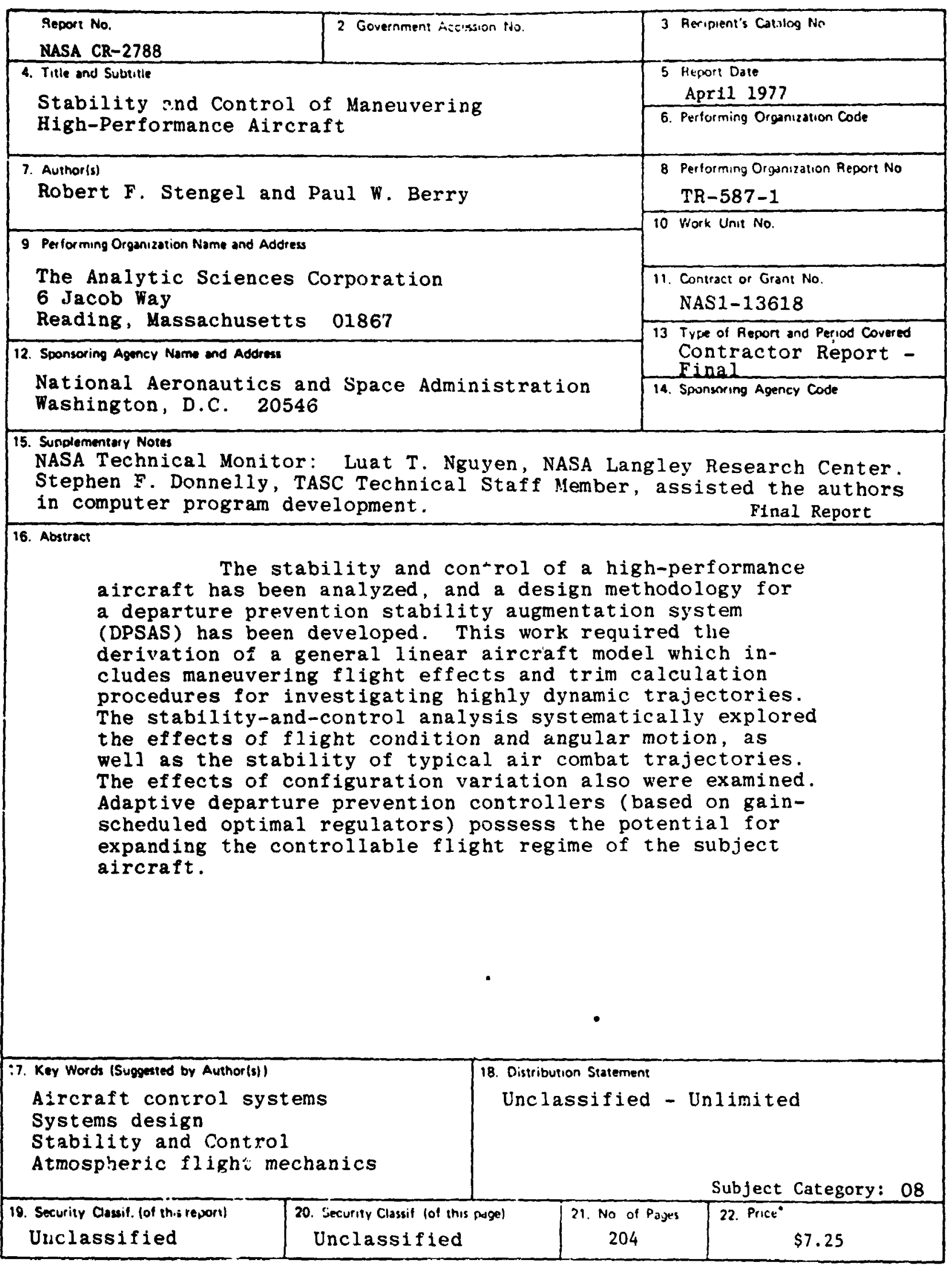

- For sale by the National Technical informalion Service Springlield Virginia 22161 
TABLE OF CONTENTS

List of Figures

Page

No.

List of Tabies

$\mathrm{v}$

List of Symbols

vii

ix

1. INTRODUCTION 1

1.1 Background 1

1.2 Purpose 4

1.3 Summary of Results 4

1.4 Organization of the Report 7

2. DYNAMIC CHARACTERISTICS OF HIGH-PERFORHANCE AIRCRAFT 8

2.1 Overview 8

2.2 Prior Studies of Aircraft at Extreme

2.2.1 Dynamics of the Aircraft 10

2.2.2 Aerodynamics 14

2.2.3 Control 15

2.3 Comparison of Results from Linear and
Nonlinear Simulations

2.3.1 Elevator Control Input 19

2.3.2 Aileron Control Input 20

2.3.3 Rudder Control Input 20

2.4 Effects of Angular Motion and Flight Condition on
Aircraft Stability

2.4.1 Altitude and Velocity Effects 27

2.4.2 Aerodynamic Angle Effects 30

2.4.3 Angular Rate Effects 38

2.5 Effects of Angular Motion and Flight Condition on
Aireraft Control

2.5.1 Velocity and Aerodynamic Angle Effects

2.5.2 Angular Rate Effects 49

2.6 Dynamic Variations During Extreme Maneuvering 51

2.6.1 Wind-Up Turn 52

2.6.2 Rolling Reversal 54

2.6.3 Effects of Proportional Tracking 57

2.7 Chapter Summary 59

3. EFFECTS OF CONFIGURATION VARIATIONS ON AIRCRAFT DYNAMICS 62

3.1 Overview 62

3.2 Variations Due to Longitudinel Stability Derivatives 62

3.3 Variations Due to Lateral-Directional

Stability Derivatives $\quad 68$ 
3.4 Variations Due to Mass and Inertia Effects $\quad 74$

3.5 Classification of Departures

3.6 Chapter Summary

4. PREVENTION OF DEPARTURE FROM CONTROLLED FLIGHT 86

4.1 Overview 86

4.2 The Linear-Optimal Regulator 89

$\begin{array}{lll}4.3 & \text { DPSAS Control Laws } & 94\end{array}$

4.3.1 Longitudinal Sweep 97

4.3.2 Lateral-Directional Sweep 103

4.4 Control-Law Adaptation for Varying Flight Conditions 112

4.4.1 Longitudinal Sweep 114

$\begin{array}{ll}\text { 4.4.2 Lateral-Directional Sweep } & 117\end{array}$

4.4.3 Additional Considerations 119

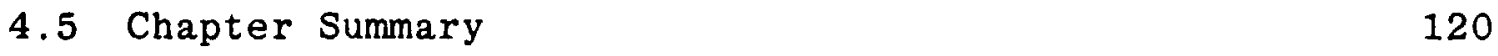

5. CONCLUSIONS AND RECOMMENDATIONS 122

5.1 Conclusions 122

$\begin{array}{ll}5.2 & \text { Recommendations } \\ & 124\end{array}$

APPENDIX A ANALYTICAL APPROACH TO AIRCRAFT DYNAMICS $A-1$

APPENDIX B AIRCRAFT AERODYNAMIC MODEJ, B

REFERENCES $\quad$ R-1 


\section{LIST OF FIGURES}

Figure

No.

2. 3-1

2. 3-2

2. 3-3

2. 3-4

$2.3-5$

2. 4-1

2. 4-2

2. 4-3

2. 4-4

2. 4-5

2. 4-6

2. 4-7

2. 4-8

2. 4-9

2. 4-10

2. 5-1

2. 6-1

3. 2-1

3. 2-2

3. 3-1

3. 4-1

2. 5-1

Small Amplitude Elevator Input

Page

No.

Small Amplitude Aileron Input

20

21

Large Amplj.tude Rudier Input-- Comparison of Initial Pesponse

24 E.jlved Response With Ad Hoc Reference Point for Linearization

Large Ampiitude Rudder Input-- Comparison of

Eiolved Response With Generalized Trim Reference

Point for Linearization

Altitude and Velocity Effects on Eigenvalues

28

Effects of Aerodynamic Angles on Aircraft Stability

31

Angle-of-Attack Effects on Aircraft Eigenvectors

33

Sideslip Effect on Aircraft Eigenvectors

35

Effects of Body Orientation on Aircraft Stability

36

Variations of Directional Aerodynamic Coefficients witin Angle of Attack

37

39

Yaw-Rate/Pitch-Rate Effects

40

Pitch-Rate Effects on Eigenvectors

42

Variations $\left(\alpha_{0}=15 \mathrm{deg}\right)$

43

45

Typical Step Response Forms

56

Eigenvectors of Rolling Reversal

Longitudinal Eigenvalue Variations with c.g. Location 66

Longitudinal Eigenvector Variations with

c.g. Location

$6 \epsilon$

Effects of $C_{I_{\beta}}, C_{n_{\beta}}$, and $C_{I_{p}}$ Variations on Lateral-

Directional Eigenvalues

Effects of Large Rolling Inertia on Aircraft

Stability

77

An Example of Lateral-Directional Eigenvalues for Negative Directional Stability 


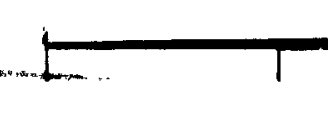

\section{LIST OF FIGURES (Continued)}

Figure

Page

No.

No.

3.5-2 An Unforced Departure Due to Negative $C_{n_{\beta}}$

3. 5-3

An Unforced Departure Duẹ to Negative Dutch Ro11 Damping

3.5-4 Eigenvalues and Eigenvectors for a Flight Condition with Large Sidesilp Angle

3.5-5 Aileron Input for Negative $C_{n_{B}}$

4.2-1 Longitudinal Response at the Central

\section{Flight Condition}

4.2-2 Directional Response at-the Central Flight Condition 95

4.2-3 Lateral Response at the Central Flight Condition 96

4.3-1 Pitch-Rate Effect on Directional Response 102

4.3-2 Exampies of Primary Gain Variation in LateralDirectional Sweep

4.3-3 Examples of Crossfeed Gain Variation in LateralDirectional Sweep

$4.3-4$

Roll-Rate Effect on Directional Response

109

4. $3-5$

Roll-Rate/Sideslip Effect on Longitudiral Response

110 


\section{LIST OF TABLES}

Table

Page

No.

No.

2.4-1 Dynamic Effects of Steady Angular Rate 39

2.5-1 Velocity Effects on Transfer Function Gain, $\mathrm{K}_{\mathrm{F}} \quad 46$

2.5-2 Aerodynamic Angle Effects on Transfer Function Gains $\begin{array}{ll}\left(V_{0}=94 \mathrm{~m} / \mathrm{s}\right) & 47\end{array}$

2.5-3 Effects of Angle of Attack on Transfer Function Zeros 48

2.5-4 Pole-Zero Comparison at $\alpha_{0}=15 \mathrm{deg}, B_{0}=10 \mathrm{deg} \quad 48$

2.5-5 Effects of Pitch Rate on Transfer Function Gains 50

2.5-6 Effects of Roll Rate on Transfer Function Gain, $K_{F} 50$

2.6-1 Wind-Up Turn Working Points 53

2.6-2 Wind-Up Turn Eigenvalues 53

2.6-3 Transfer Function Gain, $K_{I}$, Along the Wind-Up Turn 54

2.6-4 Rolling Reversal Working Points 55

2.6-5 Rolling Reversal Eigenvalues 55

2.6-6 Transfer Function Gain, $\mathrm{K}_{I}$, Along the Rolling Reversal57

2.6-7 Eigenvalue Changes due to Proportional Tracking -Symmetric Flight Conditions

2.6-8 Eigenvalue Changes Due to Proportional Tracking -Sideslip and Roll Effects

3.2-1 $\mathrm{C}_{\mathrm{Z} \alpha}$ and $\mathrm{C}_{\mathrm{m}_{\mathrm{q}}}$ Effects on Eigenvalues 63

3.2-2 Effects of $\mathrm{C}_{\mathrm{m}_{\mathrm{q}}}$ on Eigenvalues in Asymmetric Fight 64

3.2-3 c.8. Location Effects on Transfer Function Gain, $K_{F} 67$

3.2-4 Comparison of Zeros of $\Delta w / \Delta \delta_{h}$ at Three c.g. Locations 67

3.3-1 Effects of $C_{n_{B}}$ and $C_{1}$ Variations in the Presence $\quad 70$

3.3-2 Effects of $C_{n_{r}}$ Variations $\quad 71$

3.3-3 Effects of $C_{n_{B}}$ in the Presence of Steady Rolling 73 


\section{LIST OF TABLES (Continued)}

Table

Page

No.

No.

3.4-1 Effects of Aircraft Mass on Eigenvalue Location

3. 4-2 Effect of Rotational Inertia on Eigenvalues

4. 2-1 Effects of DPSAS at the Central Fight Condition

$4.2-2$ DPSAS Gain Matrix at the Central Flight Condition

4. 3-1 Closed-Loop Stability in the Longitudinal Sweep DPSAS Gains for the Longitudinal Sweep

$4.3-3$ Closed-Loop Stability in the LateralDirectional Sweep

4. 4-1

Gain Correlations for the Longitudinal Sweep

4. 4-2 Gain Correlations for the LateralDirectional Sweep 


\section{LIST OF SYMBOLS}

In general, matrices are represented by canital letters and vectors are underscored. The subscript on a vector usually indicates the frame in which the vector is expressed.

\section{VARIABIES}

b

$\mathrm{c}_{12}$

$\ddot{\mathbf{c}}$

c

F

$\underline{\mathbf{F}}$

$\underline{\mathbf{f}}$

G

$\underline{G}$

$\mathbf{g}$

$\mathbf{g}$

H

$\mathrm{H}_{1}^{2}$

$\underline{\mathbf{b}}$

\section{DESCRIPTION}

Wing span

Gain scheduling coefficient

Partial derivative of the nondimensional coefficient of force or moment 1 with respect to the nondimensional variable 2. (scalar)

Mean aerodynamic chord Gain scheduling coefficient System matrix. Aerodynamic contact force vector

Vector-valued nollinear function

Control input matrix

Thrust moment vector

Magnitude of $g_{L}(g=|g|)$

Gravitational acceleration vector

Altitude (sialar)

Transfer function matrix

Euler angle transformation from Frame 1 axes to Frame 2 axes

Angular nomentum vector 


\section{LIST OF SYMBOLS (Continued)}

VARIABLES

I

I

J

$J$

K

$\mathbf{k}$

L

M

$\underline{M}$

m

$N$

$\mathrm{n}_{\mathrm{z}}$

P

p

?

q

\section{DESCRIPTION}

Qotational inertia matrix

Identity matrix

Product or moment of inertia

(with appropriate subscripts)

Cost functional

State derivative premultiplying matrix

Gain (matrix)

Axis transformation matrix

for complete state vector

Gain (scalar)

Aerodynamic moment about the $x$-axis (scalar)

Angular rate transformation matrix

Aerodynamic moment about the $y$-axis (scalar)

Modal matrix composed of eigenvectors

Aerodynamic contact moment vector

Mass of the vehicle

Gain scheduling independent variable

Aerodynamic moment about the z-axis (scalar)

Load factor

Riccati matrix

Pole of a system

Rotational rate about the body $\mathrm{x}-\mathrm{ax}$ is

Melghting matrix

Rotational rate about the body $y$-axis 


\section{LIST OF SYMBOLS (Continued)}

\section{VARIABLES}

$q_{\infty}$

R

$r$

S

$\mathbf{s}$

$\mathrm{T}$

$\underline{T}$

$t$

$\underline{\mathrm{u}}$

u

V

$\mathbf{v}$

$\mathbf{v}$

w

$\mathrm{X}$

$\underline{\mathbf{x}}$

$\Delta \bar{x}$

X.I

$\mathrm{x}$

\section{DESCRIPTION}

Free stream dynamic pressure $\left(\frac{1}{2} \rho V^{2}\right)$

Weighiing matrix

Rotational rate about the body z-axis

Reference area (usually wing area)

Laplace transform variable

Thrust force magnitude ( $T=|\underline{T}|)$

Thrust force vector

Time

Control vector

Body $x$-axis velocity component

Element of control vector

Inertial velocity magnitude $(\mathrm{V}=|\underline{V}|)$

Velocity vector of body observed from inertial axes

Body $y$-axis velocity component

Body z-axis velocity component

Aerodynamic force along the $x$-axis (scalar)

State vector

Distance between actual c.g. location and point used for aerodynamic moment measurements

Inertial position vector

Position along the $x$-axis

Element of state vector 


\section{LIST OF SYMBCLS (Continued)}

\section{VARI ABLES}

Y

$y$

y

$\mathrm{Z}$

$\underline{z}$

$z$

VARIABLES (Greek)

$\alpha$

$\beta$

$\Gamma$

$\gamma$

sa

$\delta \mathrm{f}$

f

$\delta$

dSB

$\delta_{\mathrm{T}}$

$\zeta$

$\theta$

$\lambda$

$\Delta \mu$
DESCRIPTION

Aerodynamic force along the $y$-axis

Norm' l mode state vector

output vector

Position along the $y$-axis

Aerodynamic force along the $z$-ax is

Eigenvector

Position along the $z$-axis

Zero of a transfer function

\section{DESCRIPTION}

Wind-body pitch Fuler angle (Angle of Attack)

Negative of wind-body yaw Euler angle (Sideslip angle)

Controllability test matrix Inertial-velocity axis pitch Euler angle (Flight path angle)

Aileron deflection

Flap/slat deflection

Horizontal tail deflection

Rudder deflection

Speed brake deflection

Tr.rust command

Damping ratio

Inertial-body pitch Euler angle

Eigenvalue

Euler angle perturbation vector expressed in an orthogonal frame 


\section{LIST OF SYMBOLS (Continued)}

VARIABLES (Greek)

$\mu$

$\underline{v}$

$\xi$

$p$

$c$

$\phi$

$\psi$

$\underline{\omega}_{1}^{2}$

$\omega$

$w_{n}$
DESCRIPTION

Relative density

orientation or velocity magnitude and orientation vector

Inertial-velocity axis yaw Euler angle (Heading angle)

Air ; isity

Correlation coefficient

Real part of eigenvalue

Inertial-body roll Euler angle

Inertial-body yaw Euler angle

Fotational rate vector of Reference Frame 2 vith respect to

Reference Frame 1 and expressed

in Frame 1 coordir. es. ( $\underline{\omega}_{1}^{2}=\mathrm{H}_{2}^{1} \underline{\omega}_{2}^{1}$ so $\underline{\omega}_{2}^{1}$ is left-handed.

Thus, Frame 1 and Frame 2 are not interchangeable.)

Frequency (Imaginary part of eigenvalue)

Natural frejuency

VARIABLES (Subscripts or Superscripts)

DESCRIPTION

B

DR

dyn

F

I

1

m
Body axes

Pertaining to Dutch rull mode Stability axis derivative

Final value

Inertial axes

Initial value

Aerodynamic moment about the $x$-axis

Aerodynamic moment about the $y$-axis 


\section{LIST OF SYMBOLS (Continucd)}

VARIABLES (Subscripts or Superscripts)

n

roll

W

$\mathrm{X}$

$\mathrm{x}$

$\mathrm{Y}$

y

$\mathrm{Z}$

$Z$

\section{PUNCTUATION}

( )

$(\sim)$

()$^{\mathrm{T}}$

\section{DESCRIPTION}

Aerndynamic moment about the $z$-axis

pertaining to roll mode

Wind axes

Aerodynamic force atong the $\mathrm{x}$-axis

Component along the $x$-axis

Aerodynamic force along the $\mathrm{y}$-axis

Component along the $y$-axis

Aerodynamic force along the z-axis

Component along the $z$-axis

Time derivative - occurs after any transformation unless explicitly indicated otherwise

Matrix equivalent to vector cross product. Specifically, if $x$ is the three-dimensional vestor

$$
\underline{x}=\left[\begin{array}{l}
x \\
y \\
z
\end{array}\right]
$$

then

$$
\tilde{x}=\left[\begin{array}{rrr}
0 & -z & y \\
z & 0 & -x \\
-y & x & 0
\end{array}\right]
$$

the crosis product of $x$ and another vector ( $F$, for example) is equal to the product of the matrix $\tilde{x}$ and the vector $\hat{F}$.

$$
\underline{x} \times \underline{F}=\tilde{x} F
$$

Transpose of a vector or matrix 


\section{LIST OF SYMBOLS (Continued)}

\section{PUNCTUATION (Continued)}

(^)

( )

( ) $)^{-1}$

( ) 0

$\Delta(\quad)$

ACRONYM

ARI

ARDP

C.E.

DPSAS

IAS

LCDP

\section{DESCRIPTION}

Scheduled or estimated value

Mean value

Inverse of a matrix

Reference or nominal value of a variable

Perturbation about the nominal value of a variable

\section{DEFINITION}

Aileron-rudder-interconnect

Acceleration response departure parameter

Center of gravity

Departure-prevention stability augmentation system

Indicated air speed

Lateral control departure parameter 
STABILITY AND CONTROL

OF MANEUVERING HIGH-PERFORMANCE AIRCRAFT

Robert F. Stengel and Paul W. Berry

The Analytic Sciences Corporation

1.

INTRODUCTION

\subsection{BACKGROUND}

As aircraft become capable of flying higher, faster, and with more maneuverability, prevention of inadvertent departure from controlled flight takes on added significance. To some extent, the airframe can be designed to provide inherent protection against loss of control, as in the addition of nose strakes to regulate high angle-of-attack $(\alpha)$ vortices; however, performance objectives are likely to dominate the choice of such features as wing planform and chord section, nose shape, aircraft density ratic, and tail area. It is likely, therefore, that the freedom to configure the aircraft for intrinsic departure prevention will be restricted and that the flight control system will be called upol to provide additional protection.

Flight at high $\alpha$ invariably complicates the control problem. Dynamic coupling between longitudinal and lateraldirectional motions becomes apparent, aerodynamic trends vary considerably, and control surface effects diminish or become adverse. Coupling and nonlinearities can cause a selfsustained oscillation ("wing rock") at high $\alpha$, degrading precision tracking tasks without necessarily causing loss of control. Abrupt maneuvering, external disturbances, control system failure, or pilot error can produce a "departure" (pitch, yaw, or roll divergence), possibly leading to high acceleration and to a fully developed spin. The recovery from spin or gyration is, at best, an emergency procedure which is not always successful. Clearly, it is preferable to 
prevent the departure before it occurs rather than to be forced to take emergency measures.

Although departure and spin are related topics, there are at least as many dissimilarities between the phenomena as there are similarities. Departure is a transient event, while spin is a quasi-steady condition. Departure connotes instability with respect to the initial flight condition, while spin can be thought of as a bounded, periodic (and therefore stable) motion about a nearequilibrium flight path. Departure can occur in level flight, but spinning equilibrium ultimately results in vertical motion of the aircraft's center of gravity.

Departure and spin both are beset by the difficulties inherent in describing fully coupled dynamic systems of bigh order and in describing static and rotary aerodynamics at complex flight conditions. However, as suggested by the preceding comparison, the approximations and assumptions which hold for one are not necessarily appropriate for the other. In particular, it appears that linearized dynamic models may have a practical utility in preventing departure which does not readily carry over to spin recovery. The reason is that a closed-loop control law which continuously acts to prevent departure restricts ..ugular excursions to small values; hence, their dynamic effects can be described by linear models. A spin recovery strategy necessarily must operate with large angular changes which result in significant nonlinear effects.

The key to developing a linear model which is satisfactory for the study and control of departure is it the recognition that the nominal fight path, used as a reference for the variational (linearized) motions, need not 
represent a steady, unaccelerated flight condition. A flight path in dynamic equilibrium is equally satisfactory as long $a s i t$ is understood that variational motions are referenced to the continuously changing nominal flight,path. The interlediate step of linearizing the aircraft model with non-zero uut constant sideslip angle $\left(\beta_{0}\right)$, angular rate $\left(p_{0}, q_{0}, r_{0}\right)$, and load factor $\left(n_{z_{0}}\right)$ provides the coupling between longitudinal and lateral-directional motions which is so importint in the study of departure.

It is essential to recognize that the combined effects of non-zero mean motions lead to significant coupling which otherwise might be missed in a linear dynamic model. It has been demonstrated that large mean values of roll rate and sideslip angle separately produce significant coupling of the short period and Dutch roll modes. It is less well known that the combined effects of these two variables produce coupling which is qualitatively different from that induced by a single variable. This variability in stability effect is similar to the seeming unpredictability us the departure modes of some aircraft, in which the aircraft is known to have more than one departure mode for supposedly similar flight conditions. This also suggests that defarture modes are more predictable than might have been assu'ned.

The complexity of the coupled dynamics and the possivility for misinterpreted control cues at high $\alpha$ indicete a need for departure preventing control systems in isighly maneuverable aircraft. New developments are required in characterjiing the evolution of motions during extreme maneuvering and in the computation of contiol solutions. 
1.2 PURPOSE

The purpose of this investigation is to identify general rules for the design of departure-preventing control systems. It achieving this objective, the analytic foundations for linear-time-invariant modeling of aircraft dynamics are extended to include extreme maneuvering conditions. Using tools of linear systems analysis, the stability and control characteristics of a high-performance aircraft are examined over a wide range of flight conditions, and specific effects of configurational modification are developed. The study culminates in the development and evaluation of control laws for a Departure-Prevention Stability Augmentation System (DPSAS) using linear-optimal control theory.

\subsection{SUMMARY OF RESULTS}

The major tasks of this project were defined at the outset as:

- Dynamic Model Development

- Characterization of Departure Modes

- Controllability Effects on Aircraft Departure

- Control Laws for Departure Prevention

These tasks can be summarized briefly as follows: Dynamic Model Development provided a range of nonlinear and linear dynamic models for use in the analysis of departure and the design of DPSAS control laws. Characterization of Departure Modes addressed the unaugmented stability of high-performance aircraft. Controllability Effects on Aircraft Departure considered the direct (open-1ocp) effects of control forces on aircraft departure. Control Laws for Departure Prevention resulted in the design and simulation of linear-optimal 
regulator control laws which stabilize the reference aircraft during extreme maneuvering and which adapt to changing flight coidition.

Although aircraft equations of motion are developed in several texts, no derivatio which retains all coupling terms in the linearized equations of motion was found. Therefore, the present investigation began with the development of ronlinear equations in four axis systems (inertial or earth-relative, velocity, wind, and body;. This was followed by derivation of the associated linear equations of motion, as well as equilibrium equations which define a generalized trim condition. The validity of these equations was established by direct comparisons of the time responses of the linear and nonlinear equations.

A small, supersonic fighter aircraft was chosen as a baseline for study. A comprehensive model of subsonic nonlinear aerodynamic coefficients was available for this aircraft and was used to generate aircraft local stability derivatives as flight condition was varied. These stability derivatives formed a large part of the linear-timeinvariant dynamic model, which was analyzed by eigenvalue/ eigenvector, transfer function, and time response methods. Thus, the significant aspects of aerodynamic nonlinearity and inertial coupling, i.e., the local sensitivities to initial condition, disturbance, and control perturbations, were retained in the aircraft model.

Using the reference aircraft as a starting point, the analysis proceeded along two separate lines. The first approach was to assess the effects of maneuvers and flight condition on the reference aircraft, recomputing the linear model for each variation in nominal angle, angular rate, 
altitude, and velocity. In order to distinguish between aerodynamic and purely inertial effects, a limited number of cases were evaliated with varying flight condition and fixed aerodynamic derivatives. The second approach was to vary individual coefficients of the linear model so that specific configurational effects could be analyzed. The quantitative results presented here strictly apply only to the specific configurations studied; hence, care should be exercised in generalizing these results to other configurations.

The Iinear-optimal regulator was applied to the DPSAS design problem, and the present results demonstrate the substantial benefits offered by the linear-optimal controllers. A design procedure which also identifies gaiıscheduling relationships is presented; it has the following features :

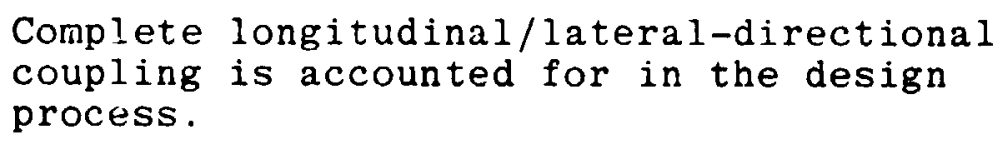

- All significant feedback gains, crossfeeds, and con \%rol interconnects are identified.

- The control structure is guaranteed to stabilize the aircraft, assuming that aircraft parameters are known and motions are measured precisely.

- Tradeoffs between control authority, control power, and aircraft notions are incorporated in the design piocess.

- The DPSAS adapts to varying flight condition.

The extension of this design procedure to a, full command augmentation system is direct, as the control design algorithms are easily restructured to consider handing quali ties requirements, control-actuator rate limits, noisy feedback measurements, and digital impiementation. 


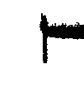
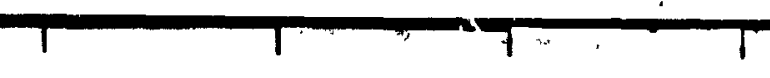

In the process of conducting this investigation, a flexible computer program (AIPHA) for the analysis of high angle-of-attack stability and control was developed. Program ALPHA generates linear dynamic modeis and trim conditions from nonlinear aerodynamic and inertial data; presents results for body-axis, stability-axis, and reduced-order models; computes eigenvalues, eigenvectors, transfer functions, departure parameters, and linear-optimal control gains; calculates time histories for various initial condi.. tions, control inputs, and disturbances; and incorporates a logical (executive) structure which facilitates parameter sweeps, initial condition variations, and model modifications during a single computer run.

\subsection{ORGANIZATION OF THE REPORT}

This report presents dynamic equations, stability and control charactelistics of high-performance aircraft, and control laws for departure prevention. Prior results related to extreme maneuvering of aircraft are reviewed in Chapter 2, which then presents a validation of the linear model and describes the effects of extreme maneuvering on the dynamics of the reference aircraft. Configurationai effects on maneuvering rynamics are discussed in Chapter 3. Control laws for a Depirture-Prevention Stability Augmentation System (DPSAS) are derived in Chapter 4, and the report is concluded by Chapter 5. Appendix A is directed to the development of nonlinear equations of motion, linear equations, generalized trim conditions, and tools for linear systems anaiysis. The model for the reference aircraft is summarized in Appendix B. 


\section{DYNAMIC CHARACTEF.ISTICS OF HIGH-PERFORMANCE AIRCRAFT}

\subsection{OVERVIEW}

The problems associated with extreme maneuvering have two common characteristics: loss of control and large angles and/or angular rates, i.e., angles and rates generally beyond the range of normal, " $1-\mathrm{g}$ " flight operations. Extreme maneuvering difficulties fall in the following categories, which contain some overlap:

- Decreased inherent stability

- Degraded handling qualities

- Longitudinal/lateral-directional coupling

- Stall

- Wing rock

- Departure

- Post-stall gyrations

- Incipient spin

- Fully evolved spin

The ordering of this list suggests that the severity of these phenomena increases with angle of attack and is aggravated by angular rates and sideslip ante.

Appendix A presents a formal development of fully coupled Iinear-time-invariant models for aircraft motion. These models are suitable for investigatins perturbation motions which are referenced to large angles and large angular rates. After reviewing prior investigations of aircraft dynamics in Section 2.2, the remainder of the chapter concerns the application of linear systems analysis to the stability and control of a high-performance aircraft 
(which is described in Appendix B). Section 2.3 compares the time responses of linear and nonlinear dynamic models. Section 2.4 is directed at aircraft stability, and Section 2.5 treats aircraft control. Variations in the aircraft's dynamic characteristics during extreme maneuvering are addressed in Section 2.6, which also introduces rudimentary effects of the pilot's control actions while executing a tracking task. The chapter is summarized in Section 2.7.

2.2 PRIOR STUDIES OF AIRCRAFT AT EXTREME FLIGHT CONDITIONS

Although published studies of aircraft dynamics shortly followed the Wright irothers' flight (Ref. 1), ind the concept of stability derivatives was published in 1913 (Ref. 2), the dynamics of aircraft which are executing extreme maneuvers received littie attention until the late 1940's. (Investigation of the related problem of aircraft spinning had begun a decade earlier.) There are several reasons for this, but the most significant reason is that extreme maneuvers had not presented sufficient problems to merit detalled engineering study. The advent of fighter aircraft with higher speeds, higher roll rates, higher density, lower inherent damping, and higher cost accentuated the importance of understianding extreme maneuvering dynamics. Furthermore, the improved analytical tools and techniques spawned by World War II became available for application to flight dynamic problems.

In addition to the extensive flight testing which high-performance aircraft received, three fundamentally different avenues have been followed in the investigation of maneuvering flight. The first approach is the study of rigid-body dynamics of the aircraft, the second is the study of aerodynamics, and the third is the study of control. The 
P1rst two areas have a cause-and-effect relationship - aerodynamic forces modify the momentum and energy of the airplane -- and there is "feedback," in the sense that the changing velocity and attitude of the vehicle contribute to changes in the aerodynamic forces. Although dynamic problems result from the interaction of dynamics and aerodynamics, one can distinguish between these two areas in reviewing past work. The third area considers methods of augmenting the natural aircraft stability, of limiting excursions from the normal flight regime, of providing adequate response, and of recovering from fully evolved spins.

\subsubsection{Dynamics of the Aircraft}

The objective of study is the solution of nonlinear and linear equaticns of motion, e.g., those derived in Appendix A. Options for analysis can be classified as explicit, in which a direct solution of motion equations is sought, or implicit, in which the evolution of motions is inferred from characteristics of the system. The solution of these equations describes the aircraft's response to initial conditions and disturbances, and it provides a basis for identifying control policies. The stability of the solution describes its tendency to return to a nominal value. Given an initial disturbance, the stable aircraft's solution returns to the nominal solution (or its error is, at least, bounded); the unstable aircraft's solution diverges. These analytical methods can be summarized as follows:

Explicit Analysis - Stability, Response, and Control

- Analog integration of differential equations

- Numerical integration of differential equations

- Closed-form solution of differential equations

- Equilibrium solution of algebraic equations 
Implicit Analysis - Stability

- Generalized energy balance (Liapunov method)

- Absolute stability bounds (Popov criterion, circle criterion, etc.)

- Stability bounds of "classical" differential equations (Mathieu's equation, etc.)

- Eigenvalue analysis (Routh-Hurwitz criterion, root locus, etc.)

- Quasi-linear Eigenvalue analysis

Implicit Analysis - Response and Control

- Eigenvector analysis

- Time-domain methods (Impulse or indicial response, auto- and cross-correlation functions, etc.)

- Transform methods (Frequency response, transfer functions, spectral density, etc.)

Applications of some of these techniques to the maneuvering flight problem are documented in the literature. Much of the work related to handling qualities, stability, coupling, and departurc is based upon linear-timeinvariant models and uses eigenvalue and transfer function analysis. Work of this type is reported in Refs. 3 to 10. In addition, parameters of linear-time-invariant models

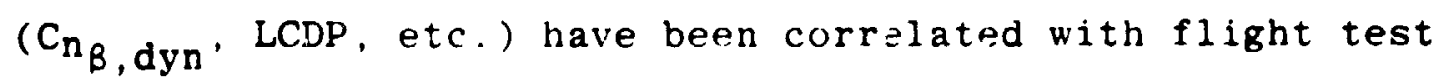
or piloted simulation data using little or no direct analysis of the equations of motion (Refs. 11 to 14). Quasilinearization of a significant sicieslip nonlinearity is applied to the wing rock problem in Ref. 15, and closed-form solutions for a class of large maneuvers are presented in Ref. 16. Since the early 1960's, a large number of investigaticns harr usea analng and numerical integration in the 
study of departure, stall, post-stall gyrations and spin (Refs. 17 to 27). Equilibrium solutions of nonlinear equations of motion have been used to determine spin conditions and are discussed in Refs. 24 and 28 to 30 .

A number of linear-timo-invariant departure parameters have been identified, as reported in Refs. 5, 13, and 14. These parameters relate to transfer function numerators and $d$ rominators and are expressed in terms of stability and control derivatives $\left(c_{n_{\beta}}, c_{1_{\beta}}, c_{n_{\delta_{a}}}, c_{1_{\delta_{a}}}, c_{n_{\delta_{r}}}, c_{1_{\delta_{r}}}\right)$, angle of attack $\left(\alpha_{0}\right)$, moments of inertia $\left(I_{x}, I_{z}\right)$, directional stability augmencation gain $\left(k_{1}\right)$, and ailer a.rudderinterconnect (ARI) gain $\left(k_{2}\right)$ :

Directional Staility Parameter

$$
C_{n_{B, d y n}}=C_{n_{\beta}} \cos \alpha_{0}-\frac{I_{z}}{I_{x}} C_{1_{B}} \sin \alpha_{0}
$$

\section{Lateral Control Departure Parameter}

$$
\operatorname{LCDP}=C_{n_{B}}-C_{1_{B}} \frac{c_{n_{\delta_{a}}}}{C_{1_{\delta_{a}}}}
$$

Augmented Lateral Control Neparture Parameter

$$
\operatorname{LCDP}_{A}=c_{n_{B}}-c_{1_{B}} \frac{c_{n_{\delta_{a}}}}{C_{1_{\delta_{a}}}}+k_{1}\left(\frac{c_{n_{\delta_{a}}}}{C_{l_{\delta_{a}}}} c_{1_{\delta_{r}}}-c_{n_{\delta_{r}}}\right)
$$

ARI Lateral Contrul Departure Parameter

$$
\operatorname{LCDP}{ }_{A R I}=c_{n_{B}}-c_{1_{B}}\left(\frac{c_{n_{\delta_{a}}+k_{2}} c_{n_{\delta_{r}}}}{\left.c_{1_{\delta_{a}}+k_{2} c_{1_{\delta_{r}}}}\right)}\right.
$$




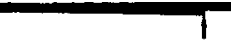

Acceleration Response Departure Parameter ("B plus $\delta$ Stability Indicator")

$$
\begin{aligned}
& \operatorname{ARDP}_{B}=a_{O^{-}} \tan ^{-1}\left(\frac{\mathrm{C}_{n_{B} I}}{\mathrm{C}_{1_{B} I_{z}}}\right) \\
& \operatorname{ARDP}_{\delta}=\alpha_{0^{-}} \tan ^{-1}\left(\frac{\mathrm{C}_{\mathrm{n}_{\delta}}}{\mathrm{C}_{1_{\delta}}} \frac{\mathrm{I}_{\mathrm{x}}}{\mathrm{I}_{\mathrm{z}}}\right)
\end{aligned}
$$

The first four criteria indicate resistance to departure when their magnitudes are greater than zero, while the last requires $\mathrm{ARDP}_{B}$ to be greater than zero and greaier than $\mathrm{ARDP}_{\delta}$. The first criterion relates to the open-loop static stability of the Dutch roll mode; $C_{n_{B} \text {, dyn }}>0$ is an approximate requirement for stability. The LCDP's are approximations to the closed-loop static stability of the Dut.ch roll mode when lateral control is used to maintain constant roll rate; when they al a greater than zero, the Dutch roll mode is iaticaliy stable, but when they are less than zero, the Dutch roll mode is statically unstable. The " $\beta$ plus $\delta " c r i-$ terion is an attempt to combine stability and control considerations in a single departure indicator.

There are a number of inadequacies in the above parameters, although they provide insight for future developments. They are approximations to the exact transfer function coefficients and do not indicate actual pole-zero locations; they neglect dampir: terms entirely; and they do not accrunt for longitudinal/lateral-directional coupling induced by large, sideslip angle $(\beta)$ and angular rates $(p, q, r)$-in fact, the longitudinal dynamics are ignored completely. References $8,9,31$, and 32 introduce coupling effects due to $\beta$, illustrating the importance of some of the neglected terms, and Ref. 33 treats the dynamics of steady turning flight. 

extreme maneuvers at subsonic velocity, in which case Mach number effects may not be significant; however, scaling of the flow to provide representative Reynolds number is required, if model test data is to be applied to the rull-scale aircraft.

Stability problems associated with large aerodynamic angles may arise fyom either the nose, wing, or tail, depending on aircraft configuration. Consequently, it is impossible to identify a single acrodynamic solution to problems of departure (other than to make all aircraft use the same configuration). Aerodynamic solutions include wing-root leadingedge extensions, nose strakes, redesign of the nose crosssection and profile, maneuvering (leading-edge) flaps, and adjustment of horizontal tail anhedral.

The aerodynamic forces and moments discussed above are static, in that they arise from fixed values of $\alpha$ and $B$. These terms establish the static stability and trim points of the aircraft. Forces and moments which result from angular rates $(p, q, r)$ and accelerations $(\dot{u}, \dot{v}, \dot{w}, \dot{p}, \dot{q}, \dot{r})$ are dynamic and thus contribute to damping and transient response. There is indication that assumptions which corventionally are made for low-angle flight conditions, e.g., that the $\dot{\beta}$ and yaw-rate effects are simply additive, break down at high angles. Unfortunately, dynamic forces and moments are difficult to measure in practice, and relatively few facilities are equipped to maasure dynamic forces, much less to separate $\dot{\varepsilon}$ and $r$ effects.

\subsubsection{Control}

The third subject for study is control of flight motions during rapid maneuvering, and it is clear that the emphasis of recent studies has shifted away from spin 
recovery to departure and spin prevention. At best, spin recovery is ar emergency procedure, and it is not always successful. Safety is important, but it is not the only issue: an aircraft which is prone to spin is less likely to complete its mission successfully. It is preferable, therefore, to prevent the spin before it occurs.

Nevertheless, if a spin occurs, it is important to understand what control actions can be used to recover. The most favored technique for recovery is to command constant, anti-spin control settings (Ref. 47). The proper control settings depend on the type of spin (flat, steep, oscillatory, or erratic) and on the aircraft configuration--particularly the tail damping, aircraft density, and mass distribution. In many cases. the available anti-spin control moment is less than the restoring moments which maintain spin equilibrium, i.e., the spin cannot be broken with constant zontrol settings. The idea of "resonating" the aircraft out of the spin by applying oscillatory controls was proposed as early as 1931 (Ref. 48) and as recently as 1974 (Ref. 49). While this task may be difficult for the pilot to execute, simple logic for pulsing the controls automatically can be designed.

The concept of automatic control systems which prevent stall, departure, and spin has gained momentum, and it is now recognized that departure prevention can be built into the stability augmentation system (SAS), which virtually all modern high-performance aircraft contain. The basic approaches to departure prevention taken to date can be classified as limiters (or inhibitors), staility aug= menters, control incerconnects, or some combination of these three. A dual-mode spin-prevention system is developed in Ref. 50. This system applies constant antispin controls 
when $\alpha$ and $r$ exceed separate threshold values, then switches to a rate-damping mode once the spin is neutralized. Reference 51 presents a departure-prevention system which inhibits $\alpha$, increases directional stiffness (by stability augmentation), and restricts the aircraft to roll about its flight path. A stall-inhibitor system for a variable-sweep aircraft is described in Ref. 52. This system incorporates an a limiter, $x$-dependen i command-and stability-augmentation gains, increased directional stiffness and danping, and ailaron-rudder interconnect. A similar philosophy is adopted in Ref. 53, where speed stability also is augmented to account for $a-1$ imiting effects in the landing approach. Departure prevention considerations are evident in the designs for two additional high-performance aircraft (Refs. 54 and 55), and the effects of stability augmentation and roll/yaw interconnect are demonstrated in Ref. 56 .

While a common thread runs through the designs $1 \geq-$ ported in Refs. 50 to 56 , these reports suggest the need for a unifying control theory to aid the design of future departure prevention systems. These studies have made extensive use of experience, nonlinear simulation, and flight testing to arrive at successful designs, but the underlying concepts of stability, response, and control remain to be identified.

Summary - This section has presented a brief survey of prior developments related to maneuvering flight, distinguishing between investigations of dynamics, aerodynamics, and control of the aircraft. It is shown that the range of problems, from degraded handling qualities to fully evolved spin, can not be completely solved by focusing on only one area. New developments are required in characterizing the evolution of motions; in the measurement and understanding of forces and moments at extreme flight conditions; and in 
the computation of control solutions. In following sections of this report, the problems of dynamics and control are addressed in detail.

\subsection{COMPARISON OF RESULTS FROM LINEAR AND NONLINEAR SIMULATIONS}

The use of linear models in highly dynamic situations has been restricted, in the past, by a lack of linear models which include complete dynamic effects and by the lack of a general method of finding the proper nominal flight condition. The linear models developed in Appendix A include all the effects of a dynamic nominal flight condition. To verify these models and to develop methods of using them, this section presents a comparison of linear and nonlinear results. The nonlinear results are in he form of test trajectories generated by a nonlinear aircraft simulation using aerodynamic and mass data for the reference aircraft.

During the early part of this investigation, large differences between the linear and noulinear results appeared along highly dynamic flight trajectories. These were traced to the use of an incorrect nominal state vector. From these observations, the concept of generalized trim (Section A.3.2) was developed, and a method of finding generalized trim points was derived. (Section A.3.2 describes the generalized trim calculation computer program.)

The generalized trim condition is one in which the derivatives of the velocity and angular rate states are as close to zero as possible. Dimensionality considerations, as discussed in Section A.3.2, lead to the conclusion that the generalized trim problem involves six of the aircraft 
state equations, the correspoiding six states, and six control parameters (in this case, four control settings and two Euler angles). The problem becomes a search for those values of nominal body-axis velocities and angular rates that null tho selected nominal state rates.

The following subsections examine specific results of the comparison of linear and nonlinear trajectories to support these points.

\subsubsection{Elevator Contro1 Input}

Elevator deflection produces a change in pitch moment, causing an immediate change in the aircraft angle of attack. This causes the aircraft to climb or dive. In combination with the throttle, elevator position establishes the aircraft flight speed, angle of attack, and flight path angle. The tests presented here involve small-amplitude elevator inputs when the aircraft is in straight-and-level flight at slow speed and high angle of attack. Figure 2.3-1 illustrates the time history of the most important longitudinal motion variables for eight seconds following the control application. All lateral variables are approximately zero for the nonlinear model and exactly zero for the linear model.

Comparison of the linear and nonlinear curves indicates excellent agreement. It is important to note that the nonlinear aircraft response verifies that the lateral and iongitudinal modes are truly uncoupled in this flight condition. The nominal flight condition is a steady-trim flight condition and satisfies the generalized trim condition. 

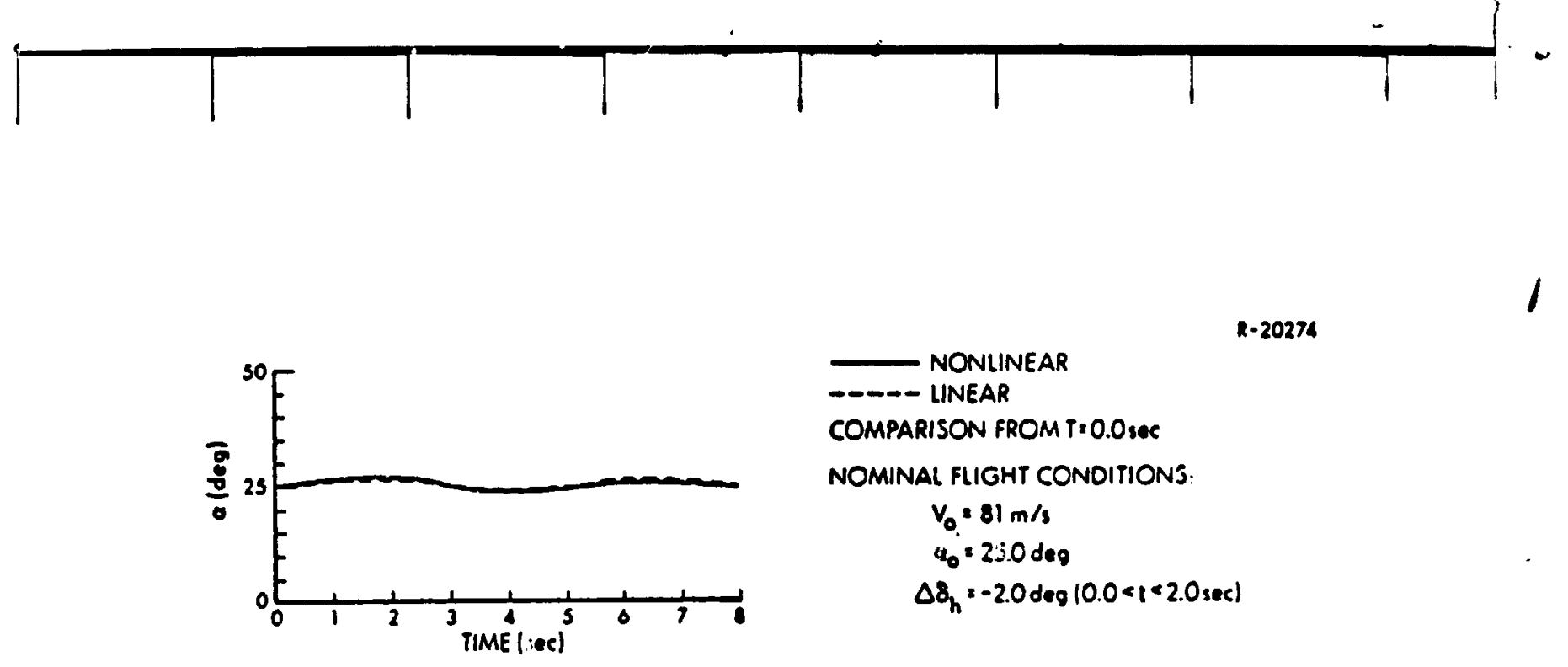

$R-20274$ NOMINAL FLIGHT CONOITIONS:

$$
\begin{aligned}
v_{0} & =81 \mathrm{~m} / \mathrm{s} \\
q_{0} & =25.0 \text { deg } \\
\Delta 8_{h} & =-2.0 \text { deg }(0.0<1<2.0 \mathrm{sec})
\end{aligned}
$$

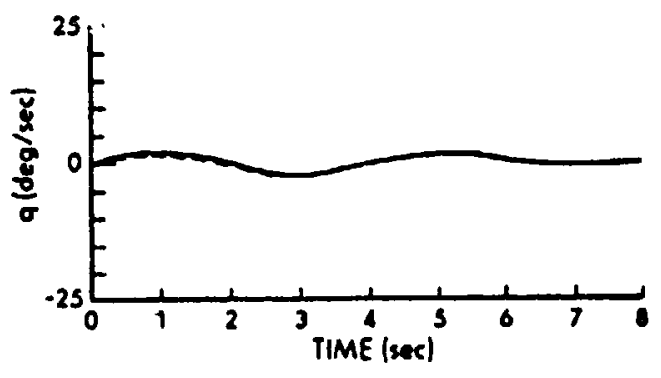

Figure 2.3-1 Small Anplitude Elevator Input

\subsubsection{Aileron Control Input}

The ailerons primarily provide roll moment, and the trajectories shown in Fig. 2.3-2 illustrate the aircraft response to a small amplitude alleron doublet. The linearized trajectory, whose nominal flight condition is again straigtt-and-level flight, differs only slightly from the true nonlinear response, and the linear and nonlinear trajectories exhibit lateral-longltudinal separation.

\subsubsection{Rudder Control Input}

Large-input, large-response traiectories resulting from rudder deflection are examined in this subsectiou, with the goal of testing the trajectory matching capabilities of a linear simulation for a highly dynamic flight condition. The noulinear test trajectory lasts eight seconds after the 

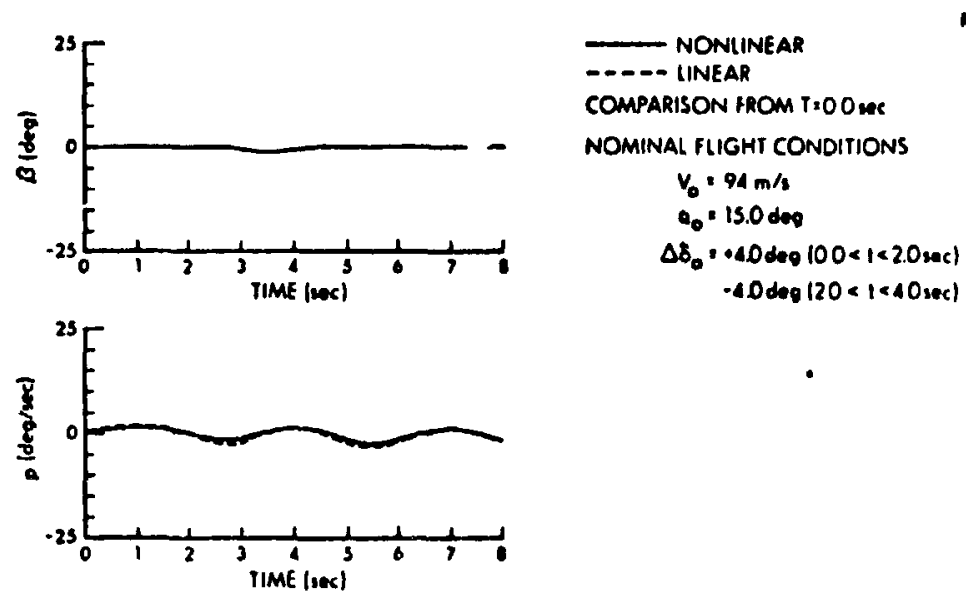

$-40 \operatorname{deg}(20<1<10$ iec $)$

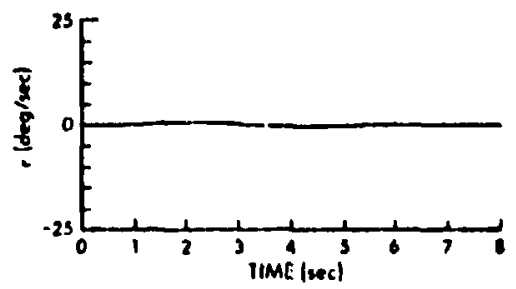

Figure 2.3-2 Small Amplitude Aileron Input

control is applied; linear trajectories starting at the initial time and at four seconds into the trajectory are tested.

Figure 2.3-3 compares the nonlinear trajectory to a linear trajectory starting at the time of control application. The nominal trajectory for linearization is the original static triui $f l i g h t$ condition of straight-and-level fight. The trajectory match is acceptable for aivut two seconds, and the angle-of-attack plot illustrates the cause of the deviation. Because it exhibits lateral-longitudinal separation, the linear trajectory does not capture the change in angle of attack that the nonlinear trajectory contains. This change in angle of attack has a large effect on the subsequent dynamics which the linear model fails to duplicate. 

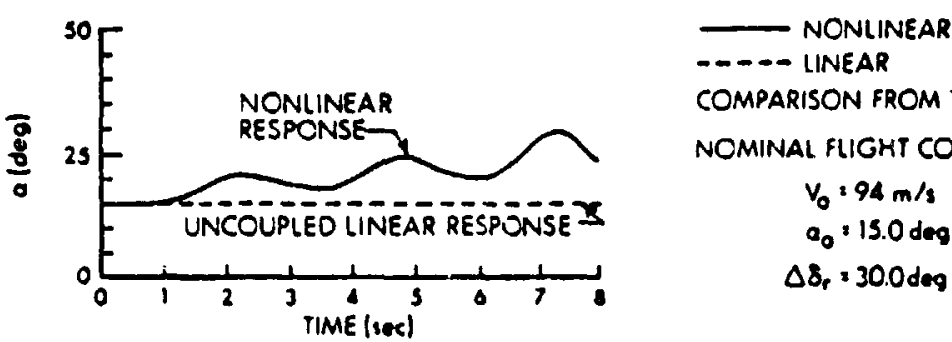

COMPARISON FROM $T=0.0$ see

NOMINAL FUIGHT CONDIIONS

$$
\begin{aligned}
& V_{0}=94 \mathrm{~m} / \mathrm{s} \\
& Q_{0}=15.0 \text { deg }
\end{aligned}
$$

$\Delta \delta_{r}=30.0 \mathrm{deg}(1>0.0 \mathrm{sec})$
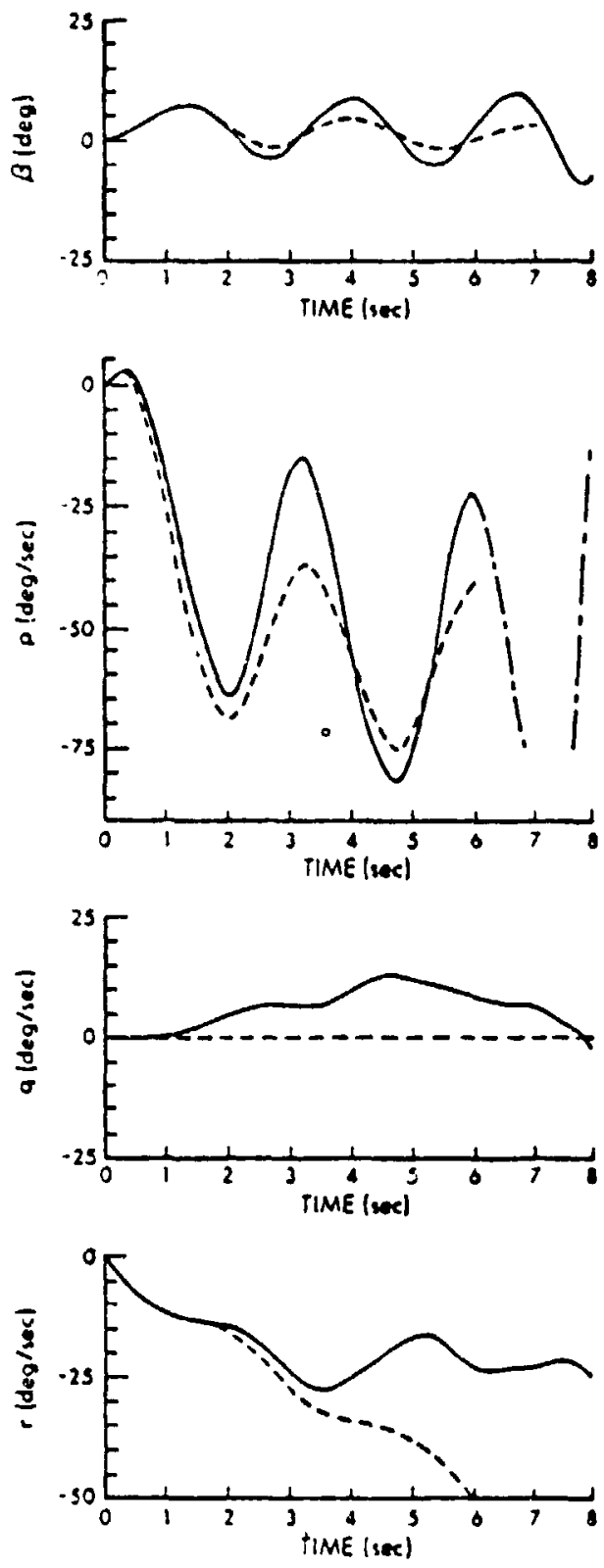

Figure 2.3-3 Large Amplitude Rudder Input -Comparison of Initial Response 
By examining the trajectory beginning four seconds after the control is applied, methods of linearization for highly dynamic trajectories can be derived. Figure 2.3-4 illustrates an early attempt. Here, the point of linearization is approximate, i.e., it does not satisfy the generalized trim condition discussed below and in Appendix A. The resulting linear trajectory diverges from the nonlinear trajectory fairly quickly, and the slopes do not match at the initial point for some states. Furthermore, the frequency of the resulting motion is considerably different from that of the nonlinear motion. Due to its dependence on ad hoc estimation of the nominal flight condition, the results of this approach are highly variable in quality.

One of the most striking errors in the linear trajectories shown in Fig. 2.3-4 is that the slopes of the states do not match at the beginning of the linear trajectory. This observation, which implies that the nominal state rates are not zero, led to the development of the generalized trim concept. In this context, this concept indicates that to provide an accurate representation of a nonlinear system by a linearized one, it is necessary to choose a point of linearization that exhibits zero nominal state rates.

Applying this Generalized trim procedure to the point four seconds after control application produces the results shown in Fig. 2.3-5. Compared to the previous figure, the generalized trim procedure produces clearly superior results. There are no initial slope errors evident, the match is excellent for two seconds, and it is reasonably close for much longer. Additionally, the frequency of the linearized motions is close to that of the nonlinear 

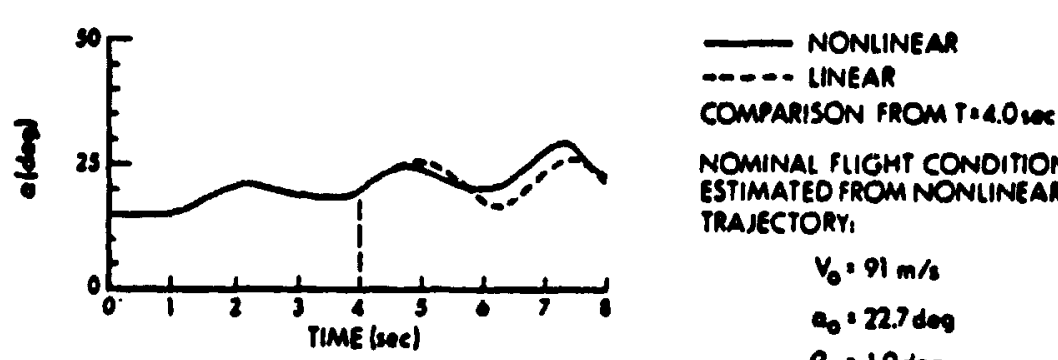

1.20078

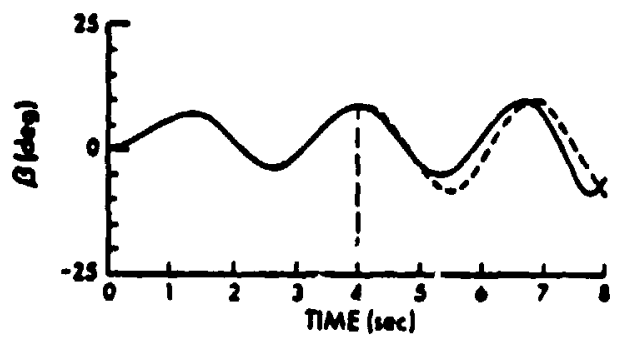

NOMINAL FLIGHT CONOIIION IS

ESTIMATEO FROM NONUINEAR

TRAJECTORY,

$$
\begin{aligned}
& V_{0}=91 \mathrm{~m} / \mathrm{s} \\
& \omega_{0} \cdot 22.7 \mathrm{deg}
\end{aligned}
$$

$B_{0}=1.0 \mathrm{deq}$

po" - $50.0 \mathrm{deg} / \mathrm{ser}$

$90:-10.0 \mathrm{deg} / \mathrm{wec}$

$r_{0}=20.0$ deg/me

$8_{\text {re }} \cdot 30.0$ des
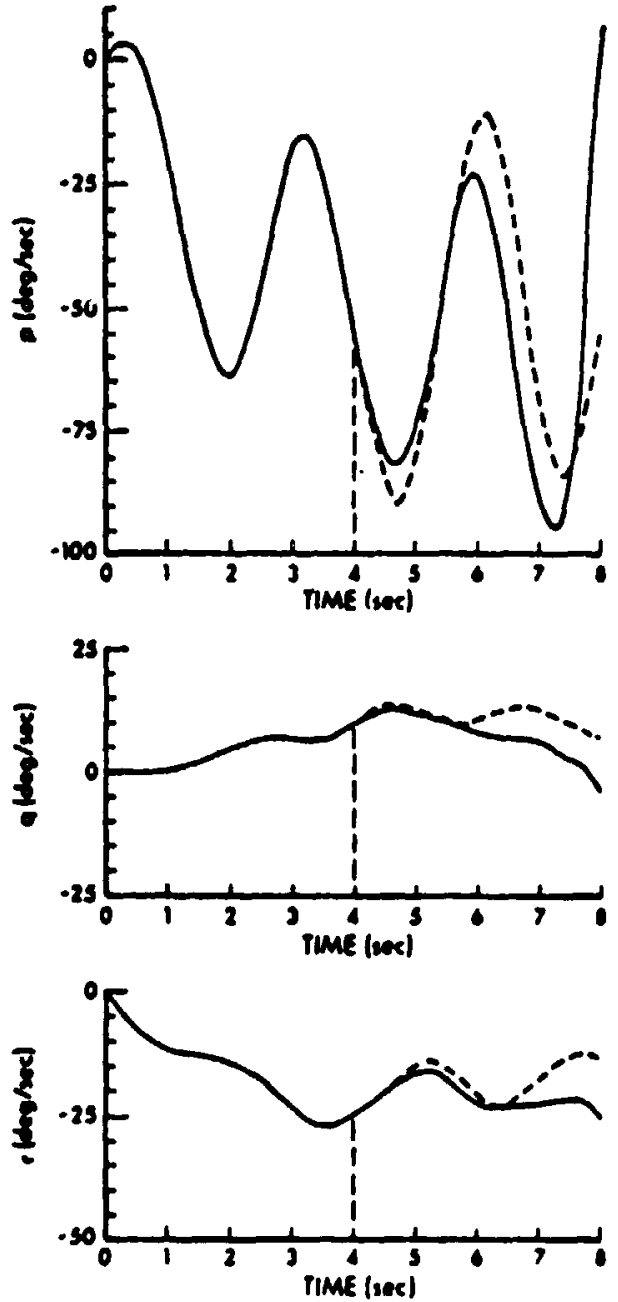

Figure 2.3-4 Large Amplitude Rudder Inpist -- Comparison of Evolved Response $\mathrm{Wlth}$ Ad Hoc Reference Point for Linearization 


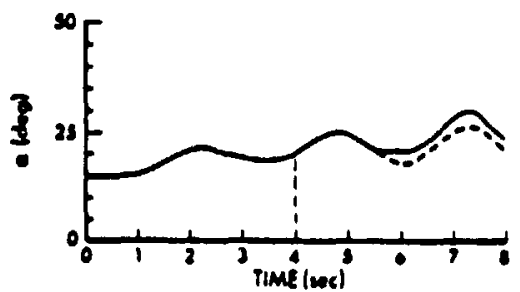

- NONINEAR

-.--- LINEAR

COMPARISON PROM Ti.1OME

NOMINAL FLIGHI CONDITION

is CALCULATED OT GENERALIZEO

ralm.

$\mathrm{V}_{0} \cdot 91 \mathrm{~m} / \mathrm{s}$

0.22 94o

$B_{0}+1.0$ deg

$D_{0}+-317$ argluer

$c_{0} \cdot 10 S \mathrm{deg} / \mathrm{wac}$

$10 \cdot-20.0 \mathrm{cog} / \mathrm{mec}$

$8,1300 \mathrm{dmp}$
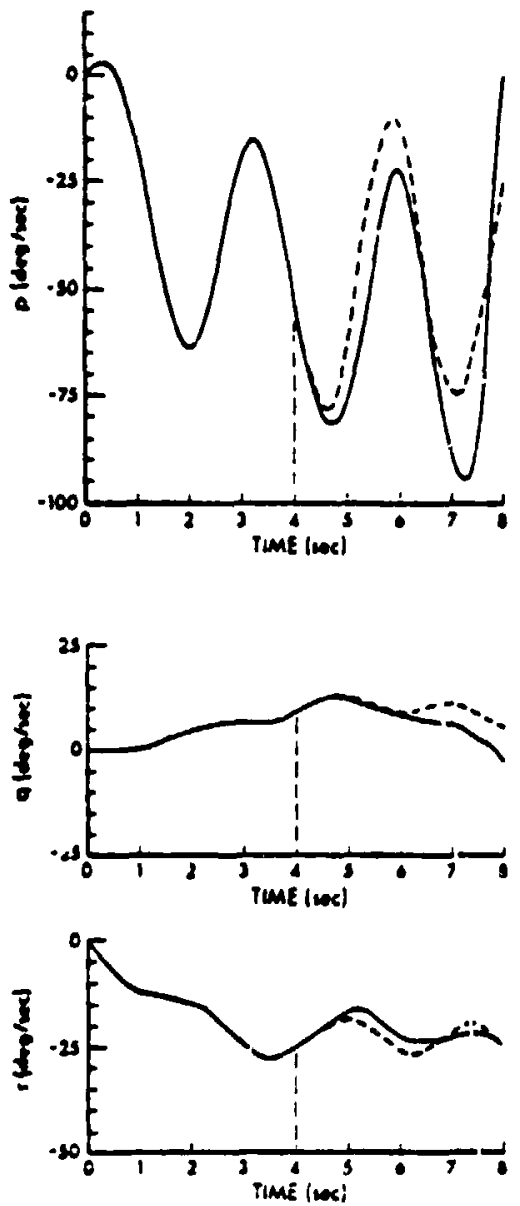

igure 2.3-5 Large Amplitude Rudder Input -- Comparison of Evolved Response With Generalized Trim Reference Foint for Linearizatiun 
trajectory. This, along with the amplitude match, supports the use of a properly linearized model for the analysis of a nonlinear vehicle along a highly dynamic trajectory. The significance of this result is put in proper perspective when it is realized that the vehicle has perform. $d$ a 360-deg roll between $t=0$ and $t=7.2 \mathrm{sec}$, and the pitch angle goes from $15 \mathrm{deg}$ to $-45 \mathrm{deg}$ from $t=0$ to $t=5 \mathrm{sec}$.

It should be noted that the nominal flight condition for linearization was found by an analytic method that does not require the solution of a nonlinear trajectory from which to estimate nominal values. The generalized trim procedure is a useful method for calculating nom $\perp$ nal flight conditions even along highly dynamic trajectories.

Summary - These comparisons presented here establish that nomisal flight conditions which satisfy the generalized trim condition produce good trajectory matches and that the corresponding linear models should provide accurate information about the nonlinear system dynamics.

\subsection{EFFECTS OF ANGULAR MOTION AND FLIGHT CONDITION ON AIRCRAFT STABILITY}

The effects of altiude and velocity variations, angle-of-attack and sideslip angle variations, and steady angular rates on aircraft stability are examined in this section using the linearized dynamic models and eigenvalue/ eigenvector analysis technique presented in Appendix A. The purpose of this aralysis is to show the effects of individual flight variables, as well as ihe combined effects of flight variables which normally are zero in "1-g" straightand-level flight. For this study, the aircraft is trimmed initially for " $1-g$ " flight at an angle of attack of 15 deg 
and at an altitude of $6100 \mathrm{~m}$. As flight variables change, the lcad factor may change accordingly; however, the primary objective of this chapter is to isolate the individual effects of each specific flight variable being examined, so all other variables are held at their initial values.

\subsubsection{Altitude and Velocity Effects}

Altitude affects the air density and, therefore, the dynamic pressure. This causes the aerodynamic forces and moments to be reduced, relative to the inertial effects, as altitude increases, as shown in Fig. 2.4-1. Higher altitude causes both the natural frequencies and damping ratios of the Dutch roll and short period modes to decrease. The roll mode also slows down as altitude increases.

Changes in velocity affect the dynamic pressure, as well as the angular rate normalization terms ( $r / 2 \mathrm{~V}$ and $\bar{c} / 2 \mathrm{~V})$ and the velocity-angular rate cross-product terms. These changes cause significant increases in Dutch ioli and short period trequencies as velocity increases (Fig. 2.4-1a). The damping ratio of the short period mode is affected only slightly by velocity changes over the range shown in Fig. 2.4-1b. The Dutch roll damping decreases as velocity decreases, so that the Dutch roll is unstable at the lowest velocities presented here. The reference aircraft's roll mode (Fig. 2.4-1c) is changed only slightly as velocity varies, contrary to the result obtained from the approximate laterallongitudinal equations discussed below.

Figure 2.4-1 indicates only small increase in spiral mode speed and phugoid frequency and damping at lower aititudes. Low velocity results in low phusoid 
- I imaginarary parts of complex
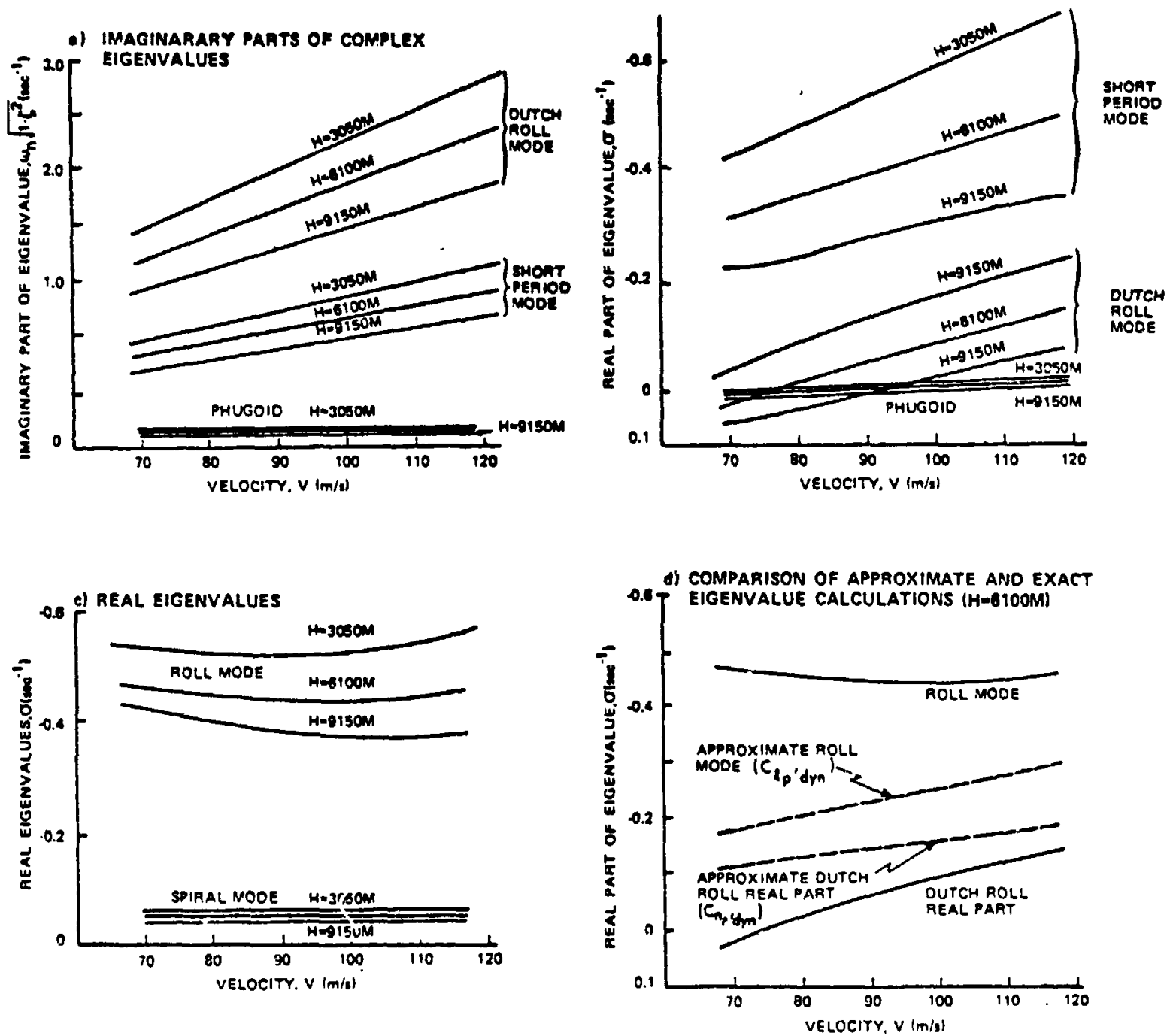

Figure 2.4-1 Altitude and Velocity Effects on Eigenvalues 
damping, so much so that the mode is unstable over a significant portion of the velocity range examined in Fig. 2.4-1.

As noted above, the lack of roll mode variation with velocity is contrary tc results obtained with accepted approximations. The expected linear change in roil mode with velocity is deduced from approximate lateral-directional equations, which can be derived by neglecting the roll angle equation (and, therefore, the spira mode), by assuming that the Dutch roll mode consists of wind-axis yawing motion, and by assuming that the roll mode consists of wind-axis roll. The approximations that result from these approximate lateraldirectional equations are

$$
\begin{aligned}
& \lambda_{\text {roll }} \cong \frac{1}{4 I_{x}} \rho_{0} v_{0} s b^{2} c_{I_{p, d y n}} \\
& \zeta \omega_{n, D R} \cong-\frac{1}{8 I_{z}} \rho_{0} v_{0} s b^{2} c_{n_{r, d y n}} \\
& \omega_{n, D R}^{2} \cong \frac{1}{2 I_{z}} \rho_{0} v_{0}^{2} s b c_{n_{B, d y n}}
\end{aligned}
$$

where $\lambda_{\text {roll }}$ is the roll mode eigenvalue, $C_{n_{\beta}, d y n}$ is defined as in $\mathrm{Eq} .(2.2-1)$ and

$$
\begin{aligned}
C_{1_{p, d y n}} & \cos \alpha_{0}^{2} C_{1_{p}}+\cos \alpha_{0} \sin \alpha_{u} C_{1_{r}} \\
& +\left(I_{x} / I_{z}\right)\left(\sin \alpha_{0} \cos \alpha_{0} C_{n_{p}}+\sin ^{2} \alpha_{0} C_{n_{r}}\right) \\
C_{n_{r, d y n}=} & \cos ^{2} \alpha_{0} C_{n_{r}}-\sin \alpha_{0} \cos \alpha_{0} C_{n_{p}} \\
& +\left(I_{z} / I_{x}\right)\left(-\sin \alpha_{0} \cos \alpha_{0} C_{1}+\sin ^{2} \alpha_{0} C_{1_{p}}\right)
\end{aligned}
$$


Equations (2.4-1) and (2.4-2) predict values for $\lambda_{\text {roll }}$ and $\zeta \omega_{n, D R}$ as shown by the dotted lines in Fig. 2.4-1d for the $6100-m$ case. The actual values, taken from Figs. 2.4-1b and $c$, are shown by solid lines on the same figure. The approximate equations do a poor job of predicting mode speed because the subject aircraft is fuselage-heavy (high $I_{z} / I_{x}$ ratio); hence, the Dutch roll contains more rolling response than is assumed when deriving the approximate equations. As can be seen from Fig. 2.4-1d, this leads to a damping interchange such that the roll mode is faster than expected and the Dutch roll mode is more poorly damped than predicted.

This examination leads to the following conclusions for the reference aircraft:

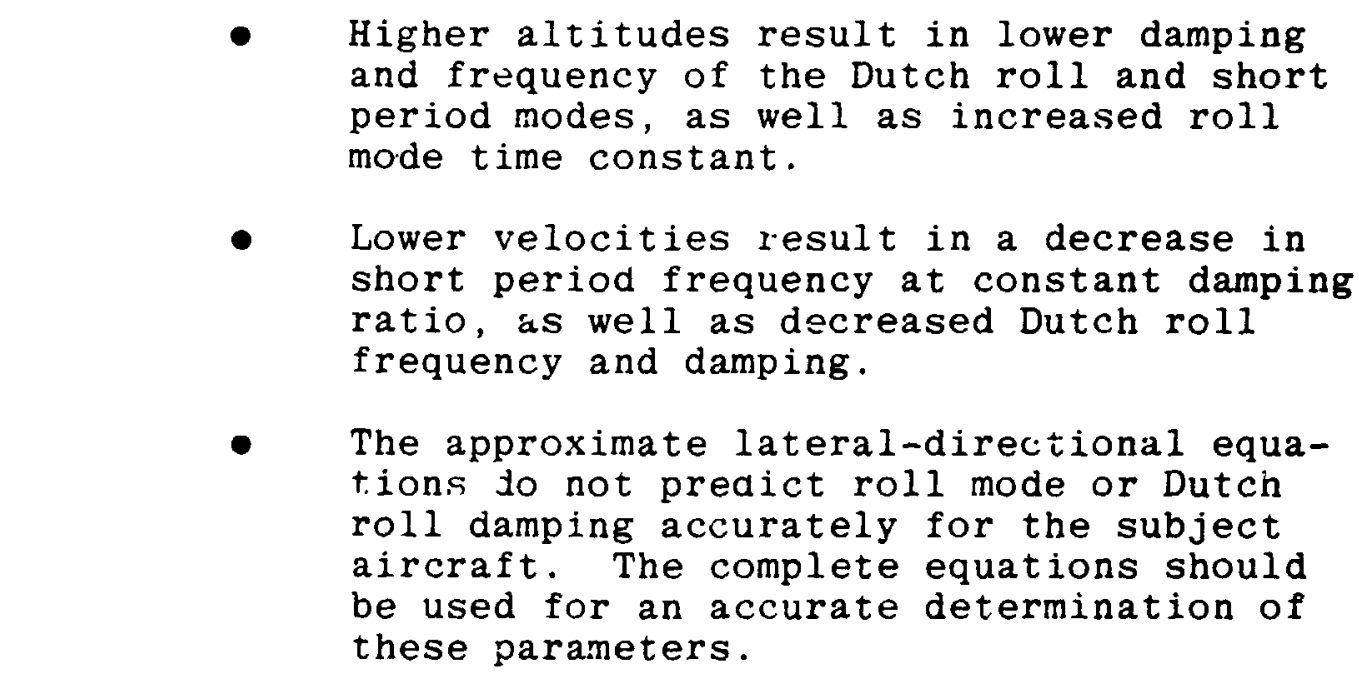

\subsubsection{Aerodynamic Angle Efiects}

The aerodynamic angles, $\alpha$ and $\beta$, specify the orientation of the vehicle relative to the velocity vector, and, to a large extent, they define the flow field around the vehicle. For this reason, the aerodynamic angles are prime determinants of the aerodynamic forces and moments. Consequently, 
significant differences in the speeds and shapes of the normal modes occur as $a_{0}$ and $B_{0}$ are varied.

Figure 2.4-2 illustrates the boundaries between stability and instability which result from these variations. These boundaries define the $\alpha_{0}$ and $\beta_{0}$ for which the real part of one or more eigenvalues migrates from negative (stable) to positive (unstable) sign (see Section A.4.1). The phugoid mode is a slow mode and is unstable at low $\alpha_{0}$. The Dutch roll mode, a fast mode, becomes unstable at high $\alpha_{0}$. The dashed line in Fig. 2.4-2 is an important boundary, indicating the transition of a relatively slowly divergent phugoid oscillation into two real roots, one of which is highly unstable. This transition line occurs at high $B_{0}-$ about 10 to $15 \mathrm{deg}$.

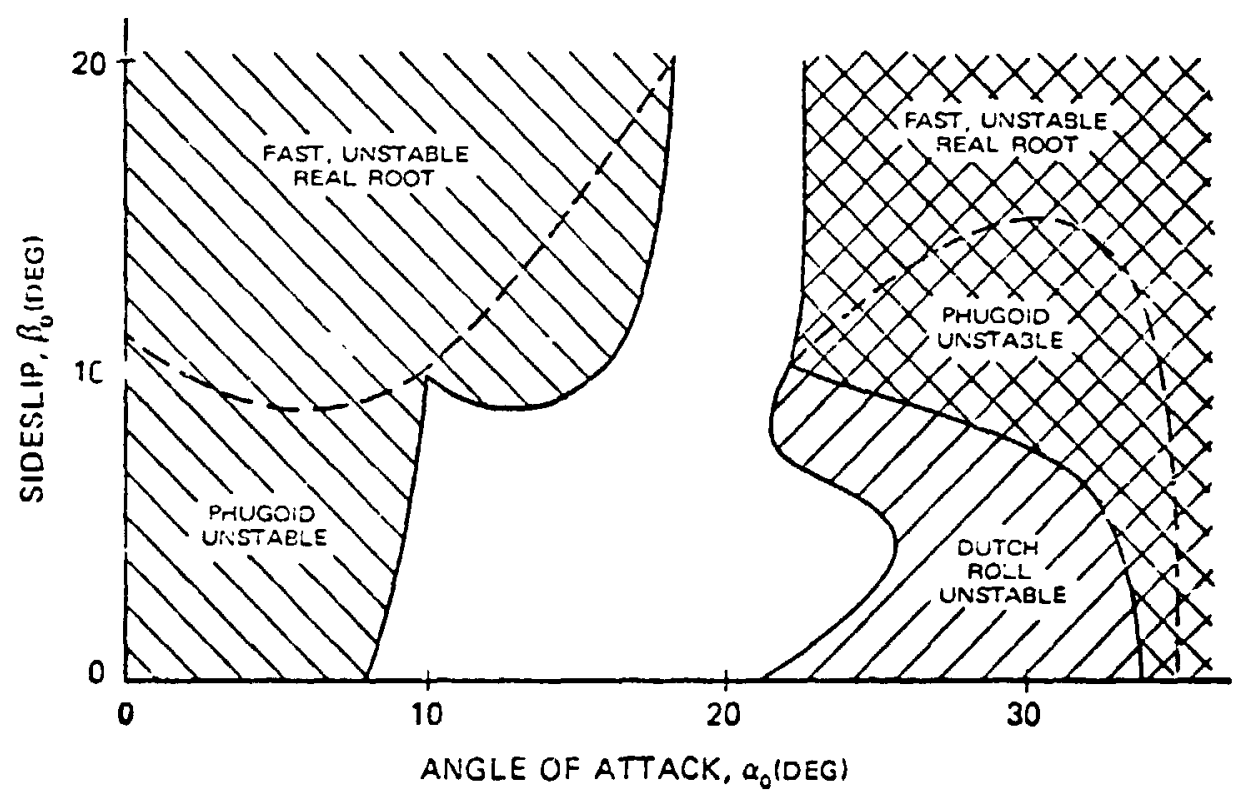

Figure 2.4-2 Effects of Aerodynamic Angles on Aircraft Stability

The shape of the Dutch roll stability boundary indicates that moderate values of nominal sideslip angle (two to five deg) stabilize the mode. This is due to lateral-longitudinal coupling; a close examination of the 
Dutch roll/short period eigenvalues indicates that, up to about five deg of sideslip, Dutch roll damping increases as short period damping decreases.

The eigenvectors of the linearized model provide information about the normal mode shapes which indicate the involvement of each state in each mode. Figure 2.4-3 illustrates some specific eigenvalue/eigenvector variations with angle of attack. Real eigenvectors, such as those associated with the roll mode, are characterized only by the relative magnitudes of each state, as the phase angles are either 0 or $180 \mathrm{deg}$. A time history of this mode would show a constant ratio between the various state amplitudes. These amplitudes would evidence exponential decays with equal time constants, given by the negative inverse of the eigenvalue. Complex eigenvectors, such as those of the Dutch roll, are characterized by the relative magnitudes of the involved states and by the phase angle between them. A time history of this oscillatory mode is generated by the projections of the eigenvectors on the real axis as the entire eigenvector set rotates with angular rate given by the imaginary part of the eigenvalue. The magnitudes decay exponentially with the time constant given by the negative inverse of the eigenvalue's real part.

Despite the large changes in eigenvalues with angle of attack, Fig. 2.4-3 shows little corresponding change in eigenvector shape. The only major changes involve the proportions of angular rates in the fast modes, and these changes are due to mode speed variations, as described above for the roll mode. The short period mode contains increased pitch rate at large $\alpha_{0}$ for this reason; in the Dutch roll eigenvector, the roll rate-to-sideslip ratio increases and decreases with the Dutch roll frequency. Overall, the Dutch 


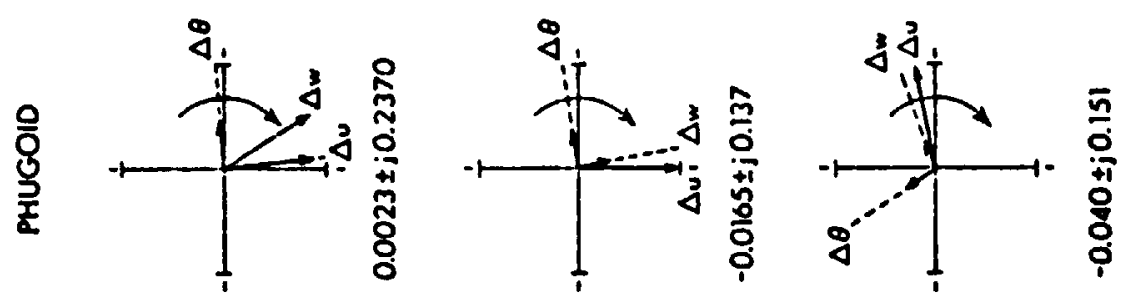

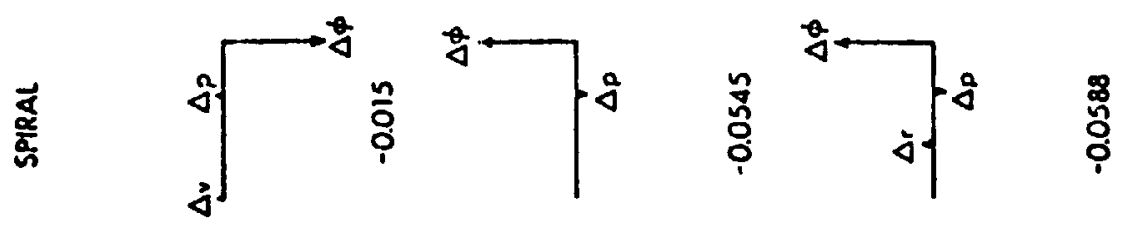

告

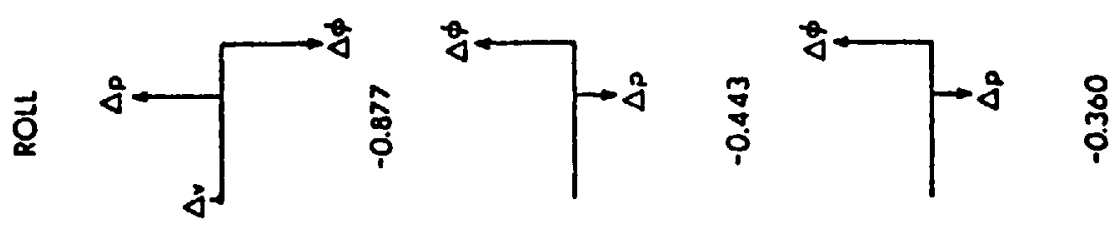

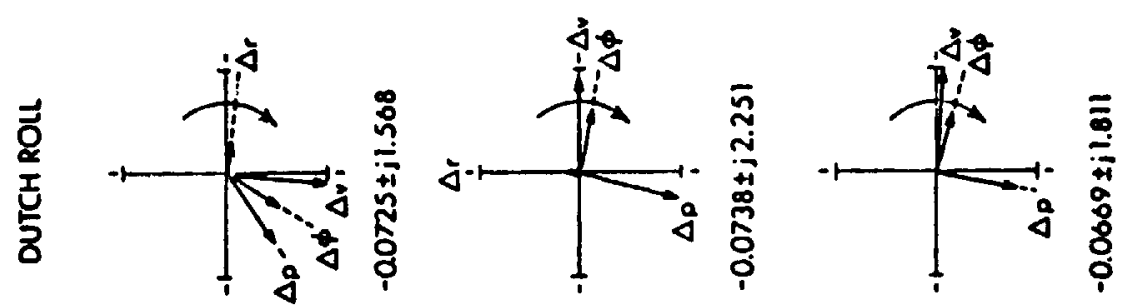

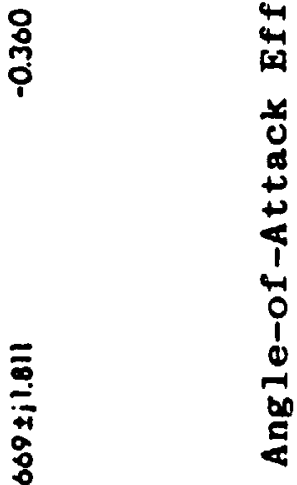
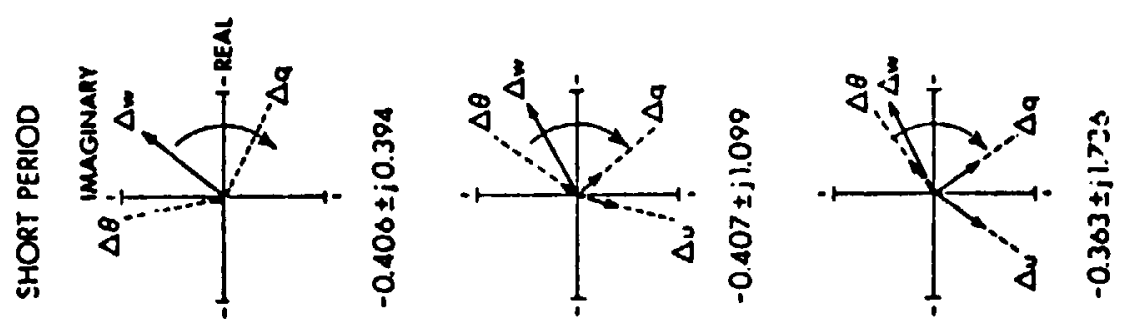

$p$
1
0
0
0
0
0
0
0

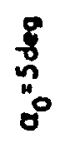

8
$\stackrel{0}{\circ}$
0
0

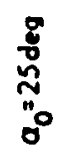


roll mode of this aircraft involves a great deal of rolling motion, underlining the low rolling inertia typical of modern fighters.

The short period eigenvector shows that this oscillation typically"involves angle-of-attack perturbations at constant velocity, as axial and normal velocity perturbations are approximately $180 \mathrm{deg}$ out of phase with each other and are related in magnitude by $\tan \alpha_{0}$. The short period mode is faster at high angle of attack, and it includes more pitch rate than at low angle of attack.

The changes in specific eigervalues and eigenvectors with sideslip angle are illustrated in Fig. 2.1-4. Laterallongitudinal coupling is quite prominent for the asymmetric flight conditions portrayed in this figure. Modes of comparaule speed couple most readily. Roll angle response is found in the phugoid mode, and pitsh angle becomes a component of the spiral mode, so that both modes involve slow roll-pitch motion. Angle of attack appears in the Dutch roll eigenvector, and a roll-sideslip combination becomes important in the short period mode, so that both modes involve an angle of attacksideslip oscillation. In both cases, $\Delta w$ and $\Delta v$ (or, equivalently, $\Delta \alpha$ and $\Delta \beta$ ) are almost $180 \mathrm{deg}$ out of phase. Note that the changes in the speeds of these modes are small and gradual as the sideslip angle is varied.

To demonstrate some of the causes of aerodynamic angle effects observed above, the aerodynamic coefficients are held constant (at the values for $\alpha_{0}=15 \mathrm{deg}$ and $B_{0}=$ 0 deg), and the body orientation with respect to the velocity vector is varied over the same range of aerodynamic angles used in Fig. 2.4-2. The results, shown in Fig. 2.4-5, differ significantly from those shown in Fig. 2.4-2. There is only a slizht sideslip effect. The Dutch roll mode, rather than 

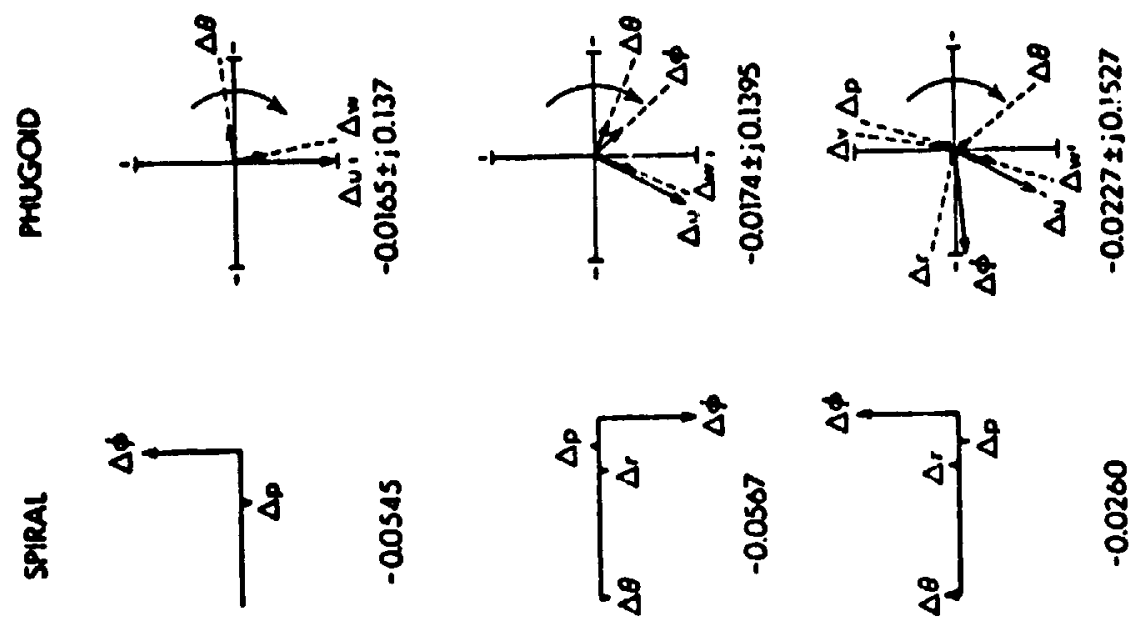

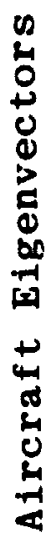
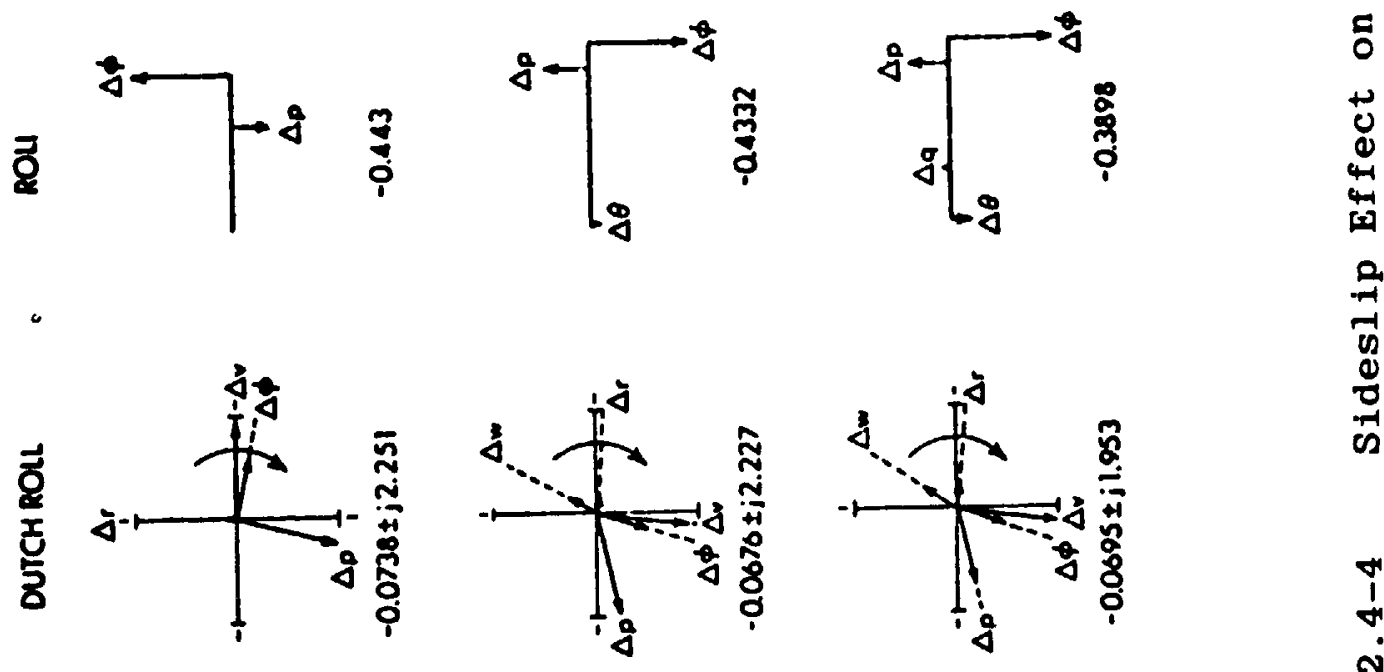

0
-1
0
0
$d$
$\infty$
$\infty$
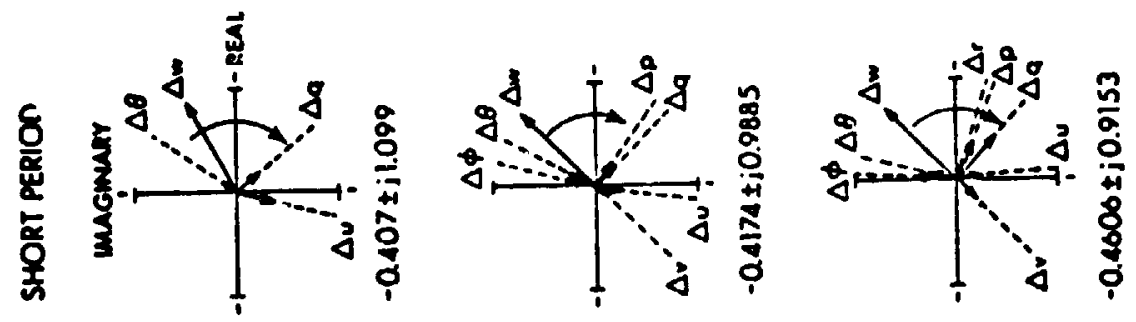

N
i

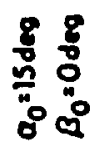

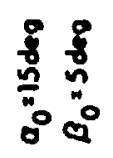

疍 


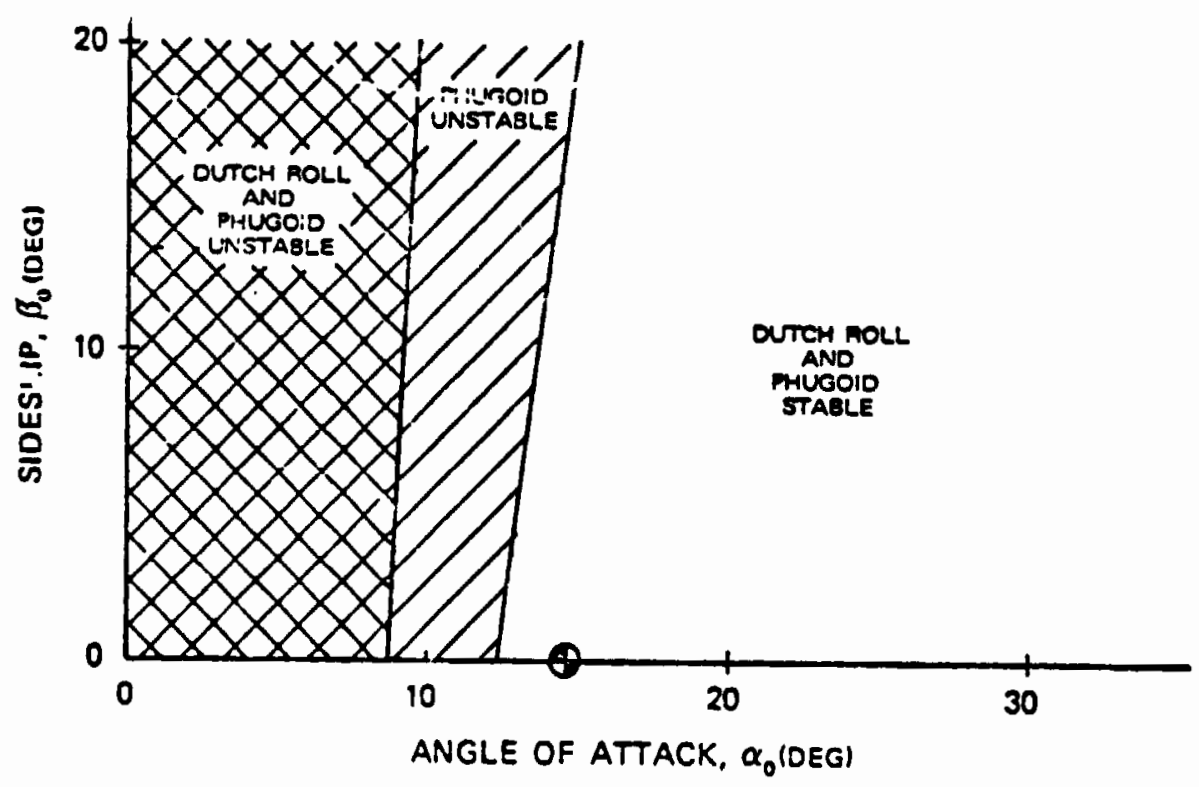

Figure 2.4-5 Effects of Body Orientation on Aircraft Stability

becoming unstable at high $\alpha_{0}$, is destabilized by lower $\alpha_{0}$. The phugoid stability boundary near $\alpha_{0}=12 \mathrm{deg}$ is roughly similar to that found in Fig. 2.4-2, indicating that the lack of phugoid stability in this area is not due to aerodynamic variations.

Figure 2.4-6 assists in the evaluation of the high angle-of-attack Dutch roll instability. This figure compares the Dutch roll eigenvalue to the departure parameter $C_{n_{B} \text {,dyn }}$ (see Section 2.2) and to $C_{n_{r}}$ and $C_{n_{r} \text {, dyn }}$, the last of which is defined in Eq. (2.4-5).

The results of Fig. 2.4-6 indicate that, at least in this case, $C_{n_{\beta} \text {, dyn }}$ is a good indicator of the Dutch roll mode's imaginary part. Neither $C_{n_{r}}$ nor $C_{n_{r} \text {, dyn }}$ provide a particularly useful indication of Dutch roll stability. This example indicates that $C_{n_{B}}$, dyn has only limited value as a departure parameter. For this aircraft, Dutch roll 


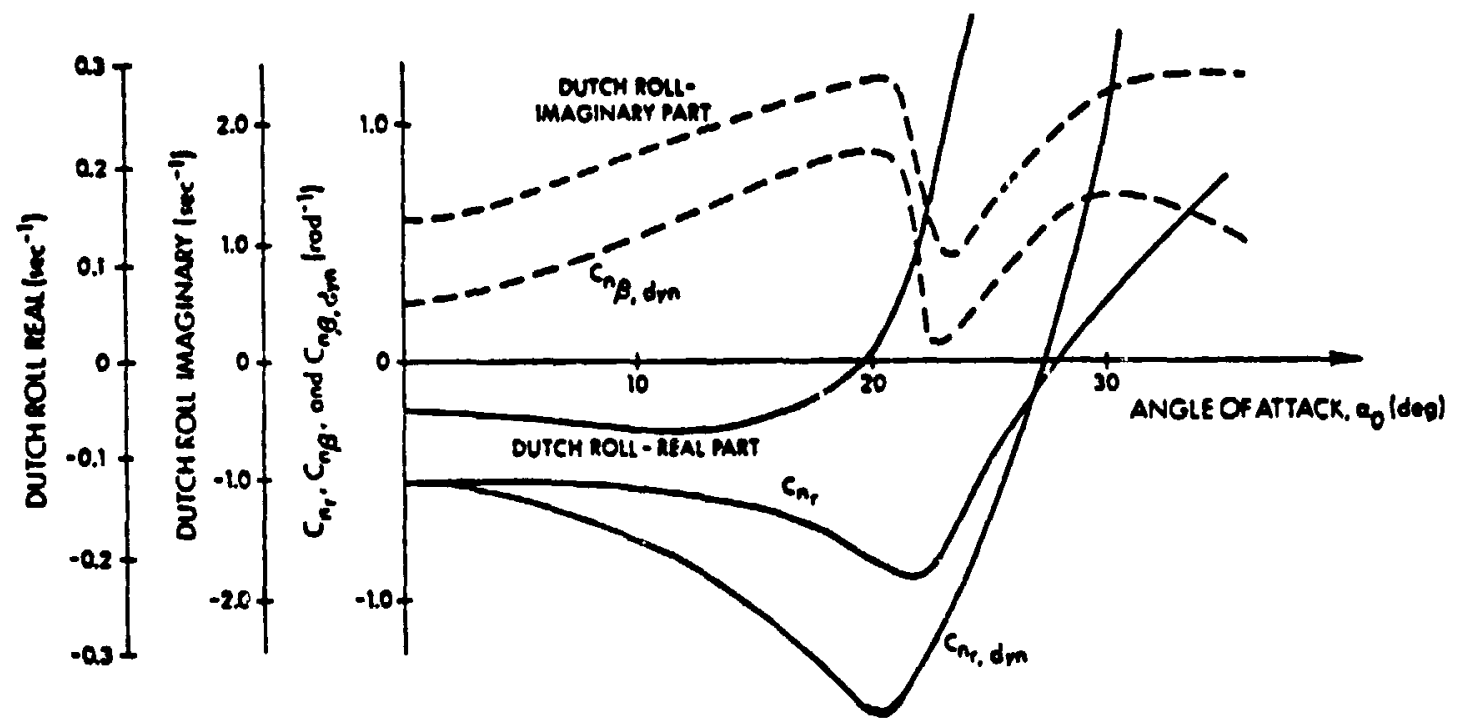

Figure 2.4-6 Variations of Directional Aerodynamic Coefficients with Angle of Attack

instability is due to negative damping, and $C_{n_{B} \text {,dyn }}$ is inadequate as a predictor of departure.

The following conclusions concerning aerodynamic angle effects on stability of the reference aircraft can be made:

- The Dutch roll mode becomes unstable due to negative damping at high $\alpha_{0}$. This is caused by changes in the aerodynamics as $\alpha_{0}$ increases.

- Mean angle of attack variations have significant effect on the eigenvalues, but mode shape (eigenvector) changes are small relative to other effects.

- Mean sideslip angle introduces laterallongitudinal coupling; therefore it has a large effect on the mode shapes (eigenvectors), without causing large changes in the elgenvalues. This laterallongitudinal coupling primarily occurs between modes of similar speed and can lead to a transfer of damping, as in the situation where small sideslip angles 
stabilize the Dutch roll mode at the expense of short periud damping.

- The parameter $C_{n_{B}, d y n}$ gives a good indication of Dutcin roll frequency, but it is not useful as a departure parameter for the subjest aircraft.

\subsubsection{Angular Rate Effects}

Non-zero nominal argular rates have two effects on the linearized aircraft uynamics. The first, an aerodynamic effect, results in a change in the nominal forces and muments due to the steady angular rates. The second is dynamic, and it is due to the cross product of angular rate with velocity (in the force equations) and with angular momentum (in the moment equations). The specific terms involved (for $I_{x z}=0$ ) are given in Table 2.4-1. A close examination reveals that mean pitch angular rate, $\mathrm{q}_{0}$, enters both the lateral and longitudinal equations but does not affect lateral-longitudinal coupling terms. Mean roll and yaw rates, $p_{0}$ and $r_{0}$, enter as lateral-longitudinal coupling terms. Steady rollrate capability of most high-performance aircraft is much higher than pitch-or yaw-rate capability, so roll-rate effects are especially important.

Stability boundaries as functions of pitch rate and yaw rate are illustrated in Fig. 2.4-7. The destabilizing influence of $q_{0}$ is the major effect, and it has an especially severe effect on the Dutch roll mode. Yaw rate has a mild stabilizing effect on the Dutch roll and spiral modes. This is due partially to lateral-longitudinal coupling, because short period and phugoid damping decrease as Dutch roll damping increases. 
TABLE 2. 4-1

DYNAMIC EFFECTS OF STEADY ANGULAR RATE

\begin{tabular}{|c|c|c|}
\hline Angular Rate & Multiplied By & Enters Term \\
\hline$p_{0}$ & $\left(I_{z}-I_{x}\right) / I_{y}$ & $\partial \dot{q} / \partial r$ \\
$p_{0}$ & -1 & $\partial \dot{w} / \partial v$ \\
$p_{0}$ & 1 & $\partial \dot{v} / \partial w$ \\
$p_{0}$ & $\left(I_{x}-I_{y}\right) / I_{z}$ & $\partial \dot{r} / \partial q$ \\
$q_{0}$ & -1 & $\partial \dot{u} / \partial w$ \\
$q_{0}$ & 1 & $\partial \dot{w} / \partial u$ \\
$q_{0}$ & $\left(I_{x}-I_{y}\right) / I_{z}$ & $\partial \dot{r} / \partial p$ \\
$q_{0}$ & $\left(I_{y}-I_{z}\right) / I_{x}$ & $\partial \dot{p} / \partial r$ \\
$r_{0}$ & 1 & $\partial \dot{u} / \partial v$ \\
$r_{0}$ & $\left(I_{z}-I_{x}\right) / I_{y}$ & $\partial \dot{q} / \partial p$ \\
$r_{0}$ & -1 & $\partial \dot{v} / \partial u$ \\
$r_{0}$ & $\left(I_{y}-I_{z}\right) / I_{x}$ & $\partial \dot{p} / \partial q$ \\
\hline
\end{tabular}

ZD DUTCH ROLL STABLE

$\triangle D$ PHUGOID STABLE

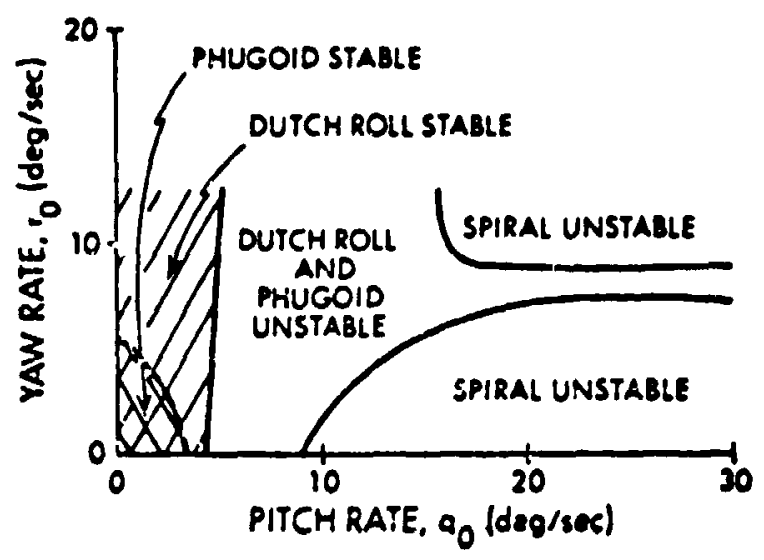

Figure 2.4-7 Yaw-Rate/Pitch-Rate Effects $\left(\alpha_{0}=15 \mathrm{deg}\right)$ 
$\int_{\frac{8}{2}}^{8}$

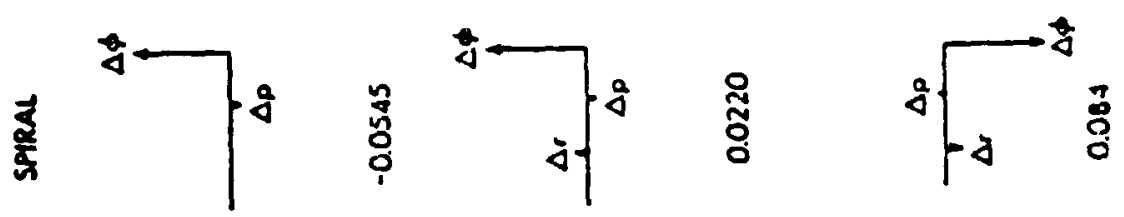

告
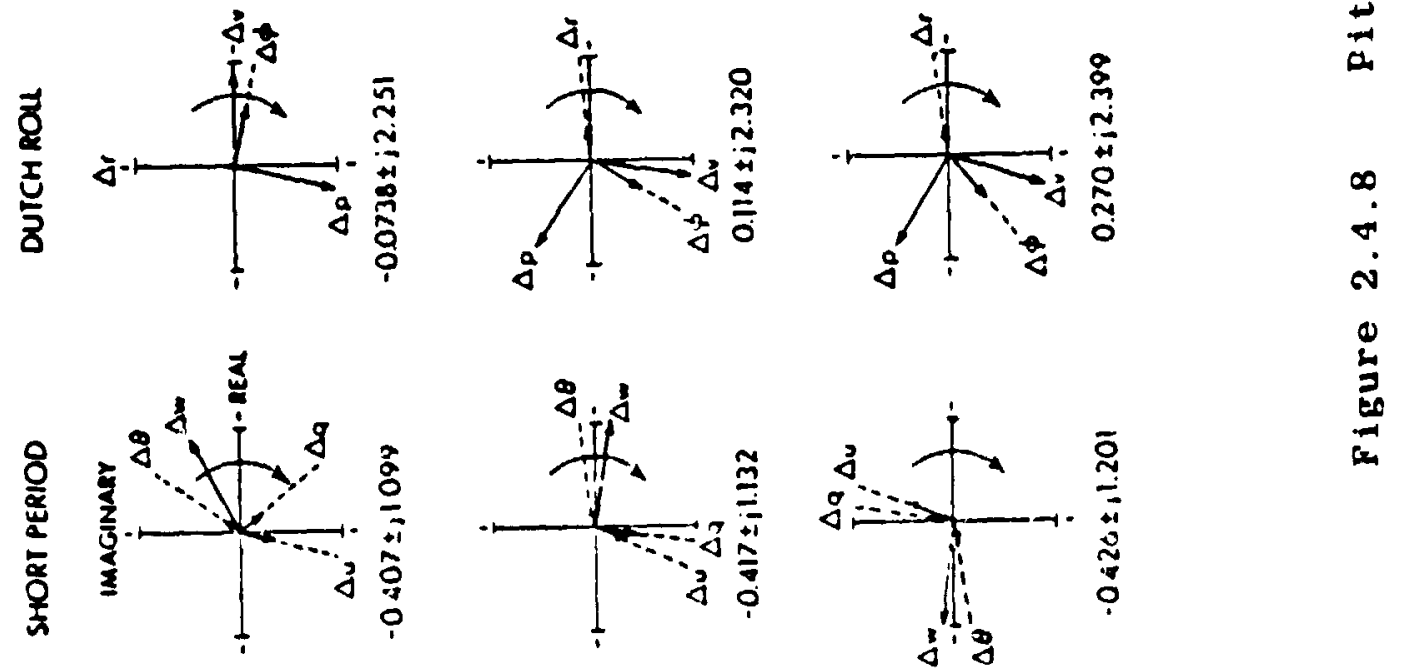

产

苞

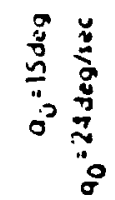


The eigenvector changes that accompany increases in $\mathrm{q}_{0}$ are shown in Fig. 2.4-8. Steady pitch rate does not introduce lateral-longitudinal coupling, but soine changes in mode shapes appear in the roll mode and in the separation of the complex phugoid mode into two real roots. Both frequencies and aamping ratios of the Dutch roll and short period modes change, but changes in the mode shape are minor.

Steady roll rate is important because fighter aircraft are capable of hic:. $\mathrm{p}_{0}$, and air combat maneuvers often include such motions. For the aircraft to roll with constant aerodynamic angles, the roll rate must occur about the wind $x$-axis (which is the same as the stability $x$-axis for constant nominal aerodynamic angles). Sideslip variations also are considered, since piloting error can easily result in non-zero $B_{0}$ during a rolling maneuver. Both positive and negative $p_{0}$ are considered, to account for roll "into" or "out of" the sideslip.

The stability boundaries that result from combined ro:.' rate and sideslip are shown in Fig. 2.4-9. These boundaries indicate that $p_{0}$ has only a small effect on the fast modes, primarily the Dutch roll mode. The combination of $\mathrm{p}_{0}$ and small values of ${ }^{B}{ }_{C}$ of opposite sign serves to destabilize the Dutch roll mode. Roll rate destabilizes the phugoid mode in general, but there is a combination of ${ }^{B_{0}}$ and $p_{0}$ that maintains phugoid stability. High $\beta_{0}$ results in a fast divergence for all values of $\mathrm{o}_{0}$ tested.

Eigenvector variations due to steady rolling are illustratzd in Fig. 2.4-10. Eigenvalue changes are significant, considering the angular rates involved. The mode shapes also change, so that lateral-longitudinal coupling is important. Large roll-rate/sidesip perturbations in 


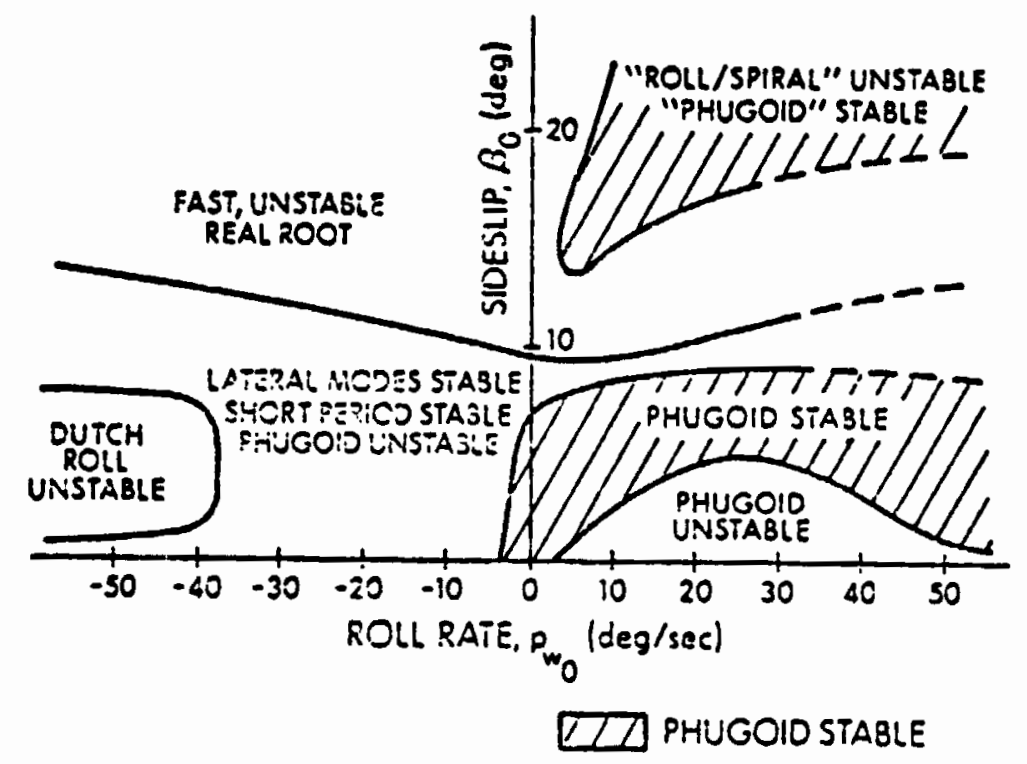

Figure 2.4-9 Stability Boundaries for Sideslip/ Roll-Rate Variations $\left(\alpha_{0}=15\right.$ deg $)$

the short period mode and large angle of attack perturbations in Dutch roll mode are examples of this coupling.

Conclusions about steady angular rate effects are

as follows :

Mean yaw rate and roll rate cause lateral-longitudinal coupling and therefore change the mode shapes significantly. Roll rate is by far the more significant because of the large values it can exhibit.

Mean pitch rate changes the speeds of the normal modes vithout affecting their shapes significantly. Even low values of $q_{0}$ (about $5 \mathrm{deg} / \mathrm{sec}$ ) can cause the Dutch roll mode to be unstable. 

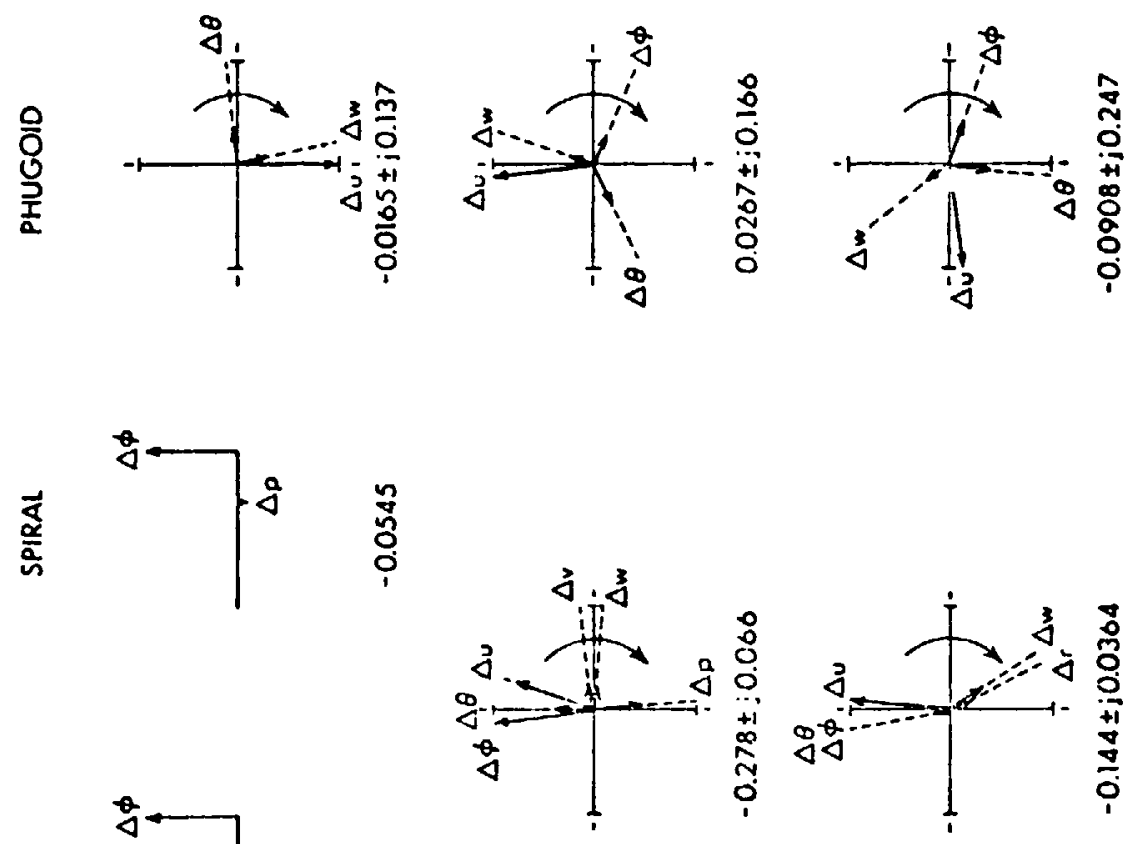

$n$
+
0
01
$4=1$
01

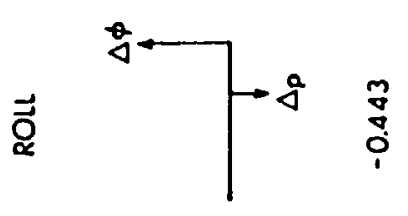

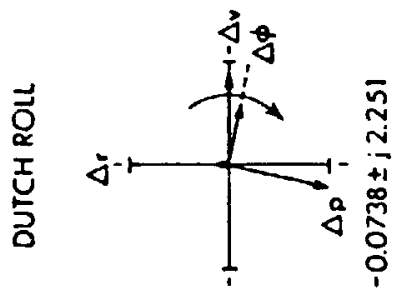

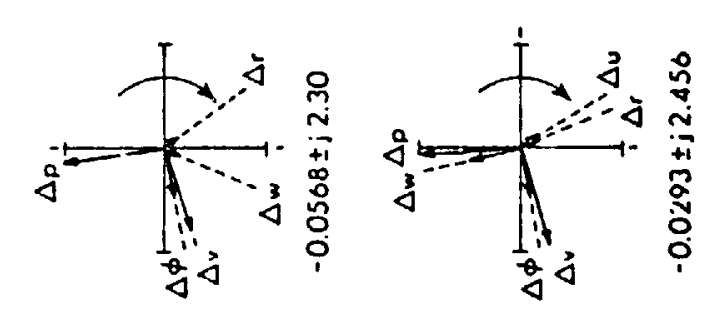

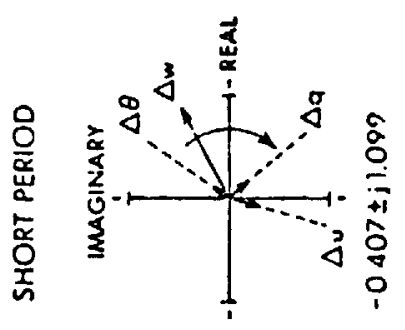
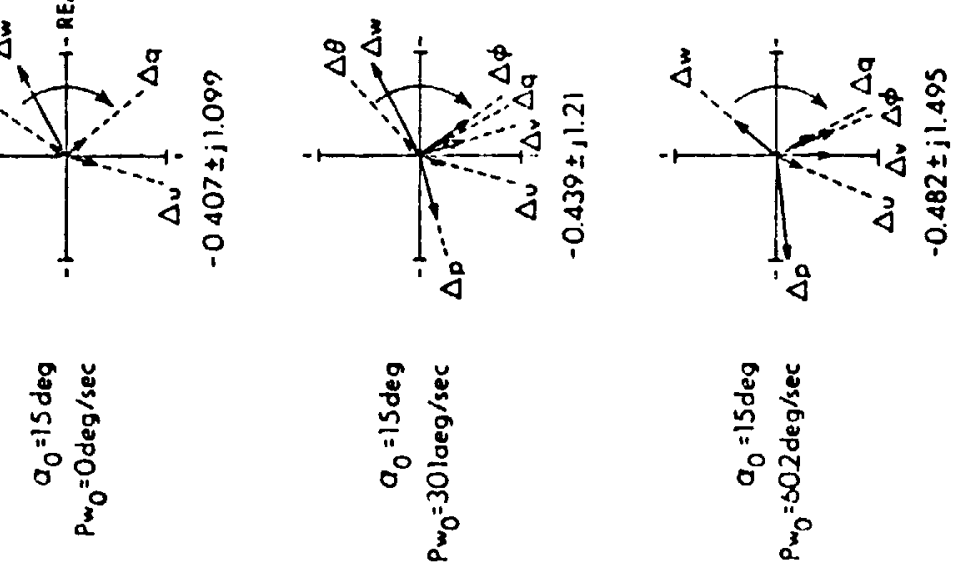


\subsection{EFFECTS OF ANGULAR MOTION AND FLIGHT CONDITION ON AIRCRAFT CONTROL}

The transfer function provides a primary measure of the quality of aircraft control, as it is the Laplace transform of the ratio between a specific output and a specific input (Appendix $A$ ). The transfer function gain, $K_{F}$, is the stady-state value of the transfer function after all transients damp out, assuming that all transients are stable. The transfer function gain, $\mathbb{K}_{I}$, is (for most aircraft), the initial state rate response to a transfer function's control step. The poles of the transfer function are the eigenvalues of the unforced system, as described in Appendix A.

The zeros affect the magnitudes of excitation of the normal modes, which are related to the distance between the zeros and the appropriate eigenvalues in the $s$ plane. In the limiting case, a zero and pole in the same location cancel, and the corresponding mode does not appear in that response. Zeros located in the right half-plane are called nonminimum-phase zeros (due to their effects on the phaseshift of sinusoidal inputs), and they have major impact on the aircraft's transient response and on controller design. For example, an undesirable reversal in the initial response is caused by such zeros, as illustrated in Fig. 2.5-1.

The nonminimum-phase type of response is undesirable because it makes closed-loop control difficult. The pilot can be misled by this type of response, as the magnitude and sign of the motion are uncertain. Addition of a high-gain feedback loop around a transfer function that exhibits nonminimum-phase properties can result in instability of the 


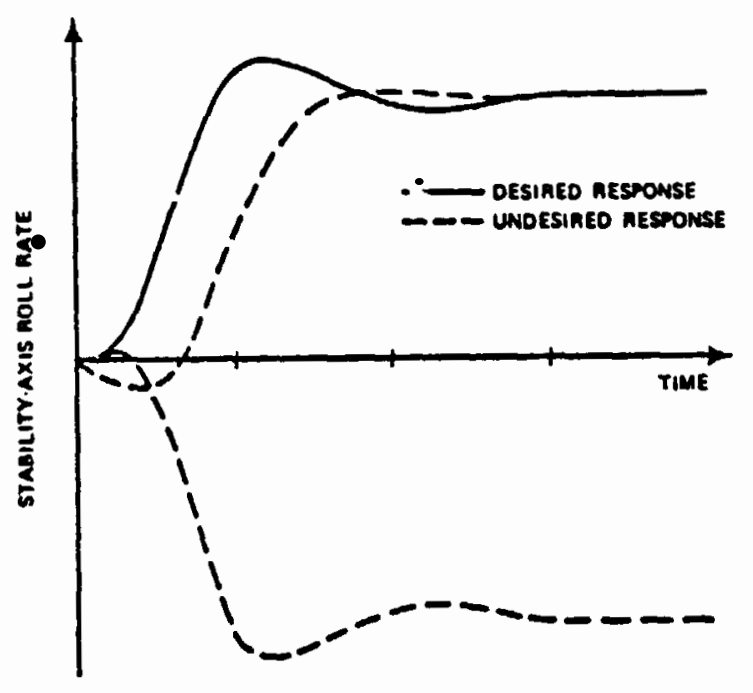

Figure 2.5-1 Typical Step Response Forms

closed-loop system (Ref. 9). Finally, this type of response can make it impossible to implement some simple forms of adaptive control, as they can suffer from instability for an analogous reason (Ref. 57).

\subsubsection{Velocity and Aerodynamic Angle Effects}

As has been observed previously (Section 2.4.1), velocity changes the dynamic pressure, which affects the control effectiveness. Table 2.5-1 illustrates some typical values of the transfer function gain, $K_{F}$, at different speeds, and the variation is as expected. Altitude also affects dynamic pressure in that increasing altitude decreases atmospheric density; hence, dynamic pressure decreases. 
TABLE 2.5-1

VELOCITY EFFECTS ON TRANSFER FUNCTION GAIN, $\mathbf{K}_{\mathbf{F}}$

\begin{tabular}{|c|c|c|c|}
\hline Velocity & $\Delta w / \Delta \delta_{h}$ & $\Delta p / \Delta \delta_{a}$ & $\Delta r / \Delta \delta_{2}$ \\
\hline \hline $70 \mathrm{~m} / \mathrm{s}$ & -3.88 & -0.50 & -4.04 \\
$94 \mathrm{~m} / \mathrm{s}$ & -5.17 & -0.66 & -5.39 \\
$117 \mathrm{~m} / \mathrm{s}$ & -6.47 & -0.83 & -6.74 \\
\hline
\end{tabular}

Aerodynamic angle variations can cause large changes in the system eigenvalues and can be expected to have significant effects on the numerator of the transfer function as well. Table 2.5-2 illustrates variations in $\mathrm{K}_{I}$ and $\mathbf{B}_{F}$ as $\alpha_{0}$ and $B_{0}$ vary. (See Section A.4.3 for the definition of $K_{I} \cdot$ ) The invariability of $K_{I}$ with sideslip indicates that the control effectiveness does not depend on sideslip. This is a function of the aerodynamic data used here (Appendix $B$ ), as the data does not model the effects of $B_{0}$ on control effectiveness. The steady-state gain, $K_{F}$, shows a large dependence on sideslip because this gain depends on the pole and zero locations, which themselves vary with $B_{0}$.

The sign changes in $K_{F}$ (at small $\alpha_{0}$ ) as $\beta_{0}$ varies are due to changes in the number of unstajle poles and nonminimum-phase zeros. From Eq. (2.5-6), it can be seen that such a change results in a $K_{F}$ sign change if the sign of $K_{I}$ remains the same.

One of the major effects of angle-of-attack variations (best seen in the $s_{I}$ gain of the $\Delta p / \Delta \delta_{a}$ transfer 
TABLE 2.5-2

AERODYNAMIC ANGLE EFFECTS ON TRANSFER FUNCTION GAINS $\left(\mathrm{V}_{0}=94 \mathrm{~m} / \mathrm{s}\right)$

\begin{tabular}{|c|c|c|c|c|c|c|c|c|c|c|c|}
\hline \multirow[b]{2}{*}{$a($ deg $)$} & \multirow[b]{2}{*}{$A(\operatorname{deg})$} & \multicolumn{7}{|c|}{$k_{F}$} & \multicolumn{3}{|c|}{$x_{I}$} \\
\hline & & $\Delta w / \Delta \varepsilon_{b}$ & $\Delta v / \Delta \delta_{3}$ & $\Delta r / \Delta \delta_{2}$ & $\Delta P / \Delta \varepsilon_{2}$ & $\Delta v / \Delta t_{r}$ & $\Delta r / \Delta \theta_{r}$ & $\Delta \mathrm{p} / \Delta \sigma_{\mathrm{g}}$ & $\Delta w / \Delta \delta_{h}$ & $\Delta p / \Delta \delta_{a}$ & $\Delta r / \Delta \delta_{r}$ \\
\hline 5.0 & 0.0 & -1.04 & 2.46 & 18.39 & -4.91 & -0.85 & -7.11 & 1.90 & -0.10 & 2.88 & -0.53 \\
\hline 5.0 & 10.0 & 0.33 & -3.86 & -32.02 & B.64 & 0.37 & 3.04 & -0.82 & -0.10 & 2.98 & -0.53 \\
\hline 15.0 & 0.0 & -1.58 & 0.41 & 2.47 & -0.66 & -0.89 & -5.39 & 1.44 & -0.14 & 1.71 & -0.57 \\
\hline 15.0 & 10.0 & -2.37 & 1.43 & 9.88 & -2.64 & -1.71 & -11.85 & 3.27 & -0.14 & 1.71 & -0.57 \\
\hline 25.0 & 0.0 & -0.84 & 0.03 & 0.49 & -013 & -0.27 & -4.29 & 1.15 & -0.11 & -0.001 & -0.44 \\
\hline 25.0 & 10.0 & -2.50 & 0.02 & 0.42 & -0.11 & -0.05 & -3.83 & 1.02 & -0.11 & -0.001 & -0.44 \\
\hline
\end{tabular}

function) is the loss of aileron roll control at high $\alpha_{0}$. This loss of aileron roll effectiveness, combined with the continued effectiveness of the rudder for roll and yaw control, leads to the conclusion that this aircraft is rolled more effectively with the rudder at high angles of attack.

\section{An examination of the transfer function zeros}

(Table 2.5-3) indicates that nonminimum-phase zeros are quite prevalent, although often accompanied by right-halfplane poles, i.e., they often occur in unstable systems. Right-half-plane zeros are important in control system design because closed-loop poles of a system with a simple loop closure migrate from the open-loop poles to the zeros as the loop gain is increased. Therefore, in a system with right-half-plane zeros, too high a gain may result in an unstable closed-loop system.

When $B_{0}$ is not zero, there is mode coupling, and a control input excites all modes. This is indicated by Table 2.5-4, which presents the poles and zeros of three transfer functions at a flight condition where $B_{n}$ is non- 
TABLE 2.5-3

EFFECTS OF ANGLE OF ATTACK ON TRANSFER FUNCTION ZEROS

\begin{tabular}{|c|c|c|c|c|c|}
\hline $\begin{array}{l}\text { Tranefer } \\
\text { runcetion }\end{array}$ & $a_{0}(d e g)$ & & & Zaros & \\
\hline$\Delta \pi / \Delta \delta_{b}$ & $\begin{array}{r}5 \\
15 \\
25\end{array}$ & $\begin{array}{r}0.0084 \\
-0.0025 \\
-0.0281\end{array}$ & $\begin{array}{l} \pm j \\
\pm j \\
\pm j\end{array}$ & $\begin{array}{l}0.0824 \\
0.1167 \\
0.1500\end{array}$ & $\begin{array}{l}-25.89 \\
-21.07 \\
-25.56\end{array}$ \\
\hline$\Delta v / \Delta d_{\mathbf{z}}$ & $\begin{array}{r}5 \\
15 \\
25\end{array}$ & $\begin{array}{l}-0.168 \\
-0.137 \\
-0.164\end{array}$ & & $\begin{array}{r}-1.465 \\
-0.512 \\
0.178\end{array}$ & $\begin{array}{c}86.43 \\
1325.0 \\
133.7 *\end{array}$ \\
\hline$\Delta r / \Delta \delta_{2}$ & $\begin{array}{r}5 \\
15 \\
25\end{array}$ & $\begin{array}{l}0.350 \\
2.06 \\
0.036\end{array}$ & $\begin{array}{l} \pm j \\
\pm j \\
\pm j\end{array}$ & $\begin{array}{l}2.914 \\
9.63 \\
1.725\end{array}$ & $\begin{array}{l}-1.08 \\
-0.385 \\
-0.237\end{array}$ \\
\hline$\Delta p / \Delta \theta_{a}$ & $\begin{array}{r}5 \\
15 \\
25\end{array}$ & $\begin{array}{l}-0.141 \\
-0.152 \\
-13.67\end{array}$ & $\begin{array}{l} \pm J \\
\pm 1\end{array}$ & $\begin{array}{r}1.399 \\
1.295 \\
22.49\end{array}$ & $\begin{array}{l}0.0270 \\
0.0278 \\
0.0300\end{array}$ \\
\hline$\Delta v_{j}^{\prime} \Delta \sigma_{r}$ & $\begin{array}{r}5 \\
15 \\
25\end{array}$ & $\begin{array}{l}0.0198 \\
0.0958 \\
0.0795\end{array}$ & & $\begin{array}{l}-0.840 \\
-0.393 \\
-0.188\end{array}$ & $\begin{array}{l}-40.97 \\
-45.74 \\
-41.70\end{array}$ \\
\hline$\Delta r / \Delta \delta_{r}$ & $\begin{array}{r}5 \\
15 \\
25\end{array}$ & $\begin{array}{r}0.036 \\
-0.025 \\
0.023\end{array}$ & $\begin{array}{l} \pm j \\
\geq j \\
\pm j\end{array}$ & $\begin{array}{l}0.686 \\
1.720 \\
1.694\end{array}$ & $\begin{array}{l}-0.916 \\
-0.391 \\
-0.238\end{array}$ \\
\hline$\Delta p /{ }^{1}{ }_{F}$ & $\begin{array}{r}5 \\
15 \\
25\end{array}$ & $\begin{array}{l}0.027 \\
0.028 \\
0.030\end{array}$ & & $\begin{array}{l}-1.80 \\
-2.98 \\
-2.66\end{array}$ & $\begin{array}{l}1.79 \\
3.34 \\
3.05\end{array}$ \\
\hline
\end{tabular}

-Accompaled by right-half-plane poles

TABLE 2.5-4

POLE-ZERO COMPARISON AT $\alpha_{0}=15 \mathrm{DEG}, \beta_{0}=10 \mathrm{DEG}$

\begin{tabular}{|c|c|c|c|c|}
\hline \multicolumn{5}{|c|}{ Poles } \\
\hline Short Parlod & Dutcb 8021 & Ro11 & Splral & Phucold \\
\hline$-0.161 \geq 90.925$ & $-0.0605 \leq 51.953$ & -0.390 & -0.026 & $-0.0227=j 0.153$ \\
\hline
\end{tabular}

\begin{tabular}{|c|c|c|c|c|}
\hline \multicolumn{5}{|c|}{ Zeros of $\Delta \nabla / \Delta \delta_{h}$} \\
\hline-20.75 & $-0.0928 \pm 1 \quad 2.032$ & -0.435 & -0.033 & $.0 .0009=10.134$ \\
\hline \multicolumn{5}{|c|}{ Zeroe of $\Delta 5 / \Delta \sigma^{\circ}$} \\
\hline$-0.503 \pm j 1.001$ & $0.153 \pm j 1.301$ & -0.382 & & $-0.0210 \geq 10.147$ \\
\hline \multicolumn{5}{|c|}{ Zeros of $\triangle P / \Delta C_{2}$} \\
\hline$-0.440 \pm 1 \quad 1.181$ & 251.272 & & 0.028 & $-0.0204 \geq 10.143$ \\
\hline
\end{tabular}


zero. Note that the lateral mode poles are not canceled in $\Delta w / \Delta \delta_{h}$ and that the longitudinal mode poles are not canceled in the $\Delta \mathrm{r} / \Delta \delta_{\mathrm{r}}$ and $\Delta \mathrm{p} / \Delta \delta_{\mathrm{a}}$ transfer functions.

The effects of velocity and aerodynamic angles on control of the example aircraft can be summarized as follows:

- Lower velocities lead to decreased control effectiveness, as demonstrated by transfer function gains.

- Non-zero Bo does not affect $K_{I}$, but does change the poles and zeros so that all modes are excited.

- Mean angle of attack leads to significant changes in control effectiveness, so much so that the rudder is more efficient than the aileron for producing roll at high angles of attack.

- Nonminimum-phase zeros are prevalent in the aircraft transfer functions at increased angle of attack.

\subsubsection{Angular Rate Effects}

Although no explicit effects of nominal angular rates on the control effectiveness are included in the specific aerodynamic data used here, angular rates cause significant changes in the transfer functions due to pole and zero shifts. This is apparent in Table 2.5-5, which shows changes in transfer function gains due to nominal pitch rate. The initial value of the transfer function, $\mathrm{K}_{I}$, does not vary with $q_{0}$ because $K_{I}$ depends only on the control effectiveness. The steady-state gain, $K_{F}$, does vary with $\mathrm{q}_{0}$ because of the pole and zero variations. As above, sign variations in $K_{F}$ indicate the appearance of unequal numbers of nonminimum-phase zeros and right half-plane poles. 
TABLE 2.5-5

EFFECTS OF PITCH RATE ON TRANSFER FUNCTION GAINS ( $u_{0}=15 \mathrm{deg}$ )

\begin{tabular}{|c|c|c|c|c|c|c|c|c|c|c|}
\hline \multirow[b]{2}{*}{$q(d e g / s e c)$} & \multicolumn{7}{|c|}{$K_{F}$} & \multicolumn{3}{|c|}{$x_{I}$} \\
\hline & $\Delta w / \Delta s_{h}$ & $\Delta v / \Delta \delta_{a}$ & $\Delta r / C \delta_{a}$ & $\Delta p / \Delta \delta_{a}$ & $\Delta v / \Delta \delta_{r}$ & $\Delta r / \Delta \delta_{r}$ & $\triangle P / \triangle S_{F}$ & $\Delta w / \Delta \delta_{h}$ & $\angle r / \Delta \delta_{r}$ & $\Delta \mathrm{p} / \Delta \delta_{\mathrm{a}}$ \\
\hline 0 & -1.38 & 0.42 & 2.47 & -0.66 & -0.89 & -5.39 & 1.44 & -0.14 & -0.58 & 1.71 \\
\hline 12 & -5.67 & -0.02 & -2.61 & 2.44 & 0.04 & 5.68 & -5.32 & $-0.1 i$ & -0.57 & 2.71 \\
\hline 24 & -2.51 & 0.17 & -0.39 & 0.72 & -0.37 & 0.84 & 1.56 & -0.14 & -0.57 & 1.72 \\
\hline
\end{tabular}

Mean wind-axis roll rate, like $\beta_{0}$, has no effect on control power but does change the mode shapes signifjcantly. The steady-state transfer function gain varies with $p_{0}$ as shown in Table 2.5-6. This variation is fairly smooth, compared to the effects of $q_{0}$, and the only sign changes are in the roll-rate transfer functions. Because non-zero roll rate creates lateral-longitudinal coupling, any control displacement excites all of the normal modes.

TABLE $2 \cdot 5-6$

EFFECTS OF RCLL RATE ON TRANSFER FUNCTION GAIN, $K_{F}\left(\alpha_{0}=15 \mathrm{deg}\right)$

\begin{tabular}{|c|c|c|c|c|c|c|c|}
\hline $\mathrm{P}_{\mathrm{T}}(\mathrm{deg} / \mathrm{sec})$ & $\Delta \mathrm{w} / \Delta \delta_{\mathrm{b}}$ & $\Delta \mathrm{v} / \Delta \delta_{\mathrm{a}}$ & $\Delta \mathrm{r} / \Delta \delta_{\mathrm{a}}$ & $\Delta \mathrm{p} / \Delta \delta_{2}$ & $\Delta \mathrm{v} / \Delta \delta_{\mathrm{r}}$ & $\Delta \mathrm{r} / \Delta \delta_{\mathrm{r}}$ & $\Delta \mathrm{p} / \Delta \delta_{\mathrm{r}}$ \\
\hline 0 & -1.58 & 0.41 & 2.47 & -0.65 & -0.89 & -5.39 & 1.44 \\
15 & -1.64 & 0.24 & 0.69 & -0.11 & -0.52 & -1.49 & 0.23 \\
30 & -1.40 & 0.22 & 0.32 & 0.06 & -0.51 & -0.73 & -0.13 \\
45 & -1.25 & 0.22 & 0.18 & 0.10 & -0.62 & -0.51 & -0.29 \\
60 & -1.23 & 0.22 & 0.10 & 0.04 & -1.34 & -0.62 & -0.27 \\
\hline
\end{tabular}

Conclusions about angular rate effects on the controllability of the subject aircraft are as follows:

- Non-zero nominal angular rates do not change control effectiveness, but do change mode shapes and/or speeds, as well as zero locations. 


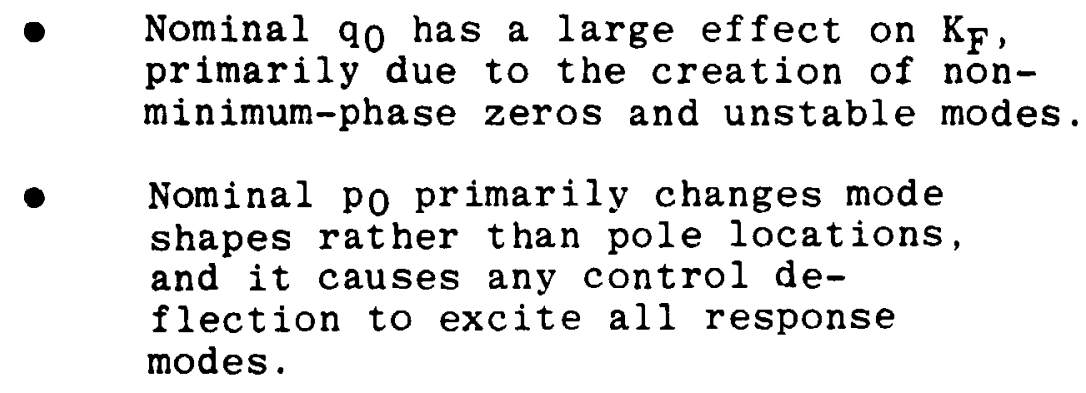

\title{
2.6 DYNAMIC VARIATIONS DURING EXTREME MANEUVERING
}

\begin{abstract}
Aircraft may be especially prone to departure from controlled flight during air combat maneuvering because such maneuvers are executed using the highest possible aircraft performance, and pilot workload during maneuvering flight is high. Although it is possible to fly most combat maneuvers in a smooth, coordinated manner, even small errors can cause difficulty due to instability, unfamiliar coupled mode shapes, or changes in control effectiveness.
\end{abstract}

Many air combat maneuvers include periods of high angle-of-attack flighi, in order to produce a large normal force for climbing or turning. High angular rates also are typical of many air combat maneuvers. High normal acceleration may be accompanied by large $\mathrm{q}_{0}$, and large $\mathrm{p}_{0}$ may be generated to rapidly orient the lift force in a desired direction.

Referring to the earlier sections in this chapter, the difficulties involved in extreme maneuvering become clear. High angles of attack and pitch rate destabilize the normal modes of motion and reduce the available control power, while high roll rate causes lateral-longitudinal coupling and produces mode shapes unfamiliar to the pilot. 
The first two of the following sections examine the changes in aircraft stability and control along two typical air combat trajectorif The third section appruaches the same problem from a different viewpoint, examining the effects of an elementary target-tracking pilot model on alrcraft stability.

\subsubsection{Wind-Up Turn}

In a wind-up turn, the alrcraft is rolled and high load factor is commanded, resulting in a high pitch rate. As airspeed bleeds cff (which may occur even at maximum thrust), angle of attack is increased, and the alrcraft stability decreases. Five points taken from a typical wind-up turn time history are described in Table 2.6-1, and the corresponding eigenvalues are given in Table 2.6-2. Of special interest is the Dutch roll damping, which decreases so that the Dutch roll mode becomes unstable as the wind-up turn progresses. These are not synuetric flight conditions, so it is expected that the Dutch roll eigenvector also contains angle-of-attack prrturbations.

In addition to lateral-longltudinal coupling and the general reduction in damping, the control effectiveness also decreases, as illustrated by the initial value of the transfer function, shown in Table 2.6-3. It is necessary for the pilot to use rudder as the roll control at high angles of attack, and this can cause sideslip perturbations, which cal lead to further problems.

These representative points from a wind-up turn demonstrate the deterioration of the stability and contrcl of the example aircraft as it executes one form of air combat maneuver. 
TABLE $\quad 2.6-1$

WIND-UP TURN WORKING POINTS

\begin{tabular}{|c|c|c|c|}
\hline $\begin{array}{c}\text { Working } \\
\text { Point }\end{array}$ & Description & \multicolumn{2}{|c|}{ Flight Condition } \\
\hline 1 & $\begin{array}{l}\text { Holl and Turn } \\
t=0 \mathrm{sec}\end{array}$ & $\begin{array}{l}v_{0}=217 \mathrm{ris} \\
p_{0}=5 \mathrm{deg} / \mathrm{sec} \\
\phi_{0}=45 \mathrm{deg}\end{array}$ & $\begin{array}{l}\alpha_{0}=5 \mathrm{deg} \\
r_{0}=5 \mathrm{deg} / \mathrm{sec} \\
{ }_{0}=5 \mathrm{deg}\end{array}$ \\
\hline 2 & $\begin{array}{l}\text { Rapid Turn } \\
t=13 \mathrm{sec}\end{array}$ & $\begin{array}{l}v_{0}=2: \mathrm{m} / \mathrm{s} \\
q_{0}=10 \mathrm{deg} / \mathrm{sec} \\
\phi_{0}=85 \mathrm{deg}\end{array}$ & $\begin{array}{l}\alpha_{0}=11 \mathrm{deg} \\
r_{0}=5 \mathrm{deg} / \mathrm{sec} \\
e_{0}=-15 \mathrm{deg}\end{array}$ \\
\hline 3 & $\begin{array}{l}\text { Turnang } \\
t=30 \mathrm{sec}\end{array}$ & $\begin{array}{l}v_{0}=2: 7 \mathrm{rz} / \mathrm{s} \\
a_{0}=20 \mathrm{deg} / \mathrm{sec} \\
\phi_{0}=70 \mathrm{deg}\end{array}$ & $\begin{array}{l}a_{0}=15 \mathrm{deg} \\
r_{0}=5 \mathrm{deg} / \mathrm{sec} \\
\theta_{0}=-20 \mathrm{deg}\end{array}$ \\
\hline 4 & $\begin{array}{l}\text { Turning } \\
t=52 \mathrm{sec}\end{array}$ & $\begin{array}{l}v_{0}=15 \mathrm{~m}: \mathrm{s} \\
p_{0}=10 \mathrm{deg} / \mathrm{sec} \\
r_{0}=10 \mathrm{deg} / \mathrm{sec} \\
\phi_{0}=70 \mathrm{deg}\end{array}$ & $\begin{array}{l}\alpha_{0}=22 \mathrm{deg} \\
q_{0}=15 \mathrm{deg} / \mathrm{sec} \\
\theta_{0}=-20 \mathrm{deg}\end{array}$ \\
\hline 5 & $\begin{array}{l}\text { Turning } \\
t=75 \mathrm{sec}\end{array}$ & $\begin{array}{l}v_{0}=126 \mathrm{r} \cdot \mathrm{s} \\
p_{0}=12.5 \mathrm{de} \cdot / \mathrm{sec} \\
\phi_{0}=60 \mathrm{deg}\end{array}$ & $\begin{array}{l}\alpha_{0}=27 \mathrm{deg} \\
q_{0}=12.5 \mathrm{deg} / \mathrm{sec} \\
{ }_{0}=-25 \mathrm{deg}\end{array}$ \\
\hline
\end{tabular}

TABLE. 2.6-2

WINL-UP TLRN EIGENYALULS

\begin{tabular}{|c|c|c|c|c|c|}
\hline $\begin{array}{c}\text { Working } \\
\text { Point }\end{array}$ & Short Perlod & Dutch Roll & Roll & Spiral & Phugold \\
\hline 1 & $-0.935: j 176$ & $-0.383: j 3.43$ & -161 & 0.064 & $-0.051 \pm j 0.126$ \\
2 & $-0.952: j 2.19$ & $-0.302 \pm j 4.37$ & -1.04 & -0.04 .3 & $0.002+j 0.191$ \\
3 & $-0.777: j 1.74$ & $-0.181+j 4.04$ & -0.826 & -0.074 & $-0012 \pm j 0.209$ \\
4 & $-0.456 \pm j 2.66$ & $0.0766: j 326$ & -0.758 & -0.096 & $-0.035 \pm j 0.327$ \\
5 & $-0.438 \pm j 2.16$ & $0.213 \pm \jmath 2.55$ & -0543 & -0.154 & $-0.057 \pm j 0.214$ \\
\hline
\end{tabular}


TABLE $2.6-3$

TRANSFER FUNCTION GAIN, $K_{I}$, ALONG THE WIND-UP TURN

\begin{tabular}{|c|c|c|c|c|c|c|c|}
\hline $\begin{array}{l}\text { Fork10g } \\
\text { Point }\end{array}$ & $\Delta w / \Delta \delta_{h}$ & $\Delta p / \Delta \delta_{a}$ & $\Delta p / \Delta \delta_{I}$ & $\Delta r / \Delta \delta_{a}$ & $\wedge r / \Delta \delta_{r}$ & $\Delta v / \Delta \delta_{a}$ & $\Delta v / \Delta \delta_{r}$ \\
\hline 1 & -0.42 & 15.80 & 3.80 & 0.321 & -2.87 & -0.013 & 0.127 \\
\hline 2 & -0.40 & 12.12 & 3.45 & 0.095 & -2.91 & -0.011 & 0.131 \\
\hline 3 & -0.49 & 5.91 & 2.14 & $-0.03 E$ & -2.03 & -0.002 & 0090 \\
\hline 4 & -0.31 & 1.32 & 1.08 & 0.037 & -1.06 & $0 \cdot 002$ & 0.048 \\
\hline 5 & -0.17 & -0.001 & 0.24 & 0.067 & -0.6 & 0.002 & c. .223 \\
\hline
\end{tabular}

\section{6 .2 Rolling Reversal}

A rolling reversal combines a rapid pull-up with a rapid rolling maneuver, resu,ting in a "corkscrew-like" path through space. The combination of a high-acceleration pullup and rapid rolling is expected to produce unstable modes with considerable lateral-longitudinal coupling. Table 2.6-4 describes the rollirig reversal working points examined here. The corresponding eigenvalues, shown in Table 2.6-5, illustrate the changes in aircraft stability as the rolling reversal progresses. Due to the $\mathrm{high}{ }^{a_{0}}$ involved in this muneuver, the Dutch roll mode is unstable throughout most of the maneuver.

The initial and final working points of the rolling reversal are symmetric flight conditions so there is no lateral-longitudinal colpling during these phases of the flight. This is demonstrated by the eigenvectors of the fast modes at the first working point, which are shown in Fig. 2.6-1. The intermediate working points all oceur cluring the aircraft's roll and involve significant lateral-longitudinal coupling. The eigenvecturs of the tast mirles at Wirking Pcint 3, shown in Fig. 2.6-1, demonstratw this Thers is significant 
TABLE 2.6-4

ROLLING REVERSAL WORKING POINTS

\begin{tabular}{|c|c|c|c|}
\hline $\begin{array}{l}\text { Working } \\
\text { Point }\end{array}$ & Description & \multicolumn{2}{|c|}{ Flight Condition } \\
\hline 1 & $\begin{array}{l}\text { Hi-G Pull-up } \\
t=0 \mathrm{sec}\end{array}$ & $\begin{aligned} v_{0} & =217 \mathrm{~m} / \mathrm{s} \\
q_{0} & =15 \mathrm{deg} / \mathrm{sec} \\
\theta_{0} & =30 \mathrm{deg}\end{aligned}$ & $\alpha_{0}=25 \mathrm{deg}$ \\
\hline 2 & $\begin{array}{l}\text { Roll } \\
t=4 \mathrm{sec}\end{array}$ & $\begin{array}{l}v_{0}=16,5 \mathrm{~m} / \mathrm{s} \\
p_{0}=-10 \mathrm{deg} / \mathrm{sec} \\
\phi_{0}=-90 \mathrm{deg}\end{array}$ & $\begin{array}{l}\alpha_{0}=26 \mathrm{deg} \\
q_{0}=15 \mathrm{deg} / \mathrm{sec} \\
\theta_{0}=50 \mathrm{deg}\end{array}$ \\
\hline 3 & $\begin{array}{l}\text { Roll } \\
t=10 \mathrm{sec}\end{array}$ & $\begin{array}{l}v_{0}=101 \mathrm{~m} / \mathrm{s} \\
p_{0}=-15 \mathrm{deg} / \mathrm{sec} \\
r_{0}=-5 \mathrm{deg} / \mathrm{sec} \\
{ }_{0}=-30 \mathrm{deg}\end{array}$ & $\begin{array}{l}\alpha_{0}=26 \mathrm{deg} \\
q_{0}=15 \mathrm{deg} / \mathrm{sec} \\
\phi_{0}=-180 \mathrm{deg}\end{array}$ \\
\hline 4 & $\begin{array}{l}\text { Roll and Pull-up } \\
t=15 \mathrm{sec}\end{array}$ & $\begin{array}{l}v_{0}=117 \mathrm{~m} / \mathrm{s} \\
p_{0}=10 \mathrm{deg} / \mathrm{sec} \\
r_{0}=5 \mathrm{deg} / \mathrm{sec} \\
\theta_{0}=-55 \mathrm{deg}\end{array}$ & $\begin{array}{l}\alpha_{0}=23 \mathrm{deg} \\
q_{0}=15 \mathrm{deg} / \mathrm{sec} \\
\phi_{0}=45 \mathrm{deg}\end{array}$ \\
\hline 5 & $\begin{array}{l}\text { Final Pull-up } \\
t=22 \mathrm{sec}\end{array}$ & $\begin{array}{l}v_{0}=152 \mathrm{~m} / \mathrm{s} \\
p_{0}=0 \mathrm{deg} / \mathrm{sec} \\
\phi_{0}=0 \mathrm{deg}\end{array}$ & $\begin{array}{l}\alpha_{0}=20 \mathrm{deg} \\
a_{0}=10 \mathrm{deg} / \mathrm{sec} \\
\theta_{0}=-10 \mathrm{deg}\end{array}$ \\
\hline
\end{tabular}

TABLE 2.6-5

ROLLING REVERSAL EICENVALUES

\begin{tabular}{|c|c|c|c|c|c|}
\hline $\begin{array}{l}\text { Working } \\
\text { Point }\end{array}$ & Short Period & Dutch Roll & Ro11 & Spiral & Phugo id \\
\hline 1 & $-0.873 \pm j 4.07$ & $0.191 \pm j 4.54$ & -0.910 & 0.139 & $-0.178 \quad-0.056$ \\
\hline 2 & $-0.691 \pm j 3.15$ & $0.180 \pm J 3.55$ & -0.716 & $-c .169$ & $0.0124 \pm j 0.406$ \\
\hline 3 & $-0.403 \pm j 1.91$ & $0.107 \pm \mathrm{J} 2.10$ & -0.409 & -0.224 & $0.122 \pm j 0.124$ \\
\hline 4 & $-0.394 \pm j 2.34$ & $0.131 \pm j 2.70$ & -0.711 & -0.093 & $-0.253 \pm j 0.124$ \\
\hline 5 & $-u .593 \pm j 2.78$ & $-0.010 \pm \mathrm{J} 3.83$ & -0.818 & -0.082 & $-0.073 \pm j 0.095$ \\
\hline
\end{tabular}




\section{DUTCH ROLL}

1

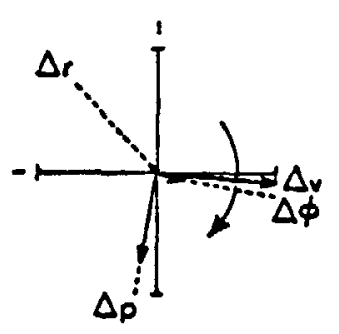

3

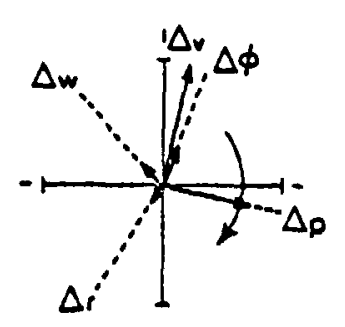

SHORT PERIOO
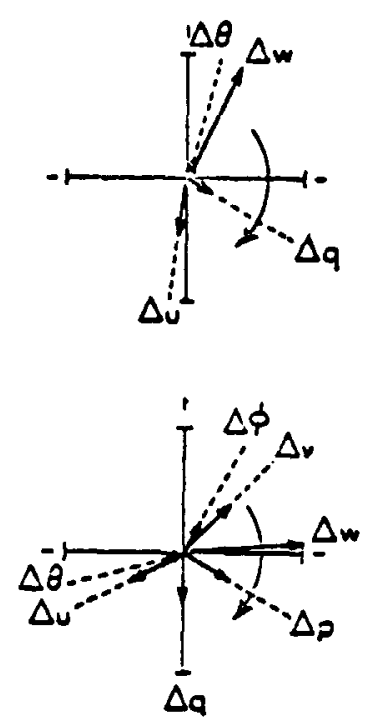

ROL
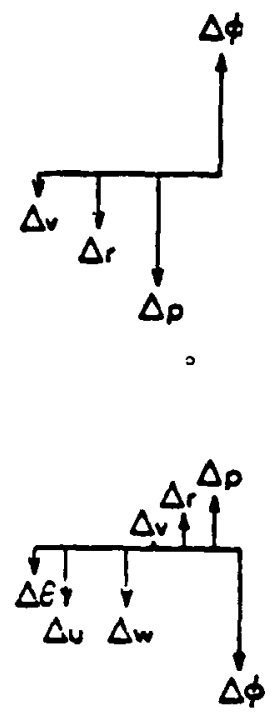

Figure 2.6-1 Eigenvectors of Rolling Reversal

angle-of-attack (or $\Delta w$ ) motion in the Dutch roll mode and large roll-sideslip perturbation in the short period mode. Even the roll mode contains a significant angle-of-attack excursion.

The aircraft control effectiveness follows trends similar to the aircraft stability, i.e., the control effectiveness is degraded throughout the first half of the maneuver, but it improves during the second half, as illustrated in Table 2.6-6. The pilot must use the sudder as a roll control during the middle portion of this maneuver. This difficulty is complicated by high angular rates, lateral-longitudinal coupling, and extreme attitudes.

An aircraft executing a rolling reversal exhibits unstable modes, lateral-longitudinal coupling, and reduced control effectiveness as the maneuver progresses. all of which make the pilot's task more difficult. 
TABLE 2.6-6

TRANSFER FUNCTION GAIN, $\mathrm{K}_{I}$, ALONG THE ROLLING REVERSAL

\begin{tabular}{|c|c|c|c|c|c|c|c|}
\hline $\begin{array}{l}\text { Working } \\
\text { Point }\end{array}$ & $\Delta w / \Delta \delta_{h}$ & $\Delta p / \Delta \delta_{\mathrm{a}}$ & $\Delta p / \Delta \delta_{r}$ & $\Delta \mathrm{r} / \Delta \delta_{\mathrm{a}}$ & $\Delta r / \Delta \delta_{r}$ & $\Delta v / \Delta \delta_{g}$ & $\Delta v / \Delta \sigma_{T}$ \\
\hline 1 & -0.62 & 1.87 & 1.53 & 0.204 & -2.25 & 0.004 & 0.109 \\
\hline 2 & -0.36 & 0.597 & 0.781 & 0.142 & -1.29 & 0.003 & 0.063 \\
\hline 3 & -0.012 & -0.001 & 0.282 & 0.053 & -0.465 & 0.001 & 0.023 \\
\hline 4 & -0.23 & 0.683 & 0.722 & 0.044 & -0.771 & 0.001 & 0.036 \\
\hline 5 & -0.42 & 2.81 & 1.73 & -0.031 & -1.313 & 0.002 & 0.056 \\
\hline
\end{tabular}

\subsubsection{Effects of Proportional Tracking}

Rudimentary piloting effects can be examined by assuming that the pilot attempts to control the aircraft's attitude. This can be modeled by a proportional feedback of angular deviation (pitch or roll angle) to the appropriate control surface (elevator or aileron). Pilot lags or time delays are neglected. As an example, pitch attitude control is chosen, and ihe feedback gains are set so that the effective pitch moment due to pitch angle, $M_{\theta}$, is a multiple of the pitch moment due to angle of attack, $M_{\alpha}$. Since this is achieved by elevator feedback, there are changes to the coefficients $x_{\theta}$ and $z_{\theta}$ as wel]. The multiplying factor is denoted by " $i$ " in the following tables, and the feedback gain that produces equal $M_{\theta}$ and $M_{\alpha}(i=1)$ for the reference flight condition is $0.64 \mathrm{deg}$ elevator per deg of pitch angle. This simple model also disregards pitch-rate feedback, which the pilot also might normally provide.

For symmetric flight conditions, this loop closure does not affect the lateral eigenvalues; the longitudinal eigenvalue variations are shown in Table 2.6-7. The shortperiod mode is both increased in frequency and decreased in 
TABLE 2.6-7

EIGENVALUE CHANGES DUE TO PROPORTIONAL TRACKING -SYMMETRIC FLIGHT CONDITIONS

\begin{tabular}{|c|c|c|c|}
\hline \multicolumn{2}{|c|}{$v_{0}=94 \mathrm{~m} / \mathrm{s}$} & $\alpha_{0}=1$ & $\mathrm{q}_{0}=0 \mathrm{de}$ \\
\hline 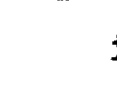 & Short & Period & $\begin{array}{c}\text { Phugoid (Pitch } \\
\text { Argle/Speed) }\end{array}$ \\
\hline ( & -0.407 & $\pm j 1.099$ & $-0.017 \pm j 0.137$ \\
\hline 1 & -0.342 & $\pm j 1.552$ & $-0.082 \pm j 0.068$ \\
\hline 2 & -0.319 & $9 \pm j 1.906$ & -0.0522 \\
\hline$\leq$ & -0.302 & $2 \pm j 2.47$ & -0.0260 \\
\hline
\end{tabular}

\begin{tabular}{|c|cc|c|}
\hline \multicolumn{2}{|c|}{$v_{0}=94 \mathrm{~m} / \mathrm{s} \quad \alpha_{0}=15 \mathrm{deg} \quad \mathrm{q}_{0}=12 \mathrm{deg} / \mathrm{sec}$} \\
\hline$i$ & Short Period & \multicolumn{2}{|c|}{$\begin{array}{c}\text { Phugoid (Pitch } \\
\text { Angle/Speed) }\end{array}$} \\
\hline 0 & $-0.417 \pm j 1.13$ & 0.045 & -0.069 \\
1 & $-0.351 \pm j 1.57$ & -0.096 & -0.058 \\
2 & $-0.327 \pm j 1.92$ & -0.140 & -0.063 \\
4 & $-0.307 \pm j 2.48$ & -0.178 & -0.064 \\
\hline
\end{tabular}

damping by the addition of a pitch attitude-to-elevatcr

feedback. For straight-and-level flight, attitud control increases the phugoid damping while decreasing th stural frequency, resulting in the conversion of the phugoid mode into two real modes -- a pitching mode and a speed mode. In steady pitching motion, pitch-attitude control results in increased stability for the pitch angle mode with relatively little effect on the speed mocie.

Coupled flight conditions lead to significant effects on the lateral modes due to the longitudinal loop closure. In Ref. 9, a pitch attitude-to-elevator loop 
closure resulted in an unstable lateral mode when the subject aircraft was in a steady sideslip. As shown in Table 2.6-8 for the example airsraft used in this report, pitch angle-to-elevator feedback generally has a beneficial influence on the lateral eigenvalues when this aircraft is rolling at zero sideslip. However, the presence of a nonzero nominal sideslip angle results in a mildly diverging speed mode for moderate-to-large feedback gains. There is an inaication that large attitude feedback gains destabilize the Dutch roll mode (for a non-rolling aircraft) or the short period mode (when the aircraft is rolling and slipping).

Regarding the pitch attitude-to-elevator feedback as a simple pilot model, this examination confirms the earlier result that pilot control of the longitudinal motion of an aircraft could result in the destabilization of the aircraft when the vehicle is in a steady sideslip. This would be due to the pilot disregarding the lateral-longitudinal coupling present in asymmetric flight conditions.

This simple attitude feedback underlines the necessity for considering lateral-longitudinal crossfeeds when designing a stabllity augmentation system for a highperformance aicraft. To achieve acceptable performance, it may be necessary to design a system that recognizes the aircraft flight condition and adjusts its gains to suit the situation.

\subsection{CHAPTER SUMMARY}

This chapter has presented a study of the dynamic charac əristics of a high-performance aircraft, with special emphasis on the effects of extreme flight conditions on 
TABLE $2.6-8$

EIGENVALUE CHANGES DUE TO PROPORTIONAL TRACKING -. SIDESLIP AND ROLL EFFECTS

\begin{tabular}{|c|c|c|c|c|c|c|}
\hline \multicolumn{2}{|r|}{$\mathrm{v}_{0}=94 \mathrm{~m} / \mathrm{s}$} & $\alpha_{0}=15 \mathrm{deg}$ & \multicolumn{2}{|c|}{$B_{0}=0 \mathrm{deg}$} & \multicolumn{2}{|c|}{$=0 \mathrm{deg} / \mathrm{sec}$} \\
\hline $\mathbf{i}$ & Short Period & Dutch Roll & Rol1 & Spiral & Phu & id \\
\hline 0 & $-0.407 \pm j 1.099$ & $0.0738 \pm j 2.25$ & -0.442 & -0.0545 & -0.017 & j0.137 \\
\hline 1 & $-0.342 \pm j 1.552$ & $0.0738 \pm j 2.25$ & -0.442 & -0.0545 & -0.082 & j0.068 \\
\hline 2 & $-0.319 \pm j 1.906$ & $0.0738 \pm j 2.25$ & -0.442 & -0.0545 & -0.157 & -0.0522 \\
\hline 4 & $-0.302 \pm j 2.47$ & $0.0738 \pm j 2.25$ & -0.442 & -0.0545 & -0.218 & -0.0260 \\
\hline
\end{tabular}

\begin{tabular}{|l|l|l|l|l|l|}
\hline \multicolumn{2}{|c|}{$v_{0}=94 \mathrm{~m} / \mathrm{s}$} & $\alpha_{0}=15 \mathrm{deg}$ & $\beta_{0}=10 \mathrm{deg} \mathrm{p}_{w_{0}}=0 \mathrm{deg} / \mathrm{sec}$ \\
\hline $\mathrm{i}$ & Short Period & Dutch Roll & Roll & Spiral & Phugoid \\
\hline \hline 0 & $-0.353 \pm j 1.368$ & $-0.134 \pm j 2.11$ & -0.434 & -0.0315 & $-0.024 \pm j 0.146$ \\
1 & $-0.303 \pm j 1.76$ & $-0.153 \pm j 2.11$ & -0.431 & -0.081 & $-0.032 \pm j 0.124$ \\
2 & $-0.298 \pm j 2.15$ & $-0.143 \pm j 2.03$ & -0.430 & -0.021 & -0.287 \\
4 & $-0.327 \pm j 2.63$ & $-0.101 \pm j 2.08$ & -0.428 & -0.152 & $-0.0247 \pm j 0.108$ \\
\hline
\end{tabular}

\begin{tabular}{|c|c|c|c|c|c|}
\hline \multicolumn{2}{|c}{$v_{0}=94 \mathrm{~m} / \mathrm{s}$} & $\alpha_{0}=15 \mathrm{deg}$ & $\beta_{0}=10 \mathrm{deg} \quad \mathrm{p}_{w_{0}}=-39 \mathrm{deg} / \mathrm{sec}$ \\
\hline $\mathrm{i}$ & Short Period & Dutch Roll & Roll Spiral & Phugoid \\
\hline \hline 0 & $-0.511 \pm j 1.32$ & $-0.057 \pm j 2.28$ & $-0.246 \pm j 0.199$ & $0.071 \pm j 0.207$ \\
1 & $-0.349 \pm j 1.58$ & $-0.116 \pm j 2.34$ & $-0.157 \pm j 0.413$ & -0.193 & -0.050 \\
2 & $-0.201 \pm j 1.77$ & $-0.202 \pm j 2.44$ & $-0.215 \pm j 0.480$ & -0.249 & -0.0035 \\
4 & $-0.038 \pm j 1.93$ & $-0.306 \pm j 2.80$ & $-0.270 \pm j 0.518$ & -0.275 & 0.0155 \\
\hline
\end{tabular}

\begin{tabular}{|c|c|c|c|c|c|}
\hline \multicolumn{2}{|c|}{$v_{0}=94 \mathrm{~m} / \mathrm{s}$} & $\alpha_{0}=15 \mathrm{deg}$ & $B_{0}=0 \mathrm{deg} \quad p_{w_{0}}=-39 \mathrm{deg} / \mathrm{sec}$ \\
\hline $\mathrm{i}$ & Short Period & Dutch Rol 1 & Roll spiral & Phugoid \\
\hline 0 & $-0.464 \pm j 1.29$ & $-0.032 \pm j 2.34$ & $-0.293 \pm j 0.144$ & $0.043 \pm j 0.189$ \\
1 & $-0.400 \pm j 1.56$ & $-0.044 \pm j 2.37$ & $-0.167 \pm j 0.500$ & -0.207 & -0.062 \\
2 & $-0.334 \pm j 1.82$ & $-0.073+j 2.42$ & $-0.208+j 0.600$ & -0.229 & -0.035 \\
4 & $-0.169 \pm j 2.10$ & $-0.201 \pm .12 .67$ & $-0.246 \pm j 0.666$ & -0.238 & -0.0228 \\
\hline
\end{tabular}


aircraft stability and control. The chapter first examines previous studies of the dynamics, aerodynamics, and control in this flight environment. The difficulty of measuring angular rate and translational acceleration effects leads to limited availability of this aerodynamic data, which has a significant impact on the simulation and analysis of dynamic departures. The survey of stability and control indicates a need for additional developments in these areas. 
3. EFFECTS OF CONFIGURATION VARIATIONS ON AIRCRAFT DYNAMICS

\subsection{OVERVIEW}

Variations of aircraft configuration lead to changes in the aircraft's eigenvalues, eigenvectors, and control effectiveness. Section 3.2 presents the effects of changes in the most important longitudinal stability derivatives on the mode shapes and speeds. Similar effects caused by changes in lateral stability derivatives are detailed in Section 3.3. The effects of aircraft mass and rotational inertia variations are given in Section 3.4. Section 3.5 presents a general discussion of possible departure modes and illustrates some of the possible departure time histories. Section 3.6 is a summary of the chapter.

\subsection{VARIATIONS DUE TO LONGITUDINAL STABILITY DERIVATIVES}

The longitudinal stability derivatives determine the aerodynamic force and moment contributions to the longitudinal perturbation equations, and these stability derivatives can vary considerably from aircraft to aircraft. This section surveys the changes in normal mode shapes and speeds for different ranges of the most important longitudinal stability derivatives.

Three aerodynamic derivatives dominate the short period motion of the aircraft: $C_{m_{q}}, C_{m_{\alpha}}$, and $C_{Z_{\alpha}}$. The significance of these terms can be seen in reduced-order approximations to the damping ratio, $;$, and natural frequency, $\omega_{n}$, of this mode: 


$$
\begin{aligned}
& \zeta \cong-\frac{1}{\delta I_{y} \omega_{n}} \rho_{0} v_{0} s^{-2}\left[c_{m_{q}}+\frac{2 I y}{c^{2} m} c_{z_{\alpha}}\right] \\
& \omega_{n} \cong\left[-\frac{1}{2 I_{y}} \rho_{0} v_{0}^{2} s \bar{c}\left(c_{m_{\alpha}}-\frac{1}{4 m} \rho_{0} s \bar{c} c_{m_{q}} c_{z_{\alpha}}\right)\right]^{\frac{1}{2}}
\end{aligned}
$$

Over the range of likely values of the coefficients, the $c_{m_{q}} c_{Z_{a}}$ product usually is considerably smaller than $C_{m_{\alpha}}$, so it can be expected that $C_{m_{q}}$ and $C_{z_{\alpha}}$ primarily affect short period damping, while $C_{m_{\alpha}}$ changes only the short period natural frequency.

The specific effects of varying $C_{Z_{\alpha}}$ and $C_{m_{q}}$ are illustrated in Table 3.2-1. The primary short period eigenvalue changes occur in the damping, as expected. The phugoid mode does change somewhat, as an increase in the lift-curve slope $\left(\mathrm{C}_{\mathrm{Z}_{\alpha}}\right.$ more negative) increases the phugoid natural frequency at essentially constant damping ratio. An increase in the magnitude of pitch damping $\left(\mathrm{C}_{\mathrm{m}_{\mathrm{q}}}\right)$ decreases phugoid frequency and damping significantly.

TABLE 3.2-1

$\mathrm{C}_{\alpha}$ AND $\mathrm{C}_{\mathrm{m}_{\mathrm{q}}}$ EFFECTS ON E:GENVALUES $\left(v_{0}=94 \mathrm{~m} / \mathrm{s}, \quad \alpha_{0}=15 \mathrm{deg}\right)$

\begin{tabular}{|c|c|c|}
\hline$C_{Z_{\alpha}}\left(\mathrm{rad}^{-1}\right)$ & Short Period & Phugoid \\
\hline \hline $\begin{array}{c}-2.0 \\
-3.2\end{array}$ & $-0.345 \pm j 1.096$ & $-0.0146 \pm j 0.128$ \\
-5.0 & $-0.396 \pm j 1.099$ & $-0.0162 \pm j 0.135$ \\
\hline $\mathrm{C}_{\mathrm{m}_{\mathrm{q}}\left(\mathrm{rad}^{-1}\right)}$ & Short Period & Phugoid \\
\hline \hline-34.4 & $-0.642 \pm j 1.058$ & $-0.013 \pm j 0.130$ \\
-17.2 & $-0.399 \pm j 1.099$ & $-0.017 \pm j 0.137$ \\
-5.73 & $-0.236 \pm j 1.098$ & $-0.020 \pm j 0.143$ \\
\hline
\end{tabular}


Variations in the longitudinal stability derivatives affect the lateral modes only when the aircraft is in asymmetric flight. In that case, mode coupling occurs-Table 3.2-2 illustrates the changes in the Dutch roll and short period modes for asymmetric flight as $C_{m_{q}}$ varies. The transfer of damping from the short period mode to the Dutch roll mode for non-zero $B_{0}$ has been observed in Section 2.4 . In the case of reduced $C_{m_{q}}$, this transfer is essentially unchanged, indicating that short period damping due to $\mathrm{CZ}_{\alpha}$ (see Eq. $(3.2-1)$ ) is transferred whereas damping due to $\mathrm{C}_{\mathrm{m}_{\mathrm{q}}}$ is not. This is supported by the observation that Dutch roll eigenvectors for non-zero $\beta_{0}$ (Fig. 2.4-4) include much more angle-of-attack motion than pitch rate.

TABLE $3.2-2$

EFFECTS OF $\mathrm{C}_{\mathrm{m}_{\mathrm{q}}}$ ON EIGENVALUES IN ASYMMETRIC FLIGHT

\begin{tabular}{|c|c|c|}
\hline \multicolumn{3}{|c|}{$\alpha_{0}=15 \mathrm{deg} \quad{ }_{0}{ }_{0}=0 \mathrm{deg} \quad p_{w_{0}}=0 \mathrm{deg} / \mathrm{sec}$} \\
\hline & Short Period & Dutch Roll \\
\hline $\begin{array}{l}c_{m_{q}}=-17.8\left(\mathrm{rad}^{-1}\right) \\
C_{m_{q}}=-5.73\left(\mathrm{rad}^{-1}\right)\end{array}$ & $\begin{array}{l}-0.407 \pm j 1.099 \\
-0.236 \pm j 1.098\end{array}$ & $\begin{array}{l}-0.074 \pm j 2.251 \\
-0.074 \pm j 2.251\end{array}$ \\
\hline \multicolumn{3}{|c|}{$\alpha_{0}=15 \mathrm{deg} \quad \beta_{0}=5 \mathrm{deg} p_{w_{0}}=0 \mathrm{deg} / \mathrm{sec}$} \\
\hline & Short Period & Dutch Roll \\
\hline$C_{m_{\mathrm{q}}}=-17.8\left(\mathrm{rad}^{-1}\right)$ & $-0.382 \pm j 1.201$ & $-0.0946 \pm . j 2.024$ \\
\hline$c_{m_{q}}=-5.73\left(\mathrm{rad}^{-1}\right)$ & $-0.205 \pm j 1.205$ & $-0.0956 \pm j 2.024$ \\
\hline
\end{tabular}

\begin{tabular}{|c|c|c|}
\hline$a_{0}=15 \mathrm{deg}$ & $\mathrm{p}_{\mathrm{w}_{0}}=-3$ & $96 \mathrm{deg} / \mathrm{sec}$ \\
\hline & Short Period & Dutch Rc1l \\
\hline$C_{m_{q}}=-17.8\left(\mathrm{rad}^{-1}\right)$ & $-0.464 \pm j 1.294$ & $-0.0319 \pm j 2.342$ \\
\hline$C_{m_{q}}=-5.73\left(\mathrm{rad}^{-1}\right)$ & $-0.291 \pm j 1.295$ & $-0.0343 \pm j 2.337$ \\
\hline
\end{tabular}


None of the stability derivatives discussed has any effect on the control effectiveness, which determines the transfer function gain, $K_{I}$; therefore, the initial response to control inputs does not vary with $\mathrm{Cz}_{\alpha}$ and $\mathrm{C}_{\mathrm{m}_{\mathrm{q}}}$. Hcwever, stability derivative variations do affect the transient response through changes in poles and zeros.

Variations in the aircraft's center of gravity cause variations in aerodynamic moment coefficients. The center of gravity (c.g.) is the rotational center of the alrcraft. For fixed aerodynamic center of pressure, c.g. variation leads to static margin variation; hence, the moment relationships are altered.

The static margin is the distance between the c.g. location and the aerodynamic center, and it is usually expressed as a fraction of the mean serodynamic chord, $\bar{c}$. Figure 3.2-1 details the changes in the longitudinal eigenvalues as the static margin is varied from 0.33 through its usual reference location of 0.17 to -0.15 . This results in a $C_{m_{\alpha}}$ variation from -1.17 to $0.554 \mathrm{rad}^{-1}$, as well as changes in $\mathrm{C}_{\mathrm{m}_{\mathrm{q}}}$ from ${ }^{-0}$ $-21.1 \mathrm{rad}^{-1}$ to $-11.2 \mathrm{rad}^{-1}$.

The eigenvectors (Fig. 3.2-2) change considerably as the c.g. moves aft. While the short period and phugoid modes still are recognizable at a static margin of 0.06 $\left(C_{m_{\alpha}}=-0.17 \mathrm{rad}^{-1}\right)$, a transition region is entered as the c.g. moves further aft. At a staic margin of 0.01 $\delta C_{m_{\alpha}}=-0.02 \mathrm{rad}^{-1}$ ), a new ("third") oscillatory mode which dis:lays significant perturbations in all longitudinal states is evident. Two real convergences comprise the other longitudinal modes - - one fast attitude mode occurring at constant veiocity and flight path angle and one slow velocity mode that involves significant flight path angle variations. 


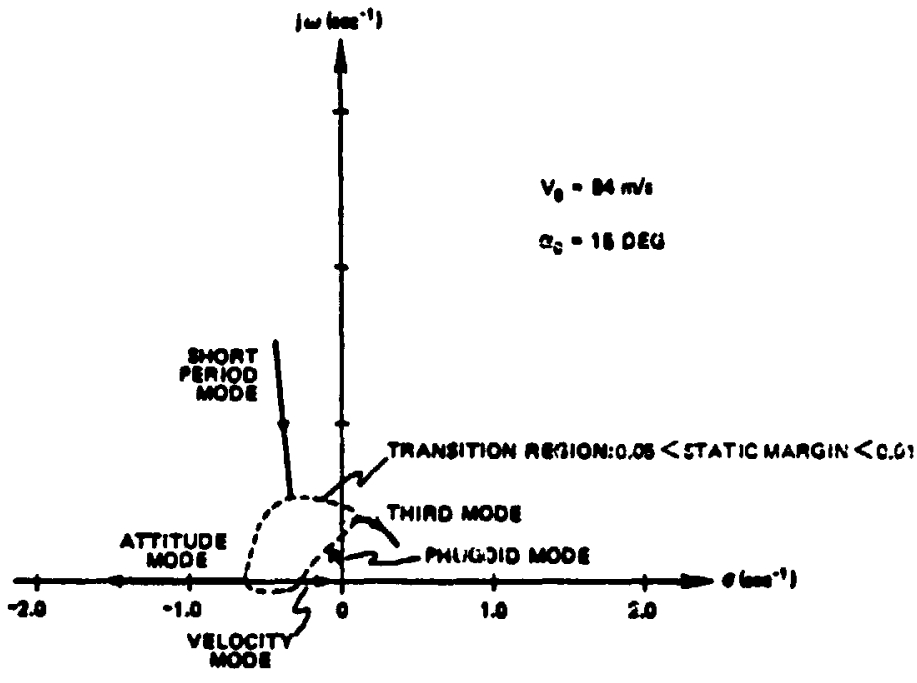

Figure 3.2-1 Longitudinal Eigenvalue Variations with c.g. Location

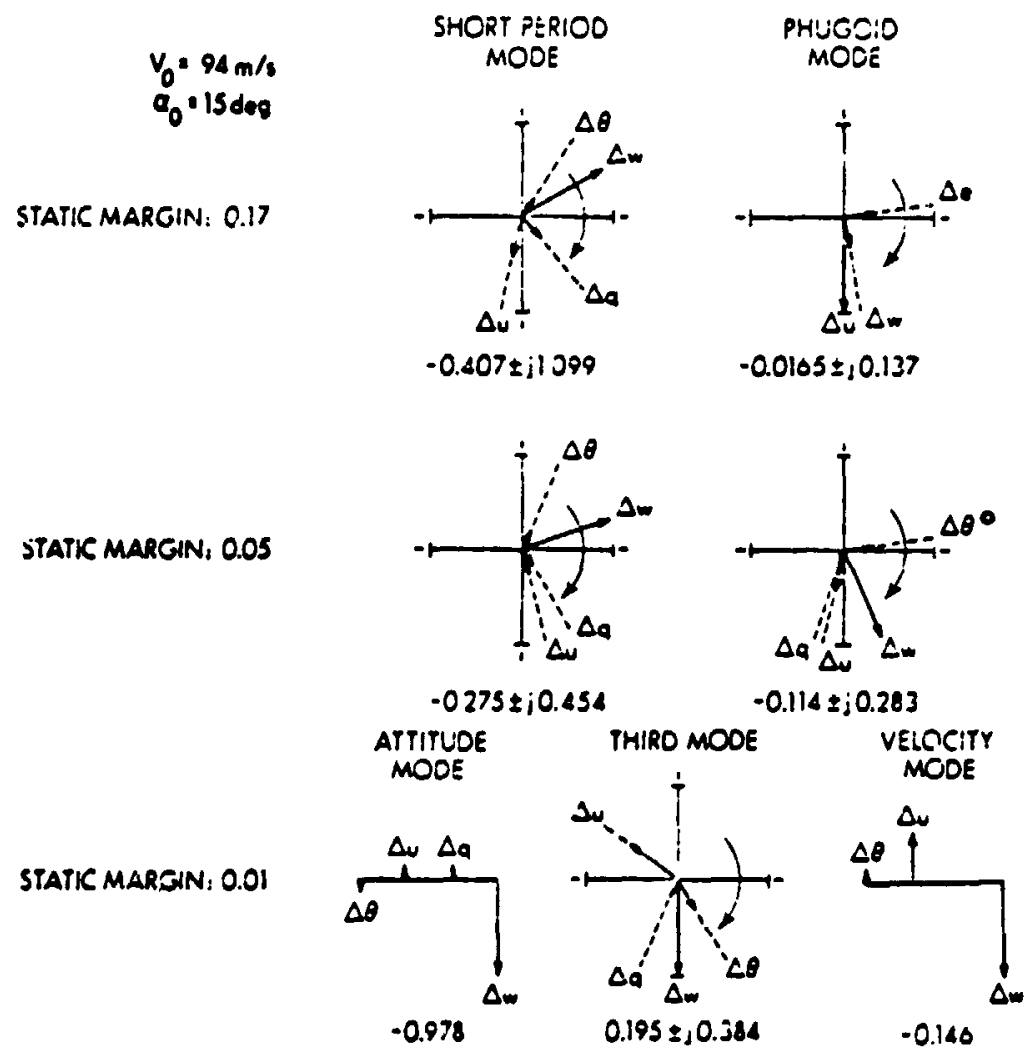

Figure 3.2-2 Longitudinal Eigenvector Variations with c.g. Location 
Control effectiveness changes only slightly with c.g. location, but the iransfer function gain, $K_{F}$, does vary as shown in Table 3.2-3. The zeros of the $\Delta w / \Delta \delta_{h}$ transfer function are given in Table 3.2-4. Both of these tables indicate the well-behaved nature of the numerator of the elevator-to-angle of attack transfer function.

TABLE 3.2-3

C.G. LOCATION EFFECTS ON TRANSFER FUNC' ${ }_{1}$ ION GAIN, $\mathrm{K}_{F}$

$$
\left(\mathrm{V}_{0}=94 \mathrm{~m} / \mathrm{s}, \alpha_{0}=15 \mathrm{deg}\right)
$$

\begin{tabular}{|c|c|c|c|}
\hline Static Margin & $\Delta w / \Delta \delta_{h}$ & $\Delta p / \Delta \delta_{a}$ & $\Delta r / \Delta \delta_{r}$ \\
\hline 0.17 & -5.17 & -0.66 & -5.39 \\
0.05 & -5.76 & -0.57 & -5.51 \\
-0.03 & -6.15 & -0.51 & -5.59 \\
\hline
\end{tabular}

TABLE $3.2-4$

COMPARISON OF ZEROS OF $\triangle W / L \delta_{h} A T$

THRFE, C.G. LOCATIONS

\begin{tabular}{|c|c|c|}
\hline Static Margin & $z_{1}, 2$ & $z_{3}$ \\
\hline 0.17 & $-0.0025 \pm j 0.1167$ & -21.07 \\
0.05 & $-0.0038 \pm j 0.1238$ & -20.998 \\
-0.03 & $-0.0047 \pm j 0.1283$ & -20.950 \\
\hline
\end{tabular}

This section examines the effects of longitudinal stability derivatives, and the following conclusions are made : 
- Reductions in pitch damping $\left(\mathrm{C}_{\mathrm{m}_{\mathrm{q}}}\right)$ and lift-curve slope $\left(C_{Z_{\alpha}}\right)$ reduce the short period damping without changing the frequency.

- Lateral-longitudinal coupling produced by sicleslip transfers damping due to $C_{z_{\alpha}}$ to the Dutch roll mode from the shoit period mode, but damping due to $\mathrm{C}_{\mathrm{m}_{\mathrm{q}}}$ remains in the longitudinal plane.

- The c.g. location affects $\mathrm{C}_{\mathrm{m}_{\alpha}}$ directly, and a rearward c.g. location results in the creation of a new unstable uscillatory mode that exhibits significant perturbations in all longitudinal variables. In addition, two stable real modas are created -- a fast attitude mode and a slow velocity mode.

- The numerator of the elevator-to-anrle of attack transfer function is well behaved at rearward c g. locations.

\subsection{VARIATIONS DUE TO LATERAL-DIRECTIONAL STAEILITY DERIVATIVES}

In this section, variations in $C_{l_{B}}, C_{n_{r}}, C_{l_{p}}$, and $C_{n_{r}}$ are studied to determine their effects on the lateraldirectionai modes. Experimental inforilation on the value of $\mathrm{C}_{\mathbf{n}_{\dot{\beta}}}$ is limited, so an investigation of possibie effects of $\mathrm{C}_{\mathrm{n}_{\xi}}$ on eigenvalues is included in this section.

The enfects of $C_{n_{E}}, C_{I_{B}}$, and $C_{l_{p}}$ variations are shown in Fig. 3.3-1. Increas 0 . in magnitude $c: C_{n_{B}}$ and $C_{l_{B}}$ cause an increase in the frequency of the Dutch roll mode. Additionally, both parameters cause some change in damping, with larger $C_{n_{B}}$ increasing the damping ratio and larger $\mathrm{C}_{1_{B}}$ magnitude decreasing the Dutch roll damping $\boldsymbol{s}$ atio. Larger $C_{1_{B}}$ magnitude causes the piral mude to re more stable. 


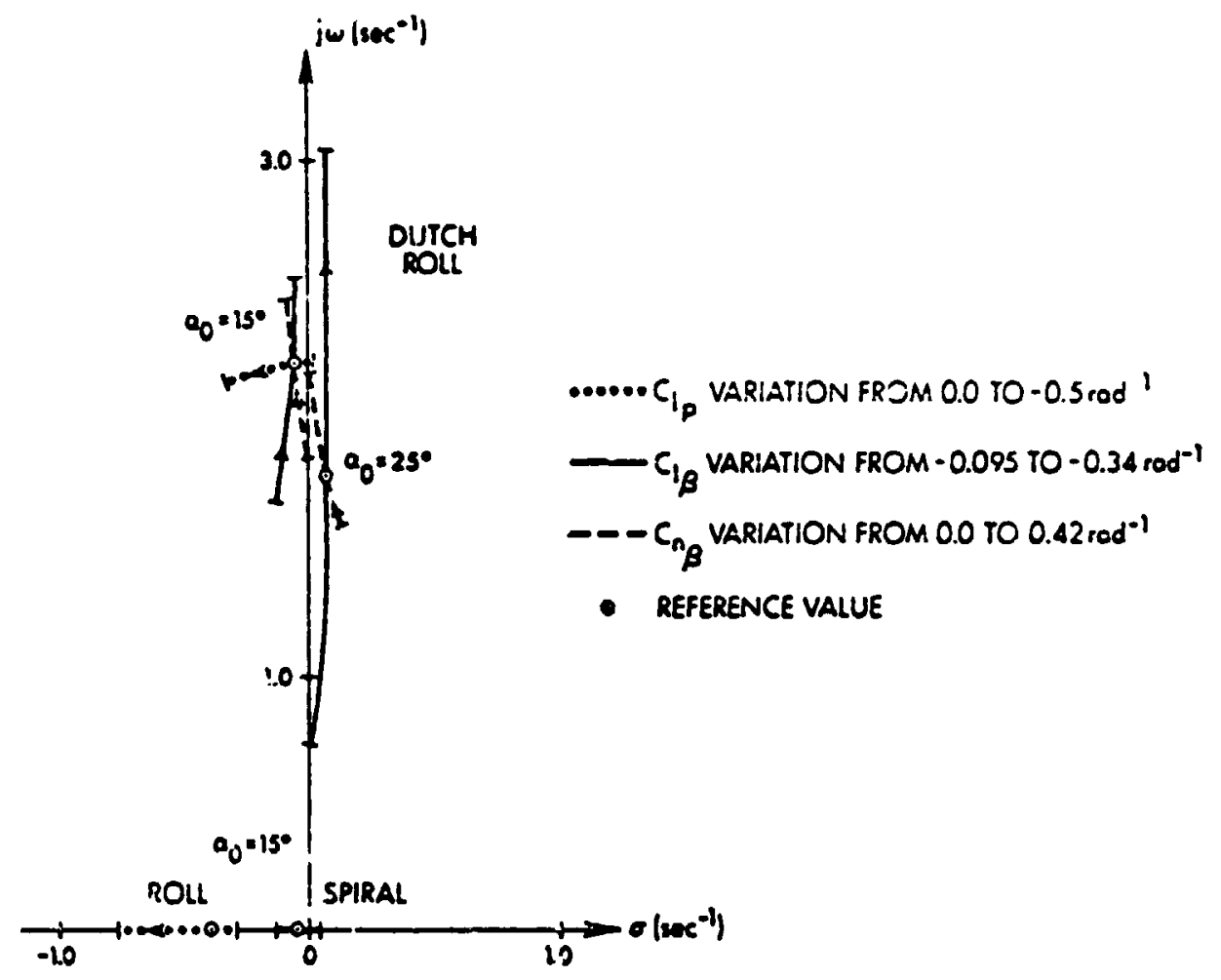

Figure 3.3-1 Effects of $c_{1_{\beta}}, C_{n_{\beta}}$, and $c_{I_{p}}$ Variations on Lateral-Directional Eigenvalues

Figure 3.3-1 indicates that at $\alpha_{0}$ of 15 deg the effects of $C_{l_{p}}$ appear in the Dutch roll damping ratio and in the roll corvergence mode. The presence of these effects is predicted by the apprcximate lateral-directional equations, Eqs. (2.4-1) to (2.4-5), but the approximate equations give an inaccurate indication of the size of these effects. Equations (2.4-1) and (2.4-4) predict much larger variation in the roll mode eigenvelue due to $c_{1}$ variation than is indicated in Fig. 3.4-1. Conversely, Eqs. (2.4-2) and (2.4-5) predict a much smaller variation in Dutch roll damping ratic than occurs in the complete model of the subject aircraft. 
The lack of accuracy of the approximate equations results from the fact that the subject aircraft does not conform to the assumptions upon which the approximate equations are based. The subject aircraft exhibits much more roll motion in Dutch roll mode (as shown by the eigenvectors in Fig. 2.4-3) because it rolls easily (due to low $I_{x} / I_{z}$ ratio) and because its roll-yaw and rollsideslip cross derivatives $\left(C_{1_{r}}, C_{n_{p}}\right.$, and $\left.C_{l_{\beta}}\right)$ are not small when properly compared to the roll and yaw-sidesiip derivatives $\left(C_{1_{p}}, C_{n_{r}}\right.$, and $\left.C_{n_{\beta}}\right)$.

Table 3.3-1 compares the lateral-directional eigenvalues for $C_{n_{B}}$ and $C_{1_{B}}$ variations in the presence of nonzero nominal pitch rate. A comparison of the eigenvalues indicates that the effects of steady pitching are independent of the changes in aerodynamic coefficients. In all cases, positive pitch rate destabilizes the Dutch roll and sfiral modes and speeds up the roll mode.

TABLE $3.3-1$

EFFECTS OF $C_{n_{\beta}}$ AND $C_{1_{\beta}}$ VARIATIONS IN THE PRESENCE OF STEADY PITCH RATE

\begin{tabular}{|c|c|c|c|c|}
\hline & & Dutch Rol1 & $\mathrm{Rcll}$ & Spiral \\
\hline $\begin{array}{l}\text { Referen: } \\
\text { Values }\end{array}$ & $\begin{array}{l}\mathrm{q}_{0}=0 \\
\mathrm{q}_{0}=12 \mathrm{deg} / \mathrm{sec}\end{array}$ & $\begin{array}{c}-0.0738 \pm j 2.251 \\
0.114 \pm j 2.320\end{array}$ & $\begin{array}{l}-0.443 \\
-0.839\end{array}$ & $\begin{array}{r}-0.0545 \\
0.0220\end{array}$ \\
\hline $\begin{aligned} \mathrm{C}_{\mathrm{n}_{\hat{i}}=}= & 0.0 \\
& \left(\mathrm{rad}^{-1}\right)\end{aligned}$ & $\begin{array}{l}q_{0}=0 \\
q_{0}=12 \mathrm{deg} / \mathrm{sec}\end{array}$ & $\begin{array}{c}-0.0043 \pm j 1.855 \\
0.214 \pm j 1.996\end{array}$ & $\begin{array}{l}-0.499 \\
-1.016\end{array}$ & $\begin{array}{r}-0.127 \\
0.0063\end{array}$ \\
\hline $\begin{aligned} C_{I_{3}}= & -0.338 \\
& \left(\mathrm{rad}^{-1}\right)\end{aligned}$ & $\begin{array}{l}q_{0}=0 \\
q_{0}=12 \mathrm{Geg} / \mathrm{sec}\end{array}$ & $\begin{array}{c}-0.0505 \pm j 1.996 \\
0.150 \pm j 2.638\end{array}$ & $\begin{array}{l}-0.459 \\
-0.904\end{array}$ & $\begin{array}{r}-0.0752 \\
0.0135\end{array}$ \\
\hline
\end{tabular}


The approximations of Eqs. (2.4-2) and (2.4-5)

indicate that the change in the Dutch roll mode's real part should be approximately proportional to the change in $\mathrm{C}_{\mathrm{n}_{\mathrm{r}}}$. Table 3.3-2 indicates that this is not true in this example, for as $C_{n_{r}}$ increases by a factor of 20 , the Dutch roll mode's real part cnly doubles. The other significant effect caused by a veduction in yaw damping magnitude is to destabilize the spiral mode.

TABLE 3.3-2

EFFECTS OF $\mathrm{C}_{\mathrm{n}_{r}}$ VARIATIONS

\begin{tabular}{|c|l|l|l|}
\hline$C_{n_{r}}$ & Dutch Roll & Roll & Spiral \\
\hline \hline-1.200 & $-0.0983 \pm j 2.2468$ & -0.4612 & -0.1396 \\
-0.646 & $-0.0738 \pm j 2.2508$ & -0.4425 & -0.0545 \\
-0.0602 & $-0.0477 \pm j 2.2523$ & -0.4304 & 0.0422 \\
\hline
\end{tabular}

The derivative $C_{n_{\dot{B}}}$ is an acceleration derivative (analogous to $\mathrm{C}_{\mathrm{m}_{\dot{\alpha}}}$ ) that arises because the aerodynamic flow field exhibits some lag in rearranging itself following a change in aerodynamic angle. Acceleration terms are approximations to these flow field dynamics. Experimentally, acceleration derivatives are difficult to measure, and they usually are combined with the rotary derivatives. Analytical studies often make the assumptions that

$$
\begin{aligned}
& \hat{\mathrm{c}}_{\mathrm{n}_{\dot{\beta}}}=0 \\
& \hat{\mathrm{c}}_{\mathrm{n}_{\mathrm{p}}}=c_{\mathrm{n}_{\mathrm{p}}}+\sin \alpha_{0} c_{n_{\dot{\beta}}} \\
& \hat{\mathrm{c}}_{\mathrm{n}_{\mathrm{r}}}=\tau_{\mathrm{n}_{\mathrm{r}}}-\cos \alpha_{0} c_{\mathrm{n}_{\dot{\beta}}}
\end{aligned}
$$


Reference 46 examines some of the possible effects of this approximation on aircraft time histories and parameter identification. It is concluded that significant parameter identification errors can occur if the acceleration derivatives are not included in the model when necessary.

Two types of $c_{n_{\dot{\beta}}}$ effects have been examined. In the first type, non-zero $C_{n_{\dot{\beta}}}$ is added to the model with no other change; this effactively decreases the total damping. In the second type, $C_{n_{p}}$ and $C_{n_{r}}$ are adjusted by subtracting the $C_{n_{\dot{\beta}}}$ terms given in Eqs. $(3.3-8)$ and $(3.3-9)$ to maintain nearly constant damping. In the variable damping case (in which $C_{n_{r}}$ and $C_{n_{p}}$ are constant), the only change is in the Dutch roll eigenvalues. There is significant change in damping and a slight change in frequcncy. This indicates that $C_{n_{\dot{\beta}}}$ primarily affects the Dutch roll mode. The constant damping cases are quite different, in that the changes in $\mathrm{C}_{\mathrm{n}_{\dot{\beta}}}, \mathrm{C}_{\mathrm{n}_{\mathrm{p}}}$, and $\mathrm{C}_{\mathrm{n}_{\mathrm{r}}}$ effectively cancel, as far as the Dutch roll mode is concerned. In this case, however, there are significant changes in the roll and spiral modes. These changes are not due to $C_{n_{\dot{\beta}}}$ (which does not affect the roll and spiral modes) but rather to the corresponding changes in $c_{n_{r}}$ and $c_{n_{p}}$. Therefole, combining $c_{n_{\dot{\beta}}}$ with $C_{n_{p}}$ and $C_{n_{r}}$ leads to erroneous eigenvalue calculations in modes not directly affected by $C_{n_{\dot{\beta}}}$ at all.

Table 3.3-3 illustrates the constant darning rosults with and without steady rolling motion. Non-zero $C_{n_{\dot{\beta}}}$ affects the frequencies of the oscillations only slightly in the presence of rolling motion, but there are significant changes in damping of the various modes. Dutch roll damping ratio changes for non-zero $\mathrm{C}_{n_{B}}$ when the vehicle is rolling, and 
TABLE 3.3-3

EFFECTS OF $c_{n \beta}$ IN THE PRESENCE OF STEADY ROLLING $\left(C_{n_{p}}\right.$ and $C_{n_{r}}$ Adjusted to Yaintain Constant Damping)

$T-0251$

\begin{tabular}{|c|c|c|c|c|c|}
\hline & Short Period & Dutch Roll & no 12 & Splral & Phugoid \\
\hline \multicolumn{6}{|c|}{$c_{n_{\dot{B}}}=0\left(\mathrm{rad}^{-1}\right) \quad p_{W_{0}}=0 \mathrm{deg} / \mathrm{sec}$} \\
\hline & $-0.407 \pm j 1.099$ & $-0.0738 \pm \jmath 2.251$ & -0.443 & -0.0545 & $-0.0 i \epsilon 5=j 0.137$ \\
\hline \multicolumn{6}{|c|}{$c_{n \dot{p}}=0\left(\mathrm{rad}^{-1}\right) \quad p_{W_{0}}=-39.6 \mathrm{der} / \mathrm{sec}$} \\
\hline & $-0.464 \pm J 1.294$ & $-0.0319 \pm j 2 . \approx \div 2$ & \multicolumn{2}{|c|}{$-0.293=j 0.244$} & $0.0427 \pm j 0.189$ \\
\hline \multirow[t]{2}{*}{$\mathrm{C}_{\mathrm{n} \dot{\mathrm{B}}}$} & $=0.55\left(\mathrm{rad}^{-1}\right)$ & $p_{W_{0}}=0 d \in E / \mathrm{se}$ & & & \\
\hline & $-0.407 \pm j 1.099$ & $-0.0757=j 2.256$ & -0.525 & 0.0321 & $-0.0165 \pm j 0.137$ \\
\hline \multirow[t]{2}{*}{$\mathrm{C}_{\mathrm{n} \dot{\mathrm{B}}}$} & $=0.55\left(\mathrm{rad}^{-1}\right)$ & $P_{W_{O}}=-39.6 \mathrm{de}$ & 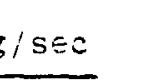 & & \\
\hline & $-0.481 \pm j 1.290$ & $-0.0411 \pm j 2.345$ & \multicolumn{2}{|c|}{$-0.287 \pm j 0.224$} & $-0.0630 \pm j 0.183$ \\
\hline
\end{tabular}

this is not observed for the non-rolling, constant damping case. The conclusion is that an improper $C_{n} \dot{B}$ identification may not appear in the Dutch roll mode for non-rolling flight, but it 8 an caise a significant change in the Dutch roll mode during a rolling maneuver.

The folloving points summarize the findings of this report concerning the effects of lateral stability derivative variations:

- The large amount of rolling in the subject aircraft means that $C_{1}$, dyn and $C_{n_{r} \text { dyn }}$ are poor indicators of the roli mode's eigenvalue and the Dutch roll mode's real part.

- $\quad C_{n_{\beta}}$ and $C_{1_{\beta}}$ variations primarily affect the Dutch roll frequency, as indicated by $C_{n_{B}, d y n}$. Variations due to steady 
pitching motion are independent of the effects caused by changes in $C_{n_{B}}$ and $C_{I_{B}}$.

- Due to the large amount of rolling motion in the Dutch roll mode, $C_{l_{p}}$ has a larger than expected effect on Dutch roll damping, while the effect of $C_{n_{r}}$ is smaller than might be expected.

- $\quad C_{n_{\dot{\beta}}}$ by itseif primarily affects the Dutch roll mode, but subtracting its effect from $C_{n_{p}}$ and $C_{n_{r}}$ (holding the damping constant) leads to large roll mode and spiral mode changes and small Dutch roll mode variations.

- In the presence of steady rolling, there is a significant $C_{n} \dot{\beta}$ effect on the Dutch roll mode even for the constant damping case. Coupled flight conditions should be investignted when the identification of $\mathrm{C}_{\mathrm{n}_{\dot{\beta}}}$ is desired.

\subsection{VARIATIONS DUE TO MASS AND INERTIA EFFECTS}

The variations in the aircraft modes due to mass and rotational inertia changes are examined in this chapter. The rotational inertias considered span the range of aircraft types (from wing-heavy to fuselage-heavy), and the mass variations range from light to heavy wing loadings.

Table 3.4-1 details the aircraft eigenvalue trends as aircraft mass varies. The rotational inertia matrix is held constant (as if a point mass was adder or subtracted at the vehicle's center of gravity), so the ratios between the mass and inertia also vary. The mass change represents a change in relative density, $\mu$, which is defined as

$$
\mu=\mathrm{m} / \rho \mathrm{S} \overline{\mathrm{c}}
$$


TABLE $3.4-1$

EFFECTS OF AIRCRAFT MASS ON EIGENVALUE LOCATION

$\left(v_{0}=94 \mathrm{~m} / \mathrm{s}, \alpha_{0}=15 \mathrm{deg}\right)$

\begin{tabular}{|c|c|c|c|c|c|}
\hline Mass & Short Period & Phugo1d & Dutch Roll & Roll & Spiral \\
\hline $3060 . \mathrm{kg}$ & $-0.572 \pm j 1.07$ & $-0.028 \pm j 0.186$ & $-0.120 \pm j 2.25$ & -0.442 & -0.054 \\
$5055 . \mathrm{kg}$ & $-0.442 \pm j 1.09$ & $-0.019 \pm j 0.150$ & $-0.084 \pm j 2.25$ & -0.442 & -0.055 \\
$8175 \mathrm{~kg}$ & $-0.367 \pm j 1.10$ & $-0.013 \pm j 0.120$ & $-0.062 \pm j 2.25$ & -0.443 & -0.055 \\
\hline
\end{tabular}

where $\bar{c}$ is 9 reference length. The relative density relates the aircraft mass to the air density, and therefore indicates the relative magnitude of aerodynamic and inertial effects.

Variations of the aircraft mass have a large effect on the damping of the rotational oscillations -- Dutch roll mode and short. period mode -- but only a negligible effect on their frequencies. The spiral and roll modes also are essentially unchanged. "ass variations change the phugoid mode's natural frequency and damping ratio, because this mode involves the interchange of kinetic and potential energy and is highly mass dependent.

The rotational inertia of the aircraft describes the distribution of the aircraft mass about the center of gravity. Most of the inertia is due to the fuselage and the wing, and the relation between them leads to the designation of a specific configuration as "wing-heavy" or "fuselage-heavy."

The yaw inertia is approximately 10 to 15 percent larger than the pitch inertia, and the roll inertia can be from 4 to 12 times smaller than the yaw inertia. High performance fighters emphasize $r_{i g h}$ rolling performance, and tend to be fuselage-heary. Transport aircraft are built for cruising officiency and tond to be wing-heary. 
Table 3.4-2 details the results of two rotational inertia investigations, one with constant rolling inertia $\left(I_{x}\right.$ ) and varying fuselage inertia ( $I_{y}$ and $I_{z}$ ), and one with constant fuselage inertia and varying rolling inertia. In neither of these casf - is the phugoid mode significantly affected, underlining the conclusion that the phugoid is a translational mode rather than a rotational mode. For the uncoupled reference flight condition, the short period mode is affected only by a change in pitch inertia: an increase in pitch inertia causes a major decrease in short period frequency and a small decrease in damping ratio.

TABLE 3.4-2

EFFECT OF ROTATIONAL INERTIA ON EIGENVALUES

\begin{tabular}{|c|c|c|c|c|c|c|}
\hline \multicolumn{2}{|c|}{ Fuselage } & \multicolumn{5}{|c|}{ Inertia Varied $-I_{X}$ Held Constant $\left(V_{0}=81 \mathrm{~m} / \mathrm{s}, \alpha_{0}=25 \mathrm{deg}\right)$} \\
\hline$I_{z} / I_{x}$ & $I_{y} / I_{x}$ & Short Period & Dutch RoII & Roll & Spiral & Phugoid \\
\hline 4.4 & 4.0 & $-0.534 \pm \mathrm{J} 2.01$ & $0.102 \pm j 1.59$ & -0.483 & -0.071 & $-0.018 \pm 0.156$ \\
\hline 8.8 & 8.0 & $-0.329 \pm j 1.43$ & $0.085 \pm j 1.54$ & -0.366 & -0.050 & $-0.018 \pm j 0.157$ \\
\hline 13.2 & 12.0 & $-0.261 \pm \mathrm{J} 1.17$ & $0.0788 \pm j 1.52$ & -0.327 & -0.039 & $-0.017=j 0.157$ \\
\hline
\end{tabular}

\begin{tabular}{|c|c|c|c|c|c|c|}
\hline \multicolumn{6}{|c|}{ Roll Inertia Varied $-I_{y}$ and $I_{z}$ Held Consiant $\left(V_{0}=94 \mathrm{~m} / \mathrm{s}, \alpha_{0}=15 \mathrm{def}\right)$} \\
\hline$I_{z} / I_{x}$ & $I_{y} / I_{x}$ & Short Perlod & Dutch Roll & Roll & Spiral & Phusoid \\
\hline 4.4 & 4.0 & $-0.407 \pm j 1.099$ & $-0.0428 \pm j 1.82 .6$ & -0.313 & -0.0587 & $-0.0165 \pm j 0.137$ \\
8.8 & 8.0 & $-0.407 \pm j 1.099$ & $-0.0738 \pm j 2.251$ & -0.443 & -0.0545 & $-0.0165=j 0.137$ \\
\hline
\end{tabular}

The lateral modes are affected by an increase in rolling inertia, in that both Dutch roll frequency and roll mode response are slowed significantly. There is a significant decrease in Dutch roll damping and very little change in the spiral mode. Larger values of yaw inextia lead to somewhat different effects. Both roll and spiral modes are significantly slower, and there is sore decrease in Dutch roll damping ratio and irequency. 
The effects of a large rolling inertia on the rolling/slipping stability are shown in Fig. 3.4-1. The stability boundaries are generally similar to those shown in Fig. 2.4-10, although they differ in detail. The Dutch roll mode is stable for the higher rolling inertia. The phugoid instability combines with an unstable spiral mode at high sideslip angles and roll rates to form a fast, highly unstable oscillation.

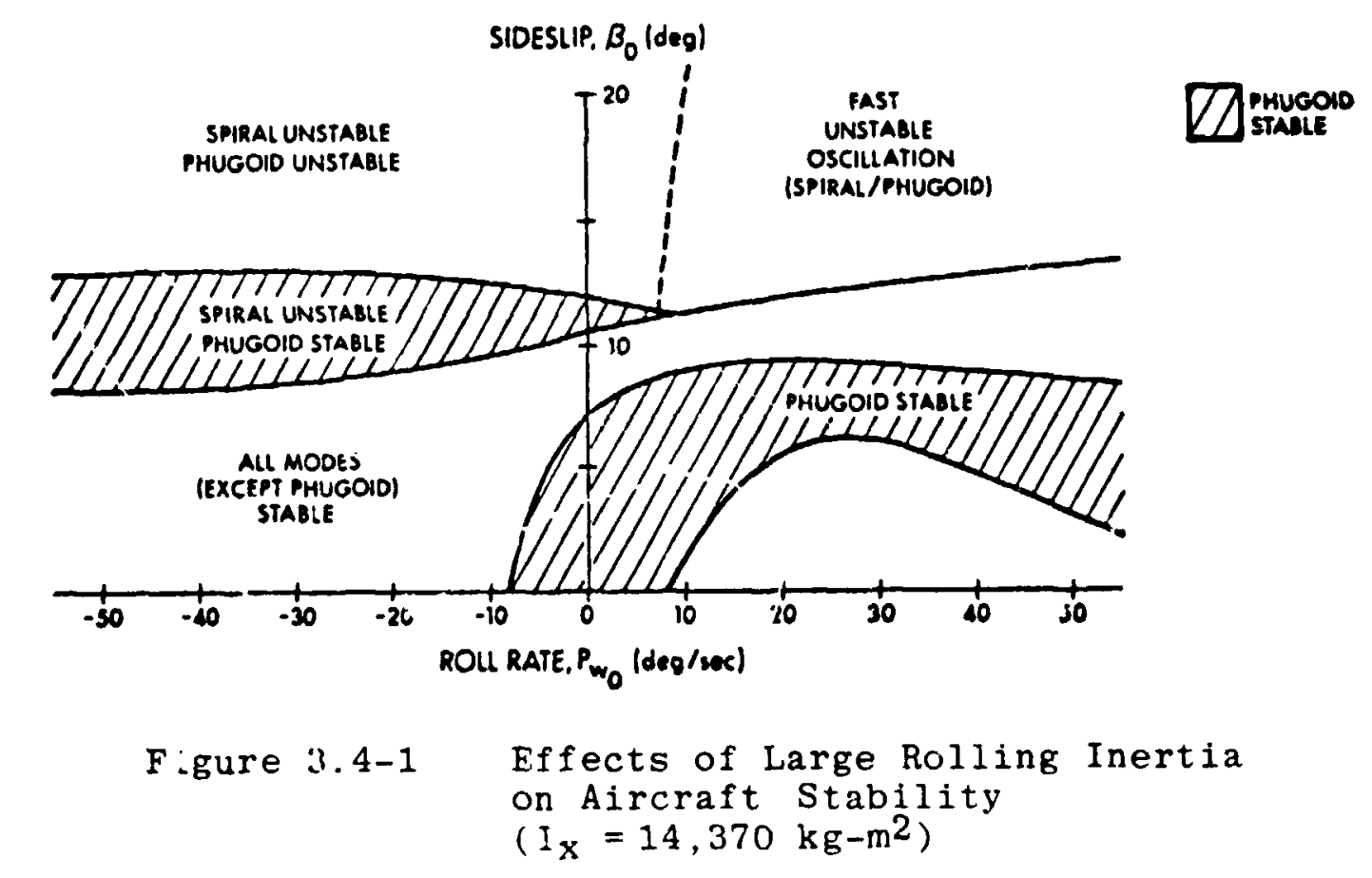

Conclusions concerning the effects of mass and jnertia variations are suinmarized as follows:

- Mass increases raduce Dutch roll and short period damping, but mass variations do nct have large effect on the short period or Dutch roll mode frequencies. They io not cause significant changes in the roll or spiral modes. 
- The phugoid mode is a translational mode and is greatly affected by mass variations. The phugoid eigenvalue does not depend strongly on rotational inertia.

- Increases in pitch inertia reduce short period frequency and damping.

- Increases in roll inertia increase the roll mode time constant, decrease the Dutch roll frequency significantly, ard modify the effects of mode coupling due to asymmetric flight.

- Increases in yaw inertia primarily slow the roll and spiral modes, and there is some effect on Dutch roll damping and frequency.

\subsection{CLASSIFICATION OF DEPARTURES}

Departure from controlled flight can occur in two ways. Unforced departures are due to instabilities in the basic aircraft. Even if the pilot does not move the controls, sinall perturbations in the aircraft states build up until the aircraft can no longer be controlled. In a forced departure, the basic aircraft may or may not be unstable, but the addition of a pilot loop closure creates an unstable vehiclepilot system. The two following sections discuss these departure classes.

\subsubsection{Unforced Departure ludes}

Unforced departures occur when the pilot cannot or dofs not stabilize an unstable vehicle. The vehicle eigenvalues directly indicate the open-loop system stability in this case, so that iany of the stability boundaries that have been shown in this report can be classed as unforced departure boundaries. 
The speed of the fast modes (Dutch roll mode, short period mode and roll mode) is such that the stability of these modes is critical, and the least stable of these usually is the Dutch roll mode. This mode can become unstable in two ways, either resulting from negative "spring terms" or resulting from negative damping, both of which can he influenced by aerodynamics and coupling effects.

The approximate equations for the Dutch roll mode (Eqs. (2.4-1) and (2.4-2)) indicate a pure static instability for large, negative $C_{n_{B}}$, but the exact result is somewhat more complex. As in the case of longitudinal mode coupling due to positive $\mathrm{C}_{\alpha}$ (Section 3.2), directional instability can cause the Dutch roll mode to couple with the classical roll and spiral modes, and it can lead to a new oscillatory mode, analogous to the so-called "roll-spiral" or "lateral-phugoid" mode. Figlre 3.5-1 illustrates a case in which negative $\mathrm{C}_{n_{B}}$ causes an oscillatory mode that has low natural frequency and is highly unstable. By comparison to the conventional Dutch roll mode (Fig. 2.4-3), there is a significant change in mocle shape. There

SPIRAL

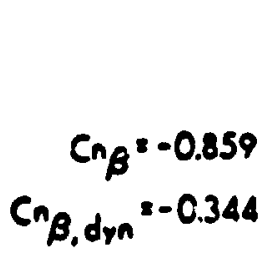

ROLL-SPIRAL

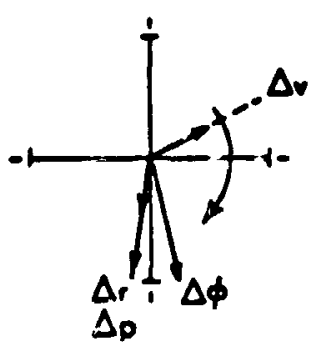

$0.755 \pm j 0.620$

ROLL

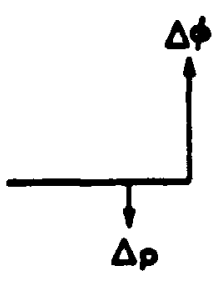

$-0.334$

Figure 3.5-1 An Example of Lateral-Directional Eigenvalurs for Negative Directional Stability 
is a 180-deg phase change in the yaw-rate component, as well as a substantial roll angle change. In addition, the spiral mode has gained significant $\Delta v, \Delta r$, and $\Delta p$ components.

The deparuure caused by this type of instability is shown in Fig. 3.5-2. Although the linear nodel indicates that this motior is an osciilatinn: it is so unstable that only part of a period appears on the. we history plot. The first few seconds of the motion exhibit a rapid roll-yaw angular motion. The pilot would sense a rapid rotational divergence about this axis and might refer to it as a rolling "nose slice" or yaw departure.
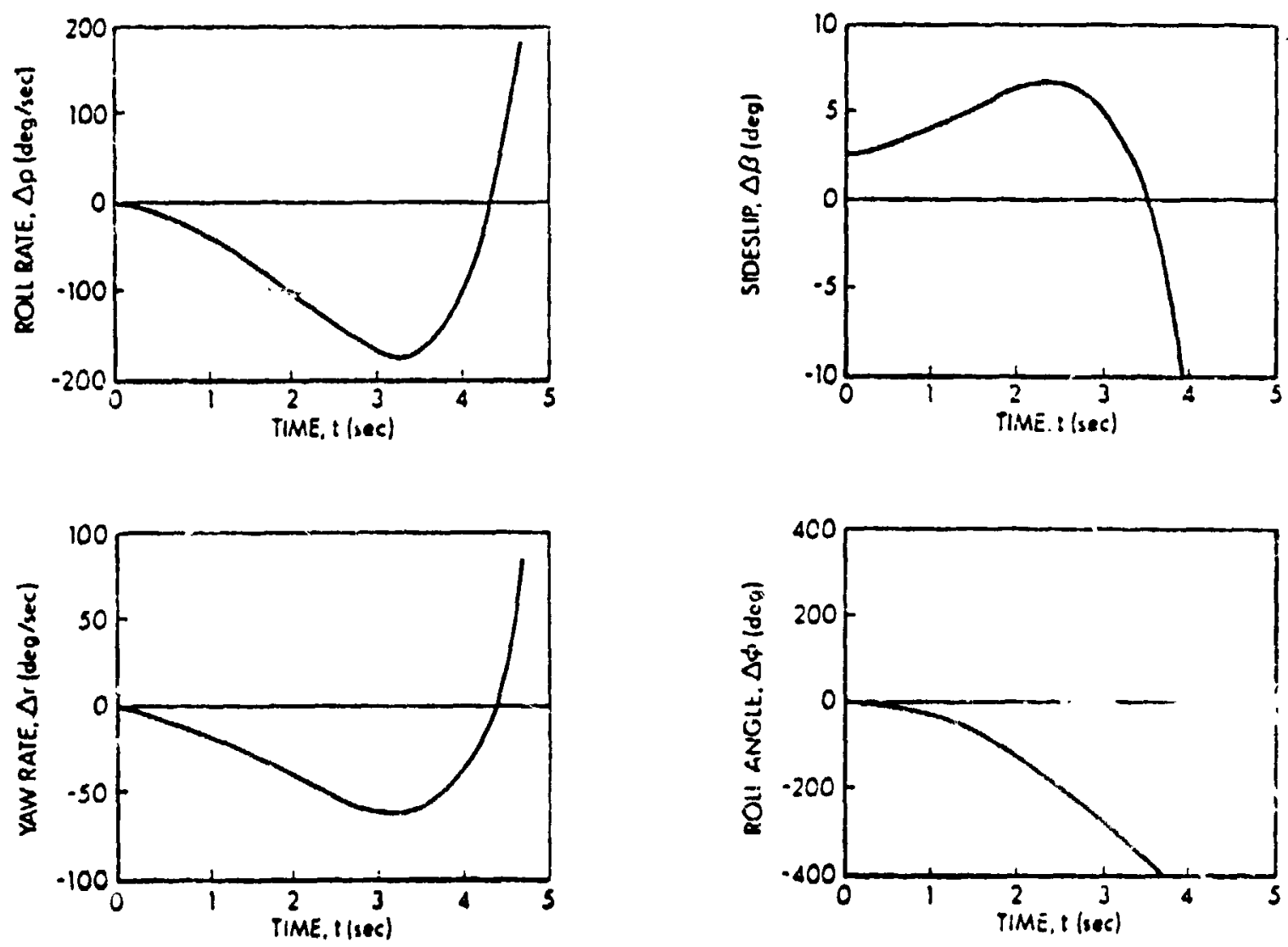

Figure 3.5-2 An Unforced Depasture Due to Negative $C_{n_{B}}$ 
Negative yaw damping leads to a more conventional destabilizing of the putch roll mude, which retains its characteristic mode shape. The time hiscory of a departure due to dynamic Dutch roll instability is shorn in Fig. 3.5-3, and the difference in shape from the departure due to static instability is apparent.
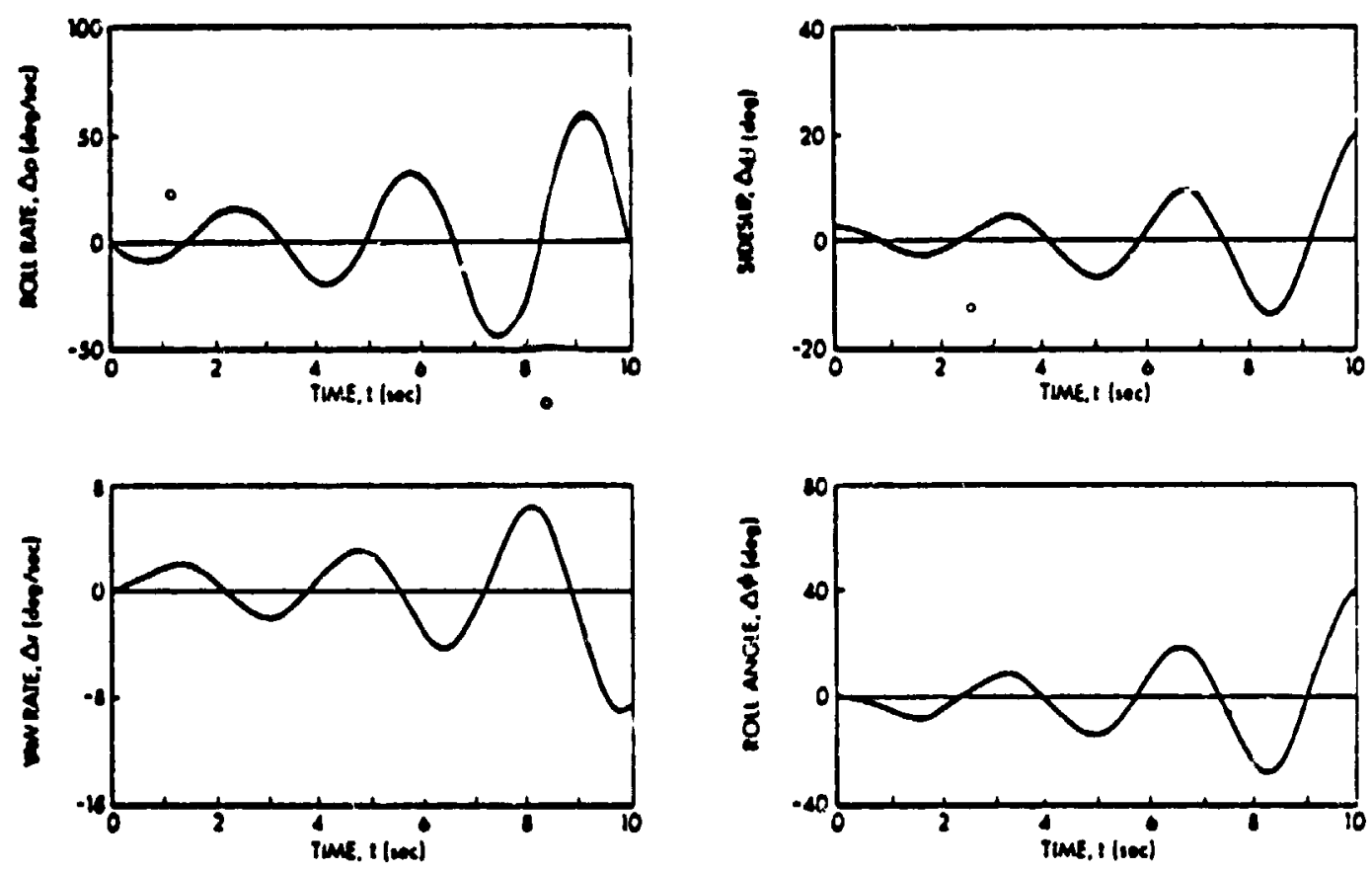

Figure 3.5-3 An Unforced Departure Due to Negative Dutch Roll Damping

The large amount of rolling motion in the Dutch roll mode indicates that this may be what pilots refer to as "wing rock." This is uncertain, however, since "wing rock" also could be a roll-spiral oscillation or a limit cycle caused by an aerodynamic non-linearity. In any case, a pilot sensing such an rsciliation probably would unload t'e aircraft by reducing the angle of attack, removing the aircraft from tra region of instability. 
A third type of unforced departure can occur at high sideslip angles. The modes of motion al shown in Fig. 3.5-4, $a_{\text {. }}$ the unstable roll roode is seen to exhibit a mixed rollingyawing departure characteristic. This divergence is rapid and, as shown in the stability boundari s of Fig. 2.4-2, can appear with cnly small aerodynamic angle changes from a much more benign flight condition.
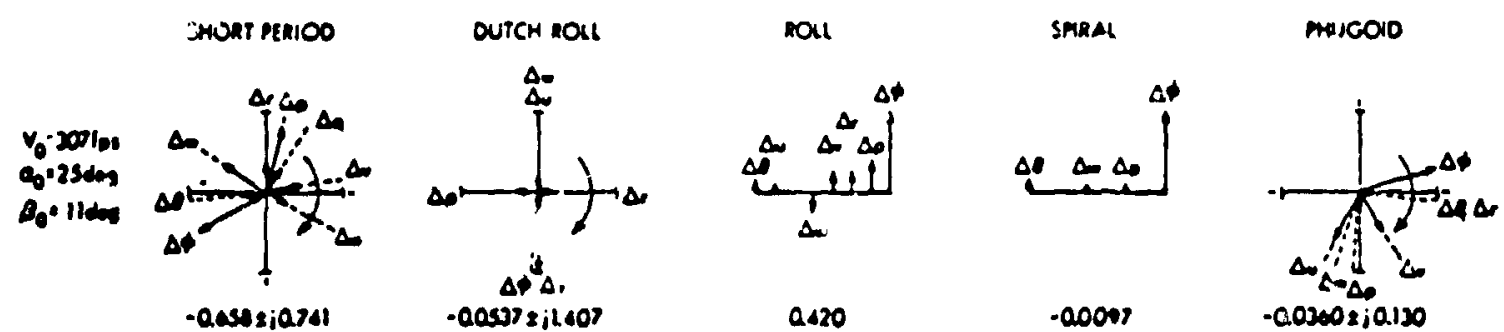

Figure 3.5-4 Eigenvalues and Eigenvectors for a Flight Condition with Large Sideslip Angle

This unstable roll mode is essentialiy a pure roll about the stability $x$-axis, but, because of the large nominal aerodynamic angles, it appears as a roll-yiw motion in body axes. It is possible that a pilot fiying an aircraft at these large angles uuld interpret a siability-axis rolling depa ture as a "nose slice."

\subsubsection{Forced Departure Mudes}

Control inputs fiom a pijot or control system can force an aircraft to depart from controlled flight in two ways. In the first way, Averse responee to pilot inpuis moves the nominal flight condition into an unstable region where an unforced departure can occur. The second iussible cause of a forced departure is an improper loop closure that creates an unstable closed-loop sy'stem. Departuro prevention procedures are quite different for the two cascs; in 
the former case, positive control action is necessary for recovery, whi'e a neutralization of control inputs might allow a recovery from the latter departure.

An example of an improper loop closure was presented in Section 2.6. In that case, the target-tracking pitc: attitude-to-elevator loop closure caused lateral mode stability problems at coupled flight conditions. This underlines the necessity of including control cross-couplings in situations where the system itself is coupled.

The target-tracking example can be considered as a situation in which the pilot learns to control the aircraft at one flight condition but does not change his control strategy as the flight condition changes. This is emphasized by the observation that coupled flight conditions often exhibit drastic changes in the shapes of the normal modes, so that a pilot might apply the wrong control action.

The investigation of Section 2.5 demonstrates that control effectiveness problems may leave the pilot no alternative but to apply a poor control combination. For example, a: 25-deg angle of attack, the roll moment due to aileron is essentially zero. The rudder would rave to be used for roll control but this brings an unavoidable sideslip response with it. This sideslip could drive the vehicle into the roll divergence region cited in the last section.

As an exarple of unexpected control response, Fig. 3.5-5 shows a departure caused by an aileron input. Normally, the result would be a significant negative roll rate, but the sideslip and yaw rate build up so rapidly that the instability of the basic aircra't results in a rapid roling departure with positive roll rate. 

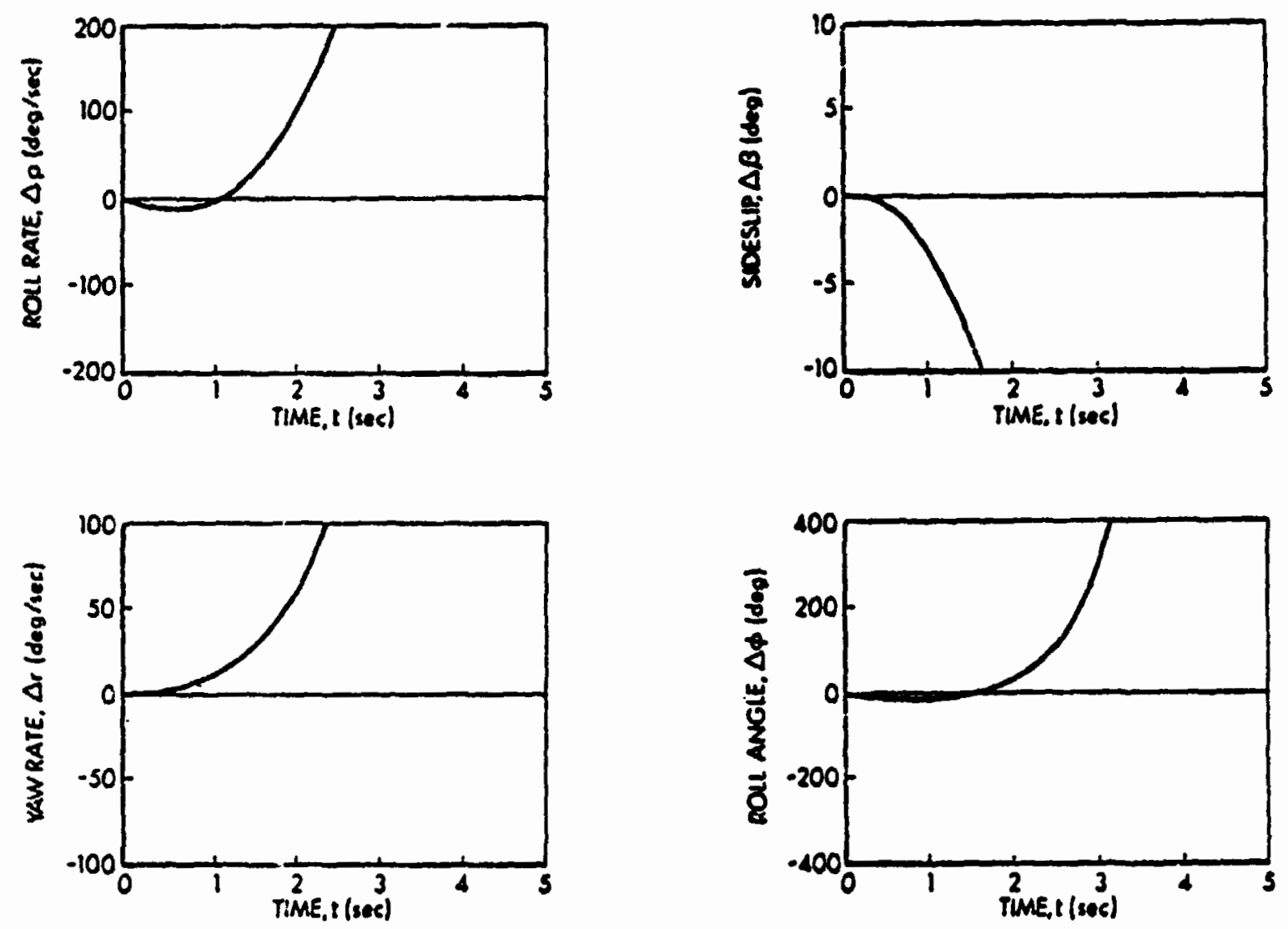

Figure 3.5-5 Aileron Input for Negative $C_{n_{B}}$

Situations in which improper pilot inputs are likely are discussed in Section 2.5. These situations are characterIzed by reduced stability of the open-loop system (due to high angle of attack or pitch rate) and highly coupled modes caused by an asymmetric flight condition (such as non-zero $\beta_{0}$ or $\mathrm{p}_{\mathrm{W}_{0}}$ ). Noruminimum-phase zeros of ten appear and can cause great difficulty if "tight" control is attempted.

The conclusions regarding departure modes are summarized as follows: 
- An unforced departure (one due to an unstable open-loop system) is most likely to appear in the lateral oscillatory mode. Static instability results in a rapid rolling-yawing departure, while dynamic instability causes an unstable "wing rock" motion.

- An unstable wind-axis roll divergence can appear at extreme aerodynamic angles.

- Forced departures can occur when degraded control response causes the pilot to fly the aircraft into a flight condition where unforced departures are likely.

- Mode coupling or unexpected nonminimumphase zeros can change the control response so that a "normal" control loop closure leads to an unstable closed-loop system.

\subsection{CHAPTER SUMMARY}

This chapter has presented effects of configurational variations on aircraft dynamics. Relationships between mode approximations and exact results are discussed for longitudinal, lateral, and coupled motions, and examples of various departure types are presented. It is shown that the effects of aerodynamic parameter variations are modified by the coupling which results in asymmetric flight, particularly in regard to the transfer of damping (due to rotary derivatives) from longitudinal to lateral-directional modes (and vice-versa). Time histories of linearizedmodel response illustrate departure characteristics similar to those experienced in flight. 


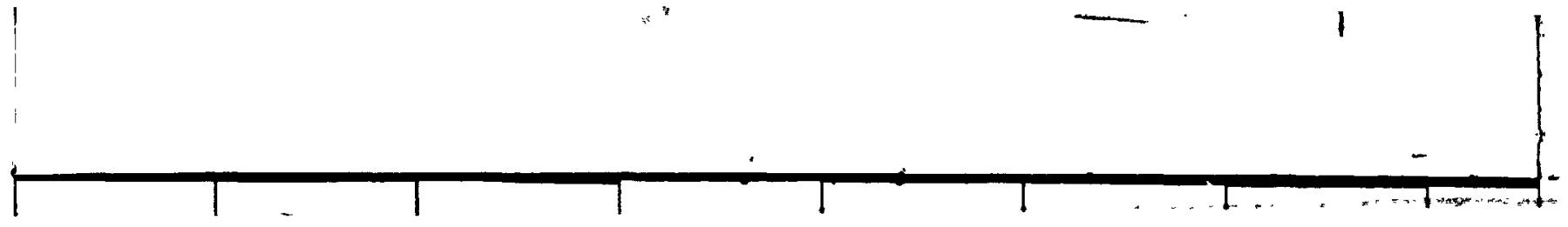

\section{PREVENTION OF DEPARTURE FROM CONTROLLED FLIGHT}

\subsection{OVERVIEW}

As indicated by earlier developments in this report and the summary of prior work in Section 2.2, there is ample reason to consider designing stability augmentation systems for the specific purpose of preventing departure. Aircraft design is dominated by performance requirements, and even unconstrained configuration modifications may not provide adequate stability or control response (especially during extreme maneuvering). Apperdix $A$ and Chapters 2 and 3 demonstrate how linear-time-invariant models of aircraft dynamics can be derived for studying stability and control response during difficult maneuvers. These models are used to illustrate stability augmentation system concepts in the present chapter.

Unlike earlier studies of departure prevention, the powerful tools of linear-optimal control theory are applied to the problem in this chapter. Since new grourd is broken and methods which are unfamiliar (in the departure prevention context) are presented, the objective is to provide preliminary guidelines for Departure-Prevention Stability Augmentation System (DPSAS) development. Therefore, a simple optimal controller -- the continuous-time linearoptimal regulator -- is applied to departure prevention. A linear-optimal regulator is a feedback control law of the form.

$$
\Delta \underline{u}(t)=-K \Delta \underline{x}(t)
$$


where $\Delta \underline{u}(t)$ is the vector of control command perturbations, $\Delta \underline{x}(t)$ represents the vectc: of the aircraft's dynamic states, and $K$ is the gain matrix which scales the state measurements for proper stabilization ar 1 compensation of the aircraft's motion. (An equivalent discrete-time linear-optimal regulator, for which the state is measured and control is commanded at a fixed sampling interval, car be derived for a digital flight control system.) This control law has several qualities which are desirable for the present study, in which $i$ is assumed that system dynamics are known exactly and that all states are measured precisely:

\footnotetext{
- The control gains guarantee stability of the closed-loop system.

- Complete longitudinal/lateral-directional coupling is assumed and is accounted for in the design process.

- The control design technique identifies all significant crossfeeds and interconnects, as well as feedback gains.

- Tradeoffs between the amplitudes of state perturbations and of control motions are specified in the design process.
}

In addition, a gain-scheduling algorithm which accounts for varying maneuver conditions is developed.

The control design techniquiss applied to the DPSAS can be generalized to full command augmentation systems for a high-performance aircraft. Referince 58 shows how practical command-response control laws can be developed for a highly coupled aircraft, a tandem-rotor helicopter. These control laws satisfy classical step-response criteria, adapt to flight condition, honor rate- and displacement-limits on control actuators, and use incomplete (possibly noisy) 
feedback measurements. A command-response system for a high performance fighter is described in Ref. 59. It adapts to flight condition to provide uniform handling qualities throughout the flight regime. These control laws are developed for direct implementation in a digital computer and use low sampling rates. This extension of the DPSAS to. a complete flight control system, while promising, is a subject for future study.

The remainder of this chapter is directed to a brief explanation of linear-optimal regulator design and extensive application of this control design approach to DPSAS examples. Section 4.2 presents the linear-optimal regulator and a discussion of the parameters used in computing control gains. Control designs are developed for a reference aircraft over a wide range of angles of attack, pitch rates, sideslip angles, and roll rates at a single altitudevelocity point -- the central flight condition of $6100 \mathrm{~m}$, $94 \mathrm{~m} / \mathrm{s}$-- in Section 4.3. The symmetric and asymmetric variations in flight condition are considered separately, in order to make the differentiation between pullup and sldeslip-rolling effects more apparent. Control gains are computed at 32 maneuvering conditions to obtain the results of Section 4.3; with eight states fed back to four control effectors, over 1000 gains are generated. In Section 4.4, these gains are correlated with each other and with maneuver conditions to identify candidate interconnects and gainscheduling relationships. Negligible and constint gains also are identified in the process. The chapter is summarized in Section 4.5 . 


\subsection{THE LINEAR-OPTIMAL REGULATOR}

Optimal control theory provides a useful and practical multi-input, multi-output control system design tool. Linear-optimal control methods are based on the differential equations that describe the vehicle in the time domain (Eq. (A.3-3)), and they produce feedback controllers which exhibit desirable properties.

The problem is to find a controller for the system described by Eq. (A.3-3), which exhibits a linear feedback structure (Eq. (4.1-1)) and minimizes a scalar-valued cost functional of the state and the control:

$$
J=\int_{0}^{\infty}\left(\Delta \underline{x}^{T} Q \Delta \underline{x}+\Delta \underline{u}^{T} R \underline{u}\right) d t
$$

This controller is called a linear-optimal regulator, and it is derived in Refs. 60 to 62 .

The designer's freedom rests in his choice of the weighting watrices, $Q$ and $R$. The design procedure consists of the choice of $Q$ and $R$, the coinputation of the Riccati matrix, an evaluation of closed-loop performance, and the adjustment of $Q$ and $R$ as discussed in Section A.4.4.

The linear-optimal regulator is a tool for designIng a Departure-Frevention Stability Augmentation System (DPSAS). It is not a limiter, because no limits are placed on the pilot's control authority, and it is not an automatic spin-recovery system, because open-loop anti-spin control settings are not implemented. The DPSAS is intended to augment stability and to minimize the gyrations which precede loss of pilot control. The DPSAS makes full use of 
avallable control power, and, in this respect, could compete with the pilot's control commands; however, the designer can specify the amount of control-surface displacement which normally is available to the DPSAS. Basing the system on the linear-optimal regulator, the DPSAS can be designed to use less than full control authority for expected magnitudes of aircraft maneuvers, leaving a percentage of control authority free for manual commands.

The primary objective of this chapter is to Identify the basic effects of varying fljght condition on the structure of a DPSAS. To keep the number of varying parameters to a minimum in this demonstration, the state and control weighting factors (Eq. (A.4-21) and (A.4-22)) are chosen at a single symmetric flight condition and held constant throughout the sweep of 32 maneuvering conditions. $Q$ and $R$ elements which provide satisfactory eigenvalues, acceptable time response, and reasonable control gains are chosen at the central flight condition of this sweep $\left(\alpha_{0}=15 \mathrm{deg}, V=94 \mathrm{~m} / \mathrm{s}\right.$ fps, $\left.H=6100 \mathrm{~m}\right)$. Thus, it is expected that eigenvalues and control gains will vary with flight condition, but the rms-values of state and control perturbations should remain relatively constant.

The $Q$ and $R$ elements are used as design parameters which can be interpreted as the following maximum allowable rms perturbations:

$\begin{array}{lll}\text { - } & \text { Throttle setting: } & 100 \% \text { of full scale } \\ \text { - } & \text { Elevator deflection: } 20 \mathrm{deg} \\ \text { - } & \text { Alleron deflection: } & 60 \mathrm{deg} \\ \text { - } & \text { Rudder deflection: } & 30 \mathrm{deg} \\ \text { - } & \text { Euler angle: } & 30 \mathrm{deg} \\ \text { - Body angular rate: } & 25 \mathrm{deg} / \mathrm{sec} \\ \text { - Body velocity: } & 9 \mathrm{~m} / \mathrm{s}\end{array}$




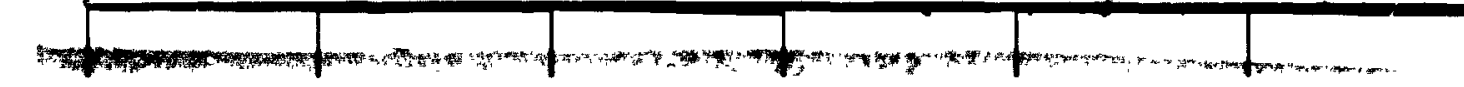

These values indicate that throttle setting, elevator, aileron, and rudder are allowed to vary between their limits and that angles of attack and sideslip must be held within $5.6 \mathrm{deg}$ (the $9 \mathrm{~m} / \mathrm{s}$ body velocities correspond to aerodynamic angles of this number). Table 4.2-1 indicates that the primary effects of the loop closures at the central flight condition are to increase short period, Dutch roll, and phugoid damping and to quicken the roll and spiral modes.

TABLE $4.2 \cdots 1$

EFFECTS OF DPSAS AT THE CENTRAL FLIGHT CONDITION

\begin{tabular}{|c|c|c|c|c|c|c|}
\hline \multirow[b]{2}{*}{$\begin{array}{c}\text { Dynamic } \\
\text { Mode }\end{array}$} & \multicolumn{3}{|c|}{ Open-Lonp Characteristics } & \multicolumn{3}{|c|}{ Closed-Loop Characteristice } \\
\hline & $\begin{array}{l}\text { Natural } \\
\text { Frequency, } \\
\text { rad/sec }\end{array}$ & $\begin{array}{c}\text { Danpina } \\
\text { Ratio. } \\
\text { - }\end{array}$ & $\begin{array}{l}\text { Time } \\
\text { Constant, } \\
\text { sec }\end{array}$ & $\begin{array}{l}\text { Natural } \\
\text { grcquency, } \\
\text { rad/sce }\end{array}$ & $\begin{array}{l}\text { Damping } \\
\text { Ratio, } \\
\text { - }\end{array}$ & $\begin{array}{c}\text { Time } \\
\text { Const2nt, } \\
\text { sec }\end{array}$ \\
\hline Short Perlod & 1.17 & 0.35 & - & 2.67 & 0.72 & - \\
\hline Dutch Ro12 & 2.25 & 0.03 & - & 2.35 & 0.69 & - \\
\hline Ro11 & - & - & 2.26 & - & - & 0.36 \\
\hline Spiraj & - & - & 18.34 & - & - & 1.07 \\
\hline Phurold & 0.14 & 0.12 & - & 0.16 & 0.99 & - \\
\hline
\end{tabular}

This illustraies implicitly that the linear-optimal regulator design can prodice stricter tracking than indicated by the choice of $Q$ and $R$ elements. The $\Delta \alpha$ and $\Delta \beta$ requirements can be met only by increasing damping and decreasing time constants. This infers that Euler angles and body angular rates also are closely regulated, even though the weighting of the corresponding elenents in $Q$ is light. Table 4.2-1 also indicates that the selection of equal weights on $\Delta v$ and $\Delta w$ (and, therefore, on $\Delta \alpha$ and $\Delta \beta$ ) drives the natural frequencies and damping ratios of the short period and Dutch roll to similar values. 
The DPSAS gain matrix for this flight condition is listed in Table 4.2-2. The gain matrix illustrates why damping is increased in the closed-loop system; rate feedbacks are large. The classical longitudinal/lateral-directional partition can be observed in the gains; the control algorithm actually computes coupling gains on the order of 10-7 due to the use of single-precision arithmetic. These gains can be ignored. The elevator is seen to be the primary longitudiral controller, as throttle feedback gains are smail (the principal effect of throttle control is to damp the phugoid mode). Lateral-directional control largely partitions along the roll and yaw axes. Although the gains shown in Table 4.2-2 have reasonable magnitudes, they could be reduced by reducing the values of $\mathrm{q}_{i \mathrm{i}}(\mathrm{Eq} \cdot(\mathrm{A} .4-21))$. Transient response would be altered, but the system would remain stable.

TABLE $4.2-2$

DPSAS GAIN MATRIX AT THE CENTRAL FLIGHT CONDITION

\begin{tabular}{|c|c|c|c|c|c|c|c|c|}
\hline Control Ovtput & $\begin{array}{l}\text { Pitch } \\
\text { Angle. } \\
\text { deg }\end{array}$ & $\begin{array}{c}\text { Body } x-2 \times 1 \\
\text { Velocity. } \\
m / 8\end{array}$ & $\begin{array}{c}\text { Pitcb } \\
\text { Rate. } \\
\text { deg/sec }\end{array}$ & $\begin{array}{c}\text { Body z-exts } \\
\text { Veivelty. } \\
\text { m/a }\end{array}$ & $\begin{array}{c}\text { Body } y-2 \times 1 \\
\text { Volocity. } \\
m /=\end{array}$ & $\begin{array}{c}\text { Yaw } \\
\text { Rate. } \\
\text { der / } / \text { aec }\end{array}$ & $\begin{array}{r}\text { Roll } \\
\text { Rate, } \\
\text { deg/sec }\end{array}$ & $\begin{array}{l}\text { Roll } \\
\text { Angle, } \\
\text { deg }\end{array}$ \\
\hline $\begin{array}{l}\text { Throttle sottias. } \\
\text { Fraction of ruli } \\
\text { Scale }\end{array}$ & -0.010 & 0.069 & -0.005 & 0.020 & 0 & o & 0 & 0 \\
\hline $\begin{array}{l}\text { Elevetor Ancle, } \\
\text { des }\end{array}$ & -0.700 & 1.322 & -1.540 & -0.988 & 0 & 0 & 0 & 0 \\
\hline $\begin{array}{l}\text { Alleros Angle, } \\
\text { des }\end{array}$ & 0 & 0 & 0 & 0 & -0.500 & 0.138 & 2.840 & 1.804 \\
\hline $\begin{array}{l}\text { Euddor Aesle, } \\
\text { des }\end{array}$ & 0 & 0 & 0 & 0 & 1.042 & -3.000 & 0.228 & -0.100 \\
\hline
\end{tabular}

The performance of the linear-optimal regulator is demonstrated by comparing open- and closed-loop response to perturbations in angle of attack, sideslip angle, and roll rate. Figure 4.2-1 illustrates that a 1.1-deg $\Delta_{\alpha}$ perturbation is moderately damped without the regulator al.d well-damped with 


\section{1}

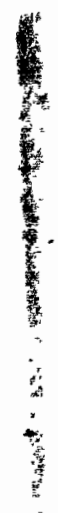
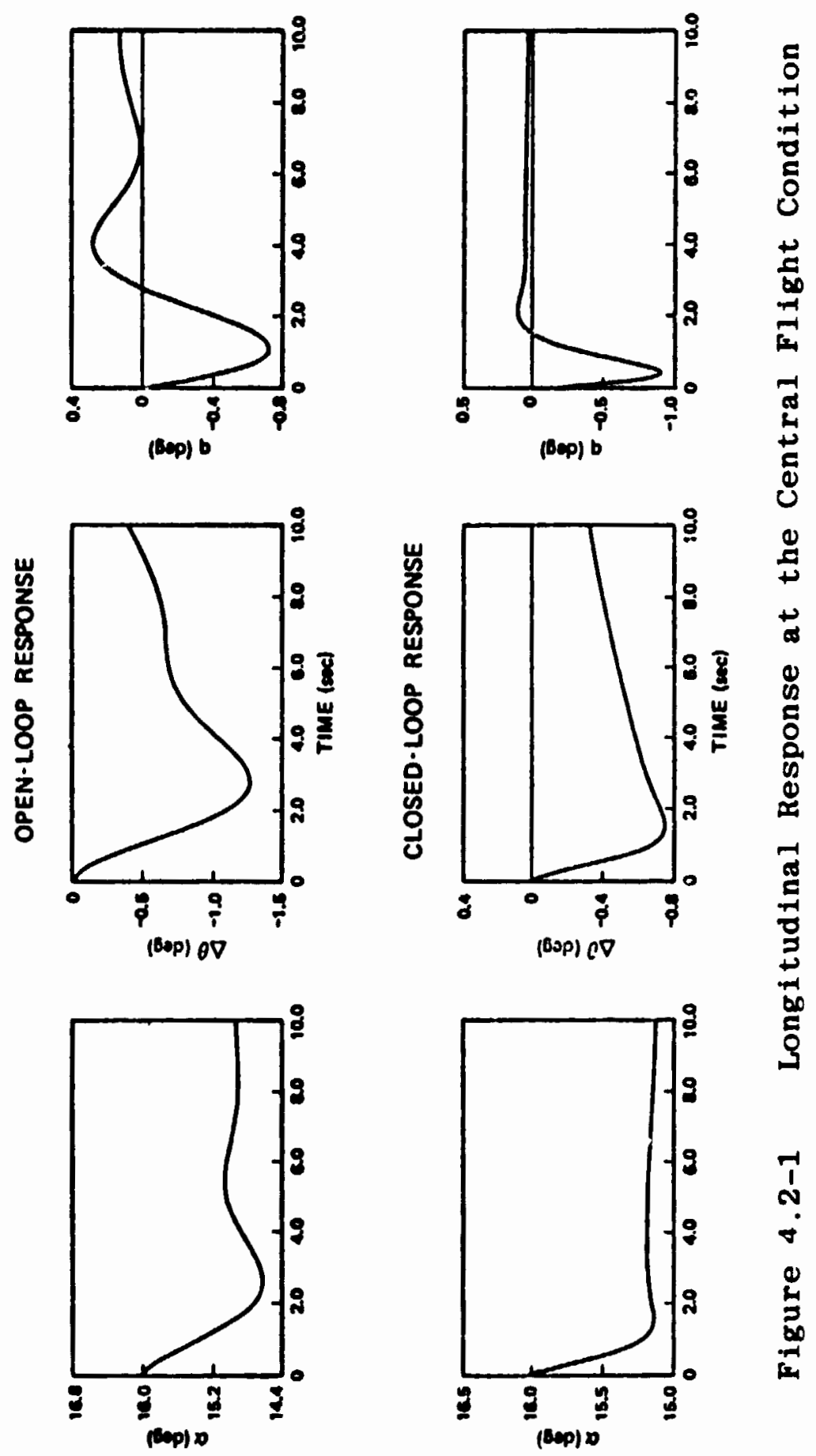
the regulator. Figure 4.2-2 shows that the lightly damped natural motion resulting from a 1 -deg $\Delta B$ initial condition creates a substantial amount of roll as well as yaw. The regulator damps the oscillation and limits the roll angle excursion to 20 percent of its open-loop value, providing significant decoupling of lateral and directional motions. This decoupling effect is confirmed by the aircraft's closedloop response to a roll-rate disturbance of $1 \mathrm{deg} / \mathrm{sec}$ ( $\mathrm{Fig}$ ure 4.2-3). This initial condition creates a small sideslip oscillation and triggers the spiral node (indicated by the underlying exponential response trend in roll angle). The regulator damps the oscillation, reduces the sideslip response by 70 percent, and stabilizes the roll angle.

Having obtained a representative design point for the DPSAS at the central flight condition, the effects of maneuvering on control gains, aircraft stability, and time response are examined in the next section.

\subsection{DPSAS CONTROL LAWS}

The control gains obtained at the central flight condition would stabilize the aircraft for some range of nominal angles and angular rates; however, changes in the aircraft's dynamics (reflected by variations in $F$ and $G$ ) would lead to less-than-optimal regulation. It is necessary, therefore, to redesign the control gain matrix at each maneuvering condition in order to assess the full possibilities for preventing departure with the inearoptimal control laiv.

Two separate maneuvering condition sweeps have been conducted, using the reference aircraft flying at $6100 \mathrm{~m}$ 
霎
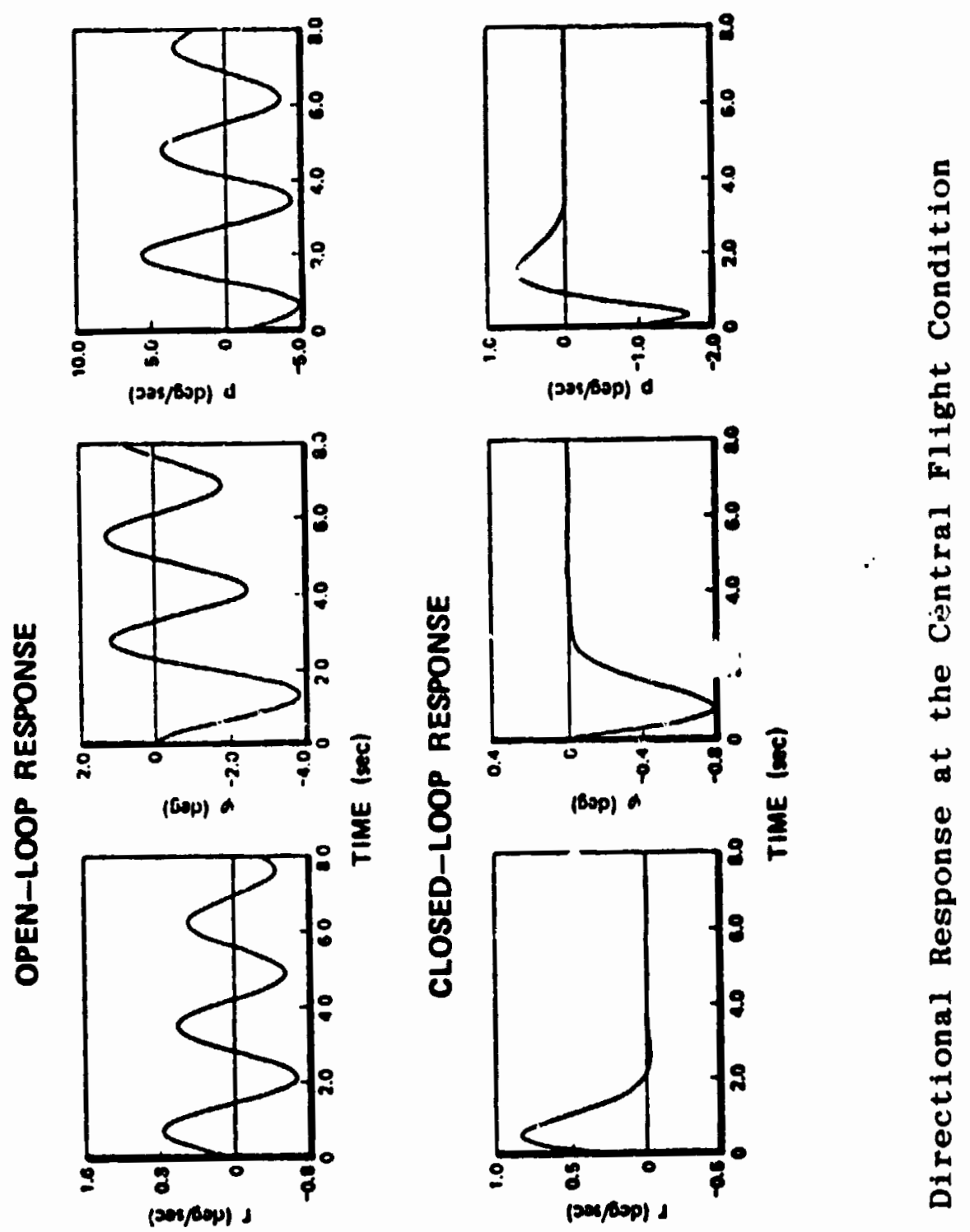

동

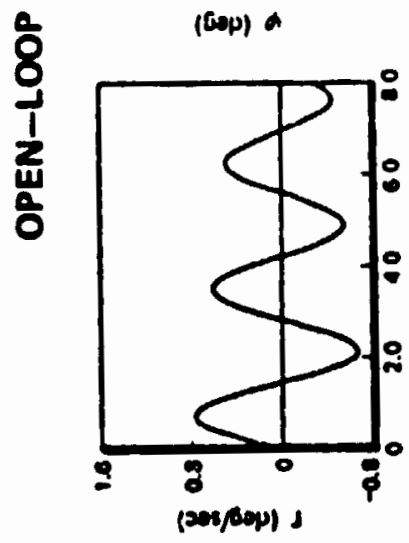

崖
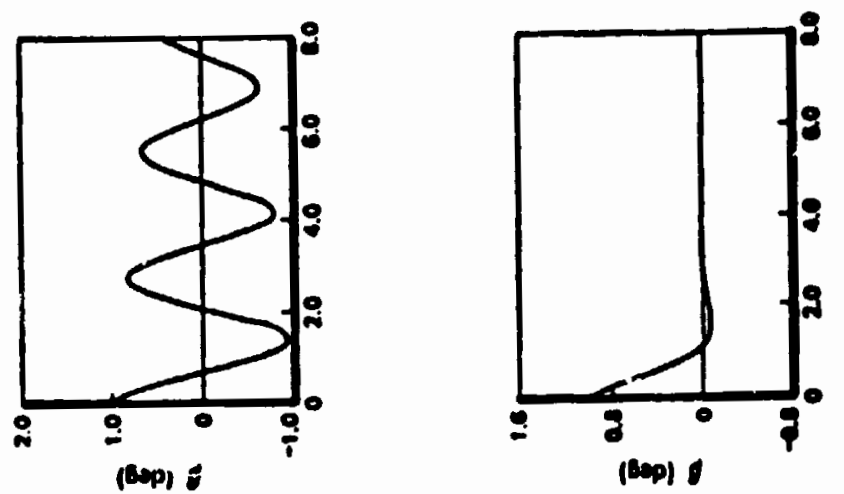

9
1
0
0
0
0
0
0 

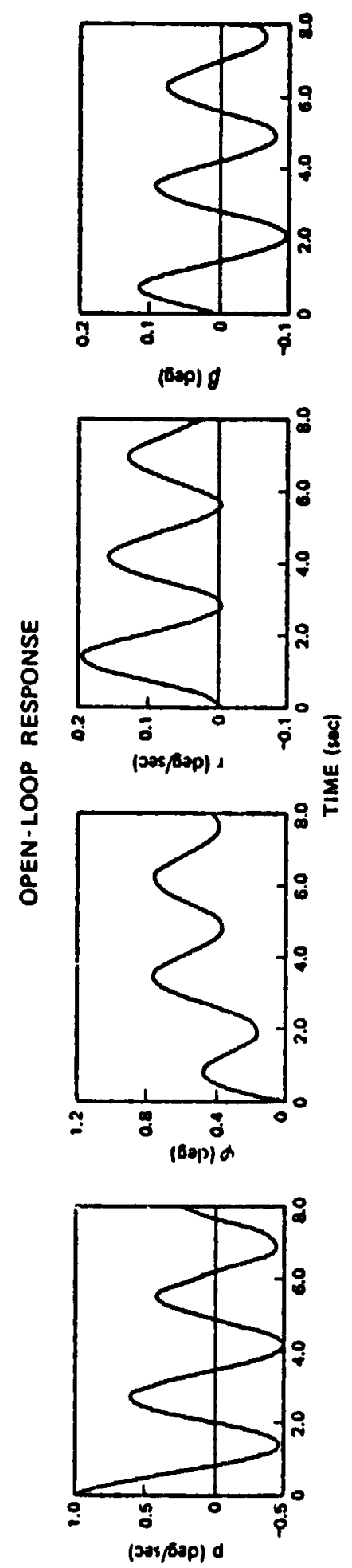
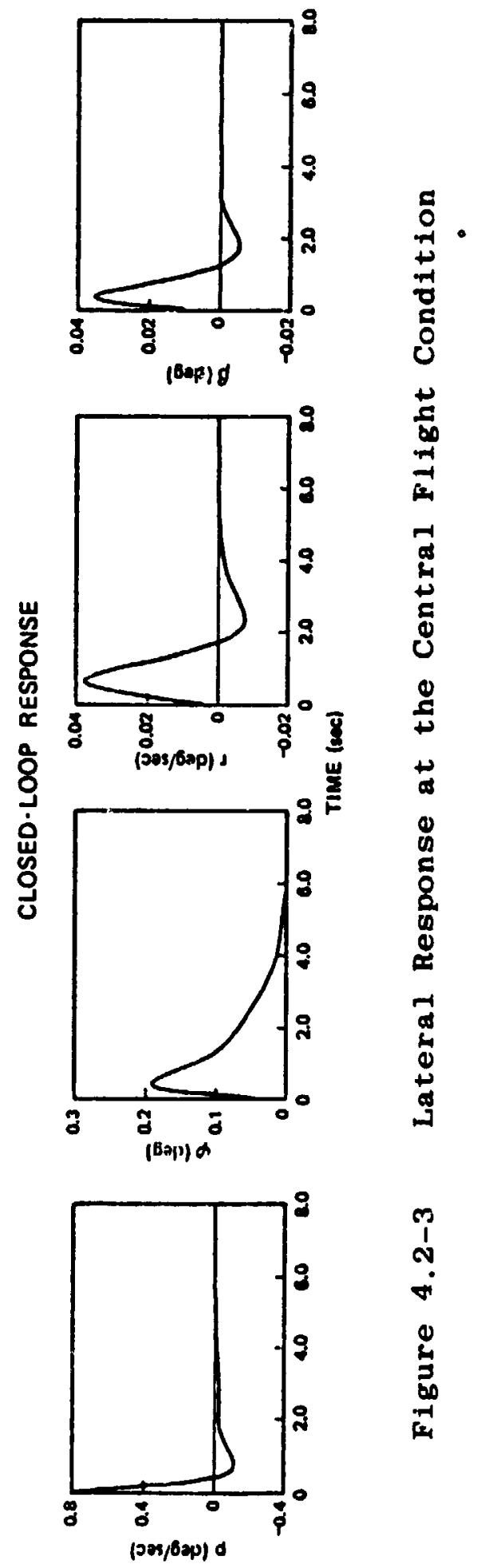

Closed-loop stability at the 15 conditions is summarized by Table 4.3-1, where it can be seen that all modes are stable and at least moderately damped. Control power does not change with $q_{0}$, but it does change with $\alpha_{0}$; consequently, the closed-loop stability at a given $\alpha_{0}$ is relatively independent of $q_{0}$. There is a gradual decrease in Dutch roll damping as $\alpha_{0}$ increases, and roll response becomes more sluggish. This happens because rudder and aileron are less effective at the higher angles, while the elements of $R$ which weight the cost of using these surfaces remain unchanged. A heavily damped roll-spiral oscillatory mode occurs at $\alpha_{0}$ of 20 and $25 \mathrm{deg}$. A coupled roll-spiral mode can degrade handling qualities, so adjustment of

TABLE . 4 . 3-1

CLOSED-LOOP STABILITY IN THE LONGITUDINAL SWEEP

\begin{tabular}{|c|c|c|c|c|c|c|c|c|c|}
\hline \multicolumn{2}{|c|}{$\begin{array}{l}\text { Maneuver } \\
\text { Condition }\end{array}$} & \multicolumn{2}{|c|}{ Short Period } & \multicolumn{2}{|c|}{ Duteh Roll } & \multirow{2}{*}{$\frac{\text { Roll }}{\mathrm{sec}}$} & \multirow{2}{*}{$\frac{\text { Spiral }}{\text { sé }}$} & \multicolumn{2}{|c|}{ Phugoid } \\
\hline $\begin{array}{l}\text { a } \\
\text { deg }\end{array}$ & $\begin{array}{c}90 \\
\text { deg/sec }\end{array}$ & $\begin{array}{c}\omega_{n} \cdot \\
\mathrm{rad} / \mathrm{sec}\end{array}$ & 5 & $\begin{array}{c}\omega_{n} \cdot \\
\mathrm{rad} / \mathrm{sec}\end{array}$ & 5 & & & $\begin{array}{c}\omega_{\mathrm{n}} \cdot \\
\mathrm{rad} / \mathrm{sec}\end{array}$ & $\underline{5}$ \\
\hline \multirow[t]{3}{*}{3} & 0 & 2.43 & 0.71 & 1.90 & 0.52 & 0.14 & 1.18 & $5.54^{\circ}$ & $8.06^{\circ}$ \\
\hline & 12 & 2.43 & 0.69 & 1.93 & 0.53 & 0.14 & 1.23 &. $.43^{\circ}$ & $16.82^{\circ}$ \\
\hline & 24 & 2.44 & 0.67 & 2.02 & 0.55 & 0.14 & 1.32 & $.65^{\circ}$ & $18.31^{\circ}$ \\
\hline \multirow[t]{3}{*}{10} & 0 & 2.60 & 0.72 & 1.91 & 0.57 & 0.17 & 1.44 & 0.17 & 0.95 \\
\hline & 12 & 2.60 & 0.71 & 1.99 & 0.58 & 0.17 & 1.20 & $2.36^{\circ}$ & $14.95^{\circ}$ \\
\hline & 24 & 2.60 & 0.69 & 2.08 & 0.39 & 0.17 & 1.30 & $1.60^{\circ}$ & $17.23^{\circ}$ \\
\hline \multirow[t]{3}{*}{13} & 0 & 2.67 & 0.72 & 2.35 & 0.69 & 0.36 & 1.07 & 0.16 & 0.99 \\
\hline & 12 & 2.67 & 0.71 & 2.42 & 0.67 & 0.37 & 1.17 & $2.31^{\circ}$ & $28.23^{\circ}$ \\
\hline & 24 & 2.68 & 0.70 & 2.51 & 0.66 & 0.34 & 1.30 & $1.57^{*}$ & $21.25^{\circ}$ \\
\hline \multirow[t]{3}{*}{20} & 0 & 2.72 & 0.72 & 2.95 & 0.57 & $1.08^{*}$ & $0.97^{\dagger}$ & 0.16 & 0.88 \\
\hline & 12 & 2.72 & 0.72 & 2.97 & 0.56 & 0.82 & 1.06 & $2.22^{\circ}$ & $28.19^{\circ}$ \\
\hline & 24 & 2.72 & 0.71 & 3.00 & 0.55 & 0.86 & 1.34 & $1.50^{\circ}$ & $20.21^{\circ}$ \\
\hline \multirow[t]{3}{*}{23} & 0 & 2.75 & 0.62 & 2.22 & 0.40 & $0.80^{\dagger}$ & $0.90^{\dagger}$ & $6.09^{\circ}$ & $9.58^{\circ}$ \\
\hline & 12 & 2.78 & 0.61 & 2.23 & 0.38 & $0.79^{+}$ & $0.93^{\dagger}$ & $2.57^{\circ}$ & $67.57^{\circ}$ \\
\hline & 24 & 2.81 & 0.60 & 2.25 & 0.39 & $0.78^{+}$ & $0.89^{+}$ & $1.79^{\circ}$ & $58.41^{\circ}$ \\
\hline
\end{tabular}

12 Real Rsots

troll-Spiral

and 6 
$Q$ and $R$ could be necessary to eliminate this characteristic.

The normally oscillatory phugoid mode degenerates into two real modes at most maneuvering conditions considered here. The over-damped phugoid mode may result from the "cost" associated with $\Delta$ u perturbations, which could be relaxed in future DPSAS designs.

There are 16 non-trivial DPSAS gains generated for the pullup maneuver. Scheduling of these gains is discussed in Section 4.4, and 12 of the gains (four each for elevator, aileron, and rudder) are presented here. Table 4.3-2 lists these gains for $\alpha_{0}$ of 5,15 , and $25 \mathrm{deg}$ and $q_{0}$ of $0, .12$, and $24 \mathrm{deg} / \mathrm{sec}$. The first subscript of $\mathrm{k}$ indicates the control effector (in the order used in Table 4.2-2) and the second subscript indicates the feedback variable (also ordered in Table 4.2-2).

Longitudinal Gains ( $k_{21}$ to $k_{24}$ ) - The gains maintain an orderly progression with both $\alpha_{0}$ and $q_{0}$; none change sign and most follow a single increasing or decreasing trend with the iwo flight variables. Table 4.3-2 shows that the pitch-rate gain $\left(k_{23}\right)$ is dominant at all maneuver conditions and has a maximum variation of less than 25 percent, which is representative of the variations of most gains at most conditions.

Directional Gains ( $k_{45}-$ to $_{4}$ ) - Substantial variations in rudder gains can be expected with increasing $\alpha_{0}$. The fuselage blocks the flow over the vertical tail at high $\alpha_{0}$, and the rudder side force transforms into stability axis roll and yaw moments differently at different angles of attack. Unlike the longitudinal galns, there is a dramatic change in the directional gains as $\alpha_{0}$ increases from 15 to 


\begin{tabular}{|c|c|c|c|c|c|c|c|}
\hline & $\frac{8}{4}$ & 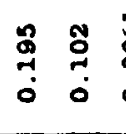 & & $\begin{array}{ll}8 & 8 \\
0 & 0 \\
0 & 0 \\
1 & 1\end{array}$ & 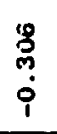 & $\begin{array}{l}\overrightarrow{0} \\
m \\
\dot{p} \\
i\end{array}$ & 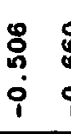 \\
\hline & & 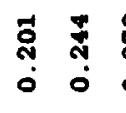 & 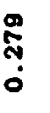 & 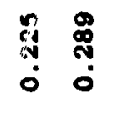 & $\stackrel{7}{m}$ & \begin{tabular}{l}
$\stackrel{8}{\circ}:$ \\
\hdashline \\
0
\end{tabular} & $\begin{array}{ll}\stackrel{0}{0} & 8 \\
0 & 8 \\
0 & \end{array}$ \\
\hline & $\underbrace{4}_{8} x^{9}$ & 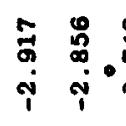 & & 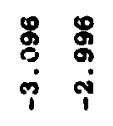 & $\begin{array}{l}\stackrel{.}{\infty} \\
\stackrel{\infty}{0} \\
\text { ì }\end{array}$ & : & $\begin{array}{l}5 \\
\text { in: } \\
1\end{array}$ \\
\hline & $\frac{z}{y^{n}} x^{n}$ & 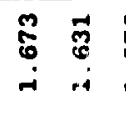 & مُ & 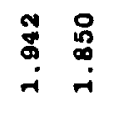 & $\stackrel{8}{\stackrel{8}{\circ}}$ & $\underset{0}{0}$ & 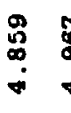 \\
\hline 曼 & $\sum_{i}^{\infty}$ & 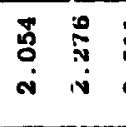 & $\begin{array}{l}\overrightarrow{7} \\
\text { in } \\
\text { in }\end{array}$ & 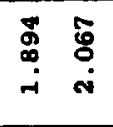 & 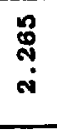 & $\begin{array}{l}\stackrel{g}{p}: \\
\stackrel{p}{0}:\end{array}$ & $\underset{7}{7}$ \\
\hline 莟品 & $\sum_{\substack{\infty \\
\infty}}^{\infty}$ & 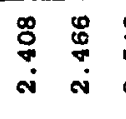 & 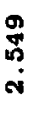 & $\begin{array}{ll}0 & 0 \\
\vdots & 0 \\
i & 0 \\
i & 0\end{array}$ & $\underset{\vec{i}}{\vec{a}}$ & $\underset{+}{\overrightarrow{0}}$ & $\begin{array}{l}0 \\
:\end{array}$ \\
\hline 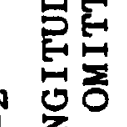 & $\underbrace{\infty}_{\substack{\infty \\
0}}$ & 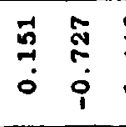 & בְ & 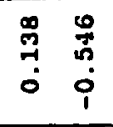 & $\begin{array}{l}8 \\
i \\
i\end{array}$ & $\begin{array}{l}\stackrel{8}{\%}: \\
\stackrel{\text { i }}{0}\end{array}$ & 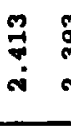 \\
\hline 里恣 & $\underbrace{3}_{8}$ & 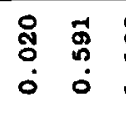 & $\begin{array}{l}9 \\
\vdots \\
-1 \\
-i\end{array}$ & 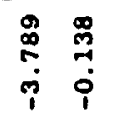 & $\begin{array}{l}n \\
\dot{n} \\
0\end{array}$ & 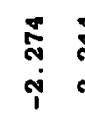 & 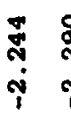 \\
\hline 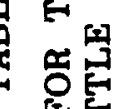 & $\sum_{\infty}^{5}$ & 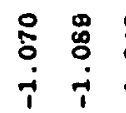 & 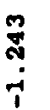 & $\begin{array}{ll}0 & 0 \\
0 & 0 \\
0 & 0 \\
0 & 0\end{array}$ & $\stackrel{8}{\circ}$ & $\stackrel{\leftrightarrow}{\stackrel{a}{i}}$ & 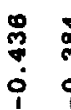 \\
\hline 总胥 & $\sum_{0}^{g}$ & 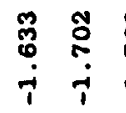 & i & 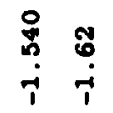 & $\begin{array}{l}8 \\
0 \\
-i\end{array}$ & $\stackrel{8}{i}$ & 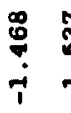 \\
\hline \& & $\sum_{0}^{2}$ & 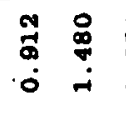 & $\begin{array}{l}\text { i⿱ } \\
6 \\
\text { - }\end{array}$ & 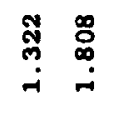 & 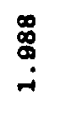 & ঙ̊: & 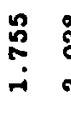 \\
\hline & $\frac{9}{4}{ }_{0}^{9}$ & $\begin{array}{ll}\overrightarrow{\hat{\sigma}} & \overrightarrow{\overrightarrow{0}} \\
\dot{0} & \dot{0} \\
\mathbf{i} & \mathbf{1}\end{array}$ & $\begin{array}{l}\text { o } \\
\$ \\
\infty \\
\dot{0} \\
i\end{array}$ & 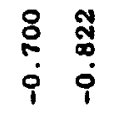 & \begin{tabular}{l}
0 \\
\multirow{2}{\infty}{} \\
0 \\
0 \\
$i$
\end{tabular} & $\begin{array}{l}\stackrel{8}{8} \\
\stackrel{\rho}{0} \\
i\end{array}$ & $\begin{array}{ll}\overrightarrow{1} & 8 \\
0 & \vdots \\
\dot{0} & 8\end{array}$ \\
\hline & $\sigma^{\circ} \frac{\mathscr{d}}{\not}$ & $0 \cong$ & $\nexists \tilde{\pi}$ & 0 & $\bar{N}$ & 0 & 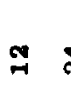 \\
\hline & $\dot{0}$ & $n$ & & 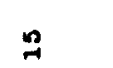 & & $\stackrel{s}{s}$ & \\
\hline
\end{tabular}


$25 \mathrm{deg}$ (Table 4.3-2). The yaw gains $\left(\mathrm{k}_{45}\right.$ and $\left.\mathrm{k}_{46}\right)$ have significant changes with both $\alpha_{0}$ and $q_{0}$. The roll-angla gain $\left(k_{48}\right)$ is noted to change sign as $\alpha_{0}$ progresses from 5 to $15 \mathrm{deg}$, while the increased roll-rate gain $\left(\mathrm{k}_{47}\right)$ attempts to provide stability-axis yaw damping.

\section{Latera! Gains $\left(\mathrm{k}_{35}\right.$ to $\left.\mathrm{k}_{38}\right)$ - Trends in the aileron}

gains also have large variation with $\alpha_{0}$ due to geometric transformation, loss of rudder effectiveness, and aileron yaw effects. Mort gains change sign, and pitch rate has a greater effect on rain magnitude. There is an abrupt reduction in the use of aileron for roll control $\left(k_{37}\right.$ and $\left.k_{38}\right)$ at an $\alpha_{0}$ of $25 \mathrm{deg}$, which is accompanied by increased aileron use for yaw control $\left(\mathrm{k}_{35}\right.$ and $\left.\mathrm{k}_{36}\right)$.

It was noted earlier that pitch rate destabilizes the Dutch roll, roll, and spiral modes. The coupled nature of this phenomenon has an interesting effect on the secondary lateral-directional control paths, i.e., the yaw feedback to the roll moment controller (and the converse), such as $k_{35}$, $\mathrm{k}_{36}, \mathrm{k}_{47}$, and $\mathrm{k}_{48}$ at the lower angles of attack. These gains have as great or greater variation with $q_{0}$ as with the change from 5- to $15-\mathrm{deg} \alpha_{0}$, which is not the case for the primary control paths (yaw-to-rudder and roll-to-dileron).

As in the previous section, the performance of the DPSAS in the pullup flight condition is assessed oy comparing open- and closed-loop time responses. Figuie 4.3-1 illustrates the aircraft's open- and closed-lcop responses to an initial sideslip perturbation when $\alpha_{0}$ is $15 \mathrm{deg}$ and $\mathrm{q}_{0}$ is $12 \mathrm{deg} / \mathrm{sec}$. The oscillation grows at a moderate rate without stability augmentation but is damped in one cycle with the control loops closed. At higher angle of attack (25 deg) and the same pitch rate. the open-loop oscillation 

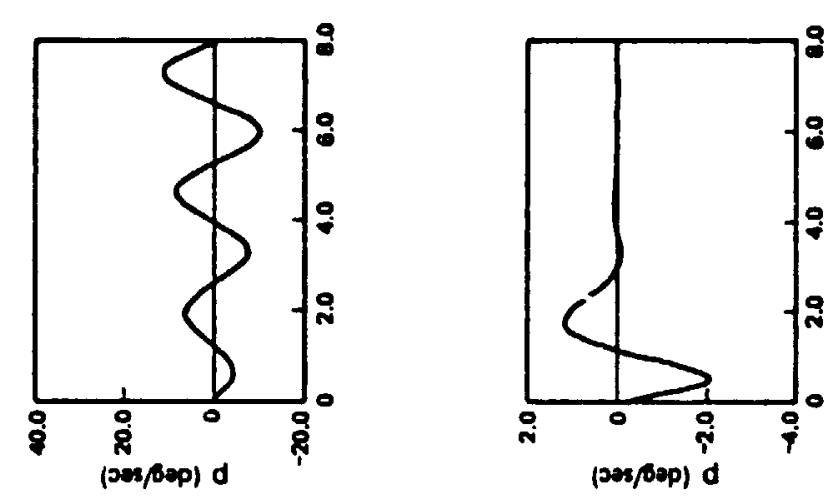

0
0
8
0
0
0
0
0
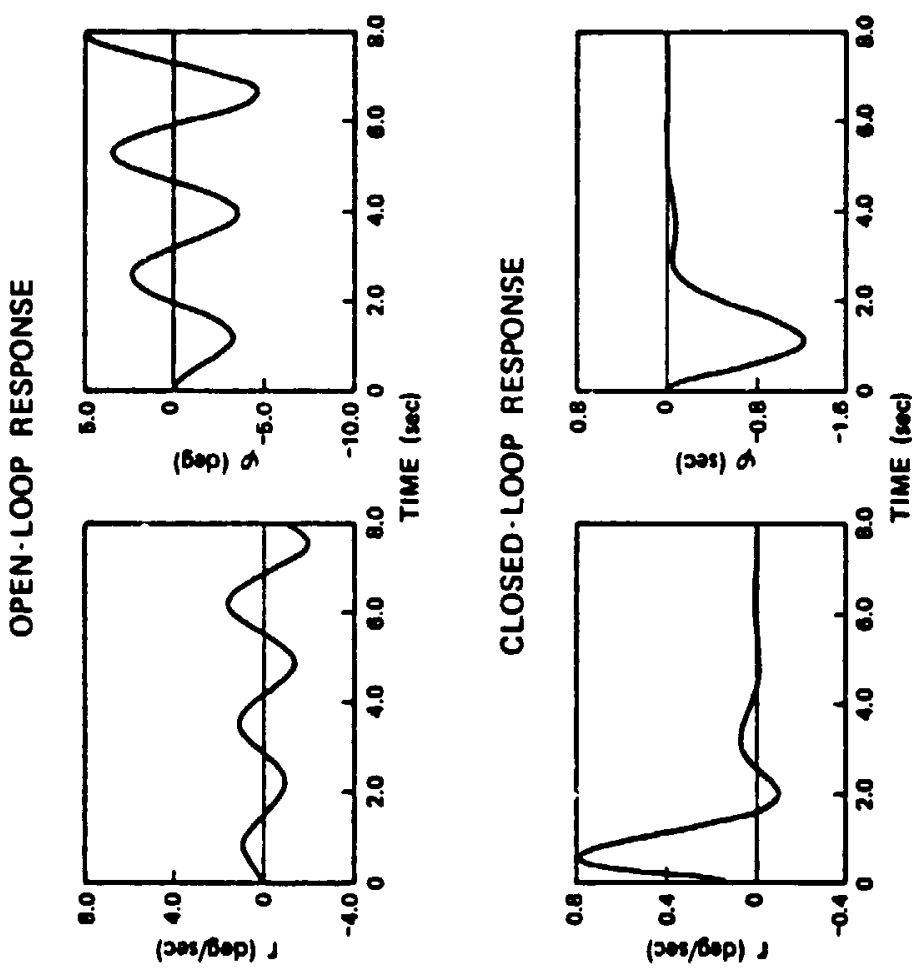

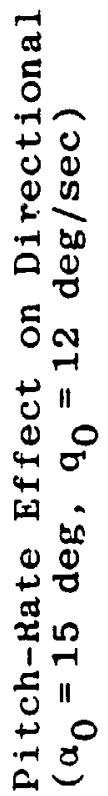
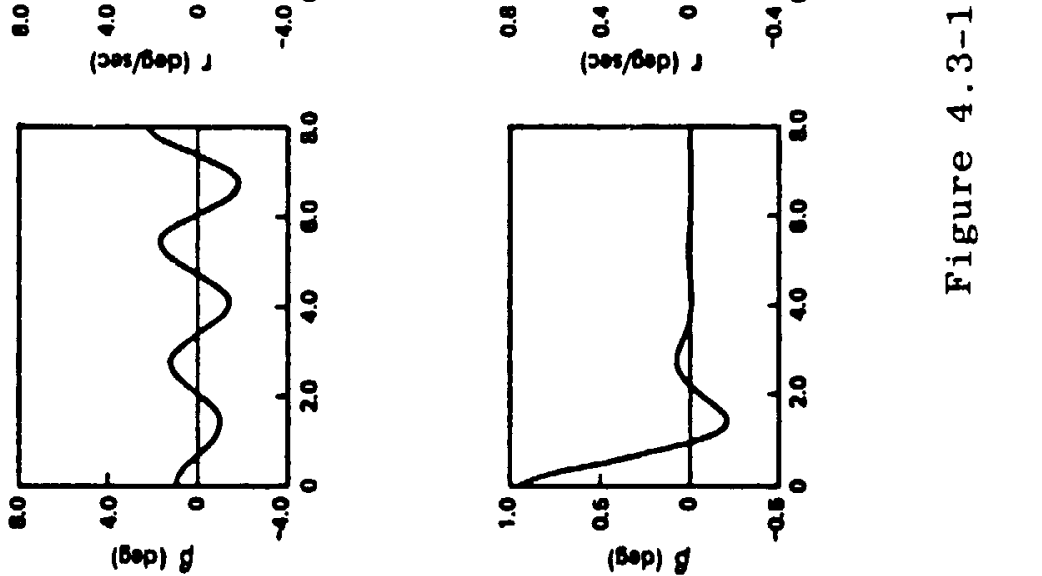
grows at a faster rate, and the closed-loop oscillation takes two cycles to disappear (in keeping with the reduced damping ratio of the Dutch roll mode).

\subsubsection{Lateral-Directional Sweep}

Nominal values of sideslip angle and stability axis roll rate are varied in this section, and their effects on closed-10op eigenvalues, DPSAS control gains, and aircraft response are presented. The development of this section follows the previous section, although the results presented for asymmetric flight are somewhat different from those of the longitudinal sweep. The $Q$ and $R$ matrices are the same as hefore, and linear-optimal regulators are designed at 18 points. Sideslip angles of 0,5 , and $10 \mathrm{deg}$ are considered in combination with stability-axis roll rates of $0, \pm 13, \pm 26$, and $\pm 39 \mathrm{deg} / \mathrm{sec}$. (For a given sideslip angle, roll rates of opposite sign have different dynamic effects.) Angle of attack, velocity, and altitude are fixed at $15 \mathrm{deg}$, $9 c_{1} \mathrm{~m} / \mathrm{s}$, and $6100 \mathrm{~m}$, respectively.

Table 4.3-3 presents the natural frequencies, damping ratios, and time constants of the aircraft, with the linear-optimal regulator loops closed. The most striking result, in comparison with Table 4.3-1, is that the lateral-directional closed-loop roots evidence relatively little variation with maneuver condition. There are no roll-spiral or phugoid degeneracies, and all parameters stay within 40 percent of their mean values. Short period, Dutch roll, and phugoid natural frequencies decrease with increasing $\beta_{0}$ magnitude and increase with increasing $p_{W_{0}}$ magnitude. Roll time constant and damping of the short period and phugoid modes are largely independent of $B_{0}$ magnitude but decrease with $p_{W O}$ magnitude. Dutch roll 
TABLE $4.3-3$

CLOSED-LOOP STABILITY IN THE LATERAL-DIRECTIONAL SWEEP

\begin{tabular}{|c|c|c|c|c|c|c|c|c|c|}
\hline \multicolumn{2}{|c|}{$\begin{array}{l}\text { Naneuver } \\
\text { Cond1tioa }\end{array}$} & \multicolumn{2}{|c|}{ Short Perlod } & \multicolumn{2}{|c|}{ Dutch Roll } & \multirow{2}{*}{$\frac{\text { Roll }}{\text { tec }}$} & \multirow{2}{*}{$\frac{\text { Sp1ral }}{\text { tec }}$} & \multicolumn{2}{|c|}{ Phugold } \\
\hline $\begin{array}{l}\text { Bo' } \\
\text { deg }\end{array}$ & $\begin{array}{c}P_{\text {wo' }} \\
\text { dec/sec }\end{array}$ & $\begin{array}{c}\omega_{\mathrm{n}} \\
\mathrm{rad} / \mathrm{sec}\end{array}$ & 6. & $\begin{array}{c}\omega_{n} \cdot \\
\mathrm{rad} / \mathrm{sec}\end{array}$ & 6. & & & $\begin{array}{c}\omega_{a} \cdot \\
\mathrm{rad} / \mathrm{gec}\end{array}$ & 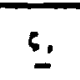 \\
\hline \multirow[t]{4}{*}{0} & 39 & 2.95 & 0.56 & 2.52 & 0.91 & 0.32 & 1.13 & 0.34 & 0.77 \\
\hline & 26 & 2.79 & 0.60 & 2.35 & 0.84 & 0.40 & 1.09 & 0.26 & 0.80 \\
\hline & 13 & 2.04 & 0.66 & 2.40 & 0.75 & 0.37 & 1.07 & 0.19 & 0.89 \\
\hline & 0 & 2.67 & 0.72 & 2.35 & 0.69 & 0.36 & 1.07 & 0.16 & 0.99 \\
\hline \multirow[t]{7}{*}{5} & -39 & 2.95 & 0.56 & 2.48 & 0.93 & 0.52 & 1.07 & 0.35 & 0.77 \\
\hline & -26 & 2.78 & 0.61 & 2.28 & 0.85 & 0.38 & 1.06 & 0.27 & 0.78 \\
\hline & -13 & 2.65 & 0.66 & 2.35 & 0.76 & 0.38 & 2.06 & 0.20 & 0.87 \\
\hline & 0 & 2. . 4 & 0.72 & 2.35 & 0.69 & 0.35 & 1.07 & 0.16 & 0.99 \\
\hline & 13 & 2.59 & 0.66 & 2.45 & 0.75 & 0.36 & 1.09 & 0.19 & 0.91 \\
\hline & 26 & 2.76 & 0.60 & $>10$ & 0.83 & 0.38 & 1.14 & 0.26 & 0.81 \\
\hline & 39 & 2.92 & 0.56 & 2.51 & G. 90 & 0.46 & 1.22 & 0.34 & 0.78 \\
\hline \multirow[t]{7}{*}{10} & -39 & 2.87 & 0.56 & 1.04 & 0.92 & 0.32 & 1.06 & 0.35 & 0.76 \\
\hline & -26 & 2.71 & 0.62 & 2.06 & 0.83 & 0.31 & 1.05 & 0.28 & 0.78 \\
\hline & -13 & 2.60 & 0.69 & 2.16 & 0.73 & 0.30 & 1.06 & 0.20 & 0.86 \\
\hline & 0 & 2.63 & 0.73 & 2.16 & 0.67 & 0.30 & 1.08 & 0.16 & 0.89 \\
\hline & 13 & 2.50 & 0.71 & 2.31 & 0.70 & 0.31 & 1.11 & 0.19 & 0.92 \\
\hline & 26 & 2.65 & 0.61 & 2.25 & 0.81 & 0.32 & 1.18 & 0.27 & 0.82 \\
\hline & 39 & 2.82 & 0.56 & 2.21 & 0.89 & 0.34 & 1.30 & 0.35 & 0.19 \\
\hline
\end{tabular}

damping increases with $\mathrm{p}_{W_{0}}$ magnitude and is 1 ittle affecter by $B_{0}$. The spiral mode time constant increases with $p_{W_{O}}$ magnitude, although its minimum value occurs at more negative $\mathrm{p}_{W_{0}}$ as $B_{0}$ increases.

Examples of the DPSAS gain variations with sideslip angle and roll rate are plotted in Fig. 4.3-2 and 4.3-3. The most apparent trend is that primary gains, i.e., those which would be non-zero in symmetric flight, charge very little with $\beta_{0}$ and $\mathrm{p}_{W_{0}}$ while crossfeed gains rave sub- 

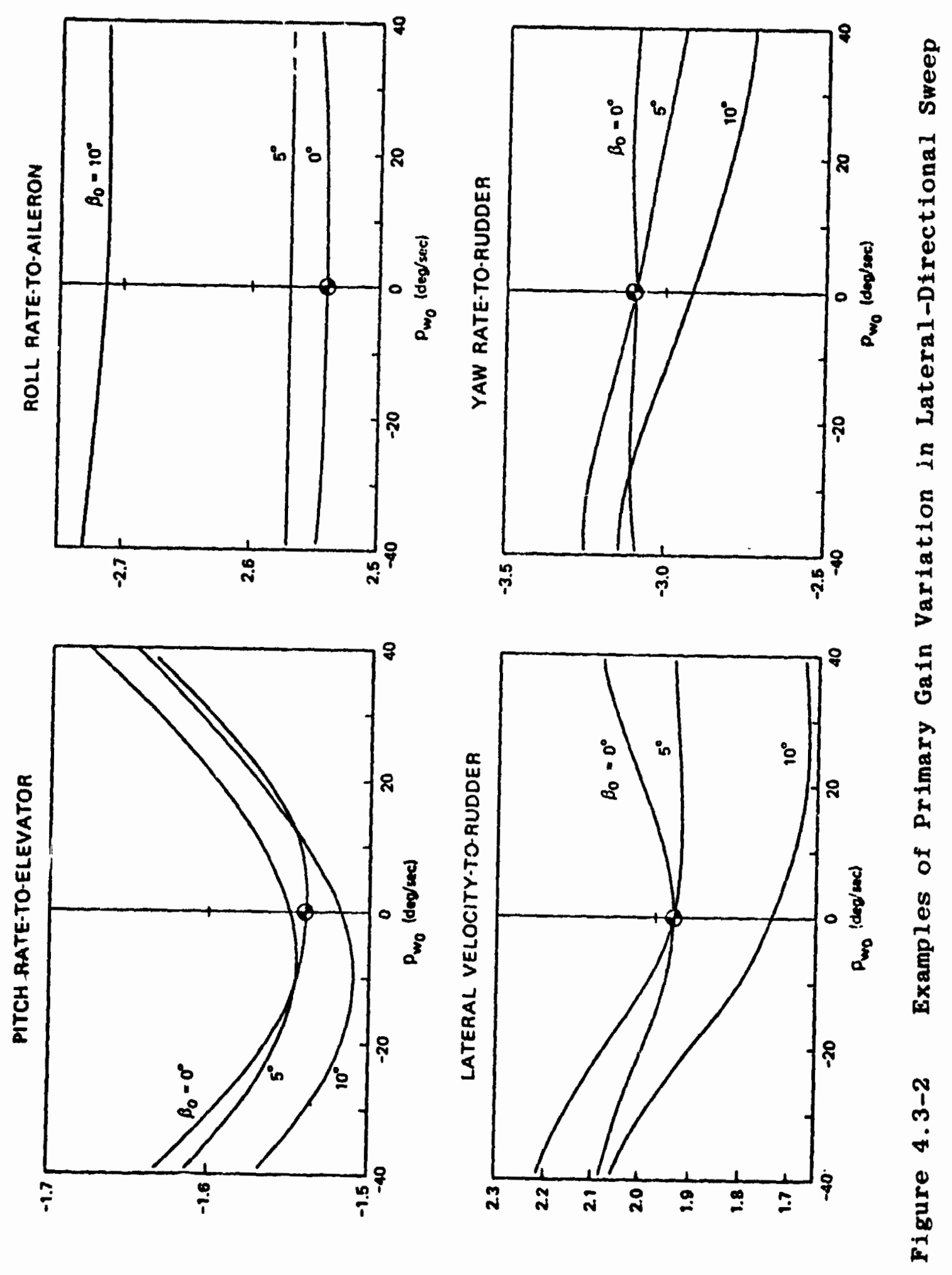

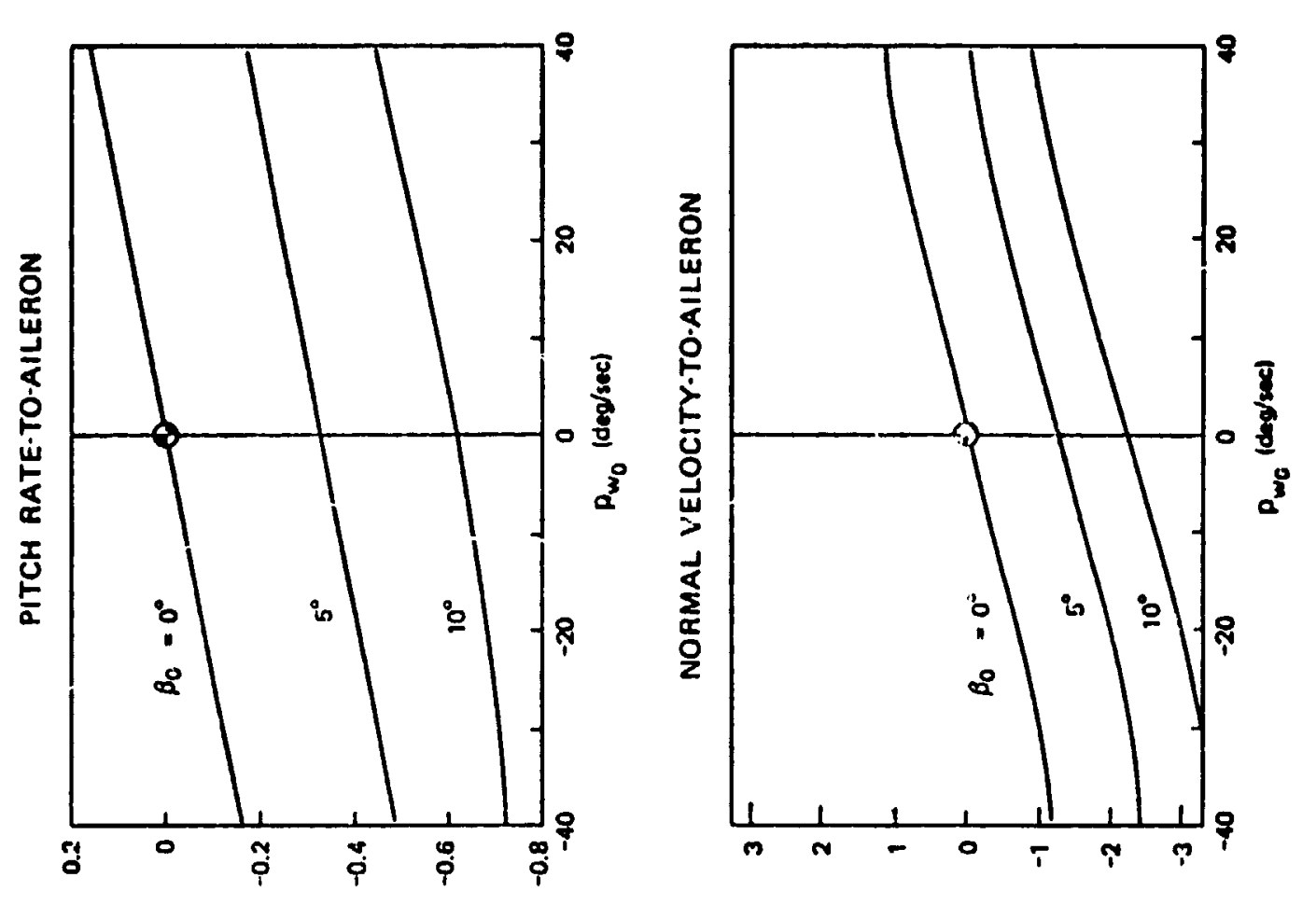

0.
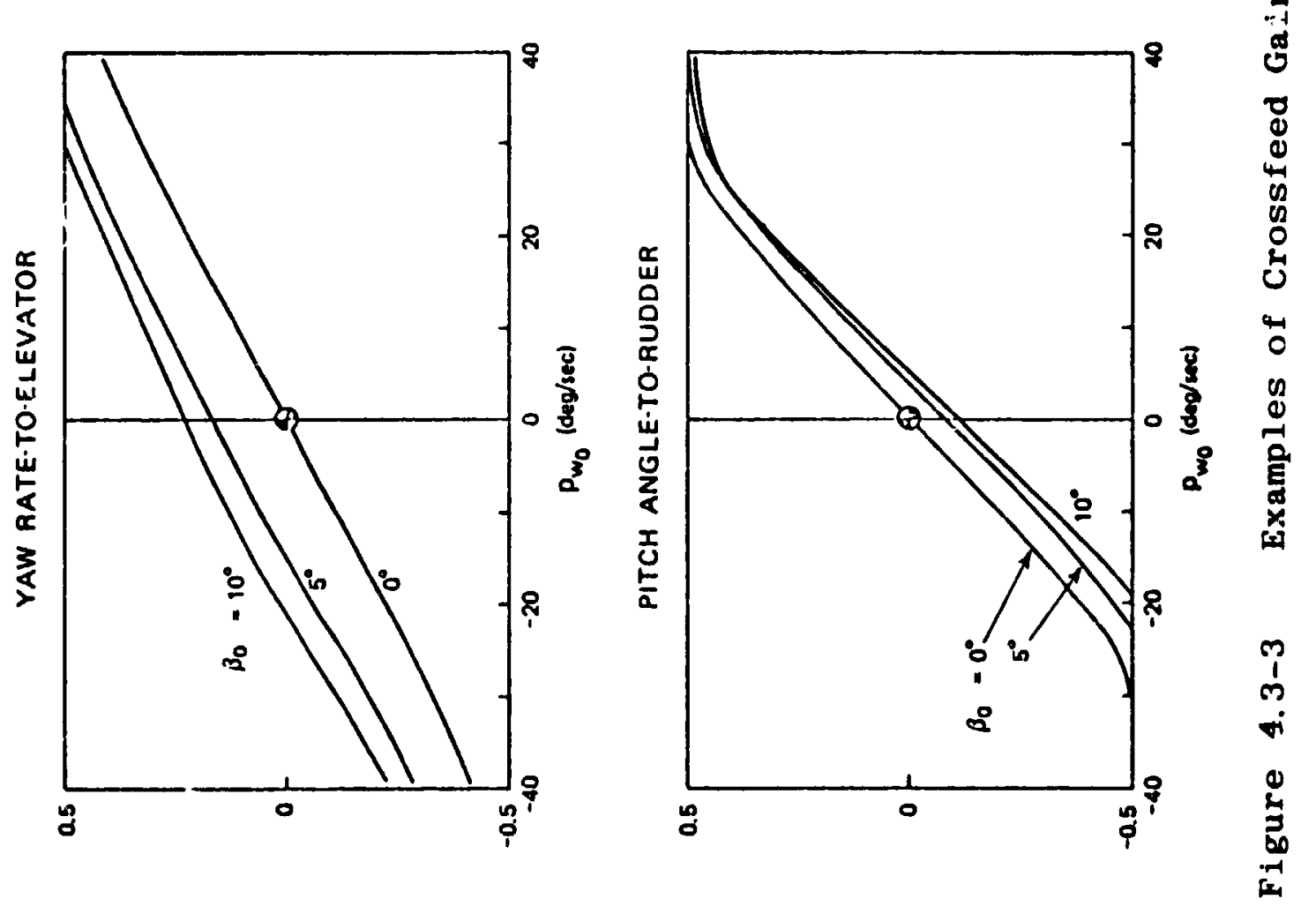
stantial variation with maneuver condition. The standard deviation of each gain, computed over the 18 literaldirectional sweep conditions, is an indication of its variation from a constant value. The average standard deviation for the primary gains is 16 percent, and for the crossfeed gains it is 422 percent. As discussed in Section 4.6, this is a first indication of gain-scheduling requirements, suggesting that many primary gains are nearly constant and that most secondary gains must be scheduled (unless they are negligible).

Gain variations are seen to depend on whether the vehicle is sideslipped "into" or "out of" the roll. (The vehicle is sideslipped into the roll when $B_{0}$ and $p_{W_{0}}$ have opposite sign, e.g., when the nose is left and the left wing is moving down; it is sideslipped out of the roll when the signs are equal.) Figures 4.3-2 and 4.3-3 illustrate gain variations for positive $B_{0}$ only; for negative $B_{0}$, the variations with $p_{W_{0}}$ are changed. The graphs of primary gains for negative $B_{0}$ are mirror images of those for positive $B_{0}$ (Fig. 4.3-2). The graphs of crossfeed gains for negative $B_{0}$ shift up or down, in opposition to the $\beta_{0}$ trend shown in Fig. 4.2-3 Primary gains can be monotonic or convex functions of $\mathrm{p}_{\mathrm{W}_{0}}$; crossfeed gains are monotonic in $p_{W D}$ and always pass through zero when both $B_{0}$ and $p_{W_{O}}$ are zero (Gains for symmetris flight are inidcated by in Fig. 4.3-3).

The crossfeed gains are shown to be non-trivial for even moderate values of $B_{O}$ and $p_{W_{0}}$, and those shown in Fig. 4.3-3 can be interpreted as nonlinear control elements.

Note that each gain could be approximated by a function of the form

$$
\operatorname{Gai} 1=c_{1} B_{0}+c_{2} p_{w_{0}}
$$




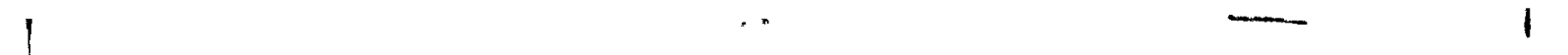

where $c_{1}$ and $c_{2}$ are appropriate constants. Then the control signals represented by these four graphs would be

$$
\begin{aligned}
& \Delta \delta_{h}=c_{1} \beta{ }_{0} \Delta r+c_{2} p_{w_{0}} \Delta r \\
& \Delta \delta_{a}=c_{3} \beta{ }_{0} \Delta q+c_{4} p_{W_{0}} \Delta q \\
& \Delta \delta_{r}=c_{5}{ }^{\beta} 0^{\Delta \theta}+c_{6} p_{W_{0}} \Delta \theta \\
& \Delta \delta_{a}=c_{7}{ }_{0} \Delta w+c_{8} p_{W_{0}} \Delta w
\end{aligned}
$$

where the constants are derived hy rerression analysis (Section 4.6). The $\mathrm{p}_{W_{0}} \Delta \mathrm{w}$ and $\mathrm{p}_{W_{0}} \Delta \theta$ terms can be recognized as analogous to so-called "pseudo- $\dot{B}$ " or "pa" crossfeeds, which have been incorporated in the SAS of modern high-performance aircraft. (An aaditional "pa"-tyne primary gain is indicated in Table 4.3-2. The roll rate-to-rudder gain could be approximated by $c \alpha_{0}$; therefore, the associated rudder command would be $\left.c \alpha_{0} \Delta \mathrm{p}.\right)$ Nonlinearities in the curves of Fig. 4.3-3 suggest that higher-order fits than Eq. (4.3-1) to (4.3-5) are required if design performance is to be obtained over a wide range of $B_{0}$ and $\mathrm{p}_{W_{0}}$.

Examples of open- and closed-loop response at two asymmetric flight conditions are shown in the next two figures. Figures $4.3-4$ and 4 .3-5 show that roll rate introduces substantial longitudinal response to a directional input and that the addition of sideslip angle leads to qualitative changes in response shapes. Roll rate alone introduces regular oscillations in the aircraft's open-loop response (Fig. 4.3-4). The DPSAS damps the oscillation within $1 \frac{1}{2}$ cycles, although excitation of the phigoid mode leads to a slow decay in $\Delta \alpha$ (The effective time constant $\left(-\zeta \omega_{n}\right)$ of the phugoid is 6 sec at this flight condition). 

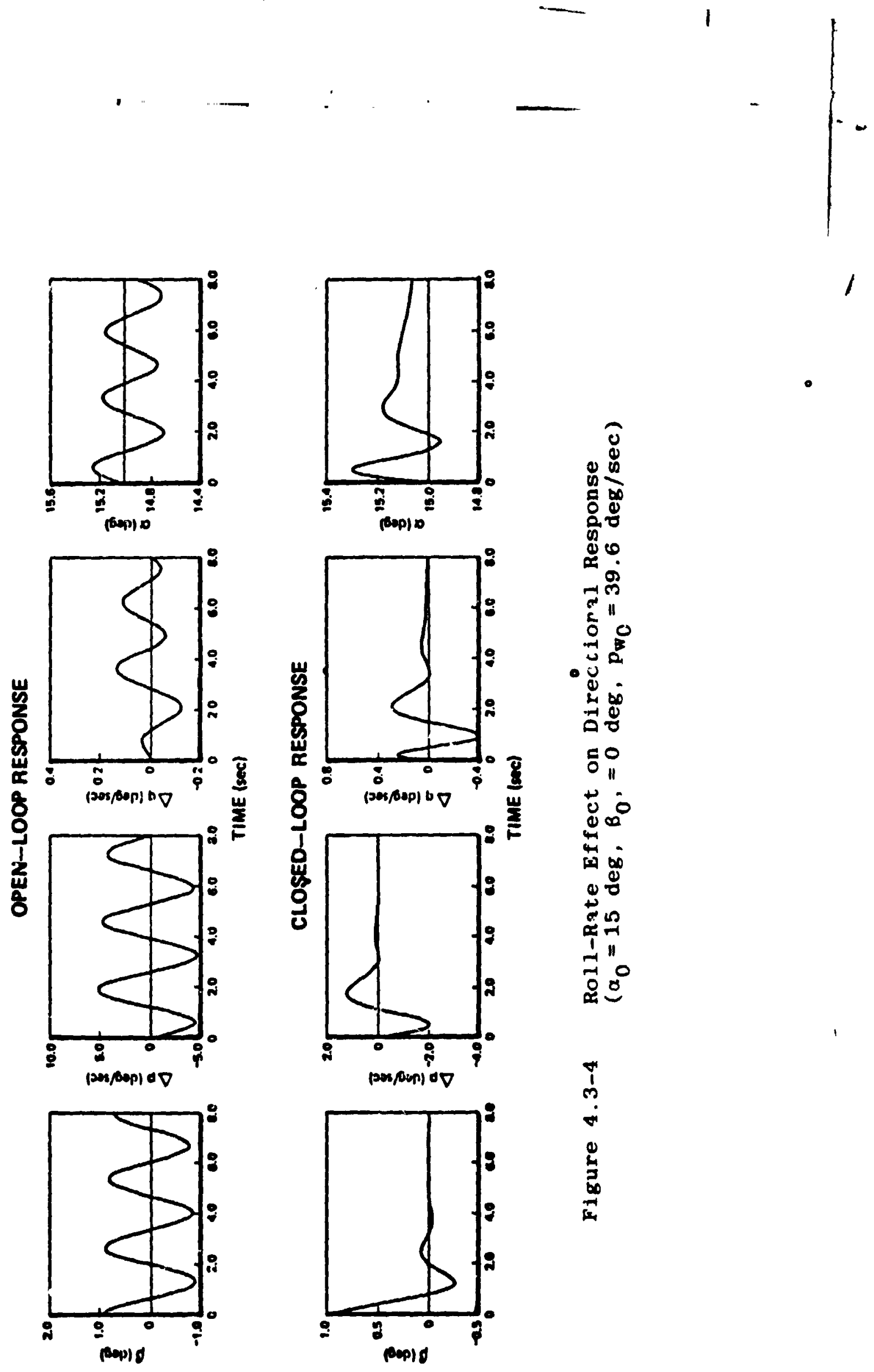

1

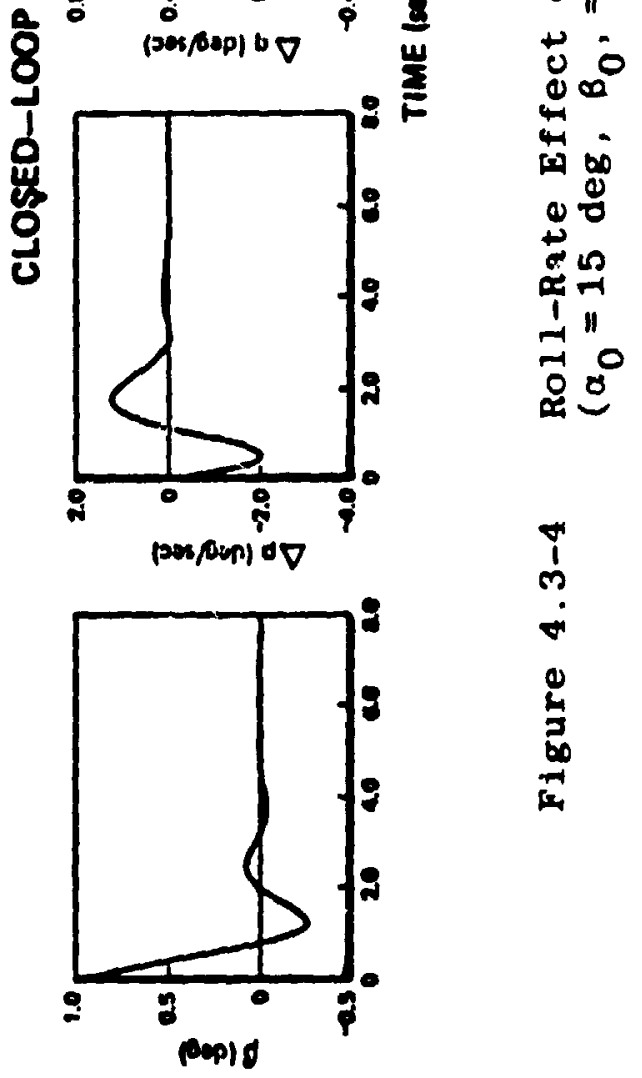



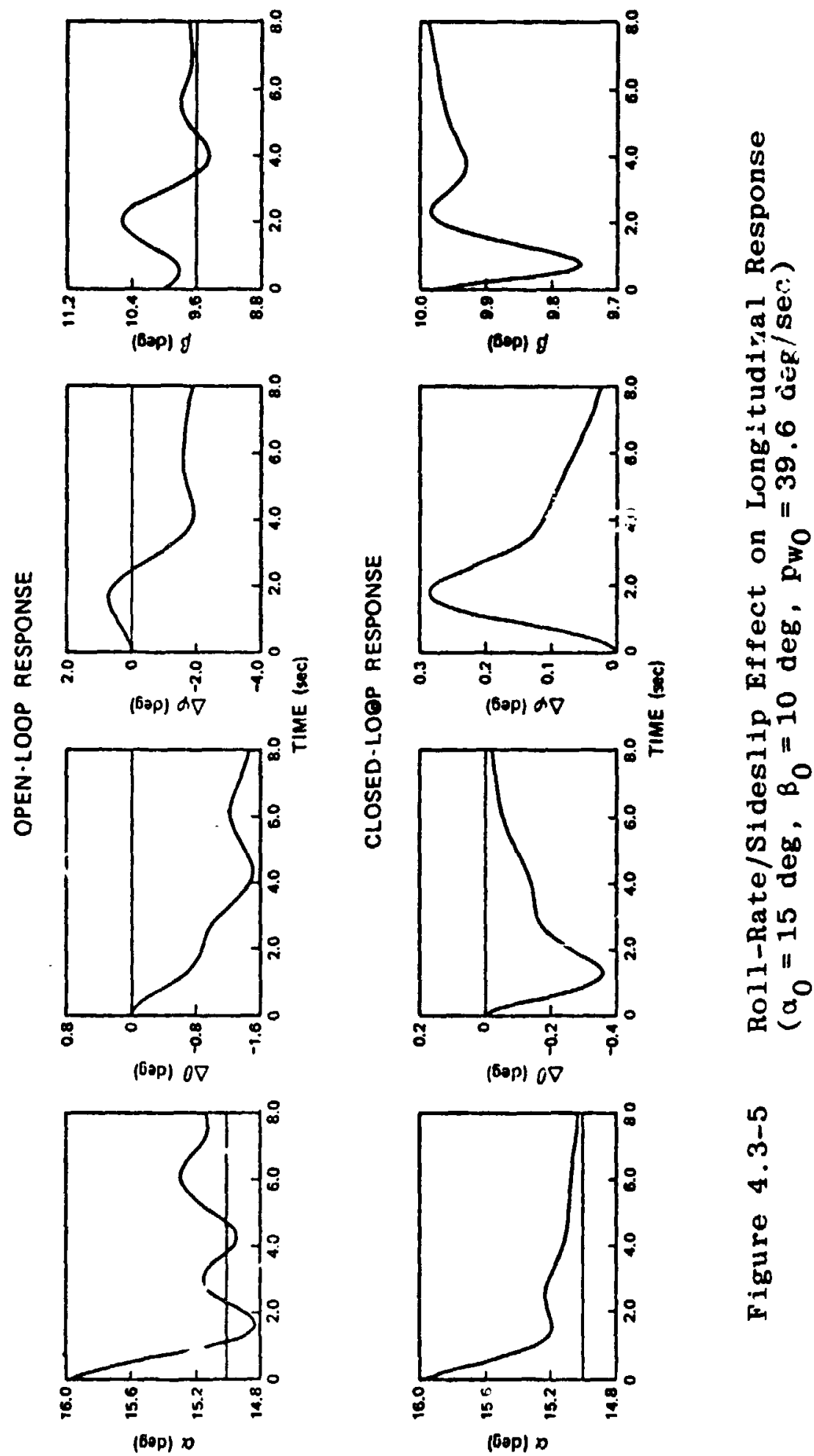
When the aircraft has developed a large mean sideslip angle as well as roll rate, the open-loop perturbation motions tend to meander, as several modes are involved in each motion (Fig. 4.3-5). For example, the initial $\Delta \alpha$ appears to be damping out, but after $5 \mathrm{sec}$, it begins to wander. Pitch and roll angle develop offsets which are continuing to increase at the end of the time period shown. The DPSAS restricts the maximum initial excursions of $\Delta \theta$, $\Delta \phi$, and $\Delta B$ to less than half their open-loop values and eliminates the meandering characteristic.

Plotting $B$ rather than $\Delta B$ in Fig. 4.3-5 is a reminder that the DPSAS provides stab. lity about a reference flight condition, in this case, 10-deg sideslip angle and $-39.6-\mathrm{deg} / \mathrm{sec}$ roll rate. With the assumption that these values are commanded by the pilot, it can be seen that the DPSAS does not limit aircraft maneuverability -- in fact, it expands the flight envelope by stabilizing the aircraft in conditions which could not be controlled by the unaided pilot. Although non-zero $B_{O}$ is not normally desired in maneuvering current high-performance aircraft, future aircraft, particularly those with direct side force control, could use this capability to tactical advantage.

This section has presented linear-optimal DPSAS designs for the reference aircraft and for a variety of maneuvering conditions. The next section of this chapter deronstrates how control gains can be adapted to flight condition. 


\subsection{CONTROL-LAW ADAPTATION FOR VARYING FLIGHT CONDITIONS}

This secticn presents results for a procedure which adapts the control gains of a high-performance aircraft to varyiug flight conditions, including gain correlations for the reference aircraft model. The gains are scheduled by finding functional relationships between aircraft flight variables and the control gains at the corresponding flight conditions.

Previous methods for scheduling control gains have been successful and indicate that gain scheduling is a sound approach. The methodology typically is based on single input/single output concepts, e.g., maintaining constant loop gain. These previous methods, however, provide inadequate insight for scheduling a multivariable system.

The method is a logical extension of previous work to multivariable systems. It involves three steps, and it places minimum reliance on past experience and intuition. The three steps are:

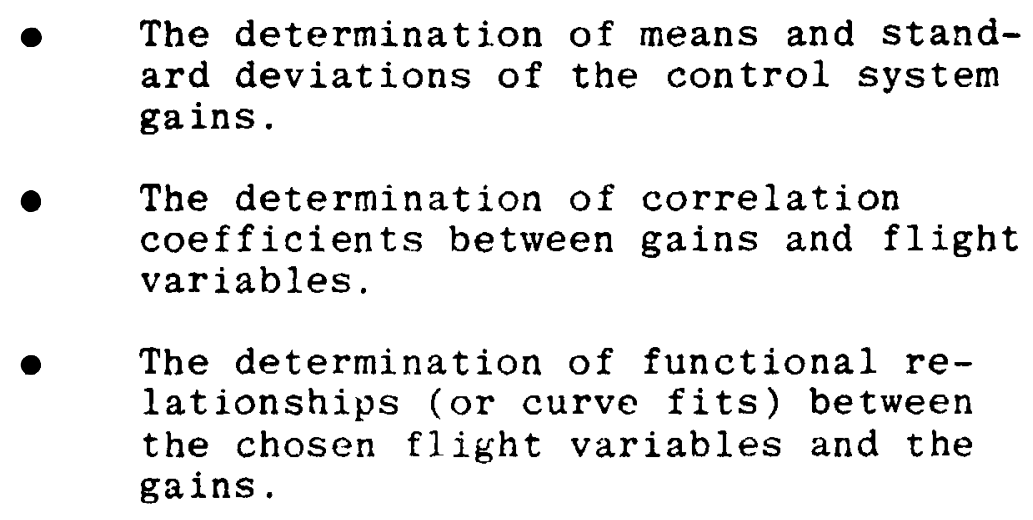

This new gain scheduling procedure, discussed in Section A.4.5, 
is simple to use, the results are easy to implement on a digital computer, and the procedure can have broad application.

The longitudinal and lateral-directional sweep control gains discussed in Section 4.3 have been correlated with a number of flight variables. In order to identify the individual effects of longitudinal and lateral-directional mean motions, the correlations for each sweep are done separately. In a flight system, the gains should be correlated jcintly, and additional factors - such as weight, altitude, and velocity -- must be considered.

For each sweep, a list of candidate independent variables is established, and various functions of their variables are correlated with the 32 gains associated with each flisit condition. Functions considered insluded polynomials of order one and two,

$$
\begin{aligned}
& \text { Gain }=b_{0}+b_{1} m \\
& \text { Gain }=b_{0}+b_{1} m+b_{2} m^{2}
\end{aligned}
$$

and linear regressions in two variables,

$$
\text { Gain }=b_{0}+b_{1} m_{1}+b_{2} m_{2}
$$

Given a flight variable, $y$, indepencient variables, $m$, of the form $y, y^{2}, 1 / y, 1 / y^{2}$, and $y|y|$ are considered in the polynomial regressions. Equation $(4.4-3)$ is vised with $m_{1}=y_{1}$ and $m_{2}=y_{2}$. The objective of the computations is to find the functional approximation to each gain which has the greatest correlation with the linear-optimal gain at all conditions in the particular sweep. In many cases, alternate functions have similar correlation coefficients, so more than one schedule could be considered in implementation. 
The correlation between gains also is of interest, as it suggests which gains can be scheduled as functions of other gains, and it helps to identify control interconnects.

- This correlation can be computed, using Eq. (A.4-37), by defining the first gain as $k$ and the second as $\hat{k}$. The following results indicate that an aileron-rudder interconnect could be considered for the stability augmentation system of the reference alrcraft.

\subsubsection{Longitudinal Sweep}

The procedure followed in establishing gain-scheduling requirements is to compute means and standard deviations (as percentages of the means) of tue gains, to correlate gains with flight variable functions, and to correlate gains with other gains. Sixteen crossfeed gains are identically zero, leaving sixteen gains for scheduling.

Table 4.4-1 summarizes the findings for DPSAS gains in the longitudinal sweep, presenting the mean and standard deviation of each gain. The independent variables which provide the best gain schedule are listed, along with the correlation between the actual and scheduled gain values. Also listed is the gain which exhibits the highest crosscorrelation, (calculated by applying Eq. (A.4-37) to all pairs of gains) and the value of that cross-correlation. For example, the gain $\Delta \delta_{T} / \Delta \theta$ exhibits a mean of -0.016 and a standard deviation of $32 \%$ over the chosen set of longitudinal flight conditions. A gain schedule using normal load factor $\left(n_{z_{0}}\right)$ and pitch rate $\left(q_{0}\right)$ produces a scheduled gain whose correlation factor with the actual gain is 0.89 . Finally, $\Delta \delta_{\mathrm{T}} / \Delta \theta$ exhibits strongest cross-correlation with $\Delta \delta_{\mathrm{T}} / \mathrm{u}$, and the correlation factor is 0.98 . The flight variables considered as possible scheduling variables are 
TABLE $4.4-1$

GAIN CORRELATIONS FOR THE LONGITUDINAL SWEEP

- Longitudinal Gains

\begin{tabular}{|l|r|r|r|r|r|r|r|r|}
\hline \multicolumn{1}{|c|}{ Gain } & $\Delta \delta_{\mathrm{T}} / \Delta \theta$ & $\Delta \delta_{\mathrm{T}} / \Delta \mathrm{u}$ & $\Delta \delta_{\mathrm{T}} / \Delta \mathrm{q}$ & $\Delta \delta_{\mathrm{T}} / \Delta \mathrm{W}$ & $\Delta \delta_{\mathrm{h}} / \Delta \theta$ & $\Delta \delta_{\mathrm{h}} / \delta_{\mathrm{u}}$ & $\Delta \delta_{\mathrm{h}} / \Delta \mathrm{q}$ & $\Delta \delta_{\mathrm{h}} / \Delta \mathrm{w}$ \\
\hline $\begin{array}{l}\text { Mean of Gain } \\
\text { Standard Deviation } \\
\text { of Gain, \% of Mean } \\
\begin{array}{l}\text { Best Scheduling } \\
\text { Variables }\end{array}\end{array}$ & -0.016 & 0.062 & -0.006 & 0.013 & -0.763 & 1.640 & -1.621 & -0.928 \\
$\begin{array}{l}\text { Scheduled/Actual } \\
\text { Gain Correlation }\end{array}$ & 0.89 & 0.93 & 0.95 & 0.95 & 0.85 & 0.94 & 0.88 & 0.89 \\
$\begin{array}{l}\text { Gain of Highest } \\
\text { Cross Correlation } \\
\text { Gain Cross } \\
\text { Correlatiol. }\end{array}$ & $\Delta \delta_{\mathrm{T}} / \Delta \mathrm{u}$ & $\Delta \delta_{\mathrm{T}} / \Delta \theta$ & $\Delta \delta_{\mathrm{h}} / \Delta \mathrm{u}$ & $\Delta \delta_{\mathrm{h}} / \Delta \mathrm{q}$ & $\Delta \delta_{\mathrm{h}} / \Delta \mathrm{q}$ & $\Delta \delta_{\mathrm{T}} / \Delta \mathrm{q}$ & $\Delta \delta_{\mathrm{a}} / \Delta \mathrm{r}$ & $\Delta \delta_{\mathrm{a}} / \Delta \phi$ \\
\hline
\end{tabular}

Lateral Gains

\begin{tabular}{|c|c|c|c|c|c|c|c|c|}
\hline Gain & $\Delta \delta_{a} / \Delta v$ & $\Delta \Delta \delta_{\mathrm{a}} / \Delta \mathrm{r}$ & $\Delta \delta_{\mathrm{a}} / \Delta \mathrm{p}$ & $\Delta \delta_{a} / \Delta \phi$ & $\Delta \delta_{\mathrm{r}} / \Delta \mathrm{v}$ & $\Delta \delta_{\mathrm{r}} / \Delta \mathrm{v}$ & $\Delta \delta_{r} / \Delta p$ & $\Delta \delta_{r} / \Delta \phi$ \\
\hline Mean of Gain & -0.446 & 0.076 & 2.054 & 1.764 & 2.480 & -3.567 & 0.404 & -0.206 \\
\hline $\begin{array}{l}\text { Standard Deviation } \\
\text { of Gain, } \% \text { of Mean }\end{array}$ & 251 & 1713 & 50 & 40 & 50 & 32 & 60 & 111 \\
\hline $\begin{array}{l}\text { Best Scheduling } \\
\text { Variables }\end{array}$ & $\cos \alpha$ & $\cos a$ & $\cos \alpha$ & $\cos \alpha$ & $\cos \alpha$ & $\cos \alpha$ & $\mathrm{n}_{\mathbf{z}}$ & $\mathrm{n}_{\mathrm{z}}, \mathrm{q}$ \\
\hline $\begin{array}{l}\text { Scheduled/Actual } \\
\text { Gain Correlation }\end{array}$ & 0.93 & 0.83 & 0.82 & 0.90 & 0.93 & 0.91 & 0.94 & 0.98 \\
\hline $\begin{array}{l}\text { Gain of llighest } \\
\text { Cross Correlation }\end{array}$ & $\Delta \delta_{\alpha} / \Delta \phi$ & $\Delta \delta_{2} / \Delta \phi$ & $\Delta \delta_{r} / \Delta r$ & $\Delta \delta_{\mathbf{r}} / \Delta \mathbf{r}$ & $\Delta \delta_{r} / \Delta r$ & $\Delta \delta_{r} / \Delta v$ & $\Delta \delta_{h} / \Delta w$ & $\Delta \delta_{\mathrm{T}} / \Delta u$ \\
\hline $\begin{array}{l}\text { Gain Cross } \\
\text { Correiation }\end{array}$ & 0.94 & 0.98 & 0.98 & 0.99 & 1.00 & 1.00 & 0.89 & 0.90 \\
\hline
\end{tabular}

*AIl Independent variables evaluated at nominal flight condition, " 0 " subscript omitted. 

with little performance degradation, and a moderate possibility for combining $\Delta p$ and $\Delta \phi$ feedbacks as well.

\subsubsection{Lateral-Directional Sweep}

Correlation results for the lateral-directional sweep are shown in Table 4.4-2 which indicates that no gain means are identically zero, although several appear negligible. The flight variables considered for scheduling are sideslip angle $\left(B_{0}\right)$ and stability-axis roll rate $\left(\mathrm{p}_{0}\right)$, which are chosen to illustrate the DPSAS gain scheduling method. In an actual application, additional independent variables could be included in the search.

Unlike the longitudinal sweep, it appears that 13 gain:s cculd be crnsidered constant, with standard deviations of less than 8 percent of the mean value. Five gains are inadequate?y scheduled by the chosen independent variables and functions, as their correlations are below 0.75. Two of these are the rudder gains shown in Fig. 4.3-2, which can be seer to be more complex than the polynomials and linear combination considered here. Higher-order curves would fit these gains, although they are candidates for the constant-value approximation because their standard deviations are low.

Seventeen gains are most closely correlated with $p_{W_{0}}$ and are fitted best by second-order polynomials in $\mathrm{pW}_{0}$. The eleven gains which are most correlated with $B_{0}$ are fitted almost as well by second-order polynomials in $\beta_{0}, B_{0}^{2}, 1 / \beta_{0}$, $1 / B_{0}^{2}$, or $B_{0}\left|B_{0}\right|$. Three of the four linear $B_{0}-p_{W_{0}}$ fits are adequate, with $\Delta \delta_{\mathrm{r}} / \mathrm{\Delta r}$ requiring an improved fit (along with $\Delta \dot{\delta}_{\mathrm{a}} / \Delta \mathrm{w}, \Delta \delta_{\mathrm{a}} / \Delta \mathrm{v}, \Delta \delta_{\mathrm{a}} / \Delta \mathrm{r}$, and $\left.\Delta \delta_{\mathrm{r}} / \Delta \mathrm{v}\right)$. Most pairs have strong correlation with at least one other gain. The correlations associated with SAS aileron-ruader interconnect are $0.95(\Delta v)$, 


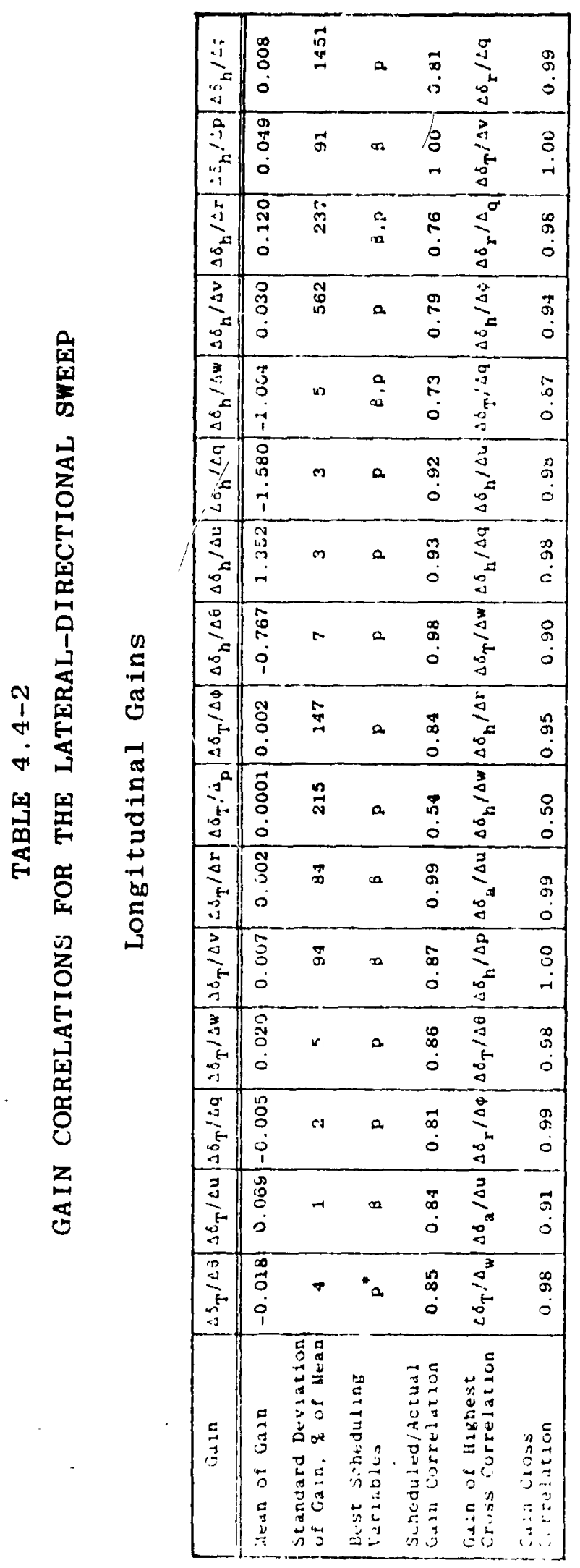

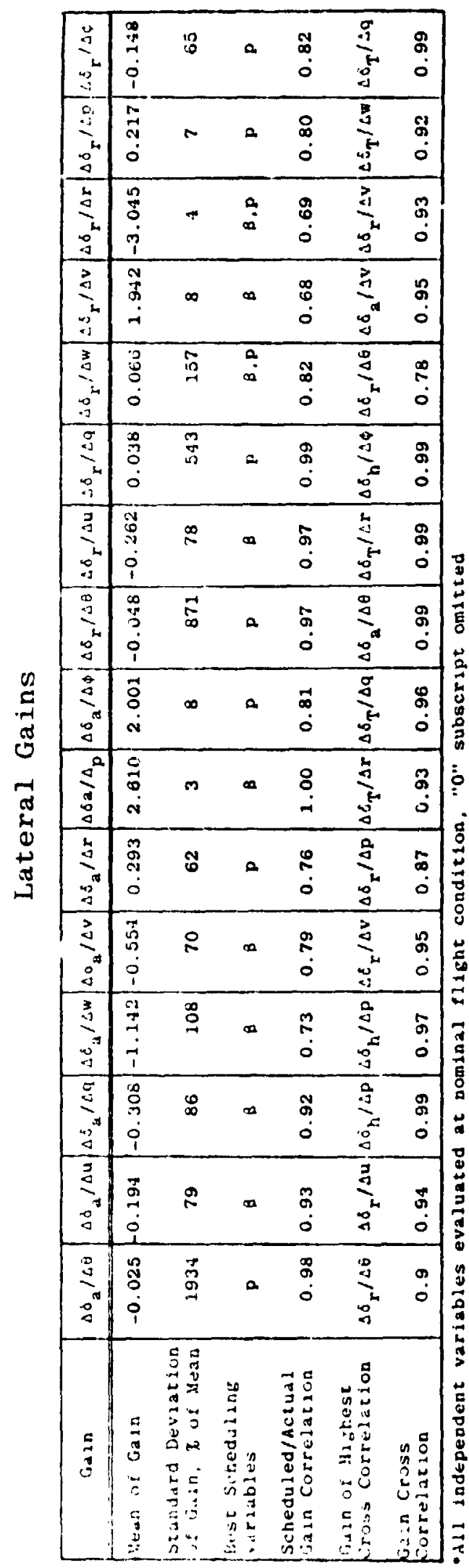


$0.50(\Delta r), 0.13(\Delta p)$, and $0.04(\Delta \phi)$, indicating that either rudder or aileron would require additional $\Delta r$ and $\Delta p$ feedbacks in parallel with the interconnected control path.

\subsubsection{Additional Considerations}

The longitudinal and lateral-directional sweeps were conducted to illustrate the separate effects of $\alpha_{0}, a_{0}, \beta_{0}$, and $\mathrm{p}_{W_{0}}$ on DPSAS gains. Furthermore, a limited set of independent variables and scheduling functions were examined. At a minimum, these sweeps should be combined in a single correlation procedure to obtain a single multi-variable schedule for each gain. Altitude, velocity, and weight effects should be added, principally through indicated airspeed, Mach number, and the ratio of weight-to-dynamic pressure. Permutations of the independent variables, e.g., body-axis rather than stability-axis rates, may provide better correlation or may be easier to implement in a particular system.

The present results suggest that primary gains schedule largely on longitudinal variables and that crossfeed gains schedule primarily on lateral-directional var1ables. This observation derives from the fact that most primary DPSAS gains are nearly constant as $\beta_{0}$ and $p_{\text {po }}$ change, while crossfeed gains are zero in symmetric flight. Any approximations made in gain scheduling must be validated by direct simulation, as this is tantamount to changing the gains from their linear-optimal values, thus altering closed-loop response.

An entirely separate issue is the on-board determination (either through measurement or estimation) of the independent variables to be used for gain scheduling -- 
particularly $\alpha_{0}$ and $\beta_{0}$, which are notably difficult to measure. Two potential problems are inaccurate steadystïie measurement, which leads to inaccurate calculation of gains, and superposition of perturbations on the mean values, which could cause longitudinal motions to drive lateraldirectional motjons (and vice versa) through oscillatory gain changes. The solution to both problems, should they occur, is found through state estimation, which allows all available measurements to be blended in a unified estimate of nominal and perturration motion variables (Ref. 63). As an example, measurements of $\alpha, q, n_{z}$, airspeed, and $\delta_{h}$ could be used to estimate $\alpha_{0}, q_{0}, \Delta \alpha$, and $\Delta q$. If the DPSAS is incorporated in a full command augmentation system, pilot commands could be direct indicators of the desired (or nominal) state; therefore, they could be used for gain scheduling (Ref. 58). This is a tcpic for further study.

\subsection{CHAPTER SUMMARY}

This chapter has presented design principles for stability augmentation systems (DPSAS) which prevent departure from controlled flight. Linear-optimal control theory has been used to develop control structures for departure prevention, and the effects of maneuvering condition on optimal feedback and crossfeed gains have been explored. Examples of aircraft response to longitudinal, lateral, and directional initial conditions illustrate the well-controlled behavior which the DPSAS provides, and closed-1oop eigenvalues show that variation in alrcraft dynamic characteristics is minimized for a wide range of maneuvering conditions.

In many respects, stabilizing the reference alrcraft in a pullup maneuver is a more challenging task than accounting 
for the coupling which results from sideslip and roll rate; however, lateral-directional maneuvering results in significant linear-optimal gains which improve aircraft response. In combination with gain-scheduling functions which depend on mean values of angles and angular rates, the DPSAS control algorithms are seen to produce nonlinear crossfeeds which are analogous to control structures being employed in modern high-performance aircraft.

Linear-optimal control theory solves many aircraft control problems which have been difficult to overcome with past design techniques. It is easy to use, it guarantees system stability, and it accomnodates aircraft with limited control authority. 
This report has illustratec how linear systems analysis can be used to characterize the stability of aircraft during maneuvering flight. It also presents a design procedure for stability augmentation systems which prevent departure from controlled flight. The key to linearizing the dynamics of the aircraft is that an accelerated flight condition can be used as a reference path. A linear model can provide a good description of the aircraft's perturbation response (to initial conditions, control inputs, and disturbances) even when the aircraft has large aerodynamic angles and angular rates. Control sjstems designed for fully coupled linear models and adapied to changing flight conditions can provide protection against inadvertent departure from controlled flight.

\section{1 CONCLUSIONS}

A detailed examination of the dynamics of the reference aircraft has led to sentrallzations concerning aircraft stability and control. These include the following:

\footnotetext{
- The aircraft's stability (as shown by its eigenvalues) is most affected by changes in the nominal longitudinal variables $\left(v_{0}, a_{0}\right.$, and $\left.q_{0}\right)$, while the mode shapes (as described by the aircraft eigenvectors) are most affected by non-zero nominal values of the lateral variables ( $B_{U}$ and $\left.\mathrm{p}_{\mathrm{W}_{0}}\right)$. Asymmetric flight leads to iongitudinal-variable response in typically lateral-directional modes, and vice-versa.
} 
Nonminimum-phase zeros in the aircraft transfer functions occur frequently in asymmetric flight, and the transter function numerators are changed substantially by non-zero $q_{0}$.

- Extreme maneuvers are ofte. characterized by rapid changes in both mode srapes and speeds due to large values of $\alpha_{0}, p_{W_{0}}$, and $q_{0}$. Highly coupled, unstable natural modes can result.

- Elementary loop closures which are stabilizing in symmetic flight can lead to unstable system dynamics in asymmetric flight.

- The departure parameter, $C_{n_{B d y n}}$, has limited value in predicting aircraft departure. It provides no information regarding Dutch roll dampirg; hence, it does not predict departure due to negative damping (as is the case for the subject aircraft).

- Unforced departures occur when one of the fast modes (short period, Dutch roll, or roll mode) is unstable. These departures primarily take the form of a Dutch roll instability, with fast rolling-yawing motions or oscillatory divergence. The roll mode can become unstable at extreme angles, where it exhibits a fast rollyaw divergence.

- Forced departures occur as a result of pilot action. This can happen when degraded response to control inputs causes the pilot to $f 1 y$ the aircraft into a flight regime where unforced departures are likely or when pilot actions destabilize the aircraft directly.

- Guidelines for the design of DepartureFrovention Stability Augmentation Systems (DPSAS) have been presented. An adajtive-control design procedure, using the linear-optimal regulator for 
fixed-point design followed by gain scheduling, is shown to provide a nonlinear control structure containing crossfeeds as well as feedback gains. The resulting DPSAS has similarities to. the flight control systems of current high-performance aircrift. However, the new design is based on "quadratic synthesis" techniques, which provide a unified set of control gains for all axes from a single set of vector-matrix design equations.

- The linear-optimal DPSAS prevents departure not by limiting the maneuvering ability of the aircraft but by stabilizing the aircraft in all foreseeable maneuver conditions.

- The linear-optimal control law can be readily extended to a full Departure-Prevention Command Augmentation System (DPCAS) which accounts for control actuator rate 1 imits and allows essentially unlimited pilot control authority (within che physical limitations of the aircraft).

- The maneuverability envelope of the subject aircraft could be materially expanded through the incorporation of DPSAS/DPCAS concepts, as identified in this report.

\subsection{RECOMMENDATIONS}

The following recommendations are made as a result of this study:

- Departure prevention studies for highperformance aircraft should be extended to transonic and supersonic flight regimes.

- The high angle-of-attack/high angular rate problems of additional aircraft types, including trausports, helicopters, and general aviation aircraft, 
are amenable to coupled linear analy-

sis and bear investigation.

- Design requirements for a DPCAS should be investigated. Digital implementation and incorporation of active control concepts for improved maneuverability should be considered.

- It is recommenoed that improvements to the subject aircraft's maneuverability envelope due to DPSAS/DPCAS implementation be explored in ground-based piloted simulation and flight test.

Dynamic coupling is a significant factor in the maneuvers of high-performance aircraft, and a full understanding of its effects is an important facet of preventing departure from controlled flight. This report has shown how linear models of the aircraft's motions can be used to investigate the stability and control of maneuvering flight, and it has demonstrated the flexibility and ease with which linear-optimal control theory can be used to design departurepreventing control systems. 


in orientation with respect to the vehicle. Generally, the body $x$-axis extends forward out the vehicle's nose, the $y$-axis extends out the right wing, and the z-axis extends out the bottom of the vehicle. The $x-z$ plane is usually a plane of geometric symmetry, if the vehicle has one. There are a number of possible body-fixed reference frames, and the one fixed by the builder is simply referred to in this report as the body-axis system. For any nominal flight condition, body-fixed axes can be chosen so that the $x$-axis is aligned with the velocity vector, and the $z$-axis is in the bodyaxis $x-z$ plane. This set of body-fixed axes is referred to as the stability-axis system.

Since body axes are the only axes in which the vehicle rotational inertia matrix is constant, the rotational dynamics equations are usually (though not exclusively) expressed in this frame. The body frame also is the one in which the pilot, and all sensors and control surfaces are located; for this reason, the body frame is considered the basic frame of reference in this report. Figure A.2-1a illustrates the body frame orientation with respect to the inertial-axis system.

The velocity- and wind-axis systems have a common origin (the vehicle center of mass) and a common $x$-axis, which is oriented along the vehicle's inertial velocity vector. The velocity reference frame $y$-axis is parallel to the inertial $x-y$ plane. This results in simple relations between inertial and velocity axes, so that these axes are useful for navigation and point-mass trajectory calculations. Figure A.2-1b illustrates the relationship between inertial and velocity axes.

The wind reference frame's $z$-axis is located in the $x-z$ plane of the body frame; this reference frame is 


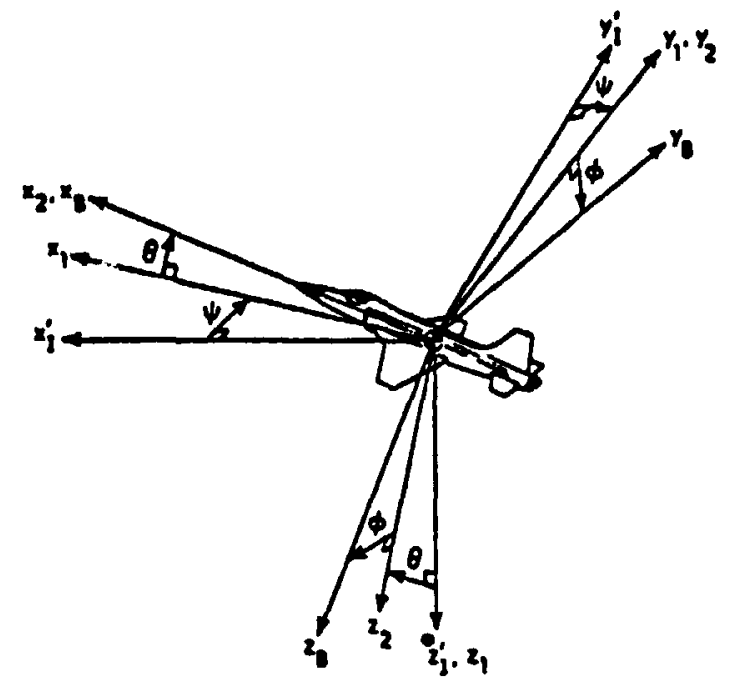

a) Inertial-Body Orientation

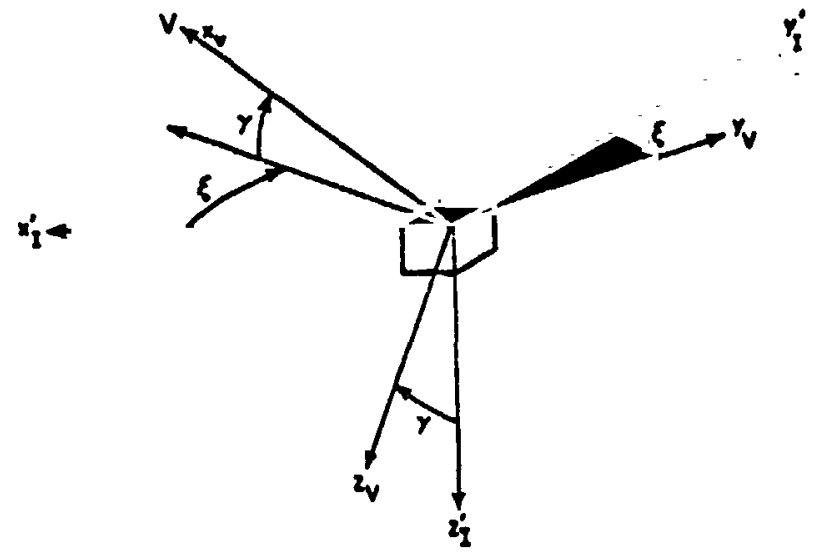

b) Inertial-Velocity Orientation

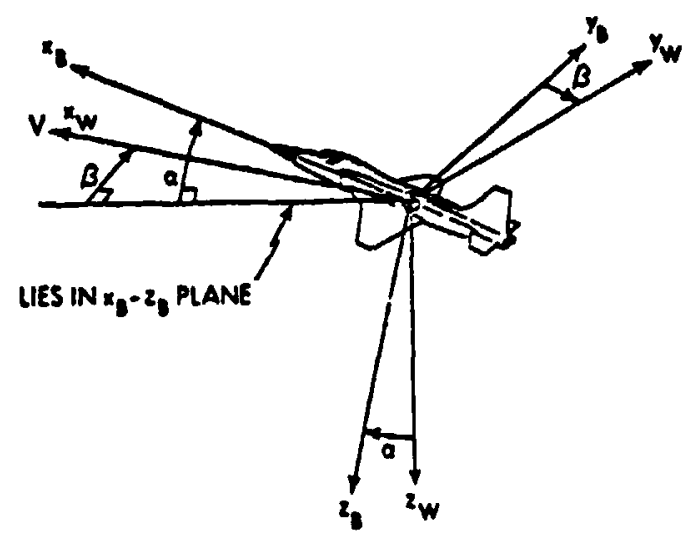

c) Wind-Body Orientation

Figure A.2-1 Reference Frame Relations 
very useful in dynamic calculations because the orientation angles between the wind frame and body frame have large influences on the aerodynamic forces and moments. Figure A.2-1c illustrates the orientation between body and wind axes. Figure A.2-2 summarizes the transformations between reference frames. Any necessary transformation can be identfied from this figure, noting that the Euler angles are given in the order of yaw, pitch, and roll, as specified by the arrows. For example, a transformation from inertial to body axes is composed of a right-handed yaw through an angle, $\psi$, then a right-handed pitch through an angle, $\theta$, and then a right-handed roll through an angle, $\phi$. These three single-angle transformations can be combined to form an inertial-body transformation as follows:

$$
\begin{aligned}
\mathrm{H}_{\mathrm{I}}^{\mathrm{B}}(\phi, \theta, \psi) & =\mathrm{H}_{2}^{\mathrm{B}}(\phi) \mathrm{H}_{1}^{2}(\theta) \mathrm{H}_{\mathrm{I}}^{1}(\psi) \\
& =\left[\begin{array}{ccc}
1 & 0 & 0 \\
0 & \cos \phi & \sin \phi \\
0 & -\sin \phi & \cos \phi
\end{array}\right]\left[\begin{array}{ccc}
\cos \theta & 0 & -\sin \theta \\
0 & 1 & 0 \\
\sin \theta & 0 & \cos \theta
\end{array}\right]\left[\begin{array}{ccc}
\cos \psi & \sin \psi & 0 \\
-\sin \psi & \cos \psi & 0 \\
0 & 0 & 1
\end{array}\right]
\end{aligned}
$$

For orthogonal matrices such as these, the matrix inverse, ()$^{-1}$, is equal to the transpose, ()$^{\mathrm{T}}$.

In the remainder of Section A.2, the vehicle's equation of motion is derived as a single state-vector equation of the form,

$$
\underline{\dot{x}}=\underline{f}(\underline{x}, \underline{u})
$$

where $\underline{x}$ is the state vector, $\underline{u}$ is the control vector, $\underline{f}$ is the vector system dynamics equation, and disturbances are neglected. The state vector is a 12 -element vector, and the nonlinear state equations are readily derived as four 


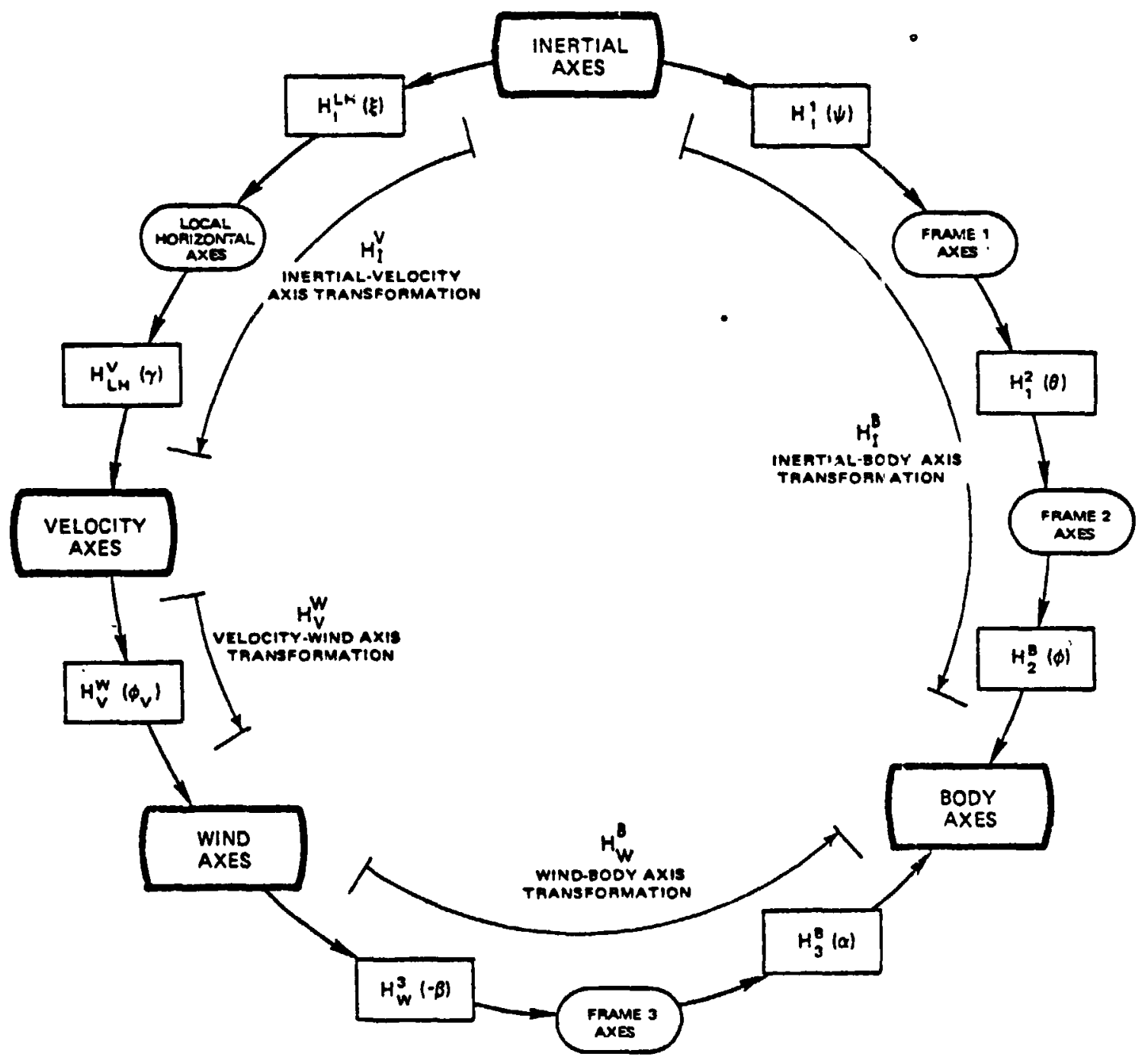

Figure A.2-2 Reference Axis Transformations (Arrows Indicate Right-Hand Rotation)

sets of three equations representing

- Translational Kinematics

- Rotational Kinematics

- Translational Dynamics

- Rotational Dynamics 
The kinematic equations relate the vehicle's translational and rotational velocities to its position in inertial space, and they involve body-axis/inertial-axis relationships. The dynamic equations describe the changes of the vehicle velocities caused by the applied forces and moments; they are best derived in a body-fixed frame of reference.

\section{A.2.1 Kinematics}

Kinematics is the study of the motion of a body without regard to the forces which cause that motion. In this section, the relations between the vehicle's position and velocity are examined. The translational and angular position of the vehicle are given relative to inertial space by the inertial position vector, $x_{I}$, and by the inertialbody Euler angle vector, $\underline{v}_{B}$ :

$$
\begin{aligned}
& \underline{x}_{I}=\left[\begin{array}{l}
x_{I} \\
y_{I} \\
z_{I}
\end{array}\right] \\
& \underline{v}_{B}=\left[\begin{array}{l}
\phi \\
\theta \\
\psi
\end{array}\right]
\end{aligned}
$$

It is important to note that the Euler angle "vector" is not a true vector in physical space; it is an ordered triple c $f$ right-handed rotations which occur about different axes of different reference frames.

The translational and angular rate vectors are often expressed in body axes, as in the following: 


$$
\begin{aligned}
& \underline{v}_{B}=\left[\begin{array}{l}
u \\
v \\
w
\end{array}\right] \\
& \underline{\omega}_{B}^{I}=\left[\begin{array}{l}
p \\
q \\
r
\end{array}\right]
\end{aligned}
$$

The body-axis translational rate vector, $\underline{v}_{B}$, is an expression, in body axes, of the derivative of the inertial position vector. This relationship supplies the first part of the nonIinear state equations of motion:

$$
\underline{\dot{x}}_{I}=H_{B}^{I} \underline{v}_{B}
$$

where $A_{B}^{I}$ is the inverse of the inertial-body transformation dertved in Eq. (A.2-1).

The body angular rate vector also can be related to the derivative of the Euler angle vector by noting that the Euler angle derivatives occur in three different reference frames. The resulting transformation 18 constructed in Eq. (A.2-8), where the individual transformations are the same as those of Fig. A.2-2 and Eq. (A.2.-1):

$$
\begin{aligned}
\underline{\omega}_{B}^{I} & =\left[\begin{array}{l}
\dot{\phi} \\
0 \\
0
\end{array}\right]+\mathbf{H}_{2}^{B}\left[\begin{array}{l}
0 \\
\dot{\theta} \\
0
\end{array}\right]+\mathbf{H}_{2}^{B_{1}^{2}}\left[\begin{array}{l}
0 \\
0 \\
\dot{\psi}
\end{array}\right] \\
& =L_{B} \underline{\dot{v}}_{B}
\end{aligned}
$$

The non-ortbogonal transformation, $I_{B}$, 18

$$
L_{B}=\left[\begin{array}{ccc}
1 & 0 & -\sin \theta \\
0 & \cos \phi & \sin \phi \cos \theta \\
0 & -\sin \phi & \cos \phi \cos \theta
\end{array}\right]
$$



where

$$
\dot{\mathrm{H}}_{\mathrm{B}}^{\mathrm{I}}=\mathrm{H}_{\mathrm{B}}^{\mathrm{I}} \tilde{\omega}_{\mathrm{B}}^{\mathrm{I}}
$$

and $\tilde{\omega}_{B}^{I}$ is the cross-product equivalent matrix for $\omega_{B}^{I}$ given by

$$
\tilde{\omega}_{B}^{I}=\left[\begin{array}{ccc}
0 & -r & q \\
r & 0 & -p \\
-q & 0 & 0
\end{array}\right]
$$

This leads to the body-axis equation;

$$
\dot{\dot{v}}_{B}=H_{I}^{B_{x_{I}}}-\tilde{\omega}_{B}^{I} \underline{v}_{B}
$$

The applied specific forces consist of gravitational forces and aerodynamic forces. The gravity force is especially simple in inertial axes, as it is confined to the vertical axis:

$$
g_{I}=\left[\begin{array}{l}
0 \\
0 \\
g
\end{array}\right]
$$

The specific contact force can be broken into two components, one of which is due to aerodynamic forces and one of which is due to thrust:

$$
\underline{F}_{B} / m=\left[\begin{array}{l}
X / m \\
Y / m \\
Z / m
\end{array}\right]
$$




$$
\underline{T}_{B} / m=\left[\begin{array}{l}
T_{B_{x}} / m \\
T_{B_{y}} / m \\
T_{B_{z}} / m
\end{array}\right]
$$

(Capital letters are conventionally used in aerodynamics to denote the force components.)

The translational dynamic equation is formed by equating the sum of the aerodynamic and gravitational specific forces to the inertial translational acceleration of the vehicle. When all vectors are expressed in body axes and the derivatives of the body-axis velocities are isolated on the left-hand side, the vector equation is

$$
\dot{\underline{v}}_{B}=\left(\underline{F}_{B}+\underline{T}_{B}\right) / m+H_{I}^{B} g_{I}-\tilde{\omega}_{B}^{I} \underline{v}_{B}
$$

To construct the rotational dynamic equation, an expression for the time derivative of angular momentum measured in inertial axes is necessary. The angular momentum, $\underline{b}_{B}$, is most easily expressed in body axes; neglecting rotatIng machinery, it is the product of the moment-of-inertia matrix (constant in body axes) and the angular rate vector,

$$
\underline{h}_{B}=I_{B} \underline{\omega}_{B}^{I}
$$

where the inertia matrix contains all products and moments of inertia:

$$
I_{B}=\left[\begin{array}{lll}
I_{x} & -I_{x y} & -I_{x z} \\
-I_{x y} & I_{y} & -I_{y z} \\
-I_{x z} & -I_{y z} & I_{z}
\end{array}\right]
$$




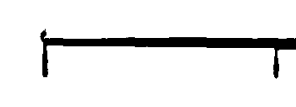

The time derivative of the angular momentum, expressed in inertial axes, is easily derived by noting that the transformation $\mathrm{H}_{B}$ is time-varying :

$$
\begin{aligned}
\dot{\underline{\underline{b}}}_{I} & =\mathrm{H}_{B}^{I} I_{B} \dot{\underline{\omega}}_{B}^{I}+\dot{\mathrm{H}}_{B}^{I} I_{B} \underline{\omega}_{B}^{I} \\
& =\mathrm{H}_{B}^{I} I_{B} \dot{\underline{\omega}}_{B}^{I}+H_{B}^{I} \tilde{\omega}_{B}^{I} I_{B} \underline{\omega}_{B}^{I}
\end{aligned}
$$

The contact moments consist of aerodynamic and thrust components. These are defined as

$$
\begin{aligned}
& \underline{M}_{B}=\left[\begin{array}{l}
L \\
M \\
N
\end{array}\right] \\
& \underline{G}_{B}=\left[\begin{array}{l}
G_{B_{x}} \\
G_{B_{y}} \\
G_{B_{z}}
\end{array}\right]
\end{aligned}
$$

(Capital letters are conventionally used for the moment components.) The rotational dynamic equation can be formed by equating the applied torques to the derivative of the angular momentum. All vectors are expressed $1:$ : body axes, and the derivative of body-axis angular rate is 1solated on the left-hand side of the equation, resulting in

$$
\dot{\underline{\omega}}_{B}^{I}=I_{B}^{-1}\left(\underline{M}_{B}+\underline{G}_{B}\right)-I_{B}^{-1} \tilde{\omega}_{B}^{I} I_{B} \underline{u}_{B}^{I}
$$

\section{A.2.3 Summary of Equations}

Section A. 2 has presented the various reference frames of interest and has deived the equations of motion of an atmospheric vehicle. The 12 -element state vector consists of three positions, three angular orientations, three 


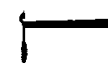

translational rates and three angular rates. The state equations were found by examininf the translational and rotational kinematics and dynamics, and are repeated here:

$$
\begin{aligned}
& \underline{\underline{z}}_{I}=H_{B}^{I} \underline{v}_{B} \\
& \dot{\underline{v}}_{B}=L_{B}^{-1} \underline{\omega}_{B}^{I} \\
& \dot{\underline{v}}_{B}=\left(\underline{F}_{B}+\underline{T}_{B}\right) / m+{ }_{H}^{B} \underline{g}_{I}-\check{\omega}_{B}^{I} \underline{v}_{B} \\
& \underline{\dot{\omega}}_{B}^{I}=I_{B}^{-1}\left(\underline{M}_{B}+\underline{G}_{B}\right)-I_{B}^{-1} \tilde{\omega}_{B}^{I} I_{B} \underline{\omega}_{B}^{I}
\end{aligned}
$$

These equations fall into the general state equation form:

$$
\underline{\dot{x}}=\underline{f}(\underline{x}, \underline{u})
$$

by defining the state vector as

$$
\underline{x}^{T}=\left[\begin{array}{lllll}
\underline{x}_{I}^{T} & \underline{v}_{B}^{T} & \underline{v}_{B}^{T} & \underline{\omega}_{B}^{I}
\end{array}\right]
$$

and noting that the alerodynamic forces and moments are functions of the states, controls, disturbances and, to some extent, the state time history. These nonlinear state equations are useful because they are general enough to allow a thorough analysis of the departure prevention problem. They are expressed in state-space form, which is notationally efficient and which makes the subsequent linearization an easily followed process.

\section{A.3 T.INEAR EQUATIONS OF MOTION}

While the nonlinear equations derived in the pre-vious section can be solved on a digital computer, they are 

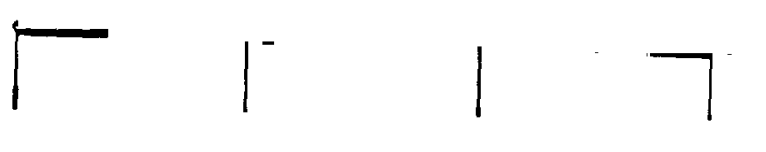

not easily analyred by general techniques, and general closed-form solutions cannot be obtained. Many of the important dynamic attributes of the aircraft can be preserved and the analysis facilitated by developing corresponding linearized equations of motion, as is done in Refs. 64 to 67 .

\section{A.3.1 Derivation from Nonlinear Equations}

The linearization procedure begins with the construction of a Taylor series expansion representing the nonlinear equations about some nominal trajectory:

$$
\underline{\dot{x}}=\dot{x}_{0}+\Delta \underline{\dot{x}}=\underline{f}\left(\underline{x}_{0}, \underline{u}_{0}\right)+\left.\frac{\partial \underline{f}}{\partial \underline{x}}\right|_{\substack{\underline{x}=\underline{x}_{0} \\ \underline{u}_{=}=\underline{u}_{0}}} \Delta \underline{x}+\left.\frac{\partial \underline{f}}{\partial \underline{u}}\right|_{\substack{\underline{x}=\underline{x}_{0} \\ \underline{u}=\underline{u}_{0}}} \Delta \underline{u}+\text { Higher Order Terms }
$$

\section{(A. 3-1)}

where the subscript "0" indicates tne nominal value and the prefix " $\Delta$ " denotes a small perturbation. All except firstorder terms are then neglected by arguing that the higherorder terms are small compared to linear terms. The results of this procedure are separatad into a nonlinear equation describing the nominal trajectory (Eq. (A.3-2)) and a linear equation defining the dynamics of the perturbations about the nominal trajectory (Eq. (A.3-3)):

$$
\begin{aligned}
& \underline{\dot{x}}_{0}=\underline{f}\left(\underline{x}_{0}, \underline{u}_{0}\right) \\
& \Delta \underline{\dot{x}}=F \Delta \underline{x}+G \Delta \underline{u}
\end{aligned}
$$




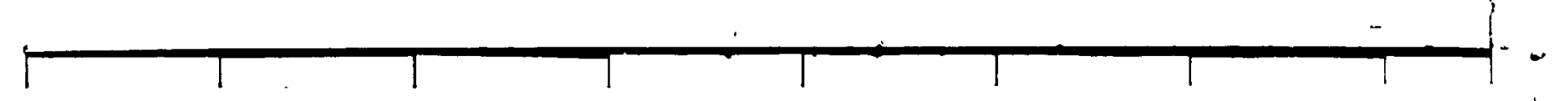

where

$$
F=\left.\frac{\partial \underline{\underline{f}}}{\partial \underline{x}}\right|_{\underline{x}=\underline{x}_{0}}
$$

and

$$
\begin{aligned}
& G=\left.\frac{\partial \underline{f}}{\partial \underline{u}}\right|_{\underline{x}=\underline{x}_{0}} \\
& \underline{u}=\underline{u}_{0}
\end{aligned}
$$

The linearization is straightforward because the nonlinear state equations (Eq. (A.2-25) to A.2-28)) are specified in a general state-space format. Equations for the perturbations of the axis transformations are easily derived by taking the partial derivatives of each transformation with respect to the Euler angles of that transformation and multiplying by the Euler angle perturbations. For the inertialbody transformution and its inverse, these transformation perturbations can be stated as follows:

$$
\Delta \mathrm{H}_{\mathrm{I}}^{\mathrm{B}}=-\Delta \tilde{\mu}_{\mathrm{B}} \mathrm{H}_{\mathrm{I}_{0}}^{\mathrm{B}}
$$

where the cross-product operator ( ) is employed,

$$
\Delta \underline{\mu}_{B}=L_{B_{0}} \underline{u}_{B}
$$

and

$$
\Delta \mathrm{H}_{\mathrm{B}}^{\mathrm{I}}=\mathrm{H}_{\mathrm{B}_{0}}^{\mathrm{I}} \Delta \tilde{\mu}_{\mathrm{B}}
$$

These relationships for the transformation perturbations are used to linearize the translational kinematic 
equation, Eq. (A.2-25), to give

$$
\Delta \underline{\dot{x}}_{I}=-(\overbrace{\dot{x}_{I_{0}}}) H_{B_{0}}^{I} \quad L_{B_{0}} \underline{v}_{B}+H_{B_{0}}^{I} \quad \underline{v}_{B}
$$

This equation clearly shows that the perturbation inertial vefocity depends both on the perturbation body-axis velocity and the inertial-body Euler angle perturbations.

The rotational kinematic equation, Eq. (A.2-26), results in the following linear perturbation equation:

$$
\Delta \underline{\dot{v}}_{\mathrm{B}}=-\mathrm{L}_{\mathrm{B}_{0}^{-1}}^{-1} \mathrm{~L}_{\mathrm{B}_{0}}^{\prime} \Delta \underline{v}_{\mathrm{B}}+\mathrm{L}_{\mathrm{B}_{0}^{-1}}^{-1} \underline{\omega}_{\mathrm{B}}^{\mathrm{I}}
$$

where

$$
\mathrm{L}_{\mathrm{B}_{0}}^{\prime}=\left.\frac{\partial\left(\mathrm{L}_{\mathrm{B}} \underline{\dot{v}}_{\mathrm{B}}\right)}{\partial \underline{-}_{\mathrm{B}}}\right|_{\underline{v}_{\mathrm{B}}=\underline{v}_{\mathrm{B}}}
$$

so that

$$
-L_{B_{0}}^{-1} L_{B_{0}}^{\prime}=\left[\begin{array}{ccc}
\dot{\theta}_{0} \tan \theta_{0} & \dot{\psi}_{0} \sec \theta_{0} & 0 \\
-\dot{\psi}_{0} \cos \theta_{0} & 0 & 0 \\
\dot{\theta}_{0} \sec \theta_{0} & \dot{\psi}_{0} \tan \theta_{0} & 0
\end{array}\right]
$$

The definition of $\mathrm{L}_{\mathrm{B}_{0}}$ takes the form it does because the linear rotational kinematic equation was derived from the original form of the rotational kinematics equation, given in Eq. (A.2-8).

Linearization of the dynamic equations requires consideration of the aerodynamic force and moment relationships. These are functions of the states, controls, and the past history of these variables. This dependence on 
past values is caused by aerodynamic flow field effects and their propagation delays; unsteady aerodynamic effects can be represented as functions of the state rates. The formal linearization of the aerodynamic forces and moments is a lengthy but straightf.rward process which amounts to taking the partial derivatives of every aerodynamic force and moment vector with respect to the states, state rates, and controls. These partial derivatives are called stability derivatives.

The difficulty revolves around the actual values to be used for each of these coefficients. This data is produced primarily by wind tunnel testing, as described, for example, in Refs. 68 and 69 . There is a large amount of effort and expense involved in generating this data, so only the most important functional relationships can be examined. This often results in different data sets for each aircraft. For this reason, only general terms for the perturbation forces and moments are included in the following discussion. .ppendix $B$ contains a discussion and example of the construction of force and moment stability derivative matrices from real data. Many stability derivative matrices are either known to be zero or are so small as to be neglected in all cases of interest. Assuming that altitude and orientation variations have negligible effect on contact force and moment variations, and assiming insignificant state derivative and angular rate effects on thrust forces and moments, the perturbation aerodynamic forces and moments are as follows:

$$
\Delta \underline{F}_{B}=\left[\frac{\partial \underline{F}_{B}}{\partial \underline{v}_{B}}\right] \Delta \underline{v}_{B}+\left[\frac{\partial \underline{F}_{B}}{\partial \underline{\omega}_{B}}\right] \Delta \underline{\omega}_{B}^{I}+\left[\frac{\partial \underline{F}_{B}}{\partial \underline{u}^{\prime}}\right] \Delta \underline{u}^{\prime}+\left[\frac{\partial \underline{F}_{B}}{\partial \dot{\dot{v}}_{B}}\right] \Delta \underline{\dot{v}}_{B}+\left[\frac{\partial \underline{F}_{B}}{\partial \underline{\dot{\omega}}_{B}^{I}}\right] \Delta \underline{\dot{\omega}}_{B}^{I}
$$




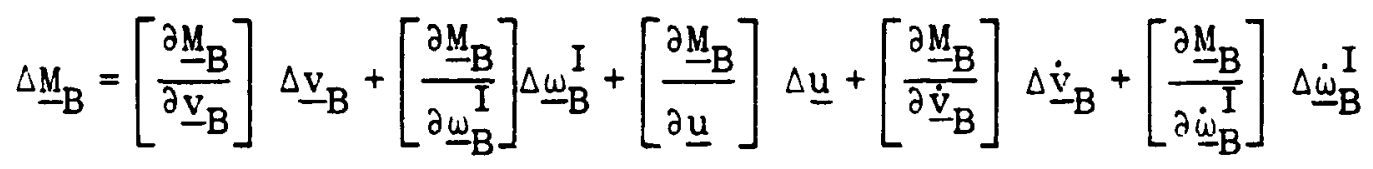

$$
\Delta \underline{T}_{B}=\left[\frac{\partial \underline{T}}{\partial \underline{v}_{B}}\right] \Delta \underline{v}_{B}+\left[\frac{\partial \underline{T}}{\partial \underline{\mathrm{u}}}\right] \underline{\Delta u}
$$

$\Delta \underline{G}_{B}=\left[\frac{\partial \underline{G}_{B}}{\partial \underline{v}_{B}}\right] \Delta \underline{v}_{B}+\left[\frac{\partial \underline{G}_{B}}{\partial \underline{u}}\right] \underline{\Delta u}$

The linear translational dynamic equation is derived from Eq. (A 2-27) and incorporates the perturbation aerodynamic force expressions presented above. Note that the state-rate stability derivative matrices enter the equation in a different manner than the other terms; they must be moved to the left-hand side of the dynamic equations. The linear translational dynamic equation becomes

$$
\begin{aligned}
& \left\{I-\frac{1}{m}\left[\frac{\partial \underline{F}_{B}}{\partial \dot{\underline{v}}_{B}}\right]\right\} \Delta \dot{\underline{\dot{v}}}_{B}+\left\{-\frac{1}{m}\left[\frac{\partial \underline{\underline{F}}_{B}}{\partial \underline{\underline{\omega}}_{B}^{I}}\right]\right\} \underline{\underline{\dot{\omega}}}_{B}^{I}=\tilde{\mathrm{g}}_{B_{0}} L_{B_{0}} \Delta \underline{v}_{B} \\
& +\left\{\frac{1}{m}\left[\frac{\partial \underline{F}_{B}}{\partial \underline{v}_{B}}\right]+\frac{1}{m}\left[\frac{\partial \underline{T}_{B}}{\partial \underline{v}_{B}}\right]-\tilde{\omega}_{B}^{I}\right\} \Delta \underline{v}_{B} \\
& +\left\{\frac{1}{m}\left[\frac{\partial \underline{F}_{B}}{\partial \underline{\omega}_{B}^{I}}\right]+\tilde{v}_{B}\right\} \underline{\omega}_{B}^{I} \\
& +\left\{\frac{1}{m}\left[\frac{\partial \underline{F}_{B}}{\partial \underline{u}}\right]+\frac{1}{m}\left[\frac{\partial \underline{T}_{B}}{\partial \underline{u}}\right]\right\} \Delta \underline{u}
\end{aligned}
$$

$($ A. 3-17) 
The linear rotational dynamic equation is derived similarly, and the state-rate stability derivatives appear in the same way. The linear rotational dynamic equation is

$$
\begin{aligned}
& \left\{-I_{B}^{-1}\left[\frac{\partial \underline{\underline{M}}_{B}}{\partial \underline{\underline{v}}_{B}}\right]\right\} \underline{\Delta}_{B}+\left\{I-I_{B}^{-1}\left[\frac{\partial \underline{M}_{B}}{\partial \underline{\dot{\omega}}_{B}^{I}}\right]\right\} \Delta \underline{\dot{\omega}}_{B}^{I}=I_{B}^{-1}\left(\left[\frac{\partial \underline{M}_{B}}{\partial \underline{v}_{B}}\right]+\left[\frac{\partial \underline{G}_{B}}{\partial \underline{v}_{B}}\right]\right\} \Delta \underline{v}_{B} \\
& +I_{\mathrm{B}}^{-1}\left|\left[\frac{\partial \underline{\underline{M}}_{\mathrm{B}}}{\partial \underline{\omega}_{\mathrm{B}}^{I}}\right]+\widetilde{I}_{\mathrm{B}} \underline{\omega}_{\mathrm{B}_{0}}^{I}-\tilde{\omega}_{\mathrm{B}}^{I} I_{\mathrm{B}}\right| \Delta \underline{\omega}_{\mathrm{B}}^{I} \\
& +I_{B}^{-1}\left\{\left[\frac{\partial \underline{u}_{B}}{\partial \underline{u}}\right]+\left[\frac{\partial \underline{G}_{B}}{\partial \underline{u}}\right]\right\} \Delta \underline{u}
\end{aligned}
$$

The four state equations (translational and rotational kinematic and dynamic equations) can be written in standard linear equation form ( $\mathrm{Eq} .(\mathrm{A} .3-3)$ ) by using the following state vector:

$$
\Delta \underline{x}^{T}=\left[\begin{array}{llll}
\Delta \underline{x}_{I}^{T} & \Delta \underline{v}_{B}^{T} & \Delta \underline{v}_{B}^{T} & \Delta \underline{w}_{B}^{I}
\end{array}\right]
$$

The state equations then fall into the form:

$$
J_{B} \Delta \underline{\dot{x}}_{B}=F_{B}^{\prime} \Delta \underline{x}_{B}+G_{B}^{\prime} \Delta \underline{u}
$$

where the state-rate transformation matrix is

$$
J_{B}=\left[\begin{array}{cccc}
I & 0 & 0 & 0 \\
0 & I & 0 & 0 \\
0 & 0 & J_{33} & J_{34} \\
0 & 0 & J_{43} & J_{44}
\end{array}\right]
$$


and the three-by-three sub-matrices are

$$
\begin{aligned}
& J_{33}=I-\frac{1}{m}\left[\frac{\partial \underline{F}_{B}}{\partial \underline{\dot{v}}_{B}}\right] \\
& J_{34}=-\frac{1}{m}\left[\frac{\partial \underline{F}_{B}}{\partial \underline{\dot{\omega}}_{B}^{I}}\right] \\
& J_{43}=-I_{B}^{-1}\left[\frac{\partial \underline{M}_{B}}{\partial \underline{\hat{v}}_{B}}\right] \\
& J_{44}=I-I_{B}^{-1}\left[\frac{\partial \underline{M}_{B}}{\partial \dot{\omega}_{B}^{I}}\right]
\end{aligned}
$$

The primed state dynamics matrix is

$$
F_{B}^{\prime}=\left[\begin{array}{cccc}
0 & F_{12} & F_{13} & 0 \\
0 & F_{22} & 0 & F_{24} \\
0 & F_{32} & F_{33} & F_{34} \\
0 & 0 & F_{43} & F_{44}
\end{array}\right]
$$

where the three-by-three sub-matrices are

$$
\begin{aligned}
& F_{12}=-\tilde{\dot{x}}_{I_{0}} H_{B_{0}}^{I} L_{B_{0}} \\
& F_{13}=H_{B_{0}}^{I} \\
& F_{22}=-L_{B_{0}}^{-1} L_{B_{0}} \\
& F_{24}=L^{-1}
\end{aligned}
$$




$$
\begin{aligned}
& F_{32}=\mathrm{H}_{\mathrm{I}_{0}}^{\mathrm{B}} \tilde{\mathrm{g}}_{\mathrm{I}} \mathrm{H}_{\mathrm{B}_{0}}^{\mathrm{I}} \mathrm{L}_{\mathrm{B}_{0}} \\
& F_{33}=\left\{\frac{1}{m}\left[\frac{\partial F_{B}}{\partial \underline{v}_{B}}\right]+\frac{1}{m}\left[\frac{\partial T}{\partial \underline{v}_{B}}\right]-\tilde{\omega}_{B_{0}}^{I}\right\} \\
& F_{34}=\left|\frac{1}{m}\left[\frac{\partial \underline{F}_{B}}{\partial \underline{\omega}_{B}^{I}}\right]+\tilde{v}_{B_{0}}\right| \\
& F_{43}=I_{B}^{-1}\left\{\left[\frac{\partial \underline{M}_{B}}{\partial \underline{v}_{B}}\right]+\left[\frac{\partial \underline{G}_{B}}{\partial \underline{v}_{B}}\right]\right\} \\
& F_{44}=I_{B}^{-1}\left\{\left[\frac{\partial \underline{M}_{B}}{\partial \underline{\omega}_{B}^{I}}\right]+\widetilde{I_{B} \underline{\omega}_{B}^{I}}-\tilde{\omega}_{B_{0}}^{I} I_{B}\right\}
\end{aligned}
$$

and

$$
G_{B}^{\prime}=\left[\begin{array}{c}
0 \\
0 \\
G_{3} \\
G_{4}
\end{array}\right]
$$

The sub-matrices (of three rows and as many columns as controls) are

$$
\begin{aligned}
& G_{3}=\left\{\frac{1}{m}\left[\frac{\partial \underline{F}_{B}}{\partial \underline{u}}\right]+\frac{1}{i n}\left[\frac{\partial \underline{T}}{\partial \underline{u}}\right]\right\} \\
& G_{4}=\left\{I_{B}^{-1}\left[\frac{\partial \underline{M}}{\partial \underline{u}}\right]+I_{B}^{-1}\left[\frac{\partial \underline{G}_{B}}{\partial \underline{u}}\right]\right\}
\end{aligned}
$$

The complete state equation is produced by premultiplying Eq. (A.3-20) by the inverse of the state-rate 
transformation matrix, giving the following result:

$$
\begin{aligned}
\Delta \dot{x}_{B} & =J_{B}^{-1} F_{B}^{\prime} \Delta \underline{x}_{B}+J_{B}^{-1} G_{B}^{\prime} \Delta \underline{u} \\
& =F_{B} \Delta \underline{x}_{B}+G_{B} \Delta \underline{u}
\end{aligned}
$$

This resulting linear system is analyzed throughout this report. It is important to note that this system specifies the state and control perturbations about their nominal values. Methods of properly choosing these nominal values are examined next.

\section{A. 3.2 Generalized Trim Conditions}

An aircraft is in the trimmed condition when its controls are set to produce equilibrium in the equations of motion. Steady trim occurs when the aircraft is under no inertial acceleration, i.e., when translational velocities are constant and rotational rates are zero. The trim concept can be extended to dynamic flight conditions by defining generalized trim as the condition in which control settings produce constant velocities and angular rates. In this case, the vehicle is not necessarily in steady equilibrium, due to changing roll and pitch angles.

The importance of these trim classifications lies in the use of trimmed flight conditions as nominal trajectories for linearization, since trim implies that the nominal velocities, angular rates, and controls are constant, or, at most, slowly varying. Thus, the use of the trimmed condition as a nominal flight condition causes the linear equations to represent almost all of the system dynamics at that flight condition. This can be seen by witing the general equations for the total state rate: 


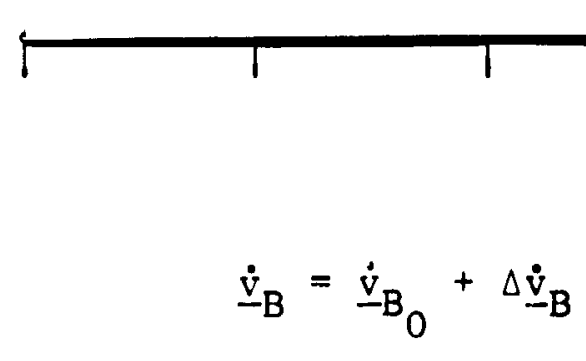

$$
\begin{aligned}
& \dot{\omega}_{B}^{I}=\dot{\dot{\omega}}_{B}^{I}+\Delta \underline{\dot{\omega}}_{\mathrm{B}}^{I}
\end{aligned}
$$

If a set of nominal states and controls can be found so that the generalized trim condition

$$
\dot{\dot{v}}_{\mathrm{B}_{0}}=\dot{\dot{\omega}}_{\mathrm{B}_{0}}^{\mathrm{I}}=0
$$

is satisfied, then the perturbation state rates are equal to the total state rates. This can be seen by inserting Eq. (A.3-42) into Eqs. (A.3-40) and (A.3-41).

Another desirable characteristic of using the trimmed flight condition as a nominal for linearization is that the total state and control trajectories over a significant interval of time are the sums of the constant nominal values and the linear perturbation time histories. This relation is given as:

$$
\begin{aligned}
& \underline{v}_{B}(t) \cong \underline{v}_{B}+\Delta \underline{v}_{B}(t) \\
& \underline{\omega}_{B}^{I}(t) \cong \underline{\omega}_{B_{0}}^{I}+\Delta \underline{\omega}_{B}^{I}(t) \\
& \underline{u}(t) \cong \underline{u}_{0}+\Delta \underline{u}^{\prime}(t)
\end{aligned}
$$

where $\underline{v}_{B_{0}} \cdot \underline{\omega}_{B_{0}}^{I}$ and $\underline{u}_{0}$ are constant. 
A solution method for the generalized trim problem can be derived by examining general forms of tise nunisicar translational and rotational dynamic equ tions, which are derived in Sect, $n$ A.2.2 as

$$
\begin{aligned}
& \dot{\underline{v}}_{B}=\underline{f}_{-1}\left(\underline{v}_{B}, \underline{v}_{B}, \underline{\omega}_{B}^{I}, \underline{u}\right) \\
& \underline{\dot{\omega}}_{B}^{I}=\underline{f}_{2}\left(\underline{v}_{B}, \underline{\omega}_{B}^{I}, \underline{u}\right)
\end{aligned}
$$

The aerodynami: forces and moments are assumed to be functions of $\underline{v}_{B}, \underline{w}_{B}$, and $\underline{u}$. One element of the Euler angle vector, $\psi$, does not appear in the equations, and altitude is a parameter.

Two different generalized trim problems then become possible, the first of which is the following:

- Find the values of velocity and angular rate ( $\underline{v}_{B}$ and $\underline{\omega}_{B}^{I}$ ) that produce generalized trim $\left(\underline{\dot{v}}_{B}=\underline{\dot{\omega}}_{B}^{I}=0\right)$ for given controls and Euler angles $\left(\underline{u}, \underline{v}_{B}\right)$.

This problem consists of six equations (the dynamic equations) in six uninuwns (the velocity and angular rates), and therefore it can ie expected to have a solution. The controls and Euler angles are the set points that determine the generalized trim solution, and it should be noted that altitude also has an effect on the solution.

The second generalized trim problem can provide the trim controls that produce specific state values:

- Find the values of the controls and Euler angles ( $\underline{u}$ and $\underline{v}_{B}$ ) tiat satisfy the generalized trim conditions ( $\left.\dot{\underline{v}}_{B}=\underline{\dot{w}}_{B}^{I}=0\right)$ for riven val les of the velocity and ancular rate vertors ( $\underline{\mathrm{V}}_{\mathrm{B}}$ and $\underline{\omega}_{\mathrm{B}}^{\mathrm{I}}$ ). 
The existence of a solution to the second problem depends on the degrees of freedom and power of the controls. Many aerodynanic vehicles have a four-element control vector; along with the Euler an;les, $D$ and $\theta$, this results in a problem of six equations (the dynamic equations) in six unkinowns (throttle, elevator, aileron, rudder, pitch angle, and roll angle).

One approach for sclving either of these generalized trim problems is to use functional minimization. This approach, also called parametric optimization, requires all elements of $\underline{u}$ and $\underline{v}_{B}\left(O ت\right.$ of $\underline{v}_{-B}$ and $\left.\underline{v}_{B}\right)$ to be specified as given or desired, but it : s not tied to any particular flight path. In this case. Eqs. (A.3-46) and (A.3-47) are solved directly using an iterative process, e.g., a steepest-descent, accelerated gradient algorithm, or direct numerical search (Ref. 70). A scalar cost function, J, measures trim error; a quadiatic form is appropriate for computing a norm of the vector error:

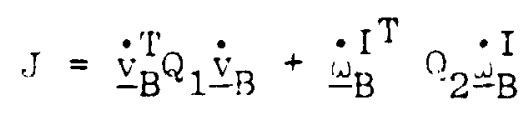

In both generalized trim problems, the trim value is determined when $J$ reaches a minim....

\section{A.3.3 Body-Axis Equations}

It is advantageous to express the vehicle state equations in oody-fixed axes. These are the axes in which the pilot, the sensors, and the control surfaces are located. Budy axas are thr only axes in which the moment-of-inertia matrix is constant. Aerodynamic data collected from stingmounted wind tanf lodels or from tleght test usually is 
expressed in body axes. Consequently, body axes are considered to be the basic axes in this report, and all of the equations given so far have been in body axes.

\section{A.3.4 Staility-Axis Equations}

Stability axes also are body-fixed axes, so that they retain the convenient characteristics mentioned above. The axes are fixed in the body so that the $x$-axis is aligned with the nominal velocity vector. Although the axes are fixed in the body, they have different orlentations at different nominal flight conditions. Further, the perturbation velocity vector is not expressed as three orthogonal velocity perturbations but as a velocity magnituc perturbation and two body-velocity orientation ansles: perturbation angles of attack $(\Delta \alpha)$ and sideslip $(\triangle B)$. This rector is referred to here as $\underline{\Delta}_{W}$. It is important to note that stability axe's are also the same as wind axes for a specific nominal flight condition: $\dot{\alpha}_{0}=\dot{B}_{0}=0$. This is the justification for usirg a "W" subscript throughout this report for stability axis variables.

Stability axes simplify certain aspects of the linear equations. In level nominal flight, stability $x-$ and $y$-axes are horizontal and the z-axis is vertical. The lift and drag forces act along statility axes. Finally, it often is true that stabiilty axes are close to the normal mode axes. This means that the basic modes of motion appear as motion about or along a single axis of the stability reference frame. The frequency and damping of the basic modes then become simple functions of the aerodynamic stability derivatives expressed along stability axes; therefore aerodynamic stability derivatives expressed in the stability-axjs frame can be used as approximate indications of stahility or instabllity. For example, assuming that 
body axes coincide with principal axes, the stability-axis rotational dynamic equations appear in part as:

$$
\begin{aligned}
& \Delta \dot{\mathrm{p}}_{\mathrm{W}}=\frac{1}{2} \rho \mathrm{v}_{0}^{2} \mathrm{Sb} \frac{1}{\overline{\mathrm{I}}_{\mathrm{x}}}\left[\cos \alpha_{0} \mathrm{C}_{\mathrm{I}_{B}}+\frac{\mathrm{I}_{\mathrm{x}}}{\mathrm{I}_{z}} \sin \alpha_{0} \mathrm{c}_{\mathrm{n}_{B}}\right] \Delta \varepsilon+\ldots \\
& \Delta \dot{\mathrm{r}}_{W}=\frac{1}{2} \rho v_{0}^{2} \mathrm{Sb} \frac{1}{\mathrm{I}_{z}}\left[\cos \alpha_{0} c_{\mathrm{n}_{\beta}}-\frac{\mathrm{I}_{z}}{\mathrm{I}_{\mathrm{X}}} \sin \alpha_{0} c_{1_{\beta}}\right] \Delta \beta+\ldots
\end{aligned}
$$

where Appendix B details the definition of the individual symbols. The departure parameter $\mathrm{C}_{\mathrm{n}_{B} \text {, dyn }}$ (Section 2.2 ) is recognized as an element in the yaw equation. This is significant because these equations are derived by a simplification of the complete equations, and it is possible that other useful departure parameters can be derived from the same approach.

Since stability axes are body-fixed axes with a particular nominal orientation, it is convenient to derive the linear equ tiuns in body axes and simply rotate them to obtain a stability-axis set. This can be done by applying the transformation matrix, $K_{B}$, to the body-axis system matrices, $F_{B}$ and $G_{B}$, as follows:

$$
\begin{aligned}
& F_{W}=K_{B W}^{-1} F_{B} K_{B W} \\
& G_{W}=K_{B W}^{-1} G_{E}
\end{aligned}
$$

where

$$
\mathrm{K}_{\mathrm{BW}}=\left[\begin{array}{cccc}
\mathrm{I} & 0 & 0 & 0 \\
0 & \mathrm{I} & 0 & 0 \\
0 & 0 & \mathrm{HW}_{0}^{\mathrm{B}}{ }^{S_{W_{0}}} & 0 \\
0 & 0 & { }^{\mathrm{H}}{ }_{\mathrm{B}_{0}}{ }^{L_{W_{0}}} & \mathrm{H}_{W_{0}}^{\mathrm{B}}
\end{array}\right]
$$


and, from Fig. 2.2-2, the wind-body transformation is

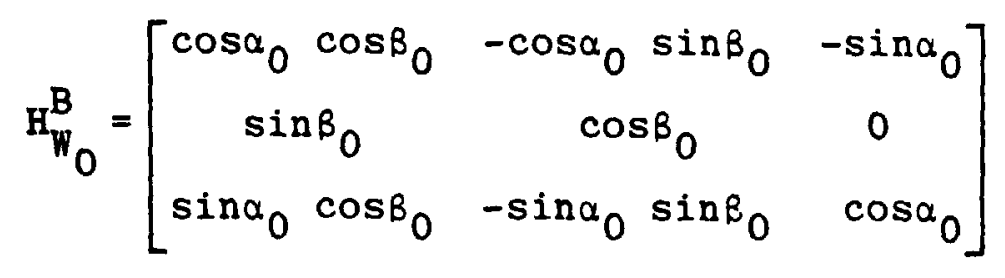

while

$$
L_{W_{0}}=\left[\begin{array}{ccc}
0 & \sin \alpha_{0} & 0 \\
0 & 0 & 1 \\
0 & -\cos \alpha_{0} & 0
\end{array}\right]
$$

and $J_{W_{0}}$ is a diagonal matrix whose elements are $\left[1, V_{0}, v_{0} \cos \beta_{0}\right]$. These relations (which assume $\alpha_{0}=\beta_{0}=0$ ) are used to transform body-axis equations to stability-axis equations for further analysis.

\section{A.3.5 State Ordering and Dimension}

The order of the states given by Eq. (A.2-30) is the one that proceeds from considering translation before rotation and kinematics before dynamics. This order does not, however, group related states together. For example, many aircraft demonstrate a natural mode that is primarily composed of $\Delta u$ and $\Delta \theta$ oscillations (the phugoid mode), and it is logical to regroup the states so that $\Delta u$ and $\Delta \theta$ fall next to each other.

For some reference flight conditions, a further major division between longitudinal and lateral-directiona: variables can be made. The former variables describe notion withi. the vehicle plane of symmetry, while the lateraldirectional variables describe motion out of the plane of symmetry. This division is useful because it allow's a quick appraisal or the extent and nature of cross couplings that arise in maneuvering flight. 
With this condition in mind, the ordering of states given in Table A.3-1 is suggested for aircraft and similar vehicles. The six longitudinal states are first, followed by the six lateral-directional states.

TABLE A.3-1

STATE ORDERING

\begin{tabular}{|c|c|}
\hline Body Axes & Stability Axes \\
\hline$\Delta x_{I}$ & $\Delta x_{I}$ \\
$\Delta z_{I}$ & $\Delta z_{I}$ \\
$\Delta \theta$ & $\Delta \theta$ \\
$\Delta u$ & $\Delta V$ \\
$\Delta q$ & $\Delta q_{W}$ \\
$\Delta w$ & $\Delta \alpha$ \\
$\Delta v$ & $\Delta \beta$ \\
$\Delta r$ & $\Delta r_{W}$ \\
$\Delta p$ & $\Delta p_{W}$ \\
$\Delta \phi$ & $\Delta \phi$ \\
$\Delta \psi$ & $\Delta \psi$ \\
$\Delta y_{I}$ & $\Delta y_{I}$ \\
\hline
\end{tabular}

In any reduced-order approximation, this state ordering also is useful. The four "outermost" states, for example, do not affect the inner eight, but are merely integral functions of them. Thus, $\Delta \mathbf{x}_{I}, \Delta z_{1}, \Delta y_{I}$ and $\Delta \psi$ can be removed without changing the basic modes of motion of the vehicle. The first two of the remaining eight states are the primary states involved in the phugaid mode, while the next two represent the primary short pericd longitudinal mode. The four lateral-directional states often exhibit a Iutch roll oscillatory mode and roll and spiral convergence modes.

Except in special cases, each of these modes involves most of the lateral-directional states. 

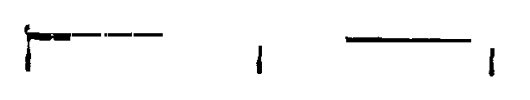

The formation of models of order less than eight depends on an examination of the individual problem. For example, wher there is no coupling between lateral-directional and longitudinal modes, the eighth-order model can be split into two independent fourth-order models with no luss of accuracy. If the time-span of interest is short, it may be possible to neglect the slower modes (phugoid mode and spiral convergence) without decreasing the accuracy of the results; however, as the eigenvectors of earlier chapters show, one runs the risk of missing significant coupling effects when "inner eight" states are eliminated.

A different order reduction suggests itself when the generalized trim problem is examined. As discussed in Section A.3.2, the goal of the generalized trim procedure is to find a nominal flight condition with constant velocity and angular rates. Because they do not affect the velocity and angular rate states, $x_{I}, y_{I}, z_{I}$ and $\psi$ are dropped immediately. To completely control the six desired states, six controls are necessary, but most atmospheric flight vehicles have less than six control effectors. Noting that the two Euler angles, $\theta$ and $\phi$, are involved primarily in the slow modes, these two states may be regarded as parameters. This results in a problem of four controi throttle, elevator, aileron, rudder), two parameters $(t, \ldots)$, six states $(u, v, w, p, q, r)$, and six state equations $(\dot{u}, \dot{v}, \dot{w}, \dot{p}, \dot{q}, \dot{r})$ to define trim. 


\section{A.4 TOOLS FOR LINEAP ANALYSIS OF AIRCRAFT STABILITY AND CONTROL}

The previous sections of this chapter have developed the linear aircraft model and demonstrated its validity along lighly dynamic trajectories. The use of a linear model is desirable because the large body of theory and experience relating to the analysis and control of linear systems then can be applied to the departure prevention problem. These tools are discussed in this section.

\section{A.4.1 Eigenvalues, Eigenvectors, and Normal Modes}

The initial-condition response of a linear-timeinvariant system is composed of a linear combination of a limited number of natural, or normal modes. Each normal mode is characterized by its time scale, given by the eigenvalue of that mode, and the relative involvement of each state in that mode, indicated by the eigenvector of that mode. In physically realizable systems, the modes are described either by individual real eigenvalues or by pairs of complex eigenvalues. A first-order mode exhibits either an exponentially increasing response (fositive eigenvalue) or an exponentially decreasing response (negative eigenvalue). A complex (second-order) mode oscillates at a frequency determined by the imaginary part of the $\in$ igenvalue within an exponential envelope determined by the real part of the eigenvalue. Therefore, the oscillation can diverge, converge, or maintain constant amplitude.

As an example of the eigenvalues involved in the normal modes of fighter aircraft, Fig. A.4-1 illustrates tho areas in the complex plane which contain the five modes anc eight basic eigenvalues of a small high-performance 


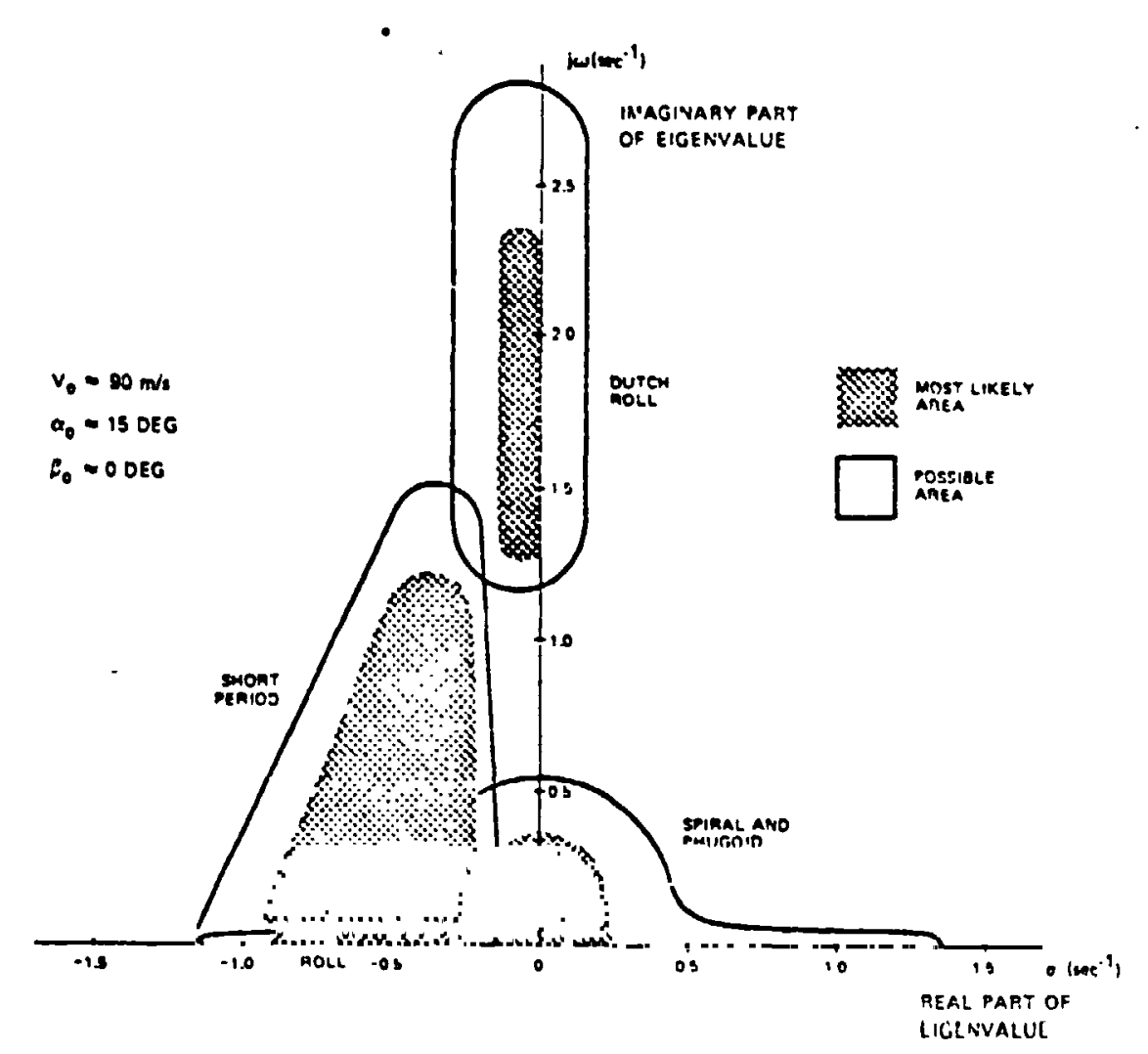

Figure A.4-1 Approximate Root Locations of a High-Performance Aircraft

aircraft. Because complex eigenvalues occur in pairs of complex conjugates, the lower half of the complex plane is symmetric with the upper half and is not shown.

The eigenvectors indicate the relative involvement of the aircraft states in a given mode. Each of the modes shown in Fig. A.4-1 may involve the motion of every state, but the following generalizations can be made for straightand-level-flight. The longitudinal states $(\Delta \theta, \Delta u, \Delta q, \Delta w)$ exhibit two second-order modes. The phugoid mode is a slow, lightly damped interchange of kinetic energy (speed) and potential energy (altitude) and primarily involves $\Delta \theta$ and $\Delta u$. The short period mode is the rapid, well-damped angular oscillation, and is exhibited primarily by $\Delta q$ and $\Delta w$. 
The lateral-directional modes usually consist of a second-order and two first-order modes. The former is called the Duteh roll mode and is a fast, poorly damped yaw oscillation about the stability $z$-axis. The roll convergence mode is a fast, stable mode, and it represents the aircraft response (generally about the stability $x$-axis) to a roll moment. Due to the angle between body and stability axes, the Dutch roll mode and the roll convergence modes appear in the three states $\Delta v, \Delta r$, and $\Delta p$. The spiral mode is slow and frequently unstable. An unstable spiral mode is important in a piloted aircraft only if the time constant is so short that the pilot has difficulty keeping the wings level.

These mode shapes change considerably as the flight condition varies from straight-and-level flight. Asymmetric flight conditions result in coupled longitudinal/lateraldirectional modes, and angular rates cause large changes in the eigenvalues. In certain cases, modes combine: the roll and spiral modes can form a roll-spiral oscillation, for example.

The eigenvalues of the outer four states $\left(\Delta x_{I}, \Delta y_{I}\right.$, and $\Delta \psi$ ) are zero, i.e., these states have no effect on the other variables and are pure integrations of the other state variables. As a consequence, control-loop closures have no direct effect on these modes unless the outer va:iables are fed back directly.

The eigenvalues are the roots of the characteristic equation of the system dynamics matrix, F,

$$
|\lambda I-F|=0
$$

where I is the identity matrix of the same order as the system matrix, and $\lambda$ is a scalar which must equal an eigenvalue for Eq. (A.4-1) to be satisfied. 
As shown in Ref. 71 , there is a set of vectors associated with the eigenvalues which have special.properties. The eigenvectors, $\underline{z}_{i}$, art linear combinations of the elements of the state vector and are solutions to the equations

$$
\left[\lambda_{i} I-F\right] \underline{z}_{i}=0
$$

As in the case of eigenvalues, the eigenvectors of a secondorder mode appear as complex conjugate pairs. The eigenvectors contain the same information about the normal modes that is given by the time vectors of classical aircraft stability analysis (Ref. 65).

The modal matrix is the matrix of eigenvectors arranged columnwise. The inverse of this matrix transforms the state vector into normal mode space, in which each element of the vector, $\Delta y$, represents a normal mode of the system:

$$
\Delta \underline{y}=M^{-1} \Delta \underline{x}
$$

(Instead of the two complex-conjugate eigenvectors of a second-order mode, it may be useful to use two real vectors, one composed of the eigenvector real part and one the imaginary part.)

The linear equation of motion, Eq. (A.3-3) can be transformed as well:

$$
\Delta \underline{\dot{y}}=M^{-1} F M \Delta \underline{y}+M^{-1} G \Delta \underline{u}
$$

The normal-mode system matrix, $M^{-i} F M$, is composed of firstor second-order diagonal blocks containing the system eigenvalues, and the normal mode input matrix, $M^{-1} G$, indicates which inputs affect which normal modes. This alternate form 
of the state equation is useful because it demonstrates a method of analyzing normal mode excitation. The excitation due to the state initial condition can be calculated from Eq. (A.4-3), while the excitation due to control inputs is given by the normal mode input matrix, $M^{-1} G$.

This section has discussed eigenvalue and eigenvector concepts. Examples of the application of these analytical tools to the analysis of linearized afrcraft models are contained in Sections 2.4 and 2.5 .

\section{A.4.2 Controllability}

In a multi-input, multi-output system, certain normal modes may be unaffected by the system controls without this being apparent from the system dynamics and input matrices. This can not occur in an $n^{\text {th }}$-order linear-timeinvariant system whose controllability test matrix, $\Gamma$, has full rank:

$$
\Gamma=\left[\begin{array}{l:l:l:l}
G & F G & \cdots & F^{n-1} G
\end{array}\right]
$$

The presence of controliability is necessary for the construction of a complete system controller, and this property almost always exists in physical systems of interest. (Controllability tests show that the high-performance fighter investigated in this report is controllable throughout the range of flight conditions.)

$$
\text { Of more interest is the investigation of control }
$$
effectiveness throughout the flight regime. The difficulty is in devising a simple measure of control effectiveness, but this can be overcome, to some extent, by using the normal mode control input matrix, $\mathrm{M}^{-1} \mathrm{~g}$, which was introduced above. The rows of this matrix indicate the relative 
importance of the aircraft's controls in affecting each of the normal modes.

\section{A.4.3 Transfer Functions}

Specific input-output relationships in linear-timeInvariant dynamic systems can be described by transfer functions, which are typically given as ratios of polynomials in the Laplace operator, $s$. The Laplace transform of the ordinary differential equation of motion ( $\mathrm{Eq} .(\mathrm{A} .3-3)$ ) is

$$
(s I-F) \Delta \underline{x}(s)=G \underline{u}(s)
$$

where $I, F$, and $G$ have been defined, and $\Delta \underline{x}(s)$ and $\Delta \underline{u}(s)$ are Laplace transforms of the state and control vectors, $\Delta \underline{x}(t)$ and $\underline{\Delta}(t)$. The input, $\underline{\Delta} \underline{u}(s)$, and the output, $\underline{\Delta} \underline{x}(s)$, are related by a transfer function matrix, $H(s)$, which is obtalned when Eq. (A.4-6) is pre-multiplied by the inverse of $(8 I-F)$ :

$$
\begin{aligned}
L \underline{x}(s) & =(s I-F)^{-1} G \underline{\Delta u}(s) \\
& =H(s) \underline{\Delta} \underline{u}(s)
\end{aligned}
$$

where

$$
\mathrm{A}(\mathrm{s})=(s I-F)^{-1} \mathrm{G}
$$

Any scalar transfer function of intertst (for example, the effect of the $i^{\text {th }}$ control on the $f^{\text {th }}$ motion variable), can be obtained from Eq. (A.4-8) by evaluating two determinants derived from the matrices of $\mathrm{Eq} .(\mathrm{A} .4-6)$ (Ref. 72),

$$
\frac{\Delta x_{j}(s)}{\Delta u_{i}(8)}=\frac{\left|s I-F+g_{1 j}\right|-|s I-F|}{|s I-F|}
$$


where $\mathbf{g}_{1 j}$ is an $\mathbf{n}$ by $\mathbf{n}$ matrix whose elements are zero, except for the $j^{\text {th }}$ column, which coniains the $i^{\text {th }}$ coiumn of the $G$ matrix. This transfer function is a ratio of polynomials in $s$, and the numerator and denominator can be factored to identify the poles, $p$, and zeros, $z$, which describe the relationship between $\Delta u_{i}(s)$ and $\Delta x_{j}(s)$ :

$$
\frac{\Delta x_{j}(s)}{\Delta u_{i}(s)}=\frac{K_{I}\left(s-z_{1}\right)\left(s-z_{2}\right) \ldots\left(s-z_{m}\right)}{\left(s-p_{1}\right)\left(s-p_{2}\right) \ldots\left(s-p_{n}\right)}
$$

Alternatively, dividing by the individual poles and zeros, Eq. (A.4-10a) becomes

$$
\frac{\Delta x_{i}(s)}{\Delta u_{j}(s)}=\frac{k_{F}\left(1-\frac{s}{z_{1}}\right)\left(1-\frac{s}{z_{2}}\right) \ldots\left(1-\frac{s}{z_{m}}\right)}{\left(1-\frac{s}{p_{1}}\right)\left(1-\frac{s}{p_{2}}\right) \cdots\left(1-\frac{s}{p_{n}}\right)}
$$

The poles of the transfer function are the roots of the system's characteristic equation, i.e., they are the system's eigenvalues, and they are identical for all transfer functions of the system described by $F$. The zeros depend on $G$ as well as $F$; therefore, they vary from one transfer function to the next. The transfer function gain, $K_{F}$, is the steaciystate value of the transfer function after all transients damp out, assuming that all transients are stable. The transfer function gain, $K_{I}$, is (for most airoraft transfer functions) the initial state rate response to a control step.

The initial value gain, $K_{I}$, is important because it determines the initial slope of a given state variable's step response. This can be seen by app'ying the initial value theorem (Ref. 11) to the state variable's transform, which states that 


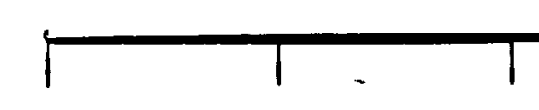

$$
\begin{aligned}
& \lim x(t)=\lim s x(s) \\
& t \rightarrow 0 \quad s \rightarrow \infty
\end{aligned}
$$

if the limit exists. The Laplace transform for the perturbation state, $\Delta x_{i}$, given a unity step input in control, $\Delta u_{j}$, is given as

$$
\Delta x_{i}(s)=\frac{\Delta x_{i}(s)}{\Delta u_{j}(s)}\left(\frac{1}{s}\right)
$$

Since there are more poles than zeros in the transfer functions of interest, the initial value of $\Delta x_{i}(t)$ is zero. physically, this is an indication that the vehicle states do not change instantaneously in response to a control input. The lowest order non-zero state derivative is equal to the excess of poles over zeros. For must aircraft transfer functions of interest, the excess 15 one, and

$$
\Delta \dot{x}_{j}(s)=s \Delta x_{j}(s)=\frac{\Delta x_{i}(s)}{\Delta u_{j}(s)} \frac{s}{s}=\frac{\Delta x_{i}(s)}{\Delta u_{j}(s)}
$$

This leacis to the calculation of the initial value of the state derivative response to a unity step input as

$$
\Delta \dot{x}_{i}(t=0)=K_{I}=\lim _{s \rightarrow \infty} \frac{\Delta x_{i}(s)}{\Delta u_{j}(s)}
$$

Coupled aircraft transfer functions typ ially have seven zeros and eight non-zero poles, so $K_{I}$ and $K_{F}$ are related as follows:

$$
K_{I}=\frac{K_{F}\left(-p_{1}\right)\left(-p_{2}\right) \ldots\left(-F_{8}\right)}{\left(-z_{1}\right)\left(-z_{2}\right) \ldots\left(-z_{7}\right)}
$$


A compaisison of the signs of $K_{I}$ and $K_{F}$, which are related by the signs of the poles and zeros, as indicated by Eq. (A.4-15), is important because these signs give an indication of the expected transient response. If the transfer function is stable and minimum phase, the signs of $K_{I}$ and $K_{F}$ are the same. The resuicing response is sim.lar to the solid line in Fig. 2.5-1. A nonminimum-phase zero causes $K_{I}$ and $K_{F}$ to have opposite signs, and the resulting response resembles one of the dast.ed lines in Fig. 2.5-1. In these responses, the initial response direction is away from the desired final value.

The transfer function has been a fundameutal tool of control system design in the past, and, although linearoptimal control theory serves that purpose in this report (Chapter 4), transfer functions can be valuable for understanding details of the aircraft's dynamics. For example, nonminimum-phase zeros and sign changes in $K_{I}$ can degrade handling qualities (Section 2.2). When poles and zeros are nearly equal, there is a cancelling effect which tends to remove the associated normal mude from the output variable's response to the given input. Conversely, feedback paths between the transfer function's output anc. input have negligible effect on that normal mode. In other words, the transier function provides the information regarding the quality of controllability which was missing in Eq. (A.4-5). These capabilities are put to use in Chapter 2.

\section{A.4.4 Optimal Control Theory}

A regulatoi is a feedback control law which is designed to maintain asymptoticaily stable output of a dynamic system, i.e., it bounds the fluctuations in the output, and it assures that the output goes to zero as time 
increases. An optimal regulator minimizes a cos: ior penalty) functional of the output and control in stabilizing the dynamic system. A linear-optimal regulator minimizes a particular cost function -- the time integral of quadratic functions of the output and controi -- for a linear dynamic system, and it takes the form of Eq. (4.1-1) (Kef. 00). A Iinear-optimal regulator can be designed for an aircraft near, at, or beyond its open-loop departure boundary. This design indicates the control loops which must re closed (either automatically or by the pilot) to prevent departure, providing asymptotic stability and minimizing a quadratic cost functional of the output and control.

The basic design objective for the linear-optimal regulator is to define the feedback contrel law which minimizes a quadratic cost functional, $J$, of the perturbation output vector, $\Delta y(t)$, and the perturbation contiol vector, $\Delta \underline{u}(t)$ :

$$
J=\int_{0}^{\infty}\left[\Delta \underline{y}^{T}(t) Q \Delta \underline{y}(t)+\Delta \underline{u}^{T}(t) R \Delta \underline{u}(t)\right] d t
$$

The control vector contains all available aircraft control displacements -- in this case, throttle setting $\left(\Delta \varepsilon_{\mathrm{T}}\right)$, elevator $\left(\Delta \delta_{h}\right)$, aileron $\left(\Delta \delta_{a}\right)$, and rudder $\left(\Delta \delta_{r}\right)$. The nutput vector represents the measured aircraft variatles and car be formulated as a linear combination of the ajrcraft's perturbation states, $\Delta \underline{x}(t)$, state rates, $\underline{\Delta} \underline{\dot{x}}(t)$, and controls, $\underline{\Delta}(t)$ : the present development uses the simplifying assumption, $\Delta y(t)=\Delta \underline{z}(t)$, where

$$
\Delta \underline{x}^{T}=\left[\begin{array}{llllllll}
\Delta \theta & \Delta u & \Delta z & \Delta w & \Delta v & \Delta r & \Delta p & \Delta \phi
\end{array}\right]
$$

The state-weighting matrix is nonnegative-definite and symmetric, 


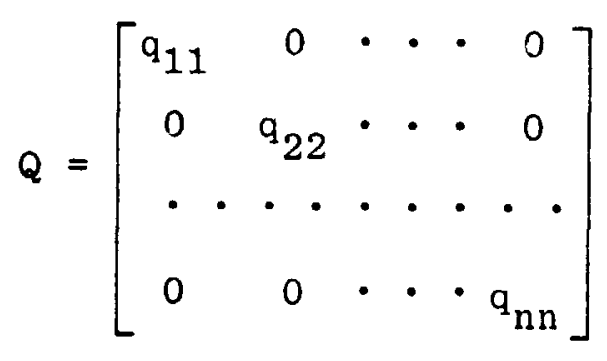

and the control-weighting matrix is positive-definite and symmetric:

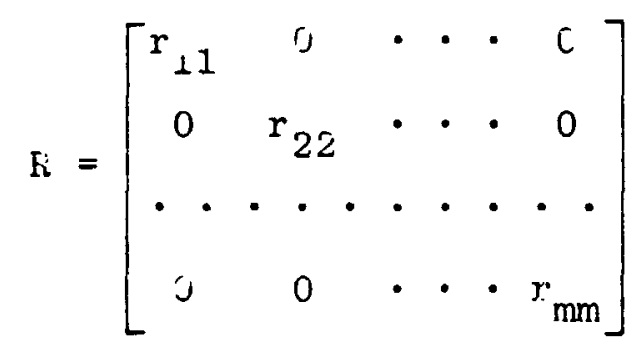

Equatio (A.4-15) can be written as

$$
\begin{aligned}
T=\int_{C}^{\infty} & {\left[q_{11} \Delta x_{1}^{2}(t)+\cdots+q_{n n} \Delta x_{n}^{2}(t)+r_{11} \Delta u_{1}^{2}(t)+\cdots\right.} \\
& \left.+r_{m m} \Delta u_{m}^{2}(t)\right] d t
\end{aligned}
$$

and the cost functional is seen to be a weighted sum of the interiated-square values of the perturbation state and control. In the present case, minimizing the weighted sum of integratadsquare values is equivalent to minimizing the weighted sum of root-mean-square (rms) values of the state and control.

Equation (A.4-20) prorides a means of trading of $f$ the cost of output errors against the cost of control, and it is simply this: choose each we: shting coefficient in $Q$ and $R$ as the inverse of the maximum allowable mean-square value of the weighted variable, i.e., 


$$
\begin{aligned}
& q_{i j}=1 / \Delta x_{i_{\max }}^{2}, \quad i=1 \text { to } n \\
& r_{j j}=1 / \Delta u_{j_{\max }}^{2}, \quad j=1 \text { to } \mathrm{m}
\end{aligned}
$$

This normalizes each term in Eq. (A.4-20), s.. that its contribution to the integrand is unity when the variable equals its maximum value. The elements of $R$ are specified by the control authority which can be assigned to the DFSis. For example, if $10 \mathrm{dog}$ of elevator perturbation can be assigned to departure prevention, the rorresponding elemer $t$ of $R$ is $1 /(10)^{2}=0.01$. If there is prior information regariing allowable state perturbations (as in a tracking tass), the elements of $Q$ are determined similarly.

An alternate approach is to use the elements of $Q$ and $R$ as design parameters which can be varied until desirable transient response or eigenvalues are achieved. In such case, the equivalence of $Q$ elements to allowable mean-square values is not lost, and it is possible to gain insight regarding the correspondence of rms-output errors and classical figures of merit in each particular case.

The minimization of $\mathrm{J}$ must be acconplished subject to the dynamic constraint provided by the linear equation of motion,

$$
\Delta \underline{\dot{x}}(t)=F \underline{x}(t)+G \underline{u}(t)
$$

(It is assumed that $F$ and $G$ form a controllahle nair.) The method of finding the control which minimizes $J$ subject to Eq. (A.4-23) is derived in numerous texts (e.g., Refs. 60 to $62)$. In the special case of quadratic cost and linear system dyramics, the control solution is a linear feedback law (Eq. (4.1-1)). The gain matrix of this control law is 


$$
\mathrm{K}=\mathrm{R}^{-1} \mathrm{G}^{\mathrm{T}} \mathrm{P}
$$

where the symmetric matrix, $P$, is the steady-stite solution of the matrix Riccati equation

$$
\dot{\mathrm{P}}=-\mathrm{PF}-\mathrm{F}^{\mathrm{T} P}+\mathrm{PGR}^{-1} \mathrm{G}^{\mathrm{T}} \mathrm{P}-\mathrm{Q}
$$

In otner words, the DPSAS gain matrix is easily found by two mat. ix multiplications once $P$ is found (Eq. (A.4-24), but the solution for P (Eq. (A.4-25)) appears formidable. There are, however, four recognized methods for steady-state solution of Eq. (A.4-15)), all of which require digital computation (Ref. 60): these are direct integration of Eq. (A.4-25), the Newton-Raphson method, the Kalman-Englar method, and the diagonalization/eigenvalue method. The choice between these methods must be based on grounds of numerical convenience and efficiency.

The Kalman-Englar method (Ref. 73) has been used to generate the results which follow in later sections. In this technique, $\mathrm{P}$ is propagated to steady state using the recursive equation,

$$
P_{k+1}=\left[\theta_{21}+\theta_{22} P_{k}\right]\left[\theta_{11}+\theta_{12} p_{k}\right]^{-1}
$$

The matrices $\theta_{11}, \theta_{12}, \theta_{21}$, and $\theta_{22}$ are the appropriate $(n \times n)$ sub-matrices of

$$
\theta(\Delta t)=e^{-Z \Delta t}
$$

where

$$
Z=\left[\begin{array}{cc}
F & -G R^{-1} G^{T} \\
Q & -F^{T}
\end{array}\right]
$$


and the propagation interval, $\Delta t$, is small compared to the natural periods of the aircraft motion.

The DPSAS design procedure is summarized and shown to be a straightforward technique once the appropriate general-purpose computer routines are programmed:

- Define control authority available to the DPSAS, thus specifying $R$.

- Define allowable state perturbations, thereby specifying $Q$.

- For the aircraft dynamics specified hy the stability and control matrices, $F$ and $G$, compute the feedback gain matrix, K, using Eq. (A.4-28), (A.4-27), $(A .4-26)$, and $(A .4-24)$.

- The control law for the DPSAS is given by Eq. (4.1-1), using the gain matrix calculated in the previour step.

The resulting DPSAS stabilizes the aircraft without using mroe control authority than that specified by $R$ for state perturbations defined by $Q$.

\section{A.4.5 Gain Scr.eduling Procedure}

Means and Siandard Deviations - Two features which suggest that a gain be held constant are its standard deviation and mean value. Certain gains do not exhibit wide variations as the flight conditions change. This can be determined by constructing a table of means and standard deviations for the gains, as illustrated by Table A.4-1. In the table, Gain 6 displays a low standard deviation and a large mean value. This indicates that the gain should probably not be scheduled, i.e., that its mean value can be used at all flight conditions. 
Another feature of each gain is its relative magnitude compared to other gains of a similar class. Gain 4 is small compared to Gain 6 and also exhibits a wide variation in magnitud-; thus it may be desirable to schedule Gain 4, if its variation with flight condition is coherent, or set it to zero. Simulations should be done to fully

TABLE A.4-1.

EXAMPLE OF MEAN-STANDARD DẼVIATION TABLE

\begin{tabular}{c|ccc}
\multicolumn{1}{c}{} & Mean & $\begin{array}{c}\text { Standard } \\
\text { Deviation }\end{array}$ & $\begin{array}{c}\text { S.D. Per Cent } \\
\text { of Mean }\end{array}$ \\
\cline { 2 - 4 } Gain 4 & -.00598 & .0269 & 449.0 \\
Gain 5 & -.1013 & .032 & 31.7 \\
Gain 6 & 2.55 & .046 & 1.82 \\
$\vdots$ & & & \\
etc. & & &
\end{tabular}

determine the zeroed gains' effects on controlling the aircrafi. Gain 5 is a logical candidate for scheduling. The gain magnitude is not negligible, and it displays enough variation to warrant scheduling.

Correlation Between Gains and Flight Variables The aircraft dynamic model varies in a somplex but deterministic way with flight conditions. II the closed-loop response of the aircraft is maintained essentially invariant by automatic control, it is reasonable to assume that the necessary control gains also vary in a complex but deterministic way with flight conditions; hence the gains and flight conditions should be currelated.

The search for gain, flight variable dependencies begins by determining correlation coefficients between the 
gains and all available flight variables. One method of determining the correlation coefficient between a set of gains (dependent variables), $k_{i}$, and a flight variable (independent variable), $m$, is given by the following:

$$
\rho(k, m)=\frac{|\operatorname{cov}[k, m]|}{\sigma(k) \sigma(m)}=\frac{\left|\sum_{i=1}^{I}\left(k_{i}-\bar{k}\right)\left(m_{i}-\bar{m}\right)\right|}{\sqrt{\sum_{i=1}^{I}\left(k_{i}-\bar{k}\right)^{2}} \sqrt{\sum_{i=1}^{I}\left(m_{i}-\bar{m}\right)^{2}}}
$$

In Eq. (A.4-29), I is the number of flight conditions for which the gains are known, $k_{i}$ is the value of the gain observed at flight, oint $i$, and $m_{i}$ is the value of the flight variable at flight point $i$. The variable $k$ is the mean value of the gain, given by

$$
\bar{k}=\frac{I}{T} \sum_{i=1}^{I} k_{i}
$$

and $\bar{m}$ is the nean value of the flight variable. The closer the magnitude of $p$ is to one, the better the correlation between the gain and the flight variable. Independent variables which can be considered for gain scheduling include indicated airspeed (IAS), body-axis velocities $(u, v, w)$ angles of attack and sideslip $(\alpha, \beta)$, angular rates $(p, q, r)$, and control trim positions. These variables can be inverted, squared, and so on, in the search for high correlation. An example of a correlation coefficient taule is shown in Table A.4-2. The circled values are the high correlation coefficients bet.;en gains and independent variable functions. 
TABLE A.4-2

EXAMPLE OF CORRELATION COEFFICIENTS TABLE

\begin{tabular}{l|ccccccccc}
\multicolumn{1}{l}{} & $\alpha$ & $\alpha^{2}$ & $1 / \alpha$ & IAS & IAS2 & $p$ & $q$ & $r$ & ..etc. \\
jain 1 & .838 & .939 & .297 & .939 & .912 & .804 & .018 & .0359 \\
Gain 2 & .879 & .743 & .168 & .745 & .541 & .890 & .028 & .245 \\
Gain 3 & .672 & .786 & .069 & .782 & .904 & .724 & .447 & .339 \\
$\vdots$ & & & & & & & &
\end{tabular}

Curve Fitting - The third step in the gain-scheduling procedure is to construct a smooth relationship between the gains which are to be scheduled and the most highly correlated flight variables. Multiple regression

$$
\hat{k}=b_{0}+b_{1} m_{1}+b_{2} m_{2}+\ldots+b_{n} m_{n}
$$

and polynomial regression,

$$
\hat{k}=b_{0}+b_{1} m_{1}+b_{2} m_{1}^{2}+\ldots+b_{n} m_{i}^{n}
$$

can be used. Equation (A.4-31), the multiple regression, uses $n$ different independent variables, while Eq. (A.4-32), the polynomial regression, uses powers of the highest correlated independent flight variable, $m_{i}$, to estimate the gain, $k$. A method for determining the regression coefficients, $b_{i}$, is shown next.

A multipie regression analysis determines the regression coefficients $b_{0}, b_{1}, \ldots, b_{n}$ in $E q .(A .4-31)$ so that the sum of the squared error between the regression estimate, $\hat{k}$, and the true value of $k$ is minimized. For I flight conditions, the function to be minimized is 


$$
J=\sum_{i=1}^{I}\left(k_{i}-\hat{k}_{i}\right)^{2}
$$

To minimize $J$, set

$$
\frac{\partial J}{\partial b_{0}}=\frac{\partial J}{\partial b_{1}}=\ldots \frac{\partial J}{\partial b_{n}}=0
$$

Then the expression for the regression coefficients becomes

$$
A \underline{b}=\underline{c}
$$

or

$$
\underline{b}=A^{-1} \underline{c}
$$

where $A$ and $\underline{C}$ are defined as in the least-squares formulas of Ref. 63. These are the values of $b_{i}$ which minimize the squared error.

To determine just how good the $b_{i}$ values are, the correlation coefficient for the multiple regression fit can be found, as in Eq. (A.4-?9):

$$
\rho(k, \hat{k})=\frac{\sum_{i=1}^{I}\left(k_{i}-\bar{k}\right)\left(\hat{k}_{i}-\overline{\hat{k}}\right)}{\sqrt{\sum_{i=1}^{I}\left(k_{i}-\bar{k}\right)^{2}} \sqrt{\sum_{i=1}^{I}\left(\hat{k}_{i}-\overline{\hat{k}}\right)^{2}}}
$$

The closer $\rho$ is to one, the better the fit of the multiple regression model. When using a multiple regression, the more independent variables chosen, the higher the value of $\rho$ will be, until $n=I$ and $\rho=1$. 
An alternate way of estimating gain values is to use only one of the flight variables in a polynomial regres-

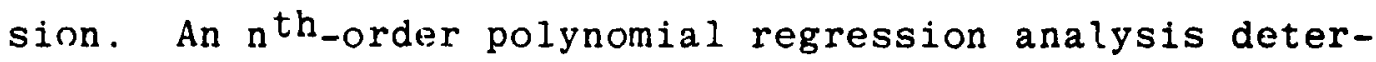
mines the regression coefficients, $b_{0}, b_{1}, \ldots, b_{n}$ in $\mathbf{E q}$. (A.4-32) so that the sum of the squared error between the regression estimate, $\hat{\mathrm{k}}$, of Eq. (A.4-31) and the true value of $k$ is minimum. In this case, there is only one kind of independent varia.le, $m_{i}$, for each gain value, but it is raised to various powers, i.e., $m_{1}=m_{i}, m_{2}=m_{i}^{2}$, until adequate correlation is achieved.

The analysis for the polynomial regression proceecis as in the multiple regression, starting at Eq. (A.4-33). The polynomial regression can be considered a special case of the multiple regression.

\section{A. 4.6 Program ALPHA}

The construction of the complete linear equations of motion, their analysis, and the design of feedback controllers has been programmed in ALPHA--Analysis program for High Angle-of-Attack Stability and Control. Figure 4.4-2 illustrates the structure of this program. Input data consistis of aircraft inertial and aerodynamic characteristics. The aerodynamic data can be entered as conventional stability derivatives, dimensionless derivatives, or full tables of nonlinear force and morient characteristics. 

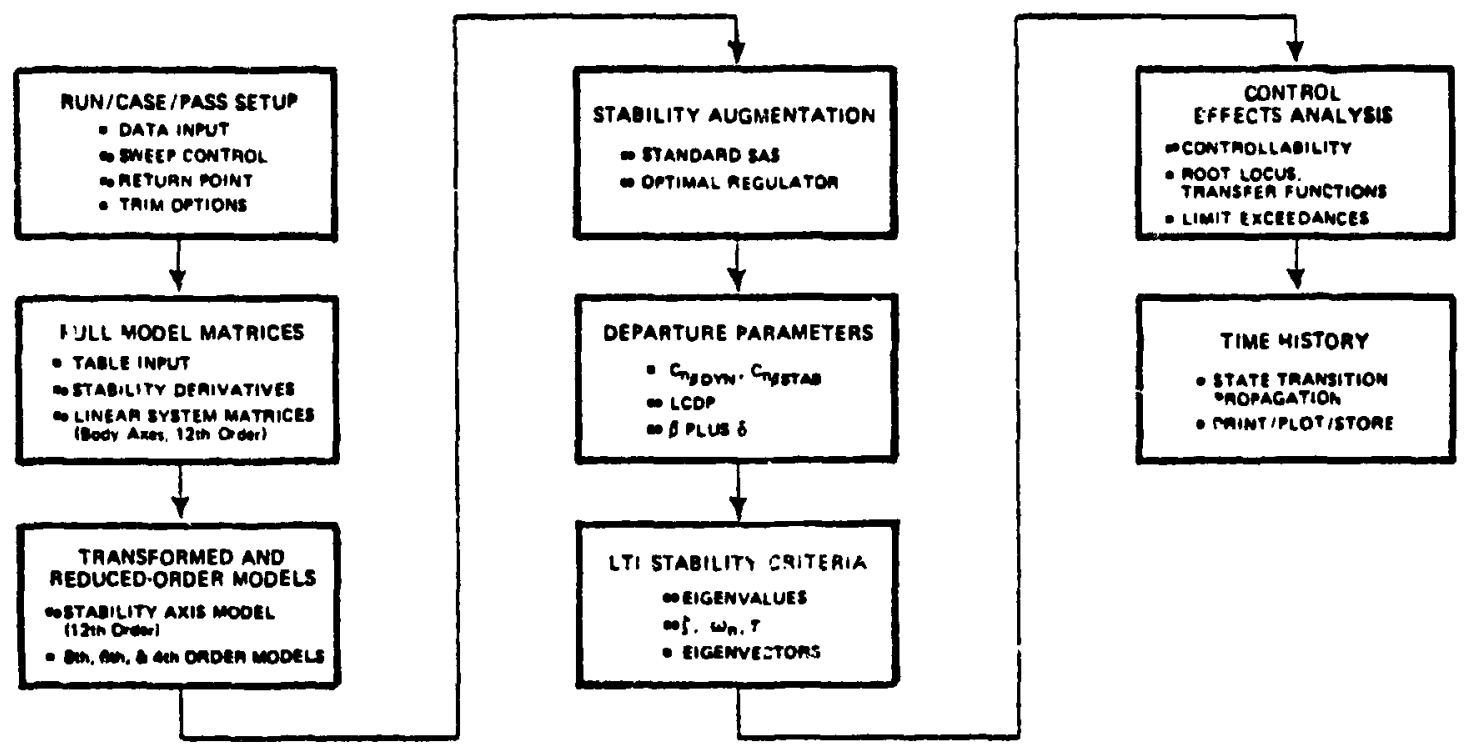

Figure A.4-2 ALPHA - Analysis Program for High Angle-of-Attack Stability and Control.

The program executes a three-step procedure. The first step consists of steady or generalized trim calculation, if desired, and the construction of the complete bodyaxis system dynamics and control input matrices. During the second step, the linear system is modified, if required, to include any axis transformation, order reduction, or fixed stability augmentation loop closure. The final step consists of the analysis of the resulting system. Eigenvalues, eigenvectors, transfer functions, linear-optiral stability augmentation systems, and time histories can be calculated and plotted. 
The executive structure of ALPHA includes logic to modify the dynamic model on succeeding passes through the program and to vary the analysis type as ceitain parameters are varied over a given range of interest. Program ALPHA provides an efficient tool for the thorough analysis of alrcraft high angle-of-attack stability and control. 


\section{APPENDIX B}

\section{AIRCRAFT AERODYNAMIC MODEL}

The reference aircraft is a small, supersonic fighter type designed for air superiorty missions. Hass, dimensional, and Inertial characteristics are listed in Table B-1. The aerodynamic data set is a composite of sub-scale wind tinnel measurements for two configurations of the reference aircraft; bence, the numerical results presented here do not represent a specific aircraft in detall.

TABLE B-1

CHARACTERISTICS OF THE REFERENCE AIRCRAFT

\begin{tabular}{|c|c|}
\hline Mass, kg & 6,124 \\
\hline$I_{x}, \quad k g-m^{2}$ & 7,186 \\
\hline$I_{y}, k g-m^{2}$ & 57,350 \\
\hline$I_{z}, k g-m^{2}$ & 63,450 \\
\hline$I_{x z}, \quad k g-m^{2}$ & -149 \\
\hline Reference Area, $\mathrm{m}^{2}$ & 17.30 \\
\hline Mean Aerodyramic Chord, $m(\bar{c})$ & 2.46 \\
\hline Wing Span, $m(b)$ & 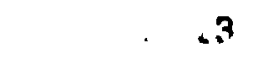 \\
\hline Lengtb, m & .73 \\
\hline Reference Center of Gravity (c.g.) & $0.25 \bar{c}$ \\
\hline Flight c.g. & $0.17 \bar{c}$ \\
\hline
\end{tabular}


The control variables are elevator (or horicontal tall, $\left.\delta_{h}\right)$, leading/trailing edge flaps $\left(\delta_{f}\right)$, ailerons $\left(\delta_{a}\right)$, rudder $\left(\delta_{\mathrm{r}}\right)$, speed brake $\left(\delta_{\mathrm{SB}}\right)$, and irust secting $\left(\delta_{\mathrm{T}}\right)$. The ranges of these variables are listed in Table $\mathrm{B}-2$.

TABLE B-2

CONTROL VARIABLE RANGES

$\begin{array}{lr}\delta_{\mathrm{h}} & -20 \text { to }+5 \mathrm{deg} \\ \delta_{\mathrm{f}} & 0 \text { to } 100 \text { percent } \\ \delta_{\mathrm{a}} & -60 \text { to }+60 \mathrm{deg} \\ \delta_{\mathrm{r}} & -30 \text { to }+30 \mathrm{deg} \\ \delta_{\mathrm{SB}} & 0 \text { to } 45 \mathrm{deg} \\ \delta_{\mathrm{T}} & 0 \text { to } 100 \text { percent }\end{array}$

The aerodynamics of the aircraft are represented by 45 coe: icients which are functions of argle of attack, sideslip angle, elevator deflection, and flap setting. Using simpliried conventional notation, the 6 tctal coefficients are described as follows:

$$
\begin{aligned}
\mathrm{C}_{\mathrm{X}_{\mathrm{T}}}= & \mathrm{C}_{\mathrm{X}}(\alpha, \beta)+\Delta \mathrm{C}_{\mathrm{X}}\left(\alpha, \beta, \delta_{\mathrm{f}}\right)+\Delta \mathrm{C}_{\mathrm{X}}\left(\alpha, \delta_{\mathrm{SB}}\right) \cdot \Delta \mathrm{C}_{\mathrm{X}}\left(\alpha, \delta_{\mathrm{f}}, \delta_{\mathrm{h}}\right) \\
& +\frac{\bar{c} \mathrm{~g}}{2 \mathrm{~V}} \mathrm{C}_{\mathrm{X}_{\mathrm{q}}(\alpha)} \\
\mathrm{C}_{\mathrm{Y}_{\mathrm{T}}}= & \mathrm{C}_{\mathrm{Y}_{0}}\left(\alpha, \delta_{\mathrm{f}}\right)+\Delta \mathrm{C}_{\mathrm{Y}}(\alpha, \beta)+\Delta \mathrm{C}_{\mathrm{Y}}\left(\alpha, \beta, \delta_{\mathrm{f}}\right)+\Delta \mathrm{C}_{\mathrm{Y}}\left(\alpha, \delta_{\mathrm{f}}, \delta_{\mathrm{a}}\right) \\
& +\Delta \mathrm{C}_{\mathrm{Y}}\left(\alpha, \delta_{\mathrm{Y}}\right)+\frac{b}{2 \mathrm{~V}}\left[\mathrm{C}_{\mathrm{Y}_{\mathrm{Y}}}(\alpha) \mathrm{r}+\mathrm{C}_{\mathrm{Y}_{\mathrm{p}}}(\alpha) \mathrm{p}+\mathrm{C}_{\mathrm{Y} \dot{B}}(\alpha) \dot{B}\right]
\end{aligned}
$$




$$
\begin{aligned}
& C_{Z_{T}}=C_{Z}(\alpha, \beta)+\Delta C_{Z}\left(\alpha, \beta, \delta_{f},+\Delta C_{Z}\left(\alpha, \delta_{S B}\right)+\Delta C_{Z}\left(\alpha, \delta_{\perp} \delta_{h}\right)\right. \\
& +\frac{\bar{c} g}{2 V} c_{Z_{q}}(\alpha) \\
& c_{1_{T}}=\tau_{1_{0}}\left(\alpha, \delta_{f}\right)+C_{1}(\alpha, \beta)+\Delta C_{1}\left(\alpha, \beta, \delta_{f}\right)+\Delta C_{1}\left(\alpha, \delta_{f}, \delta_{l}\right) \\
& +\Delta I_{1}\left(\alpha, \delta_{r}\right)+\frac{b}{2 V}\left[C_{1_{r}}(\alpha) r+C_{1_{p}}(\alpha) p+C_{1_{\dot{\beta}}}(\alpha) \dot{\beta}\right] \\
& C_{m_{T}}=C_{n_{0}}+C_{m}(\alpha, \beta)+C_{Z_{m}}(\Delta \bar{x})+\Delta C_{m}\left(\alpha, \beta, \delta_{f}\right)+1 C_{m}\left(u, \delta_{f}, \delta_{h}\right) \\
& +\Delta C_{m}\left(\alpha, \delta \delta_{S B}\right)+\frac{\bar{c} g}{2 v} C_{m_{q}}(\alpha) \\
& c_{n_{T}}=c_{n_{0}}\left(\alpha, \delta_{f}\right)+c_{n}(\alpha, \beta)+\Delta c_{Y_{T}}(\Delta \bar{x}) \frac{\bar{c}}{b}+\Delta c_{n}\left(\alpha, \beta, \delta_{f}\right) \\
& +\Delta C_{n}\left(\alpha, \delta_{f}, \delta_{a}\right)+\Delta C_{n}\left(\alpha, \delta_{r}\right) \\
& +\frac{b}{2 V}\left[C_{n_{r}}(\alpha) r+C_{n_{p}}(\alpha) p+C_{n_{\dot{\beta}}}(\alpha) \dot{B}\right]
\end{aligned}
$$

The first step in finding the individual terms of the perturbat: $:$ forces and moments, Eqs. (A.3-13) to (A.3-16), is to evaluate the derivatives of the force and moment coefficjents with respect to the nondimensirnal states at the nominal filight condition. This results in nondimensional stability derivatives, such as the followinf: 


$$
\begin{aligned}
& c_{X_{u}}=\frac{\partial C_{X_{T}}}{\partial\left(u / v_{0}\right)}=\left[-\frac{\bar{c}_{q_{0}}}{2 v_{0}} c_{X_{q}}\left(\alpha_{0}\right)\right] \frac{\partial\left(v / v_{0}\right)}{\partial\left(u / v_{0}\right)} \\
& +\left[\frac{\partial C_{X}\left(\alpha_{0}, \beta_{0}\right)}{\partial \beta}+\frac{\partial \Delta C_{X}\left(\alpha_{0}, \beta_{0}, \delta_{f_{0}}\right)}{\partial \beta}\right] \frac{\partial \beta}{\partial\left(u / V_{0}\right)} \\
& +\left[\frac{\partial C_{X}\left(\alpha_{0}, \beta_{0}\right)}{\partial \alpha}+\frac{\partial \Delta C_{X}\left(\alpha_{0}, \beta_{0}, \delta_{f_{0}}\right)}{\partial \alpha}\right. \\
& +\frac{\partial \Delta \mathrm{C}_{\mathrm{X}}\left(\alpha_{\sigma^{\prime}}{ }_{\mathrm{SB}}\right)}{\partial \alpha}+\frac{\partial \Delta \mathrm{C}_{\mathrm{X}}\left(\alpha_{0}, \delta_{f_{n}}, \delta_{f_{n}}\right)}{\partial \alpha} \\
& \left.+\frac{\bar{c} q_{0}}{2 V_{0}} \frac{\partial C_{m_{q}}\left(\alpha_{0}\right)}{\partial \alpha}\right] \frac{\partial \alpha}{\partial\left(u / v_{0}\right)} \\
& C_{X_{q}}=\frac{\partial C_{X_{T}}}{\partial\left(\frac{q \bar{c}}{2 V}\right)}=C_{X_{q}}\left(\alpha_{0}\right)
\end{aligned}
$$

Many of these derivatives contain the partial derivatives of the nondimensional wind-axis translational velocities $\left(\mathrm{V} / \mathrm{V}_{0}, \beta, \alpha\right)$ with respect to the nondimensional body-axis translational velocities $\left(u / V_{0}, v / V_{0}, w / V_{0}\right)$. This matrix of derivatives, evaluated at the sominal flight condition, is

$$
\begin{gathered}
\frac{\partial\left(v / V_{0}, \beta, \alpha\right)^{T}}{\partial\left(i \alpha / v_{0}, v / v_{0}, w / V_{0}\right)^{T}} \mid=\left[\begin{array}{cccc}
\cos \alpha_{0} \cos \beta_{0} & \sin \beta_{0} & \sin \alpha_{0} \cos \beta_{0} \\
-\cos \alpha_{0} \sin \beta_{0} & \cos \beta_{0} & -\sin \alpha_{0} \sin \beta_{0} \\
-\sin \alpha_{0} / \cos \beta_{0} & 0 & \cos \alpha_{0} / \cos \beta_{0}
\end{array}\right] \\
v_{0}, \alpha_{0}, \beta_{0}
\end{gathered}
$$


The dimensional stability derivatives are formed by taking the derivatives of the dimensional aerodynamic forces and moments with respect to the dimensional state variables. These dimensional derivatives contain the nondimensional derivatives; $\frac{\partial F_{X}}{\partial u}$ and $\frac{\partial F_{X}}{\partial q}$ are examples of these derivatives:

$$
\begin{aligned}
& \frac{\partial F_{X}}{\partial u}=\frac{\partial}{\partial u}\left[\frac{1}{2} \rho V^{2} S C_{X T}\right]
\end{aligned}
$$

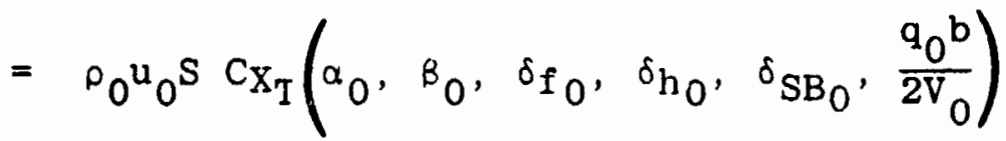

$$
\begin{aligned}
& +\frac{1}{2} \rho_{0} V_{0} S C_{u} \\
& \frac{\partial F_{X}}{\partial q}=\frac{\partial}{\partial q}\left[\frac{1}{2} \rho V^{2} S C_{T}\right] \\
& =\frac{1}{2} \rho_{0} v_{0}^{2} s C_{X_{q}} \frac{\bar{c}}{2 v_{0}}
\end{aligned}
$$

The complete dimensional stability derivative matrices are

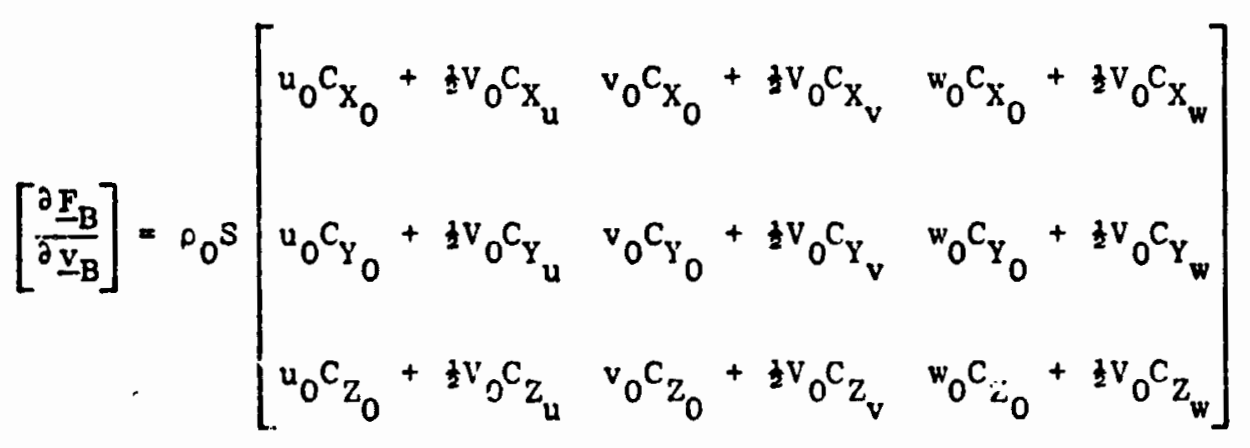


$\left[\frac{\partial \underline{F}_{B}}{\partial \underline{\omega}_{B}^{I}}\right]=\frac{\rho_{O}}{4} \gamma_{O} s\left[\begin{array}{ccc}b c_{X_{p}} & \bar{c} c_{X_{q}} & b c_{X_{r}} \\ b c_{Y_{p}} & \bar{c} c_{Y_{q}} & b c_{Y_{r}} \\ b c_{Z_{p}} & \bar{c} c_{Z_{q}} & b c_{Z_{r}}\end{array}\right]$

$\left[\frac{\partial \underline{F}_{B}}{\partial \underline{u}}\right]=q_{\infty 0} s\left[\begin{array}{llll}c_{X_{\delta T}} & c_{X_{\delta h}} & c_{X_{\delta a}} & c_{X_{\delta r}} \\ c_{Y_{\delta T}} & c_{Y_{\delta h}} & c_{Y_{\delta a}} & c_{Y_{\delta r}} \\ c_{Z_{\delta T}} & c_{Z_{\delta h}} & c_{Z_{\delta a}} & c_{Z_{\delta r}}\end{array}\right]$

$\left[\frac{\partial F_{B}}{\partial \dot{\underline{v}}_{B}}\right]=\frac{\rho}{4} S\left[\begin{array}{ccc}\bar{c} c_{X_{\dot{u}}} & b c_{X_{\dot{v}}} & \bar{c} c_{X_{\dot{w}}} \\ \bar{c} c_{Y_{\dot{u}}} & b c_{Y_{\dot{v}}} & \bar{c} c_{Y_{\dot{w}}} \\ \bar{c} c_{Z_{\dot{u}}} & b C_{Z_{\dot{v}}} & \bar{c} c_{Z_{\dot{w}}}\end{array}\right]$

$\left[\frac{\partial \underline{F}_{B}}{\partial \underline{\omega}_{B}^{I}}\right]=\frac{{ }_{0}}{8} S\left[\begin{array}{ccc}b^{2} c_{X_{\dot{p}}} & \bar{c}^{2} c_{X_{\dot{q}}} & b^{2} c_{X_{\dot{r}}} \\ b^{2} C_{Y_{\dot{p}}} & \bar{c}^{2} c_{Y_{\dot{q}}} & b^{2} C_{Y_{\dot{r}}} \\ b^{2} c_{Z_{\dot{p}}} & \bar{c}^{2} c_{Z_{\dot{q}}} & b^{2} c_{Y \dot{r}}\end{array}\right]$ 


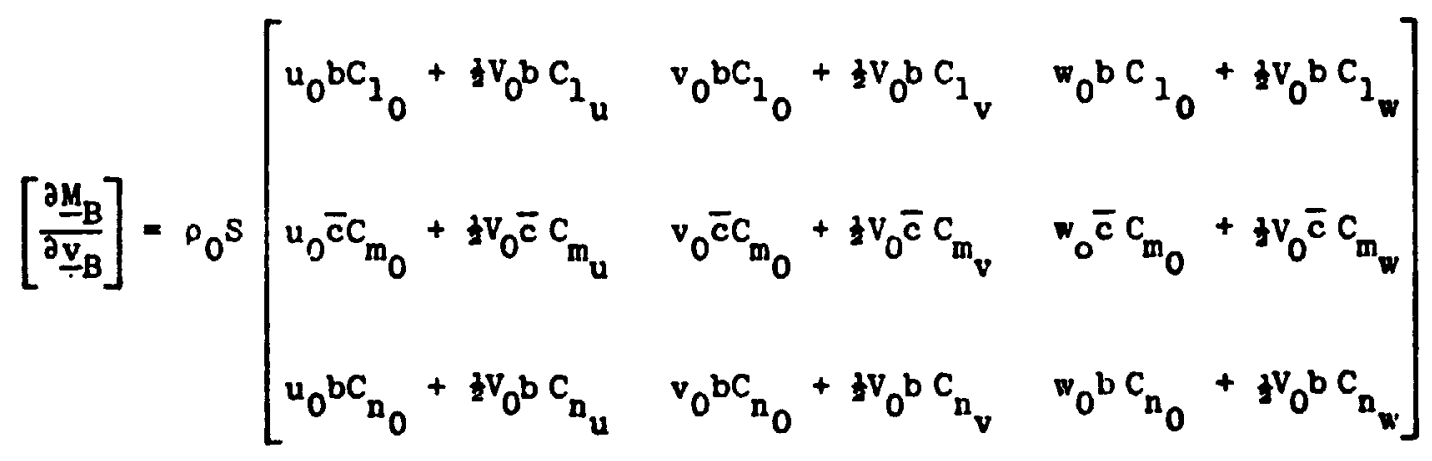

$$
\begin{aligned}
& {\left[\begin{array}{c}
\frac{\partial \underline{\mu}_{B}}{\partial \underline{\omega}_{B}}
\end{array}\right]=\frac{\rho_{0}}{4} v_{0} s\left[\begin{array}{ccc}
b^{2} c_{1_{p}} & b \bar{c} c_{1_{q}} & b^{2} c_{1_{r}} \\
b \bar{c} c_{m_{p}} & \bar{c}^{2} c_{m_{q}} & b \bar{c} c_{m_{r}} \\
b^{2} c_{n_{p}} & b \bar{c} c_{n_{q}} & b^{2} c_{n_{r}}
\end{array}\right]} \\
& {\left[\frac{\delta \underline{\underline{M}}}{\delta \underline{u}}\right]=q_{\infty 0} s\left[\begin{array}{llll}
b c_{1_{\delta T}} & b c_{1_{\delta h}} & b c_{1_{\delta a}} & b c_{1_{\delta r}} \\
\bar{c} c_{m_{\delta T}} & \bar{c} c_{m_{\delta h}} & \bar{c} c_{m_{\delta \zeta}} & \bar{c}_{m_{\delta r}} \\
b c_{n_{\delta T}} & b c_{n_{\delta h}} & b c_{n_{\delta a}} & b c_{n_{\delta r}}
\end{array}\right]} \\
& {\left[\frac{\partial \underline{M}_{B}}{\partial \dot{v}_{B}}\right]=\frac{\rho_{0}}{4} s\left[\begin{array}{ccc}
b \bar{c} c_{1_{\dot{u}}} & b^{2} c_{1_{\dot{v}}} & b \bar{c} c_{1_{\dot{w}}} \\
\bar{c}^{2} c_{m_{\dot{u}}} & b \bar{c} c_{m_{\dot{v}}} & \bar{c}^{2} c_{m_{\dot{w}}} \\
b \bar{c} c_{n_{\dot{u}}} & b^{2} c_{n_{\dot{v}}} & b \bar{c} c_{n_{w}}
\end{array}\right]}
\end{aligned}
$$



REFERENCES

1. Lanchester, F.W., Aerodonetics, A. Constable, London, 1908.

2. Bairstow, L., Jones, B.M. and Thompson, B.A., "Investigation into the Stability of an Airplane," ARC R\&M 77, London, 1913.

3. Phillips, W.H., "Eifect of Steady Rolling on Long1tudinal and Directional Stability," NACA TN 1627, Washington, June 1948 .

4. Abzug, M.J., "Effects of Certain Steady Motions on Sma11-Disturbance Airplane Dynamics," Journal of the Aeronautical Sciences, Vol. 21, No. 11, November 1954, pp. 749-762.

5. Moul, M.T. and Paulson, J.W., "Dynamic Lateral Behavior of High Performance Aircraft," NASA RM L58E16, Washington, August 1958.

6. Porter, D.F. and Loomis, J.P., "Examination of an Aerodynamic Coupling Phenomenon," Journal of Aircraft, Vol. 2, No. 6, November-December 1965, pp.553-556.

7. Hamel, P., "A System Analysis View of Aerodynamic Coupling," Journal of Aircraft, Vol. 7, No. 6, NovemberDecember 1970, pp. 567-569.

8. McConnell, C.W., "Application of the Lateral and Longitudinal (Coupled) Equations of Motion to an InertiallySlender Aircraft in Unsymmetric, Rectilinear Flight," M.S. Thesis, Air Force Institute of Technology, December 1973.

9. Johnston, D.E. and Hogge, J.R., "The Effect of Nonsymmetric Flight on Aircraft High Angle of Attack Handling Qua?ities and Departure Characteristics," AIAA Paper No. 74-792, New York, August 1974.

10. Stengel, R.F., "Effect of Combined Roll Rate and Sideslip Angle on Aircraft Flight Stability," Journal of Alrcraft, Vol. 12, No. 8, August 1975, pp. 683-685. 


\section{REFERENCES (Continued)}

11. Greer, H.D., "Summary of Directional Divergence Characteristics of Several High-Performance Aircraft Configurations," NASA TN D-6993, Washington, November 1972.

12. Weissman, R., "Preliminary Criteria for Predicting Departure Characteristics/Spin Susceptibility of Fighter-Type Aircraft," Journal of Aircraft, Vol. 10, No. 4, April 1973, pp. 214-218.

13. Pelikan, R.J., "Evaluation of Aircraft Departure Divergence Criteria with a Six-Degree-of-Firedom Digital Simulation Program," AIAA Paper No. 74-68, New York, January 1974.

14. Weissman, R., "Status of Design Criteria for Predicting Departure Characteristics and Spin Susceptibility," AIAA Paper No. 74-791, August 1974.

15. Ross, A.J., "Investigation of Nonlinear Motion Experienced on a Slender-Wing Research Aircraft," Journal of Aircraft, Vol. 9, No. 9, September 1972, pp.625-631.

16. Haddad, E.K., "Study of Stability of Large Maneuvers of Airplanes," NASA CR-2447, Washington, August 1974.

17. Wykes, J.H., "An Analytical Study of the Dynamics of Spinning Aircraft," WADC TR 58-381, Part III, WrightPatterson AFB, February 1960.

18. Scher, S.H., "Post-Stall Gyrations and Their Study on a Digital Computer," AGARD Report 359, Paris, April 1961.

19. Anglin, E.L. and Scher, S.H., "Analytical Study of Aircraft-Developed Spins and Determination of Moments Required for Satisfactory Spin Recovery," NASA TN D-2181, Washington, February 1964.

20. Grafton, S.B., "A Study to Determine Effects of Applying Thrust on Recovery from Incipient and Developed Spins for Four Airplane Configurations," NASA TN D-3416, Washington, June 1966.

21. Powers, B.G., "A Parametric Study of Factors Influencing the Deep-Stall Pitch-Up Characteristics of T-Tail Transport Aircraft," NASA TN D-3370, Washington, August 1966. 


\section{REFERENCES (Continued)}

22. Bihrle,W., Jr., "Influence of the Static and Dynamic Aerodynamic Characteristics on the Spinning Motion of Aircraft," Journal of Aircraft, Vol. 8, No. 10, October 1971, pp. 764-768.

23. Moore, F.L., Anglin, E.L., Adams, M.S., Deal, P.O. and Person, L.H., Jr., "Utilization of a Fixed-Base Simulator to Study the Stall and Spin Characteristics of Fighter Airplanes," NASA TN D-6+17, Washington, March 1971.

24. Champoux, R.L., "The Numerical Solution Analysis of Airplane Spin Equations Modeled in a Fixed Coordinate System," M.S. Thesis, Naval Postgraduate School, December 1972 .

25. Bihrle, W., Jr., "Effects of Several Factors on Theoretical Predictions of Airplane Spin-Characteristics, NASA CR-132521, Langley Research Center, August 1974.

26. Willen, T.B. and Johnson, K., "Anticipated Spin Susceptibility Characteristics of the A-10 Aircraft," AIAA Paper No. 75-33, New York, January 1975.

27. Eney, J.A. and Chambers, J.R., "Piloted Simulation of Spin," ASD Stall/Post-Stall/Spin Symposium, WrightPatterson AFB, December 1971.

28. Bowman, J.S., Jr., "Airplane Spinning," Astronautics and Aeronautics, Vol. 4, No. 3, March 1966, pp.64-67.

29. Adams, W.M., Jr., "Analytic Prediction of Airplane Equilibrium Spin Characteristics," NASA TN D-6926. Washington, November 1972.

30. Johnson, A.E., "A Literature Survey of the Problem of Aircraft Spins," M.S. Thesis, Naval Postgraduate School, September 1971.

31. McRuer, D.T., and Johnston, D.E., "Flight Control Systems Properties and Problems, Volume I," NASA CR-2500, Washington, February 1975.

32. Hawkins, M.L., "An Investigation of the Departure Modes of a F-4D Aircraft from a Steady Sideslip Flight Condition," M.S. Thesis, Air Force Institute of Technology, December 1974. 


\section{REFERENCES (Continued)}

33. Rudolph, R.J., "Stability Analysis of an F-4C Aircraft in Steady Level Turning Flight," M.S. Thesis, Air Force Institute of Technology, December 1974.

34. Chambers, J.R. and Anglin, E.L., "Analysis of LateralDirectional Stability Characteristics of a Twin-Jet Fighter Airplane at High Angles of Attack," NASA TN D-5361, Washington, August 1969.

35. Chambers, J.R., Anglin, E.L. and Bowman, J.S., Jr. , "Effects of a Pointed Nose on Spin Characteristics of a Fighter Airplane Model Including Correlation with Theoretical Calculations," NASA TN D-5921, Washington, September 1970 .

36. Chambers, J.R. and Bowman, J.S., Jr., "Recent Experience with Techniques for Prediction of Spin Characteristics of Fighter Aircraft," Journal of Aircraft, Vol. 8, No. 7, July 1971, pp. 548-553.

37. Coe, P.L., Jr., Chambers, J.R., and Letko, W.,

"Asymmetric Lateral-Directicnal Characteristics of Pointed Bodies of Revolution at High Angles of Attack," NASA TN D-7095, Washington, November 1972.

38. Ray, E.J., Mckinney, L.W., and Carmichael, J.G., "Maneuver and Buffet Characteristics of Fighter Aircraft," NASA TN D-7131, Washington, July 1973.

39. Grafton, S.B., Chambers, J.R., and Coe, P.L., Jr., "Wind-Tunnel Free- Flight Investigation of a Model of a Spin-Resistant Fighter Configuration," NASA TN D-7716, Washington, June 1974.

40. Tobak, M. and Schiff, L.B., "Nonlinear Aerodynamics of Aircraft in High Angle-of-Attack Maneuvers." AIAA Paper No. 74-85, New York, January 1974.

41. Coe, P.L., Jr. and Newsoni, W.A., Jr., "Wind-Tunnel Investigation to Determine the Low-Speed Yawing Stability Derivatives of a Twin-Jet Fighter Model at High Angles of Attack," NASA TN D-7721, Washington, August 1974 .

42. Hwang, C. and Pi, W.S., "Transonic Buffet Behavior of Northrop F-5A Aircraft," AGARD Report 624, Neuillysur-Seine, September 1974. 


\section{REFERENCES (Continued)}

43. Taylor, C.R., ed., "Aircraft Stalling and Buffeting," AGARD Lecture Series No. 74, Neuilly-sur-Seine, February 1975.

44. Kao, H.C., "Side Forces on Unyawed Slender Inclined Aerodynamic Bodies," Journal of Aircraft, Vol. 12, No. 3, March 1975, pp. 142-150.

45. Tobak, M. and Schiff, L.B., "Generalized Formulation of Nonlinear Pitch-Yaw-Roll Coupling: Part I-Nonaxisymmetric Bodies and Part II-Nonlinear ConingRate Dependence," AIAA Journal, Vol. 13, No. 2, March 1975, pp. 323-332.

46. Nguyen, L.T., "Evaluation of Importance of Lateral Acceleration Derivatives in Extraction of LateralDirectional Derivatives at High Angles of Attack," NASA TN D-7739, Washington, October 1974 .

47. Neihouse, A.I., Klinar, W.J. and Scher, S.H., "Status of Spin Research for Recent Airplane Designs," NASA TR R-57, Washington, 1960.

48. Fuchs, R. and Schmidt, W., "The Dangerous Flat Spin and the Factors Affecting It," NACA TM 629, Washington, 1931.

49. Young, J.W., "Optimal and Suboptimal Control Technique for Aircraft Spin Recovery," NASA TN D-7714, Washington, October 1974 .

50. Gilbert, W.P. and Libbey, C.E., "Investigation of an Automatic Spin-Preventing System for Fighter Airplanes," NASA TN D-6670, Washington, March 1972.

51. Chen, R.T., Newell, F.D., and Schelhorn, A.E., "Development and Evaluation of an Automatic Departure Prevention System and Stall Inhibitor for Fighter Aircraft," AFFDL TR-73-29, Wright-Patterson AFB, April 1973.

52. Anderson, C.A, and Walker, J.E., III, "A Stall Inhibitcr for the F-111," NAECON'72 Record, May 1972.

53. Lamars, J.P., "Design for Departure Prevention in the YF-16," AIAA Paper No. 74-794, New York, August 1974. 


\section{REFERENCES (Continued)}

54. McDonald, E.H., "F-15 Eagle Flight Control System" in Advances in Control Systems, AGARD CP-137, Neuil1y-sur-Seine, May 1974.

55. Lindahl, J.H., "Application of the Automatic Rudder Interconnect (ARI) Function in the F-14 Alrcraft," ONR Symposium on Optimal Control, Monterey, July 1975.

56. Nguyen, L.T., Anglin, E.L., and Gilbert, W.P., "Recent Research Related to Prediction of Stail/Spin Characteristics of Fighter Aircraft," Proceedings of the AIAA 3rd Atmospheric Flight Mechanics Conference, Arlington, Texas, June 1976, pp. 79-91.

57. Price, C.F. and Koenigsberg, W.D., "Adaptive Control and Guidance for Tactical Missiles," The Analytic Sciences Corp., TR-170-1, June 1970 .

58. Stengel, R.F., Broussard, J.R. and Berry, P.W., "The Design of Digital-Adaptive Controllers for VTOL Alrcraft," The Analytic Sciences Corp., TR-640-1, NASA CR-144912, March 1976.

59. Kaufman, H., Alag, G., Berry, P. and Kotob, S., "Digital Adaptive Flight Controller Development," NASA CR-2466, Washington, December 1974.

60. Kwakernaak, H. and Sivan, R., Linear Optimal Control Systems, Wiley-Interscience, New York, 1972.

61. Athans, M. and Falb, P.L., Optimal Control, McGrawH111, New York, 1966.

62. Bryson, A.E., Jr, and Ho, Y.C., Applied Optimal Control, Ginn-Blaisdel1, Waltham, 1969.

63. Gelb, A. ed., Applied Optimal Estimation, M.I.T. Press, Cambridge, 1974.

64. Perkins, C.F. and Hage, R.E., Airplane Performance Stability and Control, J. Wiley and Sons, New York. 1949 .

65. Etkin, B., Dynamics of Atmospheric Flight, J. Wiley and Sons, New York, 1972.

66. Seckel, E., Stability and Control of Airplanes and Helicopters, Academic Press, New York, 1964. 


\section{REFERENCES (Continued)}

67. McRuer, D., Ashkenas, I and Graham, D., Aircraft Dynamics and Automatic Control, Princeton University Press, Princeton, 1973.

68. Anglin, E.L., "Static Force Tests of a Model of a Twin-Jet Fighter Airplane for Angles of Attack from $-10^{\circ}$ to $110^{\circ}$ and Sideslip Angles from -400 to $40^{\circ}$," NASA TN D-6425, Nashington, August 1971,

69. Grafton, S.B. and Libbey, C.E., "Dynamic Stability Derivatives of a Twin-Jet Fighter Model for Angles of Attack from $-10^{\circ}$ to $110^{\circ}$," NASA TN D-6091, Washington, September 1970.

70. Jacob, H.G., "An Engineering Optimization Method with Application to STOL-Aircraft Approach and Landing Trajectories," NSAS TN D-6978, Washington, September 1972.

71. DeRusso, P.M., Roy, R.J. and Close, C.M., State Variables for Engineers, J. Wiley and Sons, New York, 1967.

72. Patel, R.V., "On the Computation of Numerators of Transfer Functions of Linear Systems," IEEE Transac= tions on Automatic Control, Vol. AC-18, No. 4, August 1973.

WU.S. GOVERMENT PRINTING OFFICE, 1977 - 735-004/2 